\title{
Efficiency In \\ Inguinal Hernia Repair
}

Coen V. van Hessen 



\section{Efficiency In \\ Inguinal Hernia Repair}

Coen V. van Hessen 


\section{Efficiency in inguinal hernia repair}

Phd thesis, Utrecht University, The Netherlands

The studies described in this thesis were financially supported by the Cornelis Visser Foundation.

Financial support for the printing of this thesis was provided by ChipSoft, Diakademie, Dutch Hernia Society, Stichting Wetenschap Chirurgie Diakonessenhuis, UMC Utrecht Cancer Center and Soave Records.

\section{Lay-Out}

Tjeerd Corjanus

\section{Printing}

Proefschriftmaken.nl

(C) Coen V. van Hessen, Utrecht, 2020

All rights reserved. No part of this thesis may be reproduced or transmitted in any form or by any means without prior written permission from the author, The copyrigth of the papers that have been published or have been accepted for publication has been transferred tot he respective journals.

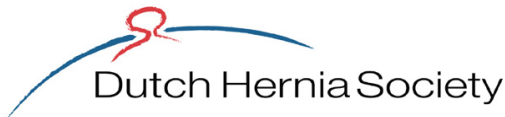




\title{
Efficiency in inguinal hernia repair
}

Doelmatige liesbreukzorg (met een samenvatting in het Nederlands)

\begin{abstract}
PROEFSCHRIFT
ter verkrijging van de graad van doctor aan de Universiteit Utrecht, op gezag van de rector magnificus prof. dr. H.R.B.M. Kummeling, ingevolge het besluit van het college voor promoties

in het openbaar te verdedigen

op donderdag 26 november 2020 des middags te 16:15
\end{abstract}

door

Coenraad Victor van Hessen

geboren op 1 november 1991

te Utrecht 
Copromotoren:

Dr. J.P.J. Burgmans

Dr. E.J.M.M. Verleisdonk 


\section{TABLE OF CONTENTS}

CHAPTER 1

CHAPTER 2

CHAPTER 3

CHAPTER 4

CHAPTER 5

CHAPTER 6

CHAPTER 7

CHAPTER 8

CHAPTER 9

CHAPTER 10

CHAPTER 11

APPENDICES

practice

Submitted

Incidental inguinal hernia in men

Submitted

What can be learned from reoperated groins after endoscopic totally extraperitoneal (TEP) inguinal hernia repair? An 11-year analysis.

Hernia 2018

Recurrence after totally extraperitoneal (TEP) inguinal hernia repair; the role of physical examination and ultrasound Hernia 2019

Is young age a risk factor for chronic postoperative inguinal pain after endoscopic totally extraperitoneal (TEP) repair? Hernia 2019

Antibiotic prophylaxis for prevention of postoperative wound infection in adults undergoing open elective inguinal or femoral hernia repair (Review)

Cochrane 2020 (in press)

Summarizing discussion, conclusions and future perspectives NTVG 2018 


\section{INTRODUCTION}

An inguinal hernia is one of the most common surgical diagnose and its repair accounts for an important part of health care expenditure. Annually, over 20 million inguinal hernias are surgically corrected worldwide. For men, the lifetime risk of developing an inguinal hernia is estimated to be $27 \%$.

In the vast majority of cases an inguinal hernia is a clear-cut problem, presenting as a reducible groin swelling with a positive cough impulse (Valsalva manoeuvre), accompanied by discomfort or pain. Usually, the diagnosis is based upon history and physical examination, without the need for additional imaging. Additional imaging may only be required if there is clinical uncertainty. In these cases groin ultrasound (US) is considered to be the most suitable diagnostic modality. ${ }^{2}$

Not only the diagnosis of an inguinal hernia is often straight forward, the principle behind the treatment is equally simple. Since an inguinal hernia is not self-limiting, the treatment of all symptomatic hernias is an operative repair with reinforcement of the posterior inguinal wall. ${ }^{3}$ Asymptomatic or minimally symptomatic inguinal hernias may be managed with watchful waiting, since the risk of hernia-related emergencies (e.g. incarceration or strangulation) is low. ${ }^{2}$

In the past few decades the quality of inguinal hernia surgery has improved drastically. Recurrence rates have fallen below $5 \%$ after the introduction of mesh repair and post-operative pain rates have dropped since laparoscopy was introduced. Although there is wide variety of surgical techniques, patient- and hernia-related characteristics and local/national resources, laparoscopic techniques are gaining popularity worldwide. Provided that sufficient expertise is available, these techniques result in less chronic pain and an earlier return to normal activities. ${ }^{4-6}$

Now that technical improvements appear to have reached a plateau and post-operative pain and recurrence rates are at acceptably low levels, the interest concerning inguinal hernia repair appears to have shifted towards efficiency. Today, efficiency in health care is high on the political agenda, as health care costs keep rising while budgets are increasingly restricted. Multiple studies estimate that over $20 \%$ of global healthcare expenditure is wasteful, due to low-value medical practices that are either ineffective or cost more than the (equally effective) alternatives. ${ }^{7,8}$ Therefore, doctors are not only held responsible for improving quality of care, they are also obliged to pursue improvement of efficiency and eradicate waste. This can be achieved by simplifying care and administration, reducing errors and complications and avoiding redundant or low-value medical practice (e.g. operations in patients without complaints or unnecessary medical imaging). 
In order to improve efficiency in inguinal hernias, all steps in the chain have to be mapped out. From a patient making an appointment with the general practitioner (GP) through the surgical intake, the pre-operative screening, the surgical repair, and finally the post-surgical care. If all steps are critically scrutinized, efficiency may be improved through accelerating treatment, minimalizing hospital visits and providing a rapid return to work. Furthermore, preventing overuse of medical imaging or antibiotics, critically assessing the value of an operation, and further reducing complications with their associated costs, will all have an important role in the search for efficiency in inguinal hernias.

This thesis is composed of a variety of studies regarding efficiency from a high-volume hernia centre focusing on totally extra-peritoneal (TEP) inguinal hernia repair. In this centre over 1200 procedures are performed annually by 5 specialised hernia surgeons. The departments of surgery, radiology and anaesthesia are working closely together in the work-up of patients with inguinal problems.

\section{THESIS OUTLINE}

One of the pillars of efficiency is to simplify care and avoid unnecessary hospital visits. This may be accomplished by the single visit pathway, combining the traditionally separate preoperative evaluation, diagnostics and subsequent TEP inguinal hernia repair into one single visit. Recent studies have proven this approach to be efficient, with a high level of patient satisfaction. However, studies evaluating the impact of the single visit method on reducing total health care costs have never been published. In Chapter $\mathbf{2}$ the results are described of a cost-analysis comparing single visit and regular TEP repair in an employed and otherwise healthy population from both a hospital and societal point of view.

In light of avoiding unnecessary medical costs and low-value medical practice, it is important to focus on redundant medical imaging. It is well-known that the vast majority of inguinal hernias are primarily clinical diagnosis based on medical history \& physical examination. However, in daily practice general practitioners (GP), who are the initial contact for all medical matters (including groin complaints), often request or perform groin ultrasound (US) before referring to the surgeon.

It is questionable whether all these US are necessary. Reducing unnecessary diagnostics in primary care and their accompanying costs and waste of time are an important step in increasing efficiency. Chapter $\mathbf{3}$ contains a critical evaluation of the amount and impact of "first line" groin US-examinations prior to referral of patients to the surgical outpatient department.

Since US is widely used in case of ambiguous groin complaints (non-specific pain inguinal pain without a visible bulge), it is not uncommon to see patients with an inguinal hernia diagnosed with groin US, without any evident clinical features during physical examination. In these cases it is questionable whether such a subclinical inguinal hernia diagnosed on ultrasound truly underlies the complaint. Hence, a side-effect of ultrasound could be overdiagnosing which 
may prompt superfluous operations of inguinal hernias that would not have caused clinical symptoms during life if left untreated. All published data on groin ultrasound concern evident clinical inguinal hernias or symptomatic clinically occult hernias. Studies on the prevalence of inguinal hernias as an incidental finding on ultrasound have never been published. In Chapter 4 we determined the prevalence of an subclinical inguinal hernia among healthy, workingage men without groin complaints, in order to obtain insight into the probability that groin complaints are wrongly attributed to an inguinal hernia.

Although recurrence rates are low, recurrences still occur. The costs associated with recurrent hernias are often high due to pain medication, multiple visits to the GP and/or outpatient clinic, additional imaging, absence from work and re-operations. Understanding the etiology of an recurrent hernia and identify possible causes could help to increase efficiency and lower costs by further preventing recurrences. In Chapter 5 an 11-years analysis is presented of all reoperated groins for a suggested recurrence after TEP inguinal hernia repair $(n=137)$. Given the large number of operations (and subsequent reoperations) performed in the Dutch Hernia Clinic, a clear picture could be obtained of the various causes underlying recurrences after TEP inguinal hernia repair.

In the majority of cases, the clinical features of recurrent inguinal hernias are similar to those of primary inguinal hernias. However, diagnosing a recurrent hernia (after open repair) can be difficult. In the international guidelines for groin hernia management, physical examination (PE) combined with groin US is suggested as most suitable to confirm the diagnosis of a recurrent groin hernia. It is hitherto unknown to what extent this also applies to recurrences after endoscopic repair. In Chapter $\mathbf{6}$ we aimed to evaluate the positive predictive value (PPV) of PE and appraise the added value of US in recurrent inguinal hernias after totally extraperitoneal (TEP) inguinal hernia repair. If the PPV of PE in recurrent inguinal hernias is comparable to that of a primary inguinal hernia, this may reduce uncertainty among surgeons and subsequently lead to a decrease in superfluously requested medical imaging.

Besides recurrences, post-operative pain, or chronic postoperative inguinal pain (CPIP) remains an important complication after hernia repair. Although post-operative pain rates are at low levels, further decreasing CPIP would have a positive impact on the efficiency by reducing the amount of outpatient visits, pain medication, workplace absenteeism and re-operations. One of the risk factors for developing CPIP is young age. ${ }^{2}$ To reduce postoperative pain, several authors suggest that an open herniotomy without mesh and/or plasty might be preferable in young people. However, there is no consensus with regard to a clear definition of 'young age'. Furthermore, the risk factor 'young age' is primarily based on studies concerning open hernia repair. In Chapter 7 we determined whether young adults (18-30 years) are more prone to experience CPIP after TEP repair compared to adults older than 30 .

Although laparoscopic techniques are suggested as most suitable in the majority of cases, open hernia repair (especially Lichtenstein) is still widely performed. Where a laparoscopic repair is considered a clean surgical technique with low rates of postoperative wound infections, the question whether to use antibiotic prophylaxis in open hernia repair remains a matter of discussion. ${ }^{9}$ 
Erroneously refraining from administer antibiotic prophylaxis in contaminated surgical procedures may lead to wound infections and entails additional costs, whereas unnecessary administer antibiotic prophylaxis in clean surgical procedures can be considered as overtreatment. In Chapter 8 we reviewed the available evidence from RCTs assessing the effect of antibiotic prophylaxis on the prevention of wound infections after open inguinal or femoral hernia repair in adults.

1. Kingsnorth A, LeBlanc K. Hernias: Inguinal and incisional. Lancet. 2003;362:1561-1571. doi:http://dx.doi. org/10.1016/s0140-6736\%2803\%2914746-0.

2. The Herniasurge Group (2018) International guidelines for groin hernia management. Hernia 22:1-165.

3. Bekker J, Keeman JN, Simons MP, Aufenacker TJ (2007) A brief history of the inguinal hernia operation in adults. Ned Tijdschr Geneeskd. 2007 Apr 21;151(16):924-31.

4. Eklund AS, Montgomery AK, Rasmussen IC et al. Low recurrence rate after laparoscopic (TEP) and open (Lichtenstein) inguinal hernia repair: a randomized, multicentre trial with 5-years follow-up. Ann Surg 2009; 249(1):33-8

5. Schmedt CG, Sauerland S, Bittner R (2005) Comparison of endoscopic procedures vs Lichtenstein and other open mesh techniques for inguinal hernia repair: a meta-analysis of randomized controlled trials. Surg Endosc 19(2):188-199.

6. Hamza Y, Gabr E, Hammadi H, Khalil R (2010) Four-arm randomized trial comparing laparoscopic and open hernia repairs. Int J Surg 8(1):25-28.

7. Herrera-Perez D, Haslam A, Prasad V. (2019) A comprehensive review of randomized clinical trials in three medical journals reveals 396 medical reversals. Elife. Jun 11;8, pii: e45183

8. Shrank W.H. Rogstad T.L. Parekh N. Waste in the US Health Care System. Estimated Costs and Potential for Savings

9. F.J. Sanchez-Manuel, J. Lozano-Garcia, J.L. Seco-Gil (2012) Antibiotic prophylaxis for hernia repair Cochrane Database Syst Rev, 15, p. CD003769 
One-stop routing for surgical interventions; a costanalysis of endoscopic groin repair; A cost-analysis of endoscopic groin repair

\author{
CV van Hessen \\ MM Roos \\ GWJ Frederix \\ EJMM Verleisdonk \\ GJ Clevers \\ PHP Davids \\ JPJ Burgmans
}




\section{ABSTRACT}

\section{Background}

Single visit totally extraperitoneal (TEP) inguinal hernia repair is an efficient service without impairment of safety or complication rate. Data on the economic impact of this approach are rare. The aim of this study was to compare the costs between the single visit (SV) TEP and the regular TEP in an employed healthy population from a hospital and societal point of view.

\section{Methods}

Retrospectively collected hospital costs and prospectively collected societal costs were obtained from patients treated between July 2016 and January 2018. Outcome measures consisted of all documented institutional care, productivity loss and medical consumption.

\section{Results}

For analysing the hospital costs a total of $116 \mathrm{SV}$ patients were matched to 116 regular patients. The hospital costs of a mean SV patient were $€ 1148.78$ compared to $€ 1242.84$ for a regular patient, with a mean difference of $€ 94.06$. Prospective analyses of $50 \mathrm{SV}$ patients and 50 regular patients demonstrated higher societal costs for a mean regular patient ( $€ 2$ 188.33) compared to a mean single visit patient ( $€ 1621.44$ ). The mean total cost difference between a SV TEP repair and a regular TEP repair equalled $€ 660.95$ corresponding to a $19.3 \%$ decrease in costs.

\section{Conclusions}

This comprehensive cost-analysis showed that in an employed, healthy population, the single visit TEP repair outprices the regular TEP repair, with savings of $€ 660.95$ per patient, reflecting a $19.3 \%$ decrease in costs. This routing is mainly interesting from a societal point of view as the difference is mainly impacted by a decrease in societal costs. 


\section{BACKGROUND}

In the current era where health care costs maintain to rise and the health-care budget is increasingly restricted, it is a challenge to attain maximal health benefit that is not only cost-effective, but also provides patient satisfaction. ' In order to achieve this aim and reflect interest of society on all stakeholder groups, clinical pathways are integrated with increasing frequency. ${ }^{2-4}$ Given that groin hernia repair is one of the most commonly performed surgical procedures (more than 20 million worldwide and an estimated 28000 procedures in the Netherlands annually ${ }^{5}$ ), a small improvement in care regarding hernia surgery can consequently lead to a big enhancement of health care outcomes. ${ }^{6,7}$

Nowadays several surgical specialties aim to achieve improvement for different surgical procedures, by combining the traditionally separate preoperative evaluation, diagnostics and subsequent operation into one single visit (one-stop shop). ${ }^{8-12}$ The hernia clinic of the Diakonessenhuis Utrecht/Zeist provides a corresponding pathway for inguinal hernias and started to offer single visit (SV) endoscopic totally extraperitoneal (TEP) hernia repair since 2010. Results of a recently conducted study have demonstrated that this service appears to be a suitable option, with a high level of patient satisfaction and without impairment of safety or complication rate. ${ }^{13}$

Reducing health care costs through accelerating treatment, minimalizing hospital visits, and providing a rapid return to work has a strong intuitive appeal, however studies on the actual impact of this approach on reducing total health care costs have never been published. The aim of this study was to widen the current knowledge of economic impact by assessing the costs of SV endoscopic TEP hernia repair compared to regular TEP hernia repair. In order to provide a comprehensive view, both hospital costs and costs outside the hospital (societal costs; consisting of productivity loss and medical consumption) were assessed.

\section{METHODS}

\section{Design}

The study was carried out in the Hernia Clinic of the Diakonessenhuis Zeist, a high-volume hernia centre focusing on TEP inguinal hernia repair. In this centre over 1200 procedures are performed annually, of which up to $15 \%$ involve SV repair.

This cost-analysis compared SV TEP hernia repair to regular TEP hernia repair and consisted of two analyses. In a retrospective analysis of patients treated between July 2016 and May 2017, the hospital costs were compared. In a prospective analysis of patients treated between July 2017 and January 2018, societal costs were compared. 


\section{Single Visit Routing}

Patients received information regarding the single visit TEP repair through the website of the hernia clinic or through their general practitioner (GP). When the patient contacted the hernia clinic, a telephone pre-operative screening (T-POS) assessing eligibility for SV TEP hernia repair was conducted by the department's secretary. After approval of eligibility for the SV procedure, patients filled out a questionnaire, which was sent to the anaesthesiology department as an electronic alternative for the pre-operative screening (E-POS). After the E-POS was approved, the surgical intake and TEP hernia repair were scheduled for the same day. Detailed data on the recruitment process and patient routing within the SV hernia clinic (Figure 1) have been described previously. ${ }^{13}$

Figure 1. Timeline cost analysis

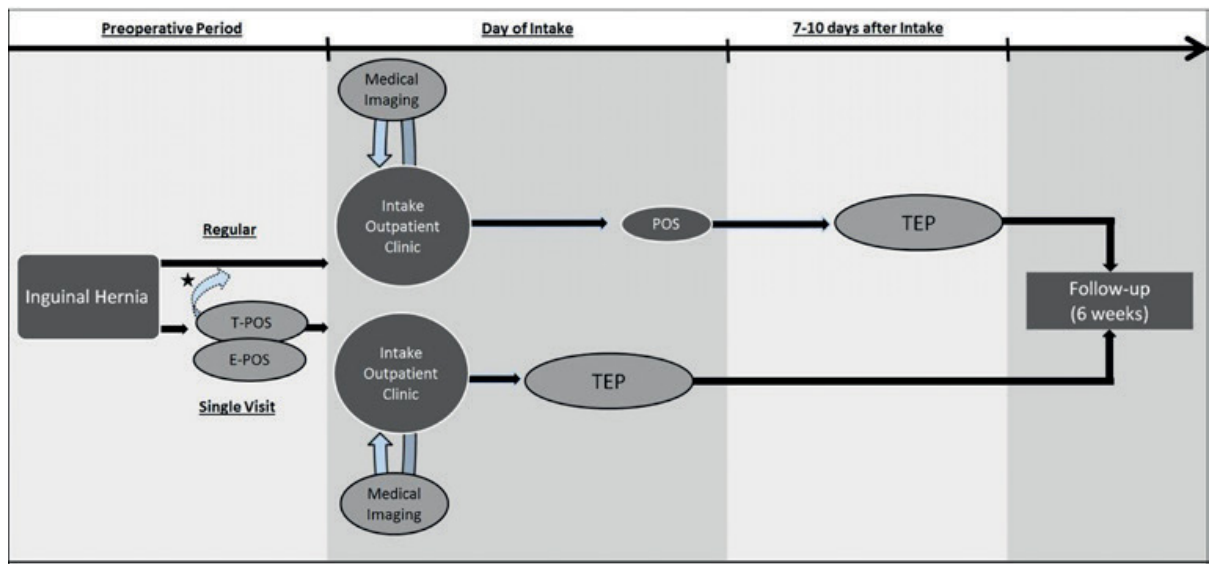

$\star$ Only if a patient appears to be not eligible based on pre-operative screening. T-POS: telephone preoperative screening (by the secretary), E-POS: electronic preoperative screening (by the physician assistant, POS: physical preoperative screening (anesthesiologi

\section{Regular Routing}

Patients referred through their GP for a regular TEP, first had their surgical intake at the outpatient clinic. If the surgeon confirmed a groin hernia, the pre-operative screening (POS) was conducted on the same day. After approval the TEP was planned within 7-10 days (Figure 1). 


\section{Patients}

The target population for this analysis consisted of employed men and women, aged between 18 and 65 years old with a primary unilateral inguinal hernia (Tab/e 1). Patients in the SV group in whom a bilateral hernia was diagnosed after physical examination of the surgeon, underwent bilateral hernia repair on the same day. For the hospital costs, all patients who underwent a single visit TEP between July 2016 and May 2017 were retrospectively identified and matched in terms of age, sex, BMI and number of bilateral hernias to patients who underwent a regular TEP procedure. As for the societal costs, patients who underwent a single visit TEP between July 2017 and January 2018 and all eligible patients who underwent a regular TEP repair in this period, were prospectively analysed.

Table 1. Eligibility Criteria Single Visit

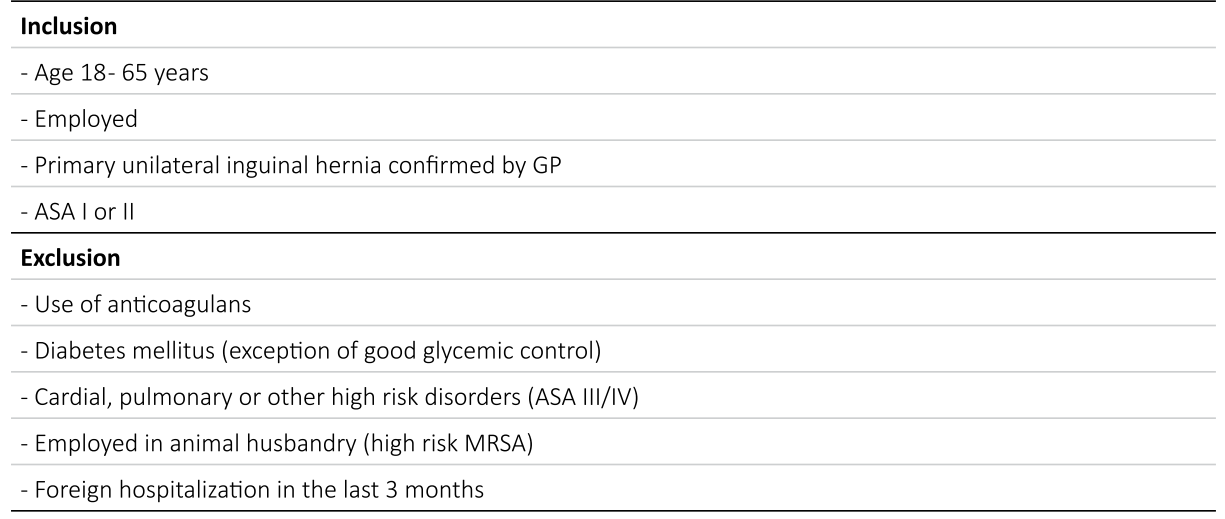

GP:General Practitioner, ASA:American Society of Anesthesiologists, MRSA:methicillin-resistant Staphylococcus Aureus

\section{Surgical procedure}

The applied surgical technique in all patients was the endoscopic TEP repair with implantation of a polypropylene mesh (Prolene ${ }^{\oplus}, 10 \times 15 \mathrm{~cm}$ ). Procedures were performed under general anaesthesia. Fixation of mesh was not routinely performed. Further surgical details on the TEP repair have been described in previous literature. ${ }^{14}$ Procedures were carried out by five experienced surgeons specialised in TEP hernia repair ( $>1000$ procedures per surgeon).The operation and the perioperative care were standardized and did not differ between the SV and the regular group.

\section{Post-operative management and follow-up}

There was no difference in post-operative management between the two cohorts compared. 
Discharge took place on the day of surgery, unless complications prohibited early discharge. At discharge, patients were advised to take pain medication during the first days and abstain from strenuous physical exercise during the first week. Follow-up took place by telephone, 6 weeks after surgery, according to a fixed schedule. In case of physical complaints an additional visit to the outpatient clinic was planned.

\section{Outcome measures}

The primary outcomes of this study were hospital costs and societal costs. The costs were valued, using a top-down approach in which all relevant cost components were identified and valued for the average patient by separating out costs from a comprehensive resource such as annual accounts. ${ }^{15}$

\section{Statistics}

Analyses were performed using SPSS statistical software, version 24 (IBM Corp, Armonk, NY). Descriptive statistics were used for baseline data. For comparison of all endpoints (not normally distributed), the Mann-Whitney $U$ analysis was used. A $p$ value of $<0.05$ (two-sided) was considered significant.

\section{Hospital costs}

To obtain standardized hospital cost estimates, the Dutch Manual for Costing was used for all documented care according to the International Classification of Diseases (ICD-10). ${ }^{16}$ The costs of an inpatient day included both direct costs (e.g. nursing costs and medical materials) and indirect costs (e.g. accommodation and overheads). For some specific SV costs, such as the T-POS and E-POS, no ICD-10 codes were assigned or no reference price was available. Hence it was necessary to calculate these costs based on the average time it took for a secretary (15 minutes) or physician assistant (5 minutes) to screen a patient. Furthermore, there was no reference price for the TEP hernia repair, therefore we took the published price of a recent study in a setting comparable to ours. ${ }^{17}$ The costs for medical staff members were calculated by multiplying the mean operative time of 20.3 minutes (as described in a previous publication ${ }^{13}$ ) with the standardized patient-related hourly wage of a Dutch medical specialist. ${ }^{16}$ Costs in euros per item are shown in Table 2. 
Table 2. Costs per item

\begin{tabular}{lc}
\hline Cost Item & Costs (€) \\
\hline Intake & 91,00 \\
\hline Groin ultrasound & 80,00 \\
\hline POS & 91,00 \\
\hline T-POS & 5,65 \\
\hline E-POS & 7,50 \\
\hline TEP repair & 484,90 \\
\hline Wage Surgeon & 39,00 \\
\hline Wage Anaesthesiologist & 39,00 \\
\hline Inpatient Day & 405,00 \\
\hline Telephone Consultation & 17,00 \\
\hline Physical Consultation & 91,00 \\
\hline
\end{tabular}

POS: preoperative screening, T-POS: telephone preoperative Screening, E-POS: electronic preoperative screening, TEP = totally extraperitoneal

\section{Societal costs}

The societal costs were based on productivity and medical consumption. Two validated questionnaires were used; the productivity cost questionnaire (iPCQ) and the medical consumption questionnaire (iPMQ). Regarding productivity, lost workdays and underperformance were extracted until 6 weeks after surgery and converted to costs based on the average (male) Dutch wage of $€ 37.90$ an hour (net income 2017). Given that every patient of the regular cohort had an intake in the weeks before the operation, half a lost workday was added for every regular patient. Medical consumption, travel expenses, pharmaceutical consumption and additional consultations of primary and secondary care were all extracted until 6 weeks after surgery and translated into costs. To obtain travel expenses, we multiplied the average distance to the hospital $(7 \mathrm{~km})$ by the tax-free reimbursement of $€ 0.19$ per kilometre and added $€ 3.00$ parking costs, based on the average car parking charges at hospitals. ${ }^{16}$ Pharmaceutical prices were based on current chain pharmacy cash pricing. ${ }^{18}$ We took the average of the highest and lowest price. As for the additional consultations, a standardized consultation with a general practitioner was set at $€ 33.00$ with a standardized traveling distance of $1,1 \mathrm{~km}$, which implies travel expenses of $(2.2 \times € 0.19=) € 0.42 .{ }^{16}$ Ambulance charges were set at $€ 515.00$. For some specific costs the reference price was unknown. In this case costs were estimated based on requested charges from health insurance companies and charges found on the internet. Thus, a consultation with a company doctor was set at $€ 80.00$, homeopathic care charges were set at $€ 88.00$ and blood tests in primary care setting were set at $€ 9.15$ (haemoglobin, white blood cells, C-reactive protein and erythrocyte sedimentation rate). 


\section{RESULTS}

Hospital costs

A total of 116 single visit patients were matched to 116 regular patients. The majority of patients in both groups were male (Table 3). The median age, Body Mass Index (BMI) and American Society of Anesthesiologists (ASA) classification were similar between both groups.

The total hospital costs for the SV TEP group were $€ 133259.30$ whereas the total hospital costs for the regular TEP group were $€ 144$ 169.90, with a difference of $€ 10910.60$ (Table 4). This equates to a mean SV TEP of $€ 1148.78$ ( $€ 1072.05$ to $€ 1650.05$ ) and a mean regular TEP of $€ 1242.84$ ( $€ 1149.90$ to $€ 1823.90$ ) with a mean difference of $€ 94.06$ per patient, reflecting a $7.6 \%$ decrease in costs in favour of the SV analysis. The foremost causes of this decrease are the pre-operative screening, inpatient days and telephone consultations (Figure 2).

A standard SV TEP, without complications or extra consultations costed €1 089.05. The additional costs of the mean analysed SV TEP therefore equalled $€ 59.73$. A total of 32 patients (27.6\%) made more hospital costs than the standard costs per SV patient, 72 patients (62.1\%) were equal to the standard and in 12 patients (10.3\%) less hospital costs were made (due to absence of follow-up by telephone after 6 weeks). A standard regular TEP, without complications or extra consultations costed $€ 1$ 166.89. The additional costs of the mean analysed regular TEP therefore equalled $€ 75.95$. A total of 45 patients (38.8\%) made more hospital costs, 61 patients (52.6\%) were equal to the standard and in 10 patients (8.6\%) less hospital costs were made (due to absence of follow-up by telephone after 6 weeks). 
Table 3. Baseline characteristics of 232 retrospectively analysed patients (hospital costs)

\begin{tabular}{|c|c|c|}
\hline & $\begin{array}{c}\text { Single-visit } \\
(n=116)\end{array}$ & $\begin{array}{l}\text { Regular } \\
(n=116)\end{array}$ \\
\hline Male, $n(\%)$ & $114(98)$ & $114(98)$ \\
\hline Age (years) & $50(40-57)$ & $48(42-55)$ \\
\hline BMI $\left(\mathrm{kg} / \mathrm{m}^{2}\right)$ & $24(23-27)$ & $25(23-26)$ \\
\hline \multicolumn{3}{|l|}{ ASA, n (\%) } \\
\hline । & $97(84)$ & $97(84)$ \\
\hline II & $19(16)$ & $19(16)$ \\
\hline \multicolumn{3}{|l|}{ Smoking, n (\%) } \\
\hline Current smoker & $17(15)$ & $23(20)$ \\
\hline Non-smoker & $92(81)$ & $87(76)$ \\
\hline Ex-smoker & $5(4)$ & $5(4)$ \\
\hline \multicolumn{3}{|l|}{ Occupation, n (\%) } \\
\hline Intense physical work & $30(27)$ & $35(30)$ \\
\hline Light intensity work & $33(29)$ & $23(20)$ \\
\hline Sedentary work & $45(40)$ & $54(47)$ \\
\hline Different & $5(4)$ & $2(2)$ \\
\hline \multicolumn{3}{|c|}{ Location of hernia, $n(\%)$} \\
\hline Unilateral (left) & $51(44)$ & $55(47)$ \\
\hline Unilateral (right) & $60(52)$ & $56(48)$ \\
\hline Bilateral & $5(4)$ & $5(4)$ \\
\hline \multicolumn{3}{|l|}{ Hernia type, n (\%) } \\
\hline Lateral & $87(75)$ & $85(73)$ \\
\hline Medial & $17(15)$ & $24(21)$ \\
\hline Femoral & $1(1)$ & $1(1)$ \\
\hline Medial (Bilateral) & $4(3)$ & $3(3)$ \\
\hline Lateral (Bilateral) & $1(1)$ & $1(1)$ \\
\hline Medial \& Lateral & $5(4)$ & $1(1)$ \\
\hline Lateral \& Femoral & $1(1)$ & $0(0)$ \\
\hline
\end{tabular}

Continuous data are presented as median (interquartile range). BMI: body mass index; ASA:American Society of Anesthesiologists 
Table 4. Uncomplicated and analysed hospital costs of a single-visit TEP and regular TEP

\begin{tabular}{|c|c|c|c|c|c|}
\hline & \multicolumn{2}{|c|}{ Single-visit } & \multicolumn{2}{|c|}{ Regular } & \multirow{2}{*}{$\begin{array}{l}\text { Difference in } \\
\text { analysed costs } \\
\text { (Regular - SV) }\end{array}$} \\
\hline & $\begin{array}{l}\text { Uncomplicated } \\
\text { costs }\end{array}$ & $\begin{array}{l}\text { Analysed } \\
\text { costs }\end{array}$ & $\begin{array}{l}\text { Uncomplicated } \\
\text { costs }\end{array}$ & $\begin{array}{l}\text { Analysed } \\
\text { costs }\end{array}$ & \\
\hline Intake & $€ 10.556,00$ & $€ 10.556,00$ & $€ 10.556,00$ & $€ 10.556,00$ & $€ 0,00$ \\
\hline Ultrasound & $€ 0,00$ & $€ 80,00$ & $€ 0,00$ & $€ 480,00$ & $€ 400,00$ \\
\hline POS & $€ 0,00$ & $€ 0,00$ & $€ 10.556$ & $€ 10.556$ & $€ 10.556$ \\
\hline E-POS & $€ 870,00$ & $€ 870,00$ & $€ 0,00$ & $€ 0,00$ & $-€ 870,00$ \\
\hline T-POS & $€ 655,40$ & $€ 655,40$ & $€ 0,00$ & $€ 0,00$ & $-€ 655,40$ \\
\hline TEP repair & $€ 56.248,40$ & $€ 56.248,40$ & $€ 56.248,40$ & $€ 56.248,40$ & $€ 0,00$ \\
\hline Wage surgeon & $€ 4.553,00$ & $€ 4.553,00$ & $€ 4.553,00$ & $€ 4.553,00$ & $€ 0,00$ \\
\hline Wage anesthesiologist & $€ 4.553,00$ & $€ 4.553,00$ & $€ 4.553,00$ & $€ 4.553,00$ & $€ 0,00$ \\
\hline Inpatient day & $€ 46.980,00$ & $€ 52.650,00$ & $€ 46.980,00$ & $€ 55.890,00$ & $€ 3.240,00$ \\
\hline Blood test & $€ 0,00$ & $€ 65,00$ & $€ 0,00$ & $€ 265,00$ & $€ 200,00$ \\
\hline Telephone consultation & $€ 1.972,00$ & $€ 1.938,00$ & $€ 1.972,00$ & $€ 2.754,00$ & $€ 816,00$ \\
\hline Physical consultation & $€ 0,00$ & $€ 1.092,00$ & $€ 0,00$ & $€ 1.183,00$ & $€ 91,00$ \\
\hline Total costs & $€ 126.387,80$ & $€ 133.260,80$ & $€ 135.418,40$ & $€ 147.038,40$ & $€ 13.777,60$ \\
\hline Mean costs & $€ 1.089,55$ & $€ 1.148,80$ & $€ 1.167,40$ & $€ 1.267,60$ & $€ 118,80$ \\
\hline
\end{tabular}

TEP: totally extraperitoneal, POS: preoperative screening, T-POS: telephone preoperative screening, E-POS: electronic preoperative screening, SV: single-visit 


\section{Societal costs}

A total of 50 eligible SV TEP patients were compared to 50 eligible regular TEP patients. The majority of patients in both groups were male (Table 5). The median age, BMI and ASA were similar.

Figure 2. Mean institutional costs

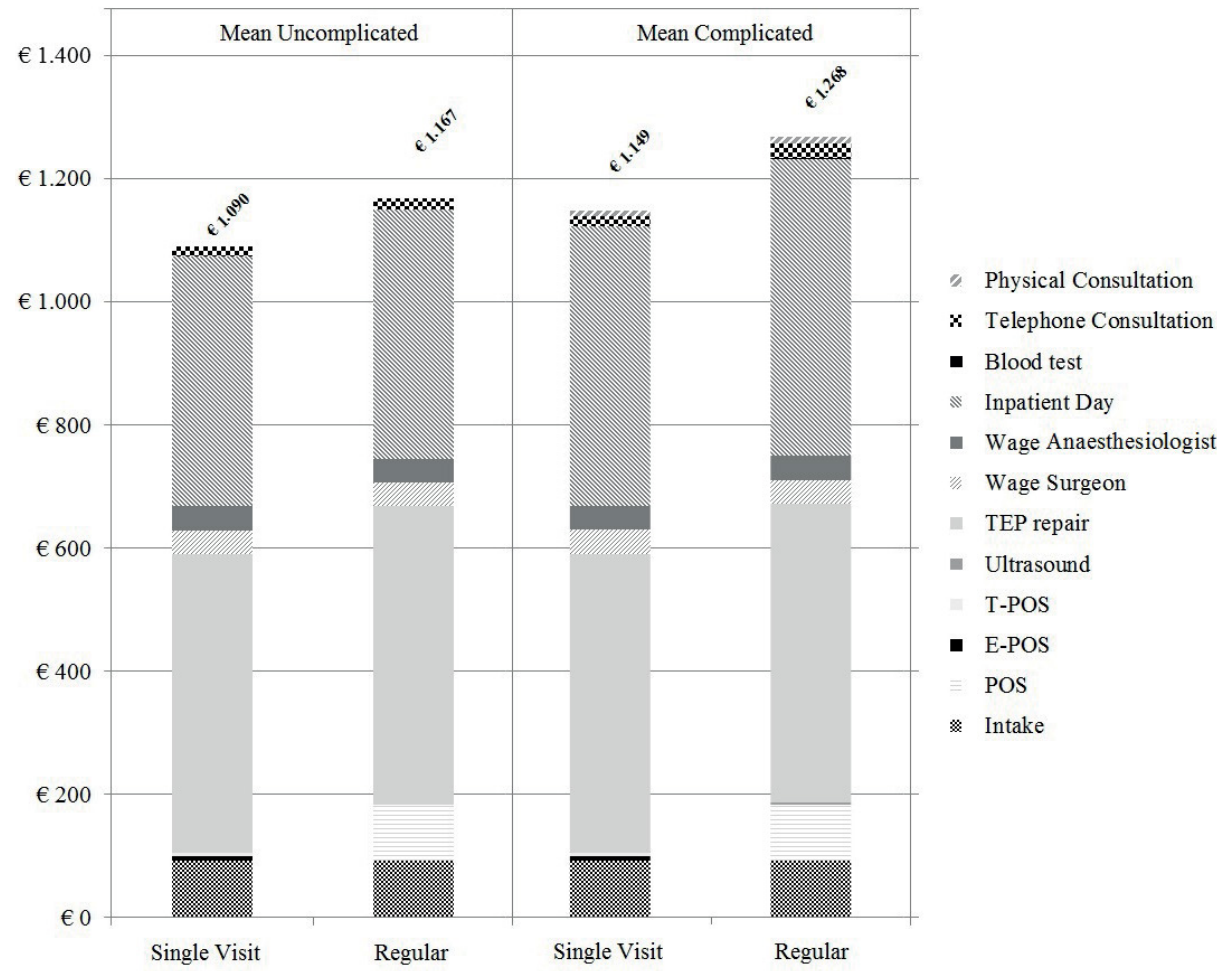

TEP: totally extraperitoneal, T-POS: telephone preoperative screening, E-POS: electronic preoperative screening

\section{Lost workdays and underproductivity}

Twenty-seven patients (54\%) in the single visit cohort indicated to have missed at least one day in the 6 weeks following the operation. This led to a total of 242.6 lost workdays including the day of operation, resulting in an amount of $€ 73$ 563.90. In the SV cohort, 14 patients (28\%) reported 128 under-productive days, with a mean of $83 \%$ productivity. This equalled 22.2 lost workdays, resulting in an amount of $€ 6$ 731.04. In the regular cohort, 19 patients (38\%) reported lost workdays after the operation, resulting in a total of 301 lost workdays (including day of operation \& day of intake), which equalled an amount of $€ 91$ 263.20. A total of 130 under-productive days were reported by 14 patients (28\%) in the regular group. With a mean productivity of $60 \%$, this resulted in 52.2 lost workdays, equalling an amount of $€ 15827.04$. 
Table 5. Baseline Characteristics of 100 prospectively analysed patients (costs outside hospital)

\begin{tabular}{|c|c|c|}
\hline & $\begin{array}{l}\text { Single-visit } \\
(n=50)\end{array}$ & $\begin{array}{c}\text { Regular } \\
(n=50)\end{array}$ \\
\hline Male, $\mathrm{n}(\%)$ & $49(98)$ & $46(92)$ \\
\hline Age (years) & $53(46-58)$ & $57(44-63)$ \\
\hline BMI $\left(\mathrm{kg} / \mathrm{m}^{2}\right)$ & $24(22-27)$ & $24(23-26)$ \\
\hline \multicolumn{3}{|l|}{ ASA, n (\%) } \\
\hline । & $39(78)$ & $22(44)$ \\
\hline$\|$ & $11(22)$ & $28(56)$ \\
\hline \multicolumn{3}{|l|}{ Smoking, n (\%) } \\
\hline Current smoker & $5(10)$ & $11(22)$ \\
\hline Non-smoker & $41(82)$ & $33(66)$ \\
\hline Ex-smoker & $4(8)$ & $6(12)$ \\
\hline \multicolumn{3}{|l|}{ Occupation, n (\%) } \\
\hline Intense physical work & $12(24)$ & $14(28)$ \\
\hline Light intensity work & $11(22)$ & $8(16)$ \\
\hline Sedentary work & $27(54)$ & $28(56)$ \\
\hline \multicolumn{3}{|c|}{ Location of hernia, n (\%) } \\
\hline Unilateral (left) & $16(32)$ & $17(34)$ \\
\hline Unilateral (right) & $34(68)$ & $33(66)$ \\
\hline Bilateral & $0(0)$ & $0(0)$ \\
\hline \multicolumn{3}{|l|}{ Hernia type, n (\%) } \\
\hline Lateral & $40(80)$ & $32(64)$ \\
\hline Medial & $6(12)$ & $10(20)$ \\
\hline Femoral & $2(4)$ & $3(6)$ \\
\hline Medial \& Lateral & $2(4)$ & $5(10)$ \\
\hline
\end{tabular}

Continuous data are presented as median (interquartile range), BMI: body mass index, ASA: American Society of Anesthesiologists

\section{$\underline{\text { Travel costs }}$}

The total number of hospital visits (including intake and POS) for the SV cohort was 51 in contrast to 103 for the regular cohort. The corresponding unalloyed travel costs were $€ 135.66$ and $€ 273.98$ respectively. By adding the parking costs, this resulted in total travel costs of $€ 282.66$ for the SV group and $€ 582.98$ for the regular group.

\section{Pharmaceutical consumption}

Of the SV cohort 36 (72\%) patients consumed medication in the 6 weeks following surgery. Of the regular cohort 35 patients (70\%) used medication. The drugs consumed in response to the inguinal hernia repair were paracetamol, non-steroidal anti-inflammatory drugs (NSAID's) in 
combination with proton-pump inhibitors and laxatives. Based on the current chain pharmacy cash pricing, a total amount of $€ 73.18$ was consumed by the single visit cohort and an amount of $€ 80.44$ by the regular cohort.

\section{Additional consultations}

Of the SV cohort 5 patients (10\%) consulted their GP (€167.10), 1 patient (2\%) consulted his company doctor (€88.00) and 1 patient (2\%) had 2 homeopathy consultations (€160,00). As for the regular cohort, 13 patients (26\%) consulted their GP (€434.46), 8 patients (16\%) visited their company doctor (€704.00), 1 patient (2\%) had his blood tested in primary care setting (€9.15) and 1 patient (2\%) used an ambulance due to severe constipation (€515.00).

\section{Mean societal costs}

To sum up, the total societal costs were €81 071.88 for the SV cohort and $€ 109416.27$ for the regular cohort. Hence, a mean SV patient costed $€ 1621.44$ (€308.86 to $€ 9598.28$ ) from a societal perspective whereas a regular patient costed $€ 2188.33$ ( $€ 466.12$ to $€ 7442.00$ ) from this perspective (Figure 3) (Tab/e 6), with a difference of $€ 566.89$, reflecting a $25.9 \%$ decrease in costs in favour of the SV analysis.

Table 6. Analysed societal costs of a single-visit TEP and regular TEP

\begin{tabular}{lrrr}
\hline & Single-visit & Regular & $\begin{array}{c}\text { Difference in analysed } \\
\text { costs (Regular - SV) }\end{array}$ \\
\cline { 2 - 4 } Lost workdays & $€ 73.980,80$ & Costs (€) & $€ 17.282,40$ \\
\hline Under-productivity & $€ 6.731,04$ & $€ 15.827,04$ & $€ 9.096,00$ \\
\hline Travel costs & $€ 135,66$ & $€ 279,30$ & $€ 143,64$ \\
\hline Parking costs & $€ 153,00$ & $€ 315,00$ & $€ 162,00$ \\
\hline Pharmaceutical consumption & $€ 73,20$ & $€ 77,80$ & $€ 4,60$ \\
\hline GP consultation & $€ 167,10$ & $€ 434,46$ & $€ 267,36$ \\
\hline Company doctor & $€ 88,00$ & $€ 704,00$ & $€ 616,00$ \\
\hline Homeopathy consultation & $€ 160,00$ & $€ 0,00$ & $-€ 160,00$ \\
\hline Blood test primary care & $€ 0,00$ & $€ 33,00$ & $€ 33,00$ \\
\hline Ambulance & $€ 0,00$ & $€ 515,00$ & $€ 515,00$ \\
\hline Total & $€ 81.488,80$ & $€ 109.448,80$ & $€ 1.581,60$ \\
\hline Mean & $€ 1.629,78$ & $€ 2.188,98$ & $€ 559,20$ \\
\hline
\end{tabular}

TEP: totally extraperitoneal, GP: general practitioner, SV:single-visit 
Figure 3. Mean societal costs

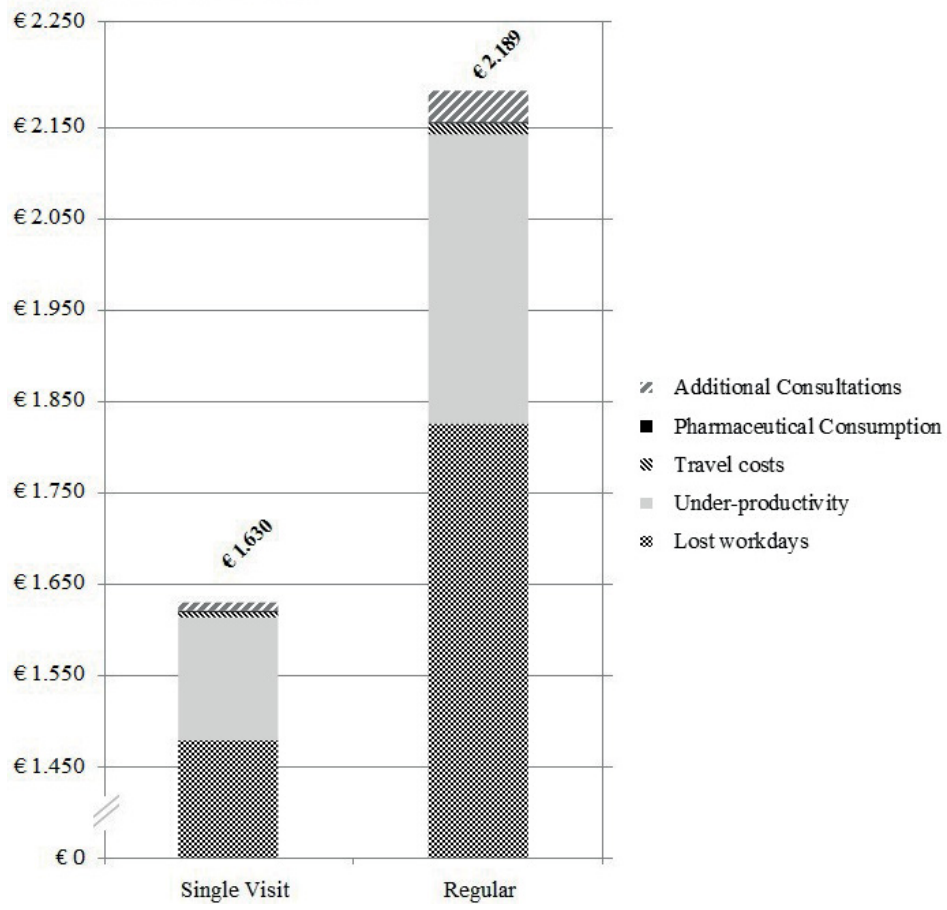

Total cost-analysis

By combining hospital and societal costs, a mean SV patient costed $€ 2770.22$. From this same comprehensive point of view a regular patient costed $€ 3431.17$. The mean difference between both pathways therefore equalled $€ 660.95$ which corresponded to a $19.3 \%$ decrease in costs in favour of the SV analysis.

\section{DISCUSSION}

The aim of this study was to provide a comprehensive view on the cost savings of the SV routing and to widen the current knowledge of economic impact. In an employed, healthy population, SV TEP inguinal hernia repair results in a $19.6 \%$ decrease in costs and offers cost savings of $€ 678.00$ per patient. These study results demonstrate that the SV endoscopic inguinal hernia repair outprices the regular TEP inguinal hernia repair for employed healthy patients and makes it an interesting alternative for the common pathway. The biggest cost reduction can be achieved within the societal costs. To our knowledge, this is the first costanalysis of single visit routing analysing both hospital and societal costs. 
The mean difference in hospital costs was $€ 118.80$ per patient, reflecting a $9.4 \%$ decrease in costs in favour of the SV procedure. This correlates fairly well with previous publications and further supports the idea of increased accessibility along with boosting hospital related productivity. Several studies have demonstrated lower costs in the one-stage surgery compared to its regular counterpart, without impairment of safety or complication rate. ${ }^{19-22}$ However, cost-items were not specified or poorly estimated. Olson et al, who compared the single visit paediatric ambulatory surgical procedures (SVS) with common surgery (CS), estimated that SVS had higher costs than common surgery (CS). ${ }^{23}$ With a total hospital reimbursement of $\$ 810878$ for the SVS group $(n=90)$ and $\$ 776762$ for the CS group $(n=90)$, this equalled an increase of $4.4 \%$ and contrasts our findings. The explanation may be sought in the different patient population consisting of children and same-day cancellation rate, which was higher in SVS group due to incorrect diagnoses, need for further testing or ability to do the procedure in clinic rather than the operating room. This high cancellation rate did not apply for our clinic. ${ }^{13}$

The main cause of lower hospital costs found in this study is the difference in POS between a regular (in hospital POS) and SV (T-POS and E-POS) procedure. Given that since 2005 the Dutch financing system gradually changed to a system with market competition, the findings can be of great interest for hospitals performing the TEP repair. ${ }^{24}$ However, this alternative POS may even be extrapolated to all healthy patients (ASA I or II) with an operation on the horizon.

The inequality of hospital costs cannot only be explained by the pathway itself and its alternative POS but is also a result of the difference in physical- and telephone consultations. Given the fact that the surgical procedure, perioperative- and postoperative care were standardized and did not differ between the SV and the regular group, an explanation for the difference in health care consumption might be found in the patient characteristics or the personality type. This, however, applies even more to the patient and societal perspective and will be discussed later on.

Despite the fact that the difference in hospital costs between the SV and regular treatment is small, big cost savings can be achieved in high volume settings and by implementing this method in day-care surgery for other procedures.

From a societal point of view, the mean difference in costs was $€ 566.89$, reflecting a $25.9 \%$ decrease in costs in favour of SV TEP repair. This correlates with the findings of Olson et al, who found family cost savings of $\$ 188.00$ for the SVS compared to CS, reflecting a $44.5 \%$ decrease in costs. ${ }^{23}$ However, Olson et al. used the mean household income and the mean travel distance (which differed between groups) instead of standardised values, which may explain the bigger difference in costs.

The inequality of costs is mainly a result of the increased number of lost workdays and hospital visits, with the associated travel expenses. Given the one-stage surgery-routing of the single visit group, this seems to be a natural consequence. An extra hospital visit for the intake not only means additional travel expenses, but also additional (partially) lost workdays. 
However, the inequality exceeded our expectations. It seems that the SV-patient has a smaller demand for health care and consequently has a reduced number of lost workdays. In line with the difference in hospital costs, an explanation can be found in the patient characteristics and personality type. It is plausible that the SV pathway especially attracts those who are the "go-getter" type of patient. The patient type who has looked into the matter carefully, gets the possible advantages and disadvantages and is understanding in the face of adversity. It is not unlikely that the health consumption to some extent is built much more around intrinsic motivation than the actual need. Negative experiences, passivity, hostility and pessimism can all contribute to health care consumption and therefore can be related to various negative health outcomes, whereas optimistic control has a significant positive impact on various health indicators, although the existing literature is not conclusive on this subject. ${ }^{25-28}$

Another possible explanation that can justify the inequality is the difference in occupation between the two groups compared. The number of patients with intense physical work is higher in the regular group whereas the number of patients with light intensity work is lower. At discharge, patients were advised to abstain from strenuous physical exercise for the first week, which consequently applies more to patients with intense physical work. Given that work has long been associated with key components of mental health, it is also plausible that early return to work has a positive effect on recovery by distraction. ${ }^{29}$ Hence, the negative effect of not working and sitting at home could have a wider impact on the regular group.

It is not inconceivable that a number of limitations could have influenced the study results. These limitations indicate the difficulty of collecting data on a comprehensive cost-analysis. The time horizon over which these costs were evaluated, was 6 weeks after surgery and started from the day of intake. Hence, medical consumption in the period before the intake was not taken into account. This underestimate of total health care costs is made deliberately, given the difficulties of retrospectively retrieving all medical records from GP's, other hospitals or paramedical care. In line with the personality types, one can expect a higher number of doubters and second opinions in the regular group, thus it is not unlikely this underestimation applies particularly to this group.

A general limitation of this cost-analysis is the high volume context. As the study is carried out at the Hernia Clinic of the Diakonessenhuis Zeist/Utrecht, the largest Dutch hernia centre focusing on TEP repair, with a well-organised SV routing including experienced secretaries, this might not be applicable for every hospital. The same goes for some of the hospital costs (e.g. the TEP repair), which are relatively low. Due to the differences between hospitals in costs of OR-time and inpatients days, one has to consider the results as a comprehensive estimation.

Another limitation of the prospective analysis, is the fact that we worked with a voluntary response sample. Not every applicable patient finished and returned their questionnaire, which may have led to a voluntary response bias, due to the fact that it probably oversamples patients who have strong opinions. Together with the small sample size, the voluntary responses may have also led to large costs spreads.

Despite the fact that this study found relatively small cost savings for hospitals, the SV routing can be of great interest for high volume hospitals performing the TEP repair and can be 
extrapolated to other commonly performed surgical procedures. By changing the pathway within a hospital, the effect outside the hospital will be considerably higher. Therefore both employers and employees will benefit most from changing the hospital patient routing with a $25.9 \%$ decrease in costs.

\section{REFERENCES}

1. Weinstein M, Stason W. (1977) Foundations of cost-effectiveness analysis for health and medical practices. New England Journal of Medicine. 296(13):716-21.

2. Rotter T, Kinsman L, James E, Machotta A, Gothe H, Willis J, Snow P, Kugler J (2010) Clinical pathways: effects on professional practice, patient outcomes, length of stay and hospital costs. Cochrane Database Syst Rev CD006632.

3. Shitao Yang, Curtis P. McLaughlin, Robert W. Vaughan, John J. Aluise (1992) Factory Focus in Hospitalowned Ambulatory Surgery. International Journal of Service Industry Management, 3(4):63-75

4. Muller MK, Dedes KJ, Dindo D, Steiner S, Hahnloser D, Clavien PA (2009) Impact of clinical pathways in surgery. Langenbecks Arch Surg 394:31-39

5. Statline, Centraal Bureau voor statistiek

6. Joh HJ, Moon IS, Park HR, Kim NC, Yang S (2003) The effects of the critical pathway for inguinal hernia repair. Yonsei Med J 44:81-8

7. Uchiyama K, Takifuji K, Tani M, Onishi H, Yamaue H (2002) Effectiveness of the clinical pathway to decrease length of stay and cost for laparoscopic surgery. Surg Endosc 16(11):1594-7

8. Tagge EP, Hebra A, Overdyk F, Burt N, Egbert M, Wilder A, Williams A, Roland P, Othersen HB Jr (1999) OneStop Surgery: Evolving Approach to Pediatric Outpatient Surgery. J Pediatr Surg 34: 129-132

9. Gilmartin J, Chin H, Leonard P (2009) Pre-admission clinics in day surgery. The 'one-stop shop': tools and methods for practice improvement and service development. Prac Dev Health Care 8:239-252

10. Kashani S, Muhtaseb M, Robinson F (2005) Analysis of 'one-stop' referral system for ophthalmic minor operations by GPs. Br J Gen Pract 55(510): 53-54.

11. Barnett SJ, Frischer JS, Gaskey JA, Ryckman FC, von Allmen D (2011) Pediatric hernia repair: 1-stop shopping. J Pediatr Surg 47(1):213-6

12. Bismil M, Bismil Q, Harding D, Harris P, Lamyman E, Sansby L (2012) Transition to total one-stop wide-awake hand surgery service-audit: a retrospective review. J R Soc Med Sh Rep 3: 23

13. Voorbrood CE, Burgmans JP, Clevers GJ, Davids PH, Verleisdonk EJ, Schouten N, van Dalen T (2015) One- 
stop endoscopic hernia surgery: efficient and satisfactory. Hernia 19(3):395-400

14. Sinha R, Sharma N, Dhobal D, Joshi M (2006) Laparoscopic Total extraperitoneal repair versus anterior preperitoneal repair for inguinal hernia. Hernia 10(2):187e91

15. Tan SS. (2009) Microcosting in economic evaluations: Issues of accuracy, feasibility, consistency and generalisability. Instituut Beleid en Management Gezondheidszorg (iBMG) 1st ed. Rotterdam: Erasmus Universiteit Rotterdam.

16. Hakkaart-van Roijen L, Van der Linden N, Bouwmans CAM, Kanters T, Tan SS (2015) Dutch Manual for Costing: METHODS and Reference Prices for Economic Evaluations in Healthcare. Institute for Medical Technology Assessment (IMTA) Rotterdam.

17. Eklund A. Carlsson P, Rosenblad A, Montgomery A, Bergkvist L, Rudberg C (2010) Long-term costminimization analysis comparing laparoscopic with open (Lichtenstein) inguinal hernia repair. Britisch Journal of Surgery 97: 765-771

18. Zorginstituut Nederland. Oktober 2017.URL:https://www.medicijnkosten.nl/

19. Putnis S, Merville-Tugg R, Atkinson S (2004) 'One-stop' inguinal hernia surgery - day-case referral, diagnosis and treatment. Ann R Coll Surg Engl 86:425-7.

20. Kashani S, Muhtaseb M, Robinson F (2005) Analysis of 'one-stop' referral system for ophthalmic minor operations by GPs. Br J Gen Pract 55(510): 53-54.

21. Barnett SJ, Frischer JS, Gaskey JA, Ryckman FC, von Allmen D (2011) Pediatric hernia repair: 1-stop shopping. J Pediatr Surg 47(1):213-6

22. Bismil M, Bismil Q, Harding D, Harris P, Lamyman E, Sansby L (2012) Transition to total one-stop wide-awake hand surgery service-audit: a retrospective review. J R Soc Med Sh Rep 3: 23.

23. Olson JK, Deming LA, King DR, Rager TM, Gartner S, Huibregtse N, Moss RL, Besner GE (2017) Single visit surgery for pediatric ambulatory surgical procedures: a satisfaction and cost analysis. J Pediatr Surg. SOO223468(17)30643-7

24. Krabbe-Alkemade Y.J.F.M., Groot TL, Lindeboom M (2017), Competition in the Dutch hospital sector: an analysis of health care volume and cost. Eur J Health Econ 18(2):139-153

25. Vollrath ME, editor. Handbook of personality and health. New York (NY): Wiley; 2006

26. Dickerson SS, Kemeny ME (2004) Acute stressors and cortisol responses: a theoretical integration and synthesis of laboratory research. Psychol Bull. 130:355-91. doi: 10.1037/0033-2909.130.3.355.

27. Steptoe A. (1993) The significance of personal control in health and disease. In: Steptoe A, Appels A, editors. Stress, personal control and health. Chichester (UK): Wiley; p. 309-18. 
28. Scharloo M, Kaptein AA. (1997) Measurement of illness perceptions in patients with chronic somatic illness: a review. In: Petrie KJ, Weinman J, editors. Perceptions of health and illness: current research applications. London: Harwood; p. 103-35.

29. Isaksson K. Unemployment, mental health and the psychological functions of work in male welfare clients in Stockholm. Scand J Soc Med. 1989;17(2):165-9 


\title{
Overuse of groin ultrasound for diagnosing inguinal hernias in daily practice
}

\author{
CV Hessen \\ MM Roos \\ FBM Sanders \\ EJMM Verleisdonk \\ A de Vries \\ GJ Clevers \\ PHP Davids \\ JPJ Burgmans
}

Submitted 


\section{ABSTRACT}

\section{Background}

Inguinal hernias are primarily clinical diagnoses. In case of equivocal findings on physical examination, imaging may be required. The aim of this study was to evaluate the utilization and influence of groin ultrasound in patients referred to the surgical outpatient clinic.

\section{Methods}

All adult patients that underwent groin ultrasound for suspicion of inguinal hernia upon request of their general practitioner (GP) that were referred to the surgical outpatient department of a Dutch hernia clinic between July 2016 and July 2017, were studied retrospectively. Outcomes of ultrasound and clinical assessment were compared. In case of a discrepancy between ultrasound and clinical findings, influence of ultrasound on surgical decision-making was assessed.

\section{Results}

In 361 out of 1371 patients (26\%), groin ultrasound was performed through the GP, involving 399 groins. Findings of 374 ultrasounds (94\%) were positive for inguinal hernia and 25 ultrasounds were negative (6\%). On physical examination, in 316 groins (79\%) a clinically apparent inguinal hernia was reported and in 69 groins (17\%) no hernia could be objectified. In 14 groins (4\%) the diagnosis was inconclusive. Discrepancy between ultrasound and physical examination existed in 58 groins (15\%). In this group 14 cases (24\%) were (surgically) treated based on ultrasound outcomes.

\section{Conclusions}

Groin ultrasound was performed upon request of the GP in 26\% of all referred patients, of which $79 \%$ had an inguinal hernia upon physical examination. In case of discrepancy between ultrasound and physical examination outcomes, (surgical) treatment was based on physical examination in $76 \%$. 


\section{INTRODUCTION}

Inguinal hernia is a frequently seen condition, and in many patients elective repair is performed. Approximately $95 \%$ of inguinal hernias can be diagnosed by physical examination, based on the classical presentation of a reducible groin swelling with a positive cough impulse (Valsalva manoeuvre), sometimes accompanied with pain.,2 Anamnestic history of groin swelling can contribute to the diagnosis as well. Only in case of vague groin swelling and diagnostic uncertainty, poor localization of swelling, intermittent swelling not present at time of physical examination or obscure groin complaints without swelling, imaging may be required. ${ }^{2}$

In case of equivocal clinical findings ultrasound is the first recommended imaging modality in the current guidelines for hernia surgery. 2,3 Advantages of ultrasound are its dynamic character without ionizing radiation, the ability to directly correlate physical examination to imaging findings and relatively low costs. ${ }^{4-6}$ Disadvantages are intra-observer variety and intra-observer accuracy. ${ }^{7}$

When a patient presents with groin complaints in primary care, ultrasound is frequently requested before referring to a surgical specialist. Given that an inguinal hernia can be easily diagnosed clinically in the greatest part of patients, it is questionable whether all requested imaging would be necessary to obtain the correct diagnosis and to decide whether patients should be referred for possible surgical treatment.

The aim of this study was to evaluate the utilization and influence of groin ultrasound requested by the general practitioner (GP) in patients with (suspicion of) an inguinal hernia referred to the surgical outpatient department.

\section{METHODS}

A retrospective observational analysis using a prospectively maintained database was performed in a hernia clinic specialized in endoscopic totally extraperitoneal (TEP) hernia repair in the Netherlands.

All adult patients referred to the surgical outpatient department between July 2016 and July 2017 who underwent groin ultrasound for suspicion of inguinal hernia upon request of the GP in advance were identified. Performance of ultrasound was not restricted to a particular radiologist or this hernia clinic. As long as the ultrasound outcome was documented in the referral letter or radiology report the patient was eligible for analysis. Patients referred by other specialists or with an ultrasound requested by any specialist other than the GP, were excluded.

Patient characteristics (age, sex, body mass index (BMI) and American Society of Anesthesiologists (ASA) classification), outcomes of clinical assessment, ultrasound outcomes and, if applicable, intraoperative findings were registered. Outcomes of history and physical examination were divided into positive, negative or inconclusive" for inguinal hernia. In case of 
a reported observable swelling or a palpable mass during the Valsalva manoeuvre, groins were scored positive. In case of incomplete or unspecified documentation, clinical assessment was still taken into account and scored positive, negative, or inconclusive but with the distinction that this could be based on the documented conclusion solely. Ultrasound outcomes were divided into positive or negative for inguinal hernia, based on the referral letter documenting the ultrasound outcome or the radiology report.

Evaluation of groin ultrasound was carried out by comparing ultrasound outcomes with clinical assessment and, if applicable, with subsequent perioperative findings of TEP inguinal hernia repair. Perioperative findings were obtained from the operative reports and divided into "positive" or "negative" for inguinal hernia. In case of discrepant findings between ultrasound and clinical assessment, the influence of ultrasound and physical examination on decisionmaking of the surgeon was compared.

\section{RESULTS}

During the study period, 1371 patients were referred to the surgical outpatient clinic for suspicion of an inguinal hernia. A total of 361 patients (26\%) underwent ultrasound before visiting the surgical outpatient department, involving 399 groins (Table 1). Eighty-six percent of patients were male and $14 \%$ were female. The median age of the study population was 55 years (interquartile range (IQR) 43 - 66 years).

As regards ultrasound outcomes, 374 groins (94\%) were scored positive for an inguinal hernia and 25 groins were scored negative (6\%) (Figure 1). Upon physical examination by the surgeon 316 groins (79\%) were reported positive for an inguinal hernia; in 264 of these cases (84\%) an observable swelling or a palpable mass during the Valsalva manoeuvre was reported, representing $66 \%$ of all 399 groins. In 52 groins reported positive for inguinal hernia on clinical assessment (16\%) solely the surgical conclusion in the medical record was positive. A total of 69 groins (17\%) were scored negative for an inguinal hernia on clinical assessment and in 14 groins (4\%) the surgeon doubted if an inguinal hernia was present.

A discrepancy between outcomes of ultrasound and physical examination was present in 58 groins (15\%) of which 21 groins underwent inguinal hernia surgery (36\%). In seven groins with discrepant findings (12\%) physical examination was positive, contrary to a negative ultrasound. All seven groins underwent hernia repair based on physical examination and an inguinal hernia was found perioperatively in all cases. In 51 groins with discrepant findings (88\%) ultrasound was positive, contrary to a negative physical examination. Fourteen of these groins (27\%) underwent hernia repair and an inguinal hernia was found perioperatively in 12 cases (86\%). In the remaining 37 groins, an expectative policy was applied based on physical examination. In fourteen groins (4\%) ultrasound was positive, but findings upon physical examination were inconclusive. Nine of these groins underwent hernia repair and an inguinal hernia was found perioperatively in all groins. 
Sex, $\mathrm{n}(\%)$

Male

$312(86.4)$

Female

$49(13.6)$

Age (years)

$55.0(43.0-65.5)$

BMI $\left(\mathrm{kg} / \mathrm{m}^{2}\right)$

ASA-classification, $\mathrm{n}(\%)$

I

$187(51.8)$

II

$141(39.0)$

III

$32(8.9)$

IV

Ultrasound , $\mathrm{n}(\%)$

Unilateral

Bilateral

Location of alleged hernia per groin, $n(\%)$

Left

Right

$225(56.4)$

Continuous data are presented as median (Interquartile range) BMI: Body Mass Index, ASA: American Society of Anesthesiologists

Figure 1. Interrelation between ultrasound and physical examination and number of cases in which was proceeded to hernia repair $(n=399)$

\begin{tabular}{|c|c|c|}
\hline & Ultrasound + & Ultrasound - \\
\hline Physical examination + & $309(77.4)$ & $7(1.7)$ \\
Hernia Repair & $272(88)$ & $7(100)$ \\
\hline Physical examination - & $51(12.8)$ & $18(4.5)$ \\
Hernia Repair & $14(27.5)$ & $3(16.7)$ \\
\hline Physical examination $+/-$ & $14(3.5)$ & - \\
\hline Hernia Repair & $9(64.3)$ & \\
\hline
\end{tabular}

Values are reported as $n(\%)+$ : positive, -: negative, $+/-$ : inconclusive 
In 18 groins both ultrasound and physical examination were scored negative. Three of these groins were operated due to a strong wish of the patients. In one patient an inguinal hernia was found perioperatively.

\section{DISCUSSION}

Even though inguinal hernia is a predominantly clinical diagnosis, this study demonstrates that approximately one quarter of newly referred patients with suspicion of an inguinal hernia to the surgical outpatient department of a Dutch hernia clinic underwent ultrasound in advance. In nearly $80 \%$ of these patients a clinically apparent inguinal hernia was found upon surgical assessment, implying that in this subset of patients ultrasound may have been unnecessarily performed. These results point out that ultrasound diagnostics in patients with clinically apparent inguinal hernias are redundantly requested in primary care.

Ultrasound and physical examination conducted by the surgeon yielded discrepant results in $15 \%$ of cases, in which surgical treatment was based on interpretation of clinical findings in $76 \%$ of cases and based on interpretation of ultrasound findings in only $24 \%$. All patients in whom ultrasound was negative yet physical examination positive underwent surgical treatment. In all cases a hernia was found intraoperatively, confirming a high accuracy of physical examination. Of patients with a negative clinical examination yet positive ultrasound only $27 \%$ underwent surgery, which demonstrates that a positive ultrasound finding alone is in the majority of cases not sufficient for proceeding to surgery. In $86 \%$ of operated cases in which physical examination was negative yet ultrasound positive for an inguinal hernia, a hernia was found intraoperatively. Even though in the great majority of these patients the hernia was confirmed during surgery, it is important to realize that ultrasound does not accurately predict the presence of an inguinal hernia in all cases.

A study performed by Kim et al. investigated the utilisation of ultrasound for suspicion of inguinal hernias as well. ${ }^{8}$ This study evaluated all ultrasound examinations, either requested through primary or secondary care, performed in patients seen at the surgical outpatient department for clinical suspicion of an inguinal hernia. In this study, 267 ultrasounds were conducted of which 105 (39\%) were positive for an inguinal hernia on physical examination by the surgeon. Although this percentage is considerably lower than the $66-79 \%$ described in this study, it still represents significant overuse of diagnostic ultrasound and supports the results of this study. A possible explanation for the lower numbers of both positive physical examination and positive ultrasound findings in this study may be that approximately two third of ultrasound examinations were requested by the surgeon. Logically, a lower number of clinically evident hernias seen by the surgeon in this study would then be expected.

The study by Kim et al. showed discrepant findings between physical examination and ultrasound in $30 \%$ of the population. ${ }^{8}$ In this study, however, one proceeded to surgery in only $13 \%$ of cases in which ultrasound was negative and physical examination positive. In case of positive ultrasound and negative clinical examination 30\% of patients were operated. Findings of the studies performed by Light et al. and Bradley et al. ,that examined the accuracy of ultrasound in diagnosing clinically inapparent inguinal hernias, yielded higher percentages 
of surgical treatment in patients with negative clinical findings and positive ultrasound, of respectively $70 \%$ and $95 \%$. 9,10

To our knowledge, this is the first study focusing solely on ultrasound for suspicion of inguinal hernia requested in primary care. Since all consecutive 1371 patients that newly presented at the surgical outpatient department of a hernia clinic within a year were screened and analyzed, we think the numbers reported in our study provide an accurate representation of the general patient population with inguinal complaints at the surgical outpatient clinic.

Due to its retrospective character, this study has some limitations. Physical examinations were not extensively reported in all cases and must therefore be interpreted with some caution. In 52 cases with clinical findings reported positive for an inguinal hernia (16\%), only the conclusion was noted as such but the actual findings upon physical examination (clinically detectable bulge, Valsalva manoeuvre) were not reported. In these cases we cannot be as sure of a truly clinically detectable inguinal hernia as in the extensively documented cases. Another important consideration for the groins positively scored for an inguinal hernia on clinical assessment, is that there might be a possibility that the surgeon was influenced by the ultrasound results if they were already reported in the referral letter before the patient was clinically assessed. Also, it does not become entirely clear in all patients how decisions regarding proceeding or not proceeding to operative repair were made. Apart from ultrasound and physical examination, other reasons (eg. strong wish of the patient) could not be extracted in all cases.

Another limitation of this study is that intra-observer variety and intra-observer accuracy between different radiologists might have played a role. Although the inclusion of every ultrasound, regardless of where it was performed, reflects daily clinical practice, the lack of an uniform judgment of ultrasound examinations might not make their results fully comparable.

Lastly, no information could be obtained about the patients in whom the GP requested an ultrasound for suspicion of inguinal hernia that were not referred to the surgical outpatient department, since ultrasound was negative. Additional information about the number of cases in which performing ultrasound prevented unnecessary referral to the surgeon and costs would have provided even more insight.

There may be several explanations why ultrasound diagnostics for suspicion of inguinal hernias are frequently requested in primary care. Firstly, not all GPs may be capable of clinically diagnosing an inguinal hernia and may ask for an ultrasound in case of any inguinal complaints relatively soon. A second consideration may be that when GPs do suspect an inguinal hernia, US is requested for confirmation of their clinical suspicion before referral to the surgeon. Also, GPs might be scared of patients developing an incarcerated hernia might they have missed the diagnosis of an inguinal hernia previously, and therefore perform ultrasound to confirm or exclude the diagnosis of an inguinal hernia. However, the risk of an inguinal hernia becoming incarcerated is as low as less than $3 \%$ per year. ${ }^{2}$ In the female population, clinically diagnosing an inguinal hernia is presumably even more difficult for the GP, most likely caused by the relatively high numbers of femoral hernias in women. This assumption is confirmed by the finding of a relatively high percentage of women that underwent ultrasound through primary care in this study. 
The current total healthcare costs are high and expanding, and much effort is invested to control increasing costs. ${ }^{11}$ This warrants careful evaluation of the utilisation and cost aspects of health care resources. Since radiology departments often enable GPs to refer patients for ultrasound examination directly without prior referral to secondary care, it is important that GPs become aware of the current groin ultrasound overdiagnostics and their accompanying costs.

However, as not all inguinal hernias are clinically apparent and a certain difference in experience and clinical skills in diagnosing inguinal hernias between GPs and surgeons can be expected, it remains logical that GPs request groin ultrasound in particular cases. Moreover, ultrasound diagnostics are expected to be cheaper and more efficient compared to surgical referral of all unclear or doubtful cases, since most patients with negative findings for inguinal hernia on groin ultrasound, which is expected to cost less than surgical referall, will not be seen by the surgeon and costs and time will be saved.

Even though a difference in expertise between the GP and the surgeon exists, it is of high importance GPs are adequately schooled and instructed in performing adequate physical examination for the diagnosis of inguinal hernia, the clinical signs of an inguinal hernia and the indications for additional imaging. We recommend performing physical examination with the patient in standing position, in which it is likely that the swelling can already be seen in most cases. Only when doubt exists after inspection and performance of the Valsalva manoeuvre in this position, palpation of the inguinal canal is necessary. In case no swelling can be seen or palpated, an incarcerated hernia can reliably be excluded. In case the GP has a reasonable suspicion of an inguinal hernia after physical examination, direct surgical referral is advisable. With regard to performance of additional imaging, it is important GPs are aware that imaging is not necessary for diagnosis confirmation when a clinically apparent hernia is present, and that in case of abcense of or doubt about clinical signs of an inguinal hernia the ultrasound diagnosis of an inguinal hernia may not always be correct or related to the complaints the patient is experiencing. 


\section{REFERENCES}

1. Van den Berg JC, de Valois JC, Go PM, et al. Detection of groin hernia with physical examination, ultrasound, and MRI compared with laparoscopic findings. Investig Radio/ 1999;34(12):739-43.

2. The Herniasurge Group. International guidelines for groin hernia management. Hernia 2018;22:1-165.

3. Simons MP, Aufenacker T, Bay-Nielsen M et al. European Hernia Society guidelines on the treatment of inguinal hernia in adult patients. Hernia 2009;13:343-403.

4. Depasquale R, Landes C, Doyle G. Audit of ultrasound and decision to operate in groin pain of unknown aetiology with ultrasound technique explained. Clin Radio/ 2009;64(6):608-14.

5. Bradley M, Morgan D, Pentlow B et al. The groin hernia - an ultrasound diagnosis? Ann R Coll Surg Engl 2003;85:178-80.

6. Jacobson JA, Khoury V, Brandon CJ. Ultrasound of the groin: techniques, pathology, and pitfalls. AJR Am J Roentgenol 2015;205(3):513-23.

7. Alam A, Nice C, Uberoi R. The accuracy of ultrasound in the diagnosis of clinically occult groin hernias in adults. Eur Radio/ 2005;15(12):2457-61.

8. Kim B, Robinson P, Modi $\mathrm{H}$ et al. Evaluation of the usage and influence of groin ultrasound in primary and secondary healthcare settings. Hernia 2015;19:367-71.

9. Light D, Ratnasingham K, Banerjee A et al. The role of ultrasound scan in the diagnosis of occult inguinal hernias. Int J Surg 2011;9(2):169-72.

10. Bradley M, Morgan J, Pentlow B et al. The positive predictive value of diagnostic ultrasound for occult herniae. Ann R Coll Surg Eng/ 2006;88(2):165-7.

11. Koning GG, Adang EM, Stalmeier PF et al. TIPP and Lichtenstein modalities for inguinal hernia repair: a cost minimisation analysis alongside a randomised trial. Eur J Health Econ 2013;14(6):1027-34. 


\title{
Incidental inguinal hernia in men
}

\author{
CV van Hessen \\ WJ Bakker \\ FBM Sanders \\ EJMM Verleisdonk \\ GJ Clevers \\ PHP Davids \\ M Avci \\ JPJ Burgmans
}

Submitted 


\section{ABSTRACT}

\section{Background}

Ultrasound may contribute to establish the cause of nonspecific groin complaints. However, the risk is diagnosing an incidental inguinal hernia whereas the pain has an alternative cause. Overtreatment is to be prevented. Therefore, the aim of this study is to determine the prevalence of a previously unknown inguinal hernia among working-age men without groin complaints.

\section{Methods}

A cross sectional study was conducted in healthy men aged 45-67 years. Men with a Body Mass Index $(\mathrm{BMI})>40$, a history of groin complaints, a known inguinal hernia or previous inguinal surgery were excluded. Ultrasound of both groins was performed in supine position with and without a Valsalva manoeuvre by a specialised ultrasound technician in consultation with a radiologist. In all groin ultrasounds showing an inguinal hernia, physical examination was executed by a hernia surgeon.

\section{Results}

In the months June and November of 2018, 200 groins of 100 men were analysed. In 16 (16\%) men an inguinal hernia was found on groin ultrasound (95\% confidence interval [8.8 - 23.2]). In 12 men this was a unilateral inguinal hernia and in 4 men a bilateral inguinal hernia. Ultrasound yielded no other pathology.

\section{Conclusion}

In a population of men aged 40-67 years without groin complaints, ultrasound detects an inguinal hernia in $16 \%$. Hence, the probability of wrongly attributing groin complaints to an incidental inguinal hernia, diagnosed on ultrasound, is considerable. 


\section{BACKGROUND}

An inguinal hernia is one of the most common surgical diagnose worldwide and its repair accounts for an important part of health care expenditure. The lifetime risk of developing a groin hernia in men has been estimated to be $27 \%{ }^{1}$

A symptomatic clinical apparent inguinal hernia is a well-known condition and the appropriate operative or non-operative management is straightforward in the majority of cases. ${ }^{2}$ However, groin complaints in patients without an apparent inguinal hernia remain a clinical challenge. ${ }^{3,4}$ Despite the broad differential diagnosis of groin pain, an inguinal hernia is often suspected as the cause of complaints. To confirm a suspected inguinal hernia, ultrasound is widely used and a hernia is often suggested. Although ultrasound certainly has a place in the work-up of nonspecific groin complaints, it is questionable whether inguinal hernias solely diagnosed on ultrasound always explain the (nonspecific) groin pain. A recent study suggest that occult inguinal hernias (the presence of a groin hernia on ultrasound that could not be diagnosed clinically) are more likely to develop chronic postoperative pain, thus the suspicion of the existence of an alternative cause for the pre-operative pain is plausible. ${ }^{5}$

Moreover, in case of ambivalent clinical findings, both the sensitivity and specificity of groin ultrasound cannot be reliably determined based on current evidence. Although the positive predictive value of groin ultrasound is rather high, it is not $100 \%$, which makes it possible that the inguinal hernia doesn't truly exists. ${ }^{6}$ Finally, as approximately one-third of inguinal hernia patients are asymptomatic ${ }^{7}$, the question remains if the hernia on additional imaging could be an incidental finding that does not cause pain. Hence, a result of performing groin ultrasound in patients with pain and no clinical apparent hernia, could be diagnosing and unnecessary operating inguinal hernias that would not have caused clinical consequences during a patient's lifetime if left untreated.

All published data on groin ultrasound concerned clinically evident inguinal hernias or symptomatic clinically occult hernias. Studies on the prevalence of inguinal hernias as an incidental finding on ultrasound have never been published. The aim of this study was to determine the prevalence of a previously unknown inguinal hernia among healthy, workingage men without groin complaints.

\section{METHODS}

A cross-sectional study was performed in a Dutch general hospital. Healthy men (ASA I or II)

between 40 and 67 years old (based on the mean age of an inguinal hernia detection +/- 1 standard deviation of patients in a large study previously conducted in this Dutch hospital ${ }^{8}$ ), with a Body Mass Index (BMI) of under 40 and without groin complaints or a medical history of an inguinal hernia were recruited from the plaster room and trauma outpatient clinic. After informed consent was received, a groin ultrasound was performed of both groins in supine position with and without a Valsalva manoeuvre. All examinations were carried out by one 
specialised ultrasound technician. Only in case of doubt or unexpected findings, a specialised radiologist was consulted. An inguinal hernia was defined as a protruding bulge $>0.8 \mathrm{~cm}$ through the abdominal wall, lateral or medial to the epigastric vessels, irrespective of the size of the defect (gap size). Similar appearances in the femoral canal were classified as femoral hernia. Statistical analyses were performed using SPSS statistical software, version 24 (IBM Corp, Armonk, NY). Descriptive statistics were used for baseline data.

\section{RESULTS}

In the months June and November of 2018, 100 men were enrolled in the study and 200 groins examined. The median age of the study population was 53 years (interquartile range (IQR) 47- 61 years) and the median BMI was $26.6 \mathrm{~kg} / \mathrm{m} 2$ (IQR 23-30). Baseline characteristics are shown in table 1.

Table 1. Patient characteristics ( $n=100,200$ groins)
Age (years),
$53.0(47.0-61)$
BMI $\left(\mathrm{kg} / \mathrm{m}^{2}\right)$,
$26.6(23-30)$

\section{Smoking, $\mathrm{n}(\%)$}

Current smoker

Non-current smoker

Legend. Continous data are presented as median (interquartile range). BMI: body mass index.

Among the 100 included participants, in 16 an inguinal hernia was found with groin ultrasound (proportion = 16\%; 95\% confidence interval (Cl), 8.8-23.2). In 12 men this concerned a unilateral inguinal hernia, and in 4 men a bilateral inguinal hernia. Both physical examination and ultrasound yielded no other pathology.

\section{DISCUSSION}

In $16 \%$ of men between 40 and 67 years old without groin complaints and without a medical history of an inguinal hernia or groin surgery, an inguinal hernia is found on groin ultrasound. This high prevalence, along with the widespread use of diagnostic groin ultrasound, suggests a substantial risk for incidentally detected inguinal hernias that may wrongly be considered as the cause of groin pain. This might lead to overtreatment and unnecessary hernia repair. To 
our knowledge this is the first study assessing the prevalence of inguinal hernias on ultrasound among asymptomatic healthy men without groin complaints.

The prevalence of asymptomatic and clinically undetectable inguinal hernias during laparoscopic procedures has been studied before. Watson et al. concluded that these inguinal hernias were common in their surgical population, especially in men (19\%). ${ }^{9}$ However, part of the initial operations (10\%) was performed to repair a (contralateral) inguinal hernia, which makes it less surprising to find an inguinal hernia on the other side as well. More recently, Paajanen et al. found inguinal hernias during laparoscopic operation in 35\% when looking only at men. ${ }^{10}$ However, they did not elaborate on their population other than age and sex. Hence we do not know whether the included patients had a history of groin complaints or hernia repair. Furthermore, their definition of an inguinal hernia was not defined and ambiguous. Every small dimple and minor fascial defect or asymmetrical notch apparently was recorded as an inguinal hernia (e.g. their attached example of a defect of $0.5 \mathrm{~cm}$ ). It is very likely that these small fascial defects are in fact asymptomatic patent processi vaginales (PPV). Van Wessem et al determined a PPV prevalence of $21 \%$ among male patients undergoing abdominal laparoscopy for various pathologies." However, the likelihood of these PPV's becoming symptomatic seems small, considering that autopsy studies show that $15-30 \%$ of adult males without clinically apparent inguinal hernias have patent processi vaginales at death. ${ }^{12,13}$

Compared with the previous studies, the prevalence of incidental inguinal hernias in our study population is slightly lower. This is possibly due to the fact that ultrasonography is not sensitive enough to detect small PPV's. However, the prevalence is still substantial. This may not seem odd if one keeps in mind that the life time risk of developing a groin hernia in men is estimated to be $27 \%$. This percentage reflects the cumulative lifetime risk for inguinal hernia repair from various ages. At birth, the risk of ever developing an inguinal hernia that will have to be repaired is $27.2 \%$, which decreases throughout a man's life. At the age of $45,6.1 \%$ already developed an inguinal hernia and the risk of developing one in the future remains $21.1 \%$. Between the age of 45 and 65 , another $7.5 \%$ will develop an inguinal hernia and at the age of 65 there is still a risk of $13.6 \%$. If we compare our results to this study and we try to speculate on what will happen to the $16 \%$ inguinal hernias we found on Ultrasound, we can roughly divide them into three categories. There will be (a small percentage of) false positive inguinal hernias in which case there is no actual inguinal hernia. There will be inguinal hernias that will never become symptomatic and there will be inguinal hernias that will become symptomatic in the future.

In daily practice, patients with groin complaints and a positive groin ultrasound, without matching clinical features are often referred. It is questionable whether inguinal hernias solely diagnosed on ultrasound always explain the (nonspecific) groin pain, especially since approximately one-third of inguinal hernia patients are asymptomatic and the majority of the remaining two-third experience only mild complaints ${ }^{14,15}$. The therapeutic dilemma of an occult hernia is comparable to the situation of patients with asymptomatic gallstones. After the introduction of ultrasound and its frequent use in patients with vague abdominal complaints, many silent gallstones have been found. ${ }^{16}$ Initially, in most patients cholecystectomy was performed. Later, this aggressive policy was changed to a more conservative approach, since several studies showed only minor differences in outcomes (biliary pain, complications, mortality, etc.) between prophylactic cholecystectomy and an wait and see policy. ${ }^{17}$ However, 
despite the recommendation of not treating incidental gallstones in accordance with the guidelines, the possibility remains that vague abdominal complaints are wrongly attributed to incidental gallstones diagnosed on ultrasound. ${ }^{18,19}$

The clinical relevance of a small inguinal hernia on groin ultrasound is put into question even more by the different ultrasonographic definitions of an inguinal hernia that are being used. In fact, there are no well-defined ultrasonographic criteria to define an inguinal hernia and the diagnosis is often based upon any protrusion through the abdominal wall, irrespective of the size of the protruding bulge and the size of the inguinal defect. Hence, it is not uncommon to receive referrals for a positive groin ultrasound based on a hernia defect size of several millimetres or based on minimal movement of fatty tissue. In this study a protrusion of $>8 \mathrm{~mm}$ was defined as a groin hernia, based on the experience of the radiologist in our hernia centre. However, this is not a foregone conclusion.

We conclude that incidental inguinal hernias diagnosed with ultrasound are common. The probability that groin complaints are wrongly attributed to an inguinal hernia, appears to be considerable. Both general practitioners and hernia surgeons should be aware of the high prevalence of incidental groin hernias on ultrasound and should show restraint when it comes to imaging the groin region in case of clinically unapparent hernias. In the work-up of groin complaints it is important to realise the broad differential diagnosis and first rule out other causes of groin pain.

\section{REFERENCES}

1. Kingsnorth A, LeBlanc K. Hernias: Inguinal and incisional. Lancet. 2003;362:1561-1571. doi:http://dx.doi. org/10.1016/SO140-6736\%2803\%2914746-O

2. The Herniasurge Group (2018) International guidelines for groin hernia management. Hernia 22:1-165.

3. Fitzgibbons RJ, Giobbie-Hurder A, Gibbs JO, et al. Watchful waiting vs repair of inguinal hernia in minimally symptomatic men: a randomized clinical trial. JAMA. 2006;295(3):285-292. doi:10.1001/ jama.295.3.285

4. O'Dwyer PJ, Norrie J, Alani A, Walker A, Duffy F, Horgan P. Observation or operation for patients with an asymptomatic inguinal hernia: a randomized clinical trial. Ann Surg. 2006;244(2):167-173. doi:10.1097/01. sla.0000217637.69699.ef.

5. L. van Hout, W. J. V. Bökkerink, M. S. Ibelings, J. Heisterkamp, P. W. H. E. Vriens (2018) Outcomes of surgery on patients with a clinically inapparent inguinal hernia as diagnosed by ultrasonography. Hernia (2018) 22:525-531

6. Kwee RM, Kwee TC (2018) Ultrasonography in diagnosing clinically occult groin hernia: systematic review 
and meta-analysis. Eur Radio/ 28(11):4550-4560.

7. van den Berg JC, de Valois JC, Go PM, Rosenbusch G. Detection of groin hernia with physical examination, ultrasound, and MRI compared with laparoscopic findings. Invest Radiol. 1999;34(12):739743.

8. Roos MM, Bakker WJ, Schouten N, Voorbrood CEH, Clevers GJ, Verleisdonk EJMM, Davids PH, Burgmans JPJ (2018) higher recurrence rate after endoscopic totally extraperitoneal (TEP) inguinal hernia repair with Ultrapro lightweight mesh: 5-year results of a randomized controlled trial (TULP-trial). Ann Surg 2018; 268(2):241-6

9. D.S. Watson, K.W. Sharp, J.M. Vasquez, W.O. RichardsIncidence of inguinal hernias during laparoscopy South Med J, 87 (1994), pp. 23-25

10. Paajanen H, Ojala S, Virkkunen A. Incidence of occult inguinal and Spigelian hernias during laparoscopy of other reasons. Surgery. 2006;140:9-12; discussion 12-13

11. van Wessem KJP, Simons MP, Plaisier PW, Lange JF. The etiology of indirect inguinal hernias: congenital and/or acquired? Hernia. 2003;7(2):76-79.

12. Brendan Devlin H, Kingsnorth A, O’Dwyer PJ, Bloor K (1998) Epidemiology and Aetiology of Primary Groin Hernias in Adults. In: Management of Abdominal Hernias, 2nd edn. : Chapman \& Hall Medical, London, Chap 3

13. Abrahamson J (1998) Etiology and Pathophysiology of Primary and Recurrent Groin Hernia Formation. Surg Clin N Am 78(6):953-972

14. van den Berg JC, de Valois JC, Go PM, Rosenbusch G. Detection of groin hernia with physical examination, ultrasound, and MRI compared with laparoscopic findings. Invest Radiol. 1999;34(12):739743.

15. Burgmans JPJ, Schouten N, Clevers GJ, Verleisdonk EJMM, Davids PHP, Voorbrood CEH, Simmermacher RKJ, van Dalen T (2015) Pain after totally extraperitoneal (TEP) hernia repair might fade out within a year. Hernia. 2015;19(4):579-585. doi: 10.1007/s10029-015-1384-3.

16. Peter P, Zeller A, Sonnenberg A. (1985) Incidence of asymptomatic gallstones in hospital patient S Z Gastroenterol. 23(10):573-8. 
17. Ransohoff DF, Gracie WA, Wolfenson LB, Neuhauser D. (1983) Prophylactic cholecystectomy or expectant management for silent gallstones. A decision analysis to assess survival. Ann Intern Med. 1983 Aug;99(2):199-204.

18. Fendrick AM', Gleeson SP, Cabana MD, Schwartz JS (1993). Asymptomatic gallstones revisited. Is there a role for laparoscopic cholecystectomy? Arch Fam Med. 2(9):959-68.

19. Anu Behari, V. K. Kapoor (2012) Asymptomatic Gallstones (AsGS) - To Treat or Not to? Indian J Surg. 74(1): 4-12.] 


\title{
CHAPTER 5
}

\section{An 11-year analysis of reoperated groins after endoscopic totally extraperitoneal (TEP) inguinal hernia repair in a high volume hernia center}

\author{
MM Roos \\ CV van Hessen \\ EJMM Verleisdonk \\ GJ Clevers \\ PHP Davids \\ CEH Voorbrood \\ RKJ Simmermacher \\ JPJ Burgmans
}




\section{ABSTRACT}

\section{Background}

Developments in inguinal hernia surgery have substantially lowered recurrence rates, yet recurrences remain an important outcome parameter of inguinal hernia repair. The aim of this study was to analyze the characteristics of all reoperated groins after endoscopic totally extraperitoneal (TEP) inguinal hernia repair in a high-volume hernia clinic in the Netherlands.

\section{Methods}

All groins with recurrence-like symptoms reoperated after previous TEP inguinal hernia repair between January 2006 and December 2016 were analyzed. Patient characteristics, imaging findings, primary hernia type, time to recurrence and recurrence type were assessed.

\section{Results}

A total of 137 groins were reoperated in 130 patients. The median age at the TEP procedure was 54 years (interquartile range (IQR) 45-64 years). Fifty-seven groins were initially part of a bilateral procedure (42\%). Median time until recurrence was 9 months (IQR 4-26 months). Reoperation findings were a hernia recurrence in $76 \%$, an isolated lipoma in $18 \%$, and no recurrence or lipoma in $6 \%$. The majority of hernias recurred at their initial site (70\%), of which the greatest part involved direct hernias. Isolated lipomas were more frequently seen after indirect hernia repair.

\section{Conclusion}

Inguinal hernia recurrences were still observed in this high-volume hernia clinic. Recurrences were most frequently seen at their initial hernia site, the majority involving direct hernias. Isolated lipomas presenting as a pseudorecurrence were most frequently seen after correction of indirect hernias. In accordance with the current guidelines, reducing recurrence rates can be achieved by mesh fixation in bilateral, large and direct defects and by thoroughly reducing lipomas. 


\section{BACKGROUND}

Once inguinal hernia repair was accompanied by a substantial number of recurrences, yet recurrence rates decreased tremendously since mesh repair has become routine.-4 Consequently, follow-up after inguinal hernia repair focused less on recurrence and shifted towards postoperative pain. The posterior approach of the inguinal area with a preperitoneal position of mesh demonstrated a significant reduction in chronic pain compared to an anterior approach. ${ }^{5}$ Therefore, the endoscopic totally extraperitoneal (TEP) inguinal hernia repair is widely used and by many surgeons considered the preferable preperitoneal technique. ${ }^{6-11}$

Conceptually, a retromuscular and preperitoneal position of mesh covering Fruchaud's triangle with overlap, should eliminate the risk of recurrence. However, recurrences after TEP inguinal hernia repair still develop and remain a clinical problem and an important outcome parameter of inguinal hernia surgery. ${ }^{12-16}$

It has been demonstrated that surgical skills and experience contribute to the recurrence risk after endoscopic inguinal hernia repair. Low long-term recurrence rates have been achieved in highly specialized centers, implying that volume may have a positive impact on recurrence rates. ${ }^{17-20}$

Concerning the TEP technique in particular, recurrences have been associated with the learning curve of the individual surgeon. . $^{-11,21,22}$

As we are still confronted with recurrences after primary TEP inguinal hernia repair, analyzing operative outcomes of recurrent hernias in a high-volume center specialized in TEP hernia repair with low recurrence rates may facilitate better understanding of their cause and may contribute to detecting patterns of recurrence and possible prevention of recurrence development. The objective of this study was to provide an 11-year analysis of all reoperated groins for recurrence-like symptoms in a high-volume hernia center in the Netherlands specialized in TEP inguinal hernia repair.

\section{METHODS}

\section{Study design}

The characteristics of all groins with recurrence-like complaints that were reoperated between 2006 and 2017 in our hernia clinic were studied retrospectively.

During the study period, approximately 1000 patients were operated annually by TEP inguinal hernia repair by five experienced surgeons with extensive experience in this technique ( $>500$ procedures per surgeon). Two- and five-year recurrence rates in this center are respectively $0.8 \%$ and $1.1 \% .19,20$

Institutional Review Board approval was obtained for this study. 


\section{Patients}

All patients reoperated for recurrence-like complaints between January 1, 2006 and December 31, 2016 after previous unilateral or bilateral TEP hernia repair in this hernia clinic were identified and screened for eligibility. All patients had undergone endoscopic TEP hernia repair under general anaesthesia in a day-case setting. Operative details of this technique have been described previously. ${ }^{14}$ In all subjects a synthetic mesh (Prolene ${ }^{\circledR}$ or Ultrapro ${ }^{\circledR}, 10 \times 15$ $\mathrm{cm}$ ) was placed preperitoneally over the hernia defect. Prolene ${ }^{\oplus}$ is the mesh standardly used in this center, but from 2010 until 2012 Prolene $^{\circledR}$ mesh was compared to Ultrapro ${ }^{\circledR}$ mesh in a randomized controlled trial..$^{19,20}$ Mesh fixation was not routinely performed.

For evaluation of recurrence-like complaints after TEP repair, patients presented upon own initiative, through referral by their general practitioner or for follow-up regarding a randomized controlled trial conducted in this center. ${ }^{19,20}$ Evaluation at the surgical outpatient department consisted of history taking, physical examination and, when deemed necessary, additional imaging by ultrasonography, MRI or CT. A recurrence was assumed in case of a typical history of an inguinal swelling, a clinically detectable bulge in the abdominal wall exacerbated by the Valsalva manoeuvre, or suspicion of a hernia recurrence on additional imaging. Decisions to reoperate were based on a clinical or radiological suspicion of a recurrent hernia, a clinical or radiological suspicion of an isolated lipoma assumed to cause the recurrence-like complaints, or in case of a strong wish of the patient. Clinical suspicion of a recurrence when radiological findings were negative for a recurrence was also an indication for reoperation. The following exclusion criteria were applied: Patients who were referred after TEP performed elsewhere, patients who presented with recurrence-like symptoms who underwent another type of hernia repair after the initial TEP or patients who were operated for suspicion of recurrence elsewhere.

\section{Outcomes}

Patient characteristics (age, sex, body mass index (BMI)), imaging findings and time to recurrence after TEP repair were registered. Imaging findings (ultrasound, MRI or CT) were divided into recurrence, no recurrence or an isolated lipoma. The date when a patient visited the outpatient clinic with recurrence-like complaints was used as a surrogate for date of recurrence. Time to recurrence was defined as the interval between the TEP procedure and the day of re-attendance at the outpatient clinic.

The following peroperative findings of initial TEP repairs and subsequent operations for recurrences were assessed: Operation date, type of procedure (only for reoperations), side (left/right/bilateral), presence of hernia, hernia type, presence of lipoma, type of mesh, fixation of mesh and duration of the operation. When assessing the type of recurrence upon the second operation the following distinction was made: A protrusion through a weakness in the posterior wall of the inguinal canal (fascia transversalis) was classified as a direct recurrence, a protrusion through the deep inguinal ring (hernia sac or fatty tissue) was classified as an indirect recurrence, and solely fatty tissue not originating from the deep inguinal ring was classified as an isolated lipoma. The size of the hernia defect was not standardly reported in 
operation charts, but large initial defects were scored when reported as such by the operating surgeon.

Since several patients were bilaterally operated for recurrence, recurrence characteristics are presented for groins instead of patients.

\section{Analysis}

Statistical analyses were performed using SPSS version 23.0 (IBM Corp., Armonk, NY, USA).

The cumulative percentage of recurrences was assessed by Kaplan Meier analysis.

For comparison of categorical variables, two-sided p-values were calculated from Pearson's chi-square and Fisher's exact test. For comparison of continuous (non-parametric) variables the Mann-Whitney $U$ test was used.

\section{RESULTS}

Between January 2006 and December 2016, 130 patients were seen at the outpatient clinic and reoperated for suspicion of 137 recurrent hernias after TEP hernia repair (Table 1). The majority of patients were male (96\%) and the median age at the initial TEP procedure was 54.5 years (interquartile range (IQR) 44.8-63.6 years).

Table 1. Patient characteristics $(n=130)$

Sex, n $(\%)$
Male

Female

BMI $\left(\mathrm{kg} / \mathrm{m}^{2}\right)$

Age at initial TEP procedure (years)

Location of initial hernia, $n$ (\%)

Unilateral

Bilateral

Median time to recurrence* (months)
$125(96)$

5 (4)

$25.2(23.2-27.8)$

$54.5(44.8-63.6)$

80 (62)

50 (38)

$9.4(3.6-25.5)$

Legend. Continuous data are presented as median (Interquartile range) BMI: Body Mass Index, TEP: totally extraperitoneal * Displayed for total number of groins, since 7 patients developed a bilateral recurrence $(n=137$

Primary TEP hernia repairs were performed between October 2001 and March 2016 (Table 2). Fifty-seven (42\%) groins were part of a bilateral procedure. In 12 cases (9\%) the initial TEP repair had been for a recurrent hernia after primary open repair, these cases thus present rerecurrences. 
Table 2. Operative characteristics of groins reoperated for clinical suspicion of recurrence $(n=137)$

Initial TEP procedure, side $\mathrm{n}(\%)$

$\begin{array}{ll}\text { Unilateral, left } & 36(26) \\ \text { Unilateral, right } & 44(32) \\ \text { Bilateral, left } & 25(18) \\ \text { Bilateral, right } & 32(24)\end{array}$

TEP procedure, operation time (minutes)

Unilateral

Bilateral

$31.0(24.0-45.0)$

Reoperation, side $\mathrm{n}(\%)$

Unilateral, left

Unilateral, right

$72(52)$

Bilateral, left

Bilateral, right

\section{Reoperation, type of procedure n (\%)}

Lichtenstein

TEP

Stoppa

Fabricius repair

Repair femoral hernia with preperitoneal mesh

Inguinal exploration, lipoma removal

Inguinal exploration, suturing small femoral defect 1 (1)

Legend. Continuous data are presented as median (Interquartile range). TEP: totally extraperitoneal

The median time to detection of recurrence was 9.4 months (IQR 3.6-25.5 months) with the two earliest detected recurrences four days after surgery and the latest detected recurrence 11 years after surgery. The majority of recurrences were diagnosed in the first two years after surgery. At three months postoperatively, 25 (18\%) recurrences were diagnosed, increasing to 78 recurrences at one year postoperatively (57\%) and 100 recurrences (73\%) at two years postoperatively (Figure 1).

At the TEP procedures 123 Prolene $^{\circledast}$ (90\%) and 14 Ultrapro ${ }^{\circledR}$ (10\%) meshes were used. During the initial procedures 68 direct, 63 indirect, four mixed and two femoral hernias were observed (Table 3). Mesh fixation was performed in seven groins (5\%), six involved bilateral repairs (three direct and three indirect hernias) and one involved a large unilateral direct defect. An additional 10 primary large hernias (three direct and seven indirect defects) were described of which none were fixated.

The majority of patients were reoperated through an open anterior approach according to 
Figure 1. Cumulative percentage of recurrences

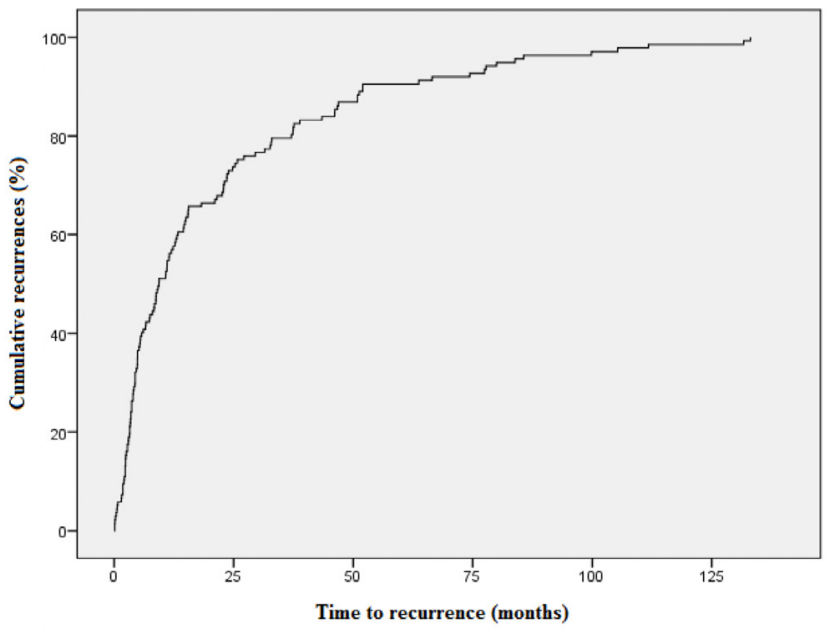

Table 3. Correlation between peroperative findings upon initial TEP repair and reoperation $(\mathrm{n}=137)$

\begin{tabular}{ll}
\hline Primary direct hernia, $\mathrm{n}(\%)$ & $\mathbf{6 8}(\mathbf{5 0})$ \\
Direct recurrence & $46(68)$ \\
Indirect recurrence & $7(10)$ \\
Mixed recurrence (direct and indirect) & $1(1)$ \\
Femoral recurrence & $2(3)$ \\
Recurrence, type unclear & $4(6)$ \\
No recurrence, only lipoma & $4(6)$ \\
No recurrence or lipoma & $4(6)$ \\
Primary indirect hernia, $\mathrm{n}(\%)$ & $\mathbf{6 3}(46)$ \\
Direct recurrence & $15(24)$ \\
Indirect recurrence & $20(32)$ \\
Mixed recurrence (direct and indirect) & $2(3)$ \\
Femoral recurrence & $1(2)$ \\
Recurrence, type unclear & $2(3)$ \\
No recurrence, only lipoma & $19(30)$ \\
No recurrence or lipoma & $4(6)$ \\
Primary mixed hernia, $\mathrm{n}(\%)$ & $\mathbf{4 ( 3 )}$ \\
Direct recurrence & $4(100)$ \\
Primary femoral hernia, $\mathrm{n}(\%)$ & $\mathbf{2 ( 1 )}$ \\
No recurrence, only lipoma & $2(100)$ \\
\hline
\end{tabular}


Lichtenstein (124 groins, 91\%) (Table 2). In three early recurrences (four,four and five days after surgery) two TEP procedures and a Stoppa procedure were executed. In seven patients (5\%) an anterior approach without placement of mesh was performed.

Table 3 demonstrates the correlation between the peroperative findings upon initial TEP repair and the operation for (suspected) recurrence. In all reoperated groins, 104 recurrences were found (76\%) and in 33 cases no hernia could be objectified (24\%). Twenty-five cases yielded an isolated lipoma (18\%) and in eight patients no recurrence or lipoma was found (6\%).

The recurrent hernias described upon reoperation were direct in 65 cases (63\%), indirect in 27 cases (26\%), mixed in three cases (2\%), femoral in three cases (2\%) and in six groins the type of recurrence was not clearly specified (4\%). Seventy-three hernias recurred at their initial site (46 direct to direct, 20 indirect to indirect, four mixed to direct, one direct to mixed and two indirect to mixed), representing $70 \%$ of recurrences and $53 \%$ of all reoperated groins. Initial direct defects recurred significantly more as direct or mixed hernias compared to to indirect defects recurring as indirect or mixed hernias ( $69 \%$ versus $35 \%, p=0.000$ ).

Nine out of eleven primary defects that were reported as large hernias recurred as the same type of hernia (82\%) (four direct hernias, five indirect hernias) and four out of seven primary fixated hernias (57\%) (three direct hernias, one indirect hernia) recurred at their initial site.

In 25 reoperated cases (18\%) an isolated lipoma without a hernia recurrence was found (initially 19 indirect hernias, four direct hernias and two femoral hernias). Significantly more of these lipomas were seen after correction of indirect hernias in comparison to direct hernias (30\% versus $6 \%, p=0.000$ ).

Of the cases where no recurrence or lipoma was found, one patient had developed a hydrocele and in one patient bulging of the abdominal wall was observed. In five patients no abnormalities were found. One early assumed recurrence underwent a second TEP four days postoperatively; no recurrence but only hematoma was found.

When comparing time to recurrence for the true recurrent hernias compared to the findings of an isolated lipoma or no recurrence no significant differences were found (Tab/e 4). Also, no significant difference in time to recurrence could be found for initially unilateral or bilateral hernias and different hernia types.

Imaging was performed in 81 groins before reoperation (59\%). Imaging findings and findings upon reoperation yielded similar results in 58 cases (72\%). In 79 groins ultrasound was conducted; 65 of these ultrasounds were conclusive and diagnosed 56 hernias (peroperatively 41 recurrent hernias, 10 solitary lipomas and no recurrence or lipoma in five cases), three lipomas (peroperatively one recurrent hernia and two lipomas) and no abnormalities in six cases (peroperatively four hernia recurrences and no recurrence or lipoma in two cases). In ten cases ultrasound was inconclusive and followed by MRI; five hernias and five lipomas were diagnosed (peroperatively four hernia recurrences and six lipomas). In four cases ultrasound was inconclusive followed by $\mathrm{CT}$; three hernias were diagnosed (peroperatively two recurrences and no recurrence or lipoma in one case), in one case no abnormalities were 
described (peroperatively a lipoma). In one case only CT was performed and a hernia was diagnosed (peroperatively a recurrent hernia) and in one case only MRI was performed and a lipoma was diagnosed (peroperatively a lipoma).

Table 4. Comparison of time to clinical diagnosis of recurrence, specified for operative details and hernia type $(\mathrm{n}=137)$

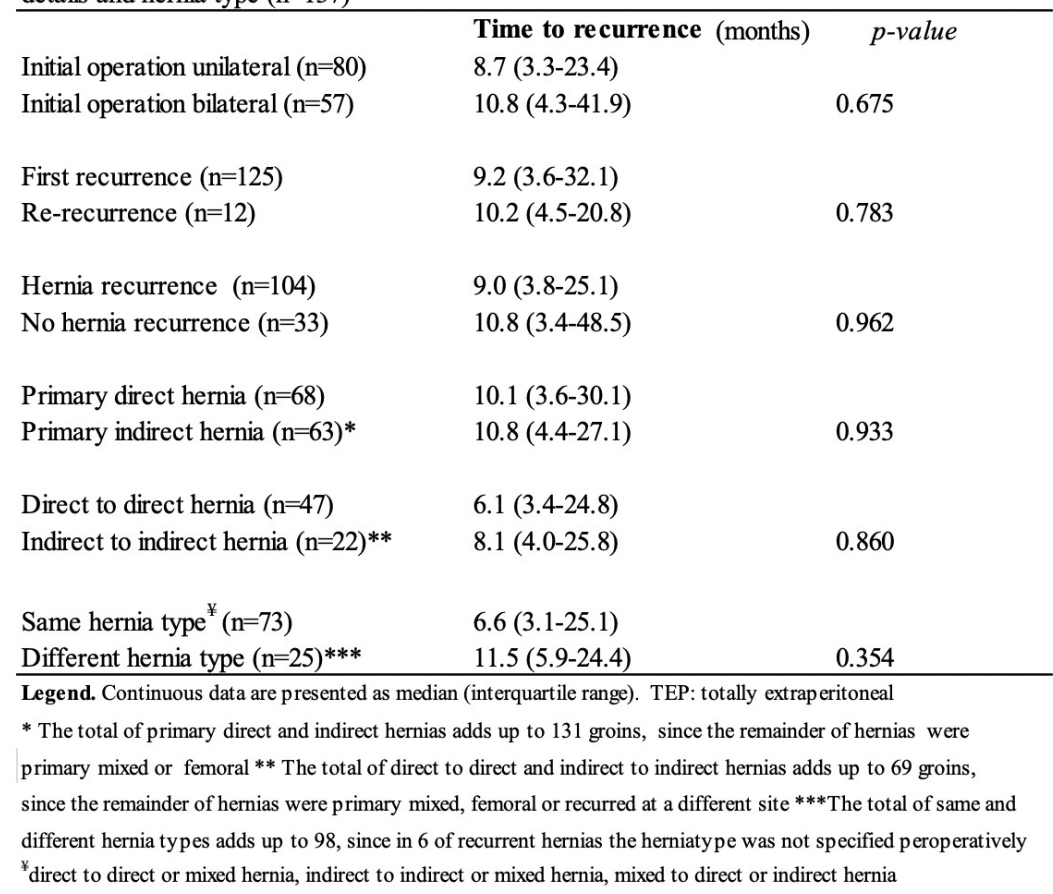

\section{DISCUSSION}

This retrospective analysis of 137 groins with recurrence-like symptoms after TEP inguinal hernia repair demonstrated true recurrent hernias were present in more than three quarter of cases. The majority of the recurrent hernias involved direct hernias after primary direct repair and developed in the first two years postoperatively. Patients presenting with large primary defects or undergoing bilateral TEP repair were at increased risk of developing a recurrence. Lipomas clinically mimicking hernia recurrences were present in nearly one fifth of reoperated groins.

Recurrence rates remain an important parameter in determining the clinical effectiveness of hernia surgery and represent a challenge for hernia surgeons. Even though currently reported recurrence rates after TEP inguinal hernia repair are low, auditing own outcomes by analyzing possible mechanisms of repair failure and recognition of causes of recurrence further 
facilitates prevention, elimination, and subsequent decrease of recurrences. Since this study was performed in a high-volume hernia clinic specialized in the TEP technique the influence of surgical inexperience or an uncompleted learning curve on development of recurrences after TEP repair allegedly was eliminated.

Recurrent hernias were present in $76 \%$ of all reoperated groins and the majority of recurrent hernias had developed at their initial hernia site (70\%), of which the greatest part involved direct hernias. A non-absorbable mesh of adequate (chemical and physical) properties, size and overlap that is adapted to the underlying tissue and enables good ingrowth without dislocation should prevent recurrence. ${ }^{4} \mathrm{~A}$ recurrence at the initial hernia site might be the consequence of failure in mesh positioning, mesh displacement or an inadequate mesh regarding the defect. Immediate or very early displacement might occur due to desufflation and removal of instruments, folding, lifting by hematoma or urinary retention. Late displacement may be caused by insufficient scar tissue ingrowth, mesh protrusion or mesh shrinkage due to contraction of fibrotic fibers. , $23^{23}$

Similar to the findinges of this study, Lamb et al. and Felix et al. found that respectively $67 \%$ and $76 \%$ of hernia recurrences were the same type as the original hernia. ${ }^{23,24}$ However, Lamb rapported on more reformation of indirect hernias. ${ }^{23} \mathrm{~A}$ study using data from the Danish Hernia Database conducted by Burcharth et al. also found a significant correlation between development of the same type of primary and recurrent hernia, most frequently involving direct hernias..$^{15}$ Lamb et al. and Burcharth et al. both described that development of the same type of hernia occurred significantly earlier postoperative, a finding that could not be confirmed from the results of this analysis. ${ }^{15,23}$

In more than four fifth of patients in whom a primary large defect was reported hernias recurred as their initial type. This percentage was considerably lower in cases where mesh fixation was performed, even though it does not become entirely clear from this analysis if all of the defects in the fixated cases were primary large. It is known that large defects increase the risk of development of the same type of hernia recurrence, with the currently used cut-off value for a large hernia defect of greater than 3 centimeter. ${ }^{9,25}$

Since the most frequently observed recurrences are direct to direct defects, with an increasing recurrence risk when the primary defect is large, one could consider (besides placement of tags) performing reduction of the dead space caused by the dilated transverse fascia in large direct hernia sacs as recommended in the recently updated guidelines for (endoscopic) hernia surgery. ${ }^{9-11}$ However, described benefits of this method are mainly prevention of hematoma or seroma, and no clear correlation with prevention of recurrence is described so far.10,11

More than $40 \%$ of patients that presented with a recurrence initially underwent a bilateral procedure. Since approximately $16 \%$ of patients that undergo primary TEP repair in our hernia clinic present with primary bilateral hernias, these numbers imply that patients undergoing primary bilateral TEP hernia repair are at an increased risk of developing a recurrence. ${ }^{22}$ Even though one could hypothesize that overlap of two meshes during bilateral TEP repair would decrease the chance on development of (direct) recurrence, this particular overlap of mesh may also prevent adequate fixation of mesh in the surrounding tissue. In case of bilateral 
repair, especially in larger patients, we would like to emphasize the importance of usage of an adequate mesh size and to consider the option of mesh fixation.

The greatest part of recurrences was diagnosed within two years after TEP hernia repair. Previous research that reported on time to development of hernia recurrence yielded similar results. ${ }^{15,23,24,26}$ The studies performed by Lamb et al. and Liem et al. also state that the majority of hernia recurrences developed within the first two postoperative years after TEP inguinal hernia repair, as well as the randomized controlled trial conducted in this hernia clinic between 2010 and 2012.19,20,23,26

Isolated lipomas were found in $18 \%$ of cases and occurred more frequently after correction of indirect hernias. Herniation of retroperitoneal adipose tissue into the inguinal canal is a frequent intraoperative finding during TEP repair and reduction of only the hernia sac without appropriate treatment of this lipoma can lead to recurrence-like symptoms. Unawareness of this condition of persisting lipoma can result in placing the mesh posterior of herniated retroperitoneal fatty tissue during TEP repair. ${ }^{27}$ This study demonstrated the incidence of isolated lipomas clinically mimicking recurrences was significantly higher after correction of indirect hernias. It is possible that these lipomas developed after the primary operation, but also likely that the isolated lipomas observed in this study represent 'forgotten' lipomas overlooked at the initial repair. Previous studies have reported upon (untreated) lipomas causing a pseudorecurrence as well.24,27,28 Even though technically these isolated lipomas are no true recurrences, this finding is still clinically relevant since reoperation will still be required in cases where these lipomas cause (recurrence-like) complaints.

When looking at the procedures performed at reoperation, based on the intraoperative findings a greater number of patients received a mesh than would have been necessary. With regard to the 25 patients where only an isolated lipoma was found during the second procedure, 18 Lichtenstein procedures with placement of mesh, one Fabricius repair and six inguinal explorations with removal of lipoma were performed. The eight patients where no recurrence or lipoma was found all received a mesh at the second operation; seven patients underwent a Lichtenstein procedure and one patient underwent another TEP procedure. For the patients that solely presented with lipomas, strictly, only removal of this lipoma would have been necessary and for patients without recurrence or lipoma no additional operative actions would have been required after inspection. Placement of a mesh in these cases unnecessarily increased the chance of (chronic) pain complaints.

The role of imaging to overcome unnecessarily mesh placement or operation, remains unclear. In this study in nearly $60 \%$ of reoperated groins preoperative imaging was conducted, however in more than a quarter of cases preoperative imaging findings and findings upon reoperation were discrepant. Due to the retrospective character of this study, the indication for and relevance of the performed imaging did not become entirely clear in all cases. In some cases the radiological hernia was already clinically apparent, and the imaging seemed unnecessary. When imaging showed an isolated lipoma instead of a recurrence, in this study population there was still decided to reoperate. Also, not in all cases the radiological diagnosis was in line with the clinical suspicion, and in some cases decisions to reoperate were made irrespective of (negative) imaging findings. In the current guidelines clinical examination plus ultrasound 
is recommended most suitable for evaluating patients suspected of having recurrent groin hernias, if diagnostic doubts persist CT or MRI should be considered. ${ }^{9}$ However, up to this point solely two studies of low quality have addressed this role of imaging for groin hernia recurrence. ${ }^{29,30}$ In our opinion, further research needs to be done regarding this issue.

Strengths of this study are the performance of a long-term eleven-year analysis of all reoperated groins in a high-volume hernia clinic where extensive experience is present.

A limitation is the retrospective character of this analysis, as not for all patients all required information could be retrieved. In the operation reports of the initial TEP repairs no distinction was made in the type of lipoma (funicular or preperitoneal). Also, not for all groins the hernia type was clearly specified upon reoperation. The size of the hernia defect was not standardly measured or reported, therefore primary large defects may have been missed or defects that actaully measured less than three centimeters in diameter may have been wrongly classified as large in some cases. Due to the anterior approach that was used in the majority of reoperated cases possible reasons of recurrence (eg. mesh dislocation or shrinkage) were not possible to assess and therefore report. Another limitation of this study is that only the patients reoperated for their recurrence within our hernia clinic were analyzed. Literature points out true recurrence rates are difficult to obtain, and currently reoperation rates are used as a proxy for recurrence rates, under the assumption recurrences occur up to twice as common as reoperations. ${ }^{16}$ Within the time frame of this study, more recurrences may have developed that have not presented at the outpatient clinic, were not reoperated or were possibly reoperated in another center. The characteristics of these possible recurrences remain unknown.

The recommendations regarding primary TEP repair and the operative procedure for recurrent hernias that could be formulated based on these study results are mainly in line with the current guidelines for (endoscopic) inguinal hernia repair. ${ }^{7-11}$ In case of primary large direct and bilateral defects, mesh fixation should be considered. The entire hernia floor should be thoroughly inspected with mandatory performance of lipoma removal, in case of indirect hernias in particular, to minimalize overlooked hernias and/or lipomas at TEP repair. For recurrent hernias after previous posterior (TEP) inguinal hernia repair, an anterior approach is recommended, where it remains important to act on peroperative findings and to avoid unnecessary operative steps in case no true hernia recurrence is found. 


\section{REFERENCES}

1. Read RC.Herniology: past, present, and future. Hernia 2009;13:577-80.

2. Campanelli G, Canziani M, Frattini F et al. Inguinal hernia: State of the art. International Journal of Surgery 2008; 6 S1:S26-8.

3. Nathan JD, Pappas TN.An old condition with new solutions. Ann Surg 2003;238(S6): S148-57.

4. Kukleta JF.Causes of recurrence in laparoscopic inguinal hernia repair. J Minim Access Surg 2006;2(3):187-91.

5. Read RC.Crucial steps in the evolution of the preperitoneal approaches to the groin: an historical review. Hernia 2011;15(1):1-5.

6 McCormack K, Wake BL, Fraser C et al. Trans abdominal pre-peritoneal (TAPP) versus totally extraperitoneal (TEP) laparoscopic techniques for inguinal hernia repair: a systematic review. Hernia 2005;9(2):109-14.

7. Simons MP, Aufenacker T, Bay-Nielsen M et al.European Hernia Society guidelines on the treatment of inguinal hernia in adult patients. Hernia 2009;13(4): 343-403

8. Miserez M, Peeters E, Aufenacker T et al. Update with level 1 studies of the European Hernia Society guidelines on the treatment of inguinal hernia in adult patients. Hernia 2014;18(2):151-63.

9. HerniaSurge Group. International guidelines for groin hernia management. Hernia 2018 22(1):1-165.

10. Bittner R, Arregui ME, Bisgaard T et al. Guidelines for laparoscopic (TAPP) and endoscopic (TEP) treatment of inguinal hernia [International Endohernia Society (IEHS)]. Surg Endosc 2011 25(9):2773-2843.

11. Bittner R, Montgomery MA, Arregui E et al .Update of guidelines on laparoscopic (TAPP) and endoscopic (TEP) treatment of inguinal hernia (International Endohernia Society). Surg Endosc Other Interv Tech 2015 29(2):289-321.

12. Liem MSL, van der Graaf Y, van Steensel CJ et al. Comparison of conventional anterior surgery and laparoscopic surgery for inguinal hernia repair. N Engl J Med 1997;336:1541-7.

13. O'Reilly EA, Burke JP, O'Connell PR. A meta-analysis of surgical morbidity and recurrence after laparoscopic and open repair of primary unilateral inguinal hernia. Ann Surg 2012;255(5):846-53.

14. Langeveld HR, Van't Riet M, Weidema WF et al. Total extraperitoneal inguinal hernia repair compared with Lichtenstein (the LEVEL-Trial): a randomized controlled trial. Ann Surg 2010;251:819-24.

15. Burcharth J, Andresen K, Pommergaard HC et al. Recurrence patterns of direct and indirect inguinal hernias in a nationwide population in Denmark. Surgery 2014;155(1):173-77.

16, Sevonius D, Gunnarsson U, Nordin P et al. Recurrent groin hernia surgery. Br J Surg 2011;98(10):1489-94.

17. Scheuerlein $\mathrm{H}$, Schiller A, Schneider $\mathrm{C}$ et al. Totally extraperitoneal repair of recurrent inguinal hernia: results from 179 consecutive patients. Surg Endosc Other Interv Tech 2003;17(7): 1072-6. 
18. Kockerling F, Jacob D, Wiegank W et al. Endoscopic repair of primary versus recurrent male unilateral inguinal hernias:are there differences in the outcome? Surg Endosc 2016;30(3):1146-55.

19. Burgmans JP, Voorbrood CE, Simmermacher RK et al. Long-term results of a randomized double-blinded prospective trial of a lightweight (Ultrapro) versus a heavyweight mesh (Prolene) in laparoscopic total extraperitoneal inguinal hernia repair (TULP-trial). Ann Surg 2016;263(5):862-6.

20. Roos MM, Bakker WJ, Schouten N et al. Higher recurrence rate after endoscopic totally extraperitoneal (TEP) inguinal hernia repair with Ultrapro lightweight mesh. Ann Surg 2018;268(2):241-6.

21. Neumayer L, Giobbie-Hurder A, Jonasson $O$ et al. Open mesh versus laparoscopic mesh repair of inguinal hernia. N Engl J Med 2004;350(18):1819-27.

22. Schouten N, Simmermacher RK, van Dalen T et al. Is there an end of the "learning curve" of endoscopic totally extraperitoneal (TEP) hernia repair? Surg Endosc 2013;27(3):789-94.

23. Lamb ADG, Robson AJ, Nixon SJ. Recurrence after totally extraperitoneal laparoscopic repair: Implications for operative technique and surgical training. Surgeon 2006;4(5) 299-307.

24. Felix E, Scott S, Crafton B et al. Causes of recurrence after laparoscopic hernioplasty - a multicenter study. Surg Endosc 1998;12:226-31.

25. Miserez M, Alexandre JH, Campanelli G et al. The European hernia society groin hernia classification: simple and easy to remember. Hernia 2007 11(2):113-6.

26. Liem MSL, Van Duyn EB, Van der Graaf Y et al. Recurrences after conventional anterior and laparoscopic inguinal hernia repair: a randomized comparison. Ann Surg 2003;237:136-41.

27. Lau H, Loong F, Yuen WK et al. Management of herniated retroperitoneal adipose tissue during endoscopic extraperitoneal inguinal hernioplasty. Surg Endosc 2007;21(9):1612-16.

28. Nasr AO, Tormey S, Walsh TN. Lipoma of the cord and round ligament: an overlooked diagnosis? Hernia 2005;9(3):245-7.

29. Shpitz B, Kuriansky J, Werener M et al. Early postoperative evaluation of groins after laparoscopic total extraperitoneal repair of inguinal hernias. J Laparoendosc Adv Surg Tech A 2004;14(6):353-7.

30. Young J, Gilbert Al, Graham MF. The use of ultrasound in the diagnosis of abdominal wall hernias. Hernia 2007;11(4):347-51. 


\title{
CHAPTER 6
}

\section{Recurrence after totally extraperitoneal (TEP) inguinal hernia repair; the role of physical examination and ultrasound}

\author{
CV van Hessen \\ MM Roos \\ FBM Sanders \\ EJMM Verleisdonk \\ GJ Clevers \\ PHP Davids \\ JPJ Burgmans
}

Hernia 2019 September 3 


\section{ABSTRACT}

\section{Background}

Physical examination (PE) combined with ultrasound (US) is recommended to confirm a recurrent hernia. However, the evidence is rather weak. The aim of this study was to evaluate PE and appraise the added value of US in alleged recurrent inguinal hernias after totally extraperitoneal (TEP) inguinal hernia repair.

\section{Methods}

All adult patients that were re-operated for suspicion of a recurrent hernia after a primary unilateral or bilateral TEP between 2006 and 2017, were identified and investigated retrospectively. Patient characteristics, PE, additional imaging and intra-operative findings were registered. PE outcomes were compared with intra-operative findings in order to calculate the positive predictive value (PPV) of PE. In case of clinical doubt, the added value of US was evaluated by comparing US findings with the intra-operative findings.

\section{Results}

A total of 130 patients were re-operated for suspicion of 137 recurrent hernias. In 75 patients US was performed. PE was positive for an inguinal hernia in 101 groins (73.7\%), negative in 30 (21.9\%) and inconclusive in 6 (4.4\%). PE matched the operative findings in $75.2 \%$. The PPV of diagnosing a recurrent hernia (or lipoma) on PE was $97 \%$. In case of clinical doubt $(n=36)$, positive US matched the operative findings in 20 cases $(87.0 \%)$.

\section{Conclusion}

US does not necessarily need to be incorporated in the standard diagnostic work-up of a recurrent inguinal hernia. After PE alone, a recurrent hernia (or lipoma) can be diagnosed with a PPV of $97 \%$. Only in case of clinical doubt, US has additional value. 


\section{BACKGROUND}

Recurrence rates after inguinal hernia repair dropped dramatically since the introduction of mesh-repair. Endoscopic preperitoneal mesh placement techniques results in less chronic pain compared to open mesh-repair with comparable recurrence rates if performed by experienced surgeons. Albeit mesh use for endoscopic techniques in experienced hands, recurrences still occur and remain one of the most important long-term complications. ${ }^{2}$

In the majority of cases, the clinical features of recurrent inguinal hernias are similar to those of primary inguinal hernias. However, diagnosing a recurrent hernia can be difficult and uncertain in case of unremarkable clinical symptoms.

In the international guidelines for groin hernia management, physical examination (PE) combined with ultrasound (US) is suggested as most suitable to confirm the diagnosis of a recurrent groin hernia. In case of negative ultrasonography and diagnostic doubts, Magnetic Resonance Imaging (MRI) or Computed Tomography (CT) may be considered. This recommendation does not make a distinction between clinically highly suspicious recurrences and complaints in the groin after inguinal surgery with uncertain features. The evidence on diagnostic imaging for recurrent groin hernias is rather scarce and solely based on two low quality studies. ${ }^{3,4}$ Therefore, it is questionable to what extent US adds value to PE for diagnosing a recurrent hernia.

The aim of this study was to evaluate the role of PE and appraise the added value of US in alleged (and repaired) recurrent inguinal hernias after totally extraperitoneal (TEP) inguinal hernia repair.

\section{METHODS}

\section{Study design}

A retrospective analysis of all re-operated (alleged) recurrent groin hernias was performed on the data of a previous described study. ${ }^{5}$ The study was carried out in a high-volume hernia center in the Netherlands specialized in TEP inguinal repair (1200 TEP repairs performed annually). In this center the recurrence rates after two- and five-year follow-up are $0.8 \%$ and $1.1 \%$ respectively. ${ }^{6,7}$

\section{Patients}

All adult patients that were re-operated for suspicion of a recurrent hernia after a primary unilateral or bilateral TEP between 2006 and 2017, were identified and analyzed retrospectively. Details about the inclusion were published previously. ${ }^{5}$ Medical history taking and PE was carried out by specialized hernia surgeons. Groin US was performed upon request of the 
general practitioner (GP) before referral or requested by the surgeon if deemed necessary. To reflect daily practice, we deliberately chose to retrospectively include patients regardless of who requested the groin US (general practitioner or surgeon), where it took place and who performed the groin US. Decisions to reoperate were based on a clinical or radiological suspicion of a recurrent hernia, a clinical or radiological suspicion of an isolated lipoma assumed to cause the recurrence-like complaints, or in case of a strong wish of the patient. All patients were re-operated with an anterior approach, with or without mesh depending on the intra-operative findings.

\section{Outcomes}

Patient characteristics (age, sex, body mass index (BMI) and American Society of Anesthesiologists (ASA) classification), time to recurrence after TEP repair, outcomes of history, PE, and US and intra-operative findings were registered. The time to recurrence was defined as the period of time between the recurrence date and the primary TEP procedure. The date the suspected recurrence was diagnosed at the outpatient clinic was used as a substitute for the date of recurrence.

Outcomes of history and PE of all included patients were divided into "positive", "negative" or "inconclusive" for inguinal hernia, based on the information in the electronic medical record. In case of an observable bulge, palpable swelling or positive Valsalva manoeuvre, groins were scored "positive". In the event of inadequate or vague documentation, PE findings were still taken into account and scored solely based on the documented conclusion. For all included patients, PE was compared to the intra-operative findings and the positive predictive value (PPV) was calculated. The calculation of the PPV is based upon the assumption that all referred patients with an evident symptomatic recurrent hernia based on a positive PE, were operated. This assumption is based on a prior prospective double-blinded randomized controlled trial carried out at the Dutch Hernia Clinic, in which all the PE-based recurrences after TEP-repair with use of a heavyweight mesh were operated. ${ }^{6}$

Intra-operative findings were divided into "positive" or "negative" for inguinal hernia based on the operative reports. A protrusion through a weakness in the posterior wall of the inguinal canal (fascia transversalis) or the deep inguinal ring (hernia sac or fatty tissue) was classified as positive. Furthermore, in case of an isolated lipoma, solely fatty tissue (anterior cord lipoma) not originating from the deep inguinal ring, the intra-operative findings were scored as lipoma. However, since treatment was required (anterior approach, without mesh), lipomas were counted as positive.

US outcomes were divided into "positive", "negative" or "inconclusive" for inguinal hernia, based on the radiology report or referral letter. Consistent with the intra-operative findings, lipomas were counted as positive. For all patients with clinical doubt or negative PE, US outcomes were compared to the intra-operative findings. The added value of US was evaluated by comparing the positive US outcomes with the intra-operative findings. Given the low number of CTs and MRIs performed in our population, these imaging modalities were excluded from this analysis. 


\section{RESULTS}

Between January 2006 and December 2016, 130 patients were re-operated for suspicion of 137 recurrent hernias after TEP hernia repair. The median time to detection of recurrence was 9.4 months (IQR 3.6-25.5 months) (Tab/e 1). A total of 75 patients (57.7\%) underwent 79 groin US before being re-operated. Groin US was most commonly requested by the surgeon ( $n=61$, $77.2 \%)$.

Table 1. Patient characteristics $(\mathrm{n}=130)$

Sex, n (\%)
Male

Female

BMI $\left(\mathrm{kg} / \mathrm{m}^{2}\right)$

Age at alleged recurrence (years)

Location of initial hernia, $n(\%)$

Unilateral

Bilateral

Median time to recurrence* (months)
$80(62)$

125 (96)

5 (4)

$25.2(23.2-27.8)$

$57.2(45.7-66.8)$

$50(38)$

$9.4(3.6-25.5)$

Legend. Continuous data are presented as median (Interquartile range) BMI: Body Mass Index, * Displayed for total number of groins, since 7 patients developed a bilateral recurrence $(n=137)$

As regards clinical findings ( $n=137), 101$ groins (73.7\%) were scored positive based on PE, compared to 30 negative groins (21.9\%) (Tab/e 2). In 6 groins (4.4\%) PE was inconclusive. The clinical findings matched the operative findings in 75.2\% (98 groins with both positive PE and intra-operative findings and 5 groins with both negative PE and intra-operative findings). The PVV to detect a recurrent inguinal hernia (or lipoma) by PE was 97.0\%. PE was well described in 125 groins (91.2\%). In 94 groins there was as a visible or palpable bulge, in 25 groins there was no swelling or positive Valsalva manoeuvre and in 6 cases the surgeon had reason to doubt the vague swelling. In only 12 cases (8.8\%) PE was not thoroughly described and therefore scored based on the conclusion in the electronic medical record, of which 7 groins were scored positive and 5 groins were scored negative.

In case of clinical doubt or negative PE ( $n=36), 32$ groin US were requested. In 2 cases only CT or MRI was requested due to persistent doubt after a negative PE, without first requesting US. In 2 cases no US was requested at all. Unfortunately, data regarding the decision to skip US before requesting MRI or CT and data regarding the decision-making of the surgeon in the other two cases is lacking. 23 groin US (63.9\%) scored positive for inguinal hernia compared to 6 negative groins (16.7\%) (Table 3). In 3 groins (8.3\%) US was inconclusive (2 US were not 
Table 2. Interrelation between physical examination and perioperative findings $(n=137)$

\begin{tabular}{|c|c|c|}
\hline & $\begin{array}{c}\text { Positive perioperative findings }(\mathrm{n}=129) \\
\text { (Inguinal hernia / Lipoma) }\end{array}$ & Negative Perioperative findings (n=8) \\
\hline $\begin{array}{c}\text { Physical Examination + } \\
(\mathrm{n}=101,73.7 \%)\end{array}$ & $98(82 / 16)$ & 3 \\
\hline $\begin{array}{c}\text { Physical Examination }- \\
(\mathrm{n}=30,21.9 \%)\end{array}$ & $25(16 / 9)$ & 5 \\
\hline $\begin{array}{c}\text { Physical Examination +/- } \\
(\mathrm{n}=6,4.4 \%)\end{array}$ & $6(6 /-)$ & - \\
\hline
\end{tabular}

Table 3. Interrelation between US and perioperative findings in case of clinical doubt $(n=32)$

\begin{tabular}{|c|c|c|}
\hline & $\begin{array}{c}\text { Positive perioperative findings (n=27) } \\
\text { (Inguinal hernia / Lipoma) }\end{array}$ & Negative Perioperative findings ( $\mathrm{n}=5)$ \\
\hline $\begin{array}{c}\text { Ultrasound }+ \\
(\mathrm{n}=23)\end{array}$ & $20(16 / 4)$ & 2 \\
\hline $\begin{array}{c}\text { Ultrasound }- \\
(\mathrm{n}=6)\end{array}$ & $4(2 / 2)$ & - \\
\hline $\begin{array}{c}\text { Inconclusive } \\
(\mathrm{n}=3)\end{array}$ & $3(2 / 1)$ & \\
\hline
\end{tabular}

able to distinguish between the different tissues and 1 US had inadequate documentation). US findings matched the operative findings in 22 cases $(68.8 \%, 20$ groins with both positive ultrasound and intra-operative findings and 2 groins with both negative ultrasound and intraoperative findings). In case of clinical doubt the positive groin US matched perioperative findings in 20 groins (87\%). Since the aim was to appraise the added value of US, we did not compare US outcomes to the intra-operative findings in patients with a positive PE.

In 6 groins there was decided to operate, regardless of the negative PE and US. Unfortunately, data regarding the decision-making of the surgeon is lacking in two of these cases. The decision to operate in 1 groin was based on the strong whish of the patient and one groin was explored due to the persistent doubt of the surgeon, based on the patients pattern of complaints. In two cases a MRI was requested due to persistent doubt. Both MRI's showed a lipoma. MRI findings matched the perioperative findings in both cases.

In 3 cases there was decided to operate with a negative PE and an inconclusive US. Data regarding the decision-making of the surgeon is lacking in 1 of these cases. In 1 case a CT was requested and in 1 case a MRI was requested due to persistent doubt. Both CT and MRI showed an inguinal hernia, which was confirmed during surgery. 


\section{DISCUSSION}

A recurrent inguinal hernia can be a challenging clinical diagnosis. This study demonstrates that almost $60 \%$ of patients re-operated for suspicion of a recurrent hernia after a primary TEP, underwent US in advance. Regarding the clinical features, a positive PE matched the intraoperative findings in 97.0\% (PPV). Therefore in case of a positive PE, medical imaging does not necessarily have to be incorporated in the diagnostic work-up of a recurrent inguinal hernia. However, in case of clinical doubt, US is an appropriate modality.

The results of this study show an excess of groin US requested for the diagnosis of a recurrent inguinal hernia, since only 32 of the 79 US were requested out of clinical doubt. In the interest of cost-effectiveness of healthcare, this surplus of diagnostic imaging needs to be taken into account. Given the high PPV of PE, our findings suggest that doctors should show considerable restraint when it comes to confirming a clinically obvious recurrent inguinal hernia with US.

To our knowledge this is the first study focusing on the role of PE and the added value of US in recurrent hernias. However, the recently published HerniaSurge guidelines recommend PE and groin US combined as most suitable for the evaluation of a recurrent hernia. This statement is based on studies by Sphitz et al. and Young et al. ${ }^{3,4}$ Although the recommendation is put into perspective by the degree of evidence (weak), the provided studies for substantiation of the statement have small sample sizes, short follow-up or no comparison with intra-operative findings. Furthermore, the added value of US above PE alone in suspected recurrent inguinal hernias was not assessed.

Data described by Shpitz et al. are not comparable to our study as they aimed to evaluate clinical and US findings in the groin only during the early postoperative period (2-3 weeks) following TEP inguinal repair. According to the literature US assessment is less reliable in the first weeks postoperative, as US is frequently hampered by hematoma, seroma, swelling of the subcutaneous fat and local tenderness. ${ }^{8}$ Moreover, no information was provided regarding the potential re-operation or intra-operative findings, hence it is not possible to make any assessment of the PPV in this study.

Young at al. retrospectively analyzed 200 patients and studied the value of ultrasound as an adjunctive tool in the diagnosis of abdominal wall hernias in both pre-operative and postoperative patients following herniorrhaphy. These data however, are not comparable to our study, since our study focused on recurrences after TEP. Moreover, only 4 groins showed a recurrent inguinal hernia on US, hence there were very little intra-operative findings to compare with the US and assessment of PPV for US was not possible.

Although this study showed no additional value of ultrasound in case of a clinically apparent recurrent hernia, actual implementation of the results in daily care may be challenging. Since GP's only incidentally see patients with an alleged possible recurrent inguinal hernia, they might be less capable of making a correct diagnosis based on PE alone. Moreover, since only $22.8 \%$ of the groin US in our study were requested by GP's, training of GP's in performing adequate physical examination will be of limited value in reducing the number of US requested. 
There is more to gain through convincing surgeons not to request groin US in cases of clinically manifest recurrent inguinal hernias. However, surgeons may feel burdened by the remaining groin pain or the occurred recurrence, and want to confirm their suspicion in order to avoid reoperations on the basis of false positive PE findings. In line with this precaution, groin US can also be requested out of fear of being sued. In recent years there has been a shift in the way that doctors practice medicine and in several Western countries 'defensive medicine' is on the rise..$^{9,10}$

It is important to bear in mind that the study data are only applicable for a situation in which a recurrent hernia after TEP is treated with an anterior approach (Lichtenstein). In these cases both recurrent inguinal hernias and isolated lipomas can be treated. Although a second preperitoneal approach is not recommended, some authors suggest another posterior approach (Trans Abdominal Preperitoneal repair (TAPP) or re-TEP) in case of a recurrent hernia after TEP.11,12 Irrespective of scar tissue due to the initial operation and consequently a higher complication risk associated with another posterior approach, in these cases anterior cord lipomas could be missed.

Little research has been conducted to understand and analyze the role of MRI or CT in detecting recurrent hernias. The guidelines suggest that MRI or CT should be considered if diagnostic doubts exist after clinical examination and US. However, as for US, the guidelines do not make a distinction between the role of imaging in the early postoperative period ( $<3$ weeks) and in the period later on. Where groin US in the early postoperative setting frequently is not possible or inconclusive, CT assessment appears to be a helpful imaging modality to distinguish between most of the postoperative complications following prosthetic mesh repair. ${ }^{13}$ Based on the little data in this study, we could not determine the role of MRI or $\mathrm{CT}$ in the work-up of early or late recurrent inguinal hernia.

Strengths of this study are that this is the first study focusing solely on the role of PE and the added value of US in recurrent hernias and the fact that the results are based on a long-term 11-year analysis of all re-operated groins in a hernia clinic where considerable experience is present.

It is possible that a number of limitations have influenced the results obtained. Due to its retrospective character, it is plausible no uniformity was assessed regarding PE records. Not every PE was extensively reported, so caution must be applied. In cases where the GP requested groin US before referral to the outpatient clinic, the PE may have been biased by the surgeon being aware of the US outcomes.

A second limitation, due to the retrospective character of this study is the plausibility of observer bias: US was performed by different radiologists and we know that the result of US is highly dependent on the expertise of the radiologist. As stated in our methods, we deliberately chose to retrospectively include patients regardless of who requested the groin US (general practitioner or surgeon), where it took place and who performed the groin US, to reflect daily practice. 
Moreover, it cannot be ruled out that there were patients with positive PE without an operation. If a patient was not fit for surgery, if an inguinal hernia was asymptomatic or due to a strong wish of the patient, decisions could have been made to refrain from surgery. A future study with a prospective design including all patients examined for a potential recurrence, being examined by one experienced radiologist and being operated on, could confirm the data of this study to draw definitive conclusions.

It is questionable whether recurrent inguinal hernias, including (small) fatty lipomas, diagnosed on ultrasound always explain the (nonspecific) groin pain and if or when a second operation resolves complaints. The aim of this study was to evaluate the role of PE and US in recurrent like symptoms after TEP. However, it would be interesting to perform a prospective study on the current topic in the future, with long-term follow up, including the outcome of surgical intervention.

The recommendation in the world guidelines to diagnose an recurrent inguinal hernia with PE combined with US is debatable. Given the results we would strongly advocate to confide in PE by a specialized hernia surgeon.US does not necessarily need to be incorporated in the diagnostic work-up of a recurrent inguinal hernia. However, in case of clinical doubt, US is the appropriate modality.

\section{REFERENCES}

1. HerniaSurge Group (2018), International guidelines for groin hernia management, Hernia, 22(1):1165. doi: 10.1007/s10029-017-1668-x.

2. Kingsnorth A, LeBlanc K. Hernias. (2003) Hernias: Inguinal and incisional. Lancet. 362:1561-1571. doi: 10.1016/S0140-6736(03)14746-0

3. Shpitz B, Kuriansky J, Werener M, Osadchi A, Tiomkin V, Bugayev N, Klein E (2004). Early postoperative evaluation of groins after laparoscopic total extraperitoneal repair of inguinal hernias. J Laparoendosc Adv Surg Tech A. 14(6):353-357. doi:10.1089/lap.2004.14.353

4. Young J, Gilbert Al, Graham MF. (2007) The use of ultrasound in the diagnosis of abdominal wall hernias. Hernia. 11(4):347-351. doi:10.1007/s10029-007-0227-2.

5. Roos MM, van Hessen CV, Verleisdonk EJMM, Clevers GJ, Davids PHP, Voorbrood CEH, Simmermacher RKJ, Burgmans JPJ. (2018) An 11-year analysis of reoperated groins after endoscopic totally extraperitoneal (TEP) inguinal hernia repair in a high volume hernia center. Hernia. doi: 10.1007/s10029-018-1827-8.

6. Burgmans JP, Voorbrood CE, Simmermacher RK, Schouten N, Smakman N, Clevers G, Davids PH, Verleisdonk EM, Hamaker ME, Lange JF, van Dalen T (2016) Long-term results of a randomized double-blinded prospective trial of a lightweight (Ultrapro) versus a heavyweight mesh (Prolene) in laparoscopic total extraperitoneal inguinal hernia repair (TULP-trial). Ann Surg;263(5):862-6. doi: 10.1097/SLA.0000000000001579. 
7. Roos MM, Bakker WJ, Schouten N, Voorbrood CEH, Clevers GJ, Verleisdonk EJMM, Davids PHP, Burgmans JPJ (2018) Higher Recurrence Rate After Endoscopic Totally Extraperitoneal (TEP) Inguinal Hernia Repair With Ultrapro Lightweight Mesh: 5-Year Results of a Randomized Controlled Trial (TULP-trial) Ann Surg. 268(2):241-246. doi: 10.1097/SLA.0000000000002649.

8. Tonolini M, Ippolito S (2016) Multidetector CT of expected findings and early postoperative complications after current techniques for ventral hernia repair. Insights Imaging 7:541-551. doi: 10.1007/s13244-016-0501-x.

9. Vandersteegen T, Marneffe W, Cleemput I, Vandijck D, Vereeck L. (2016) The determinants of defensive medicine practices in Belgium. Health Econ Policy Law 12:363-86. doi: 10.1017/ S174413311600030X.

10. Assing Hvidt E, Lykkegaard J, Pedersen LB, Pedersen KM, Munck A, Andersen MK. (2017) How is defensive medicine understood and experienced in a primary care setting? A qualitative focus group study among Danish general practitioners. BMJ Open. 7(12): e019851. doi: 10.1136/ bmjopen-2017-019851.

11. Ertem M, Ozben V, Gok H, Ozveri E (2013) Relaparoscopic treatment of recurrences after previous laparoscopic inguinal hernia repair. Minim Invasive Surg 260131. doi: 10.1155/2013/260131.

12. Sharma A, Sarwal A. (2017) Surgical repair in recurrent inguinal hernia. Ann Laparosc Endosc Surg 2:97. doi: $10.21037 /$ ales.2017.05.03

13. Lacour M, Ridereau Zins C, Casa C, Venara A, Cartier V, Yahya S, Barbieux J, Aubé C. (2017) CT findings of complications after abcominal wall repair with prosthetic mesh. Diagnostic and Interventional Imaging 98, 517-528. doi: 10.1016/j.diii.2017.01.002. 


\title{
C H A P T E R 7
}

\section{Is young age a risk factor for chronic postoperative inguinal pain after endoscopic totally extraperitoneal (TEP) repair?}

\author{
WJ Bakker \\ CV van Hessen \\ EJMM Verleisdonk \\ GJ Clevers \\ PHP Davids \\ N Schouten \\ JPJ Burgmans
}

Hernia 2019 January 17 


\section{ABSTRACT}

\section{Background}

A generally known risk factor for developing chronic postoperative inguinal pain after inguinal hernia repair is young age. However, studies discussing young age as a risk factor are mainly based on open repairs. The aim of this study was to determine if young adults (age 18-30) are also more prone to experience chronic postoperative inguinal pain after totally extraperitoneal (TEP) inguinal hernia repair, compared to older adults (age $\geq 31$ ).

\section{Methods}

A prospective study was conducted in a high-volume TEP hernia clinic in 919 patients. Patients were assessed using the Numeric (Pain) Rating Scale, Inguinal Pain Questionnaire and Carolina Comfort Scale preoperatively, at 3 months, 1 year and 2 years after TEP mesh repair. The primary outcome was clinically relevant pain in young adults compared to older adults at 3 months follow-up. Secondary outcomes were pain 1 and 2 years postoperatively, the impact of pain on daily living, foreign body feeling and testicular pain. Furthermore, age-categories were analyzed to determine potential age dependent risk factors.

\section{Results}

Follow-up was completed in 867 patients. No significant difference was found between young adults and older adults for clinically relevant pain at 3 months follow-up $(p=0.723)$. At all follow-up time points no significant differences were found for clinically relevant pain, any pain, mean pain scores, the Inguinal Pain Questionnaire and the Carolina Comfort Scale. The subgroup analyses showed no age dependent risk factor.

\section{Conclusions}

Young age is not associated with a higher risk of chronic postoperative inguinal pain after endoscopic TEP hernia repair. 


\section{BACKGROUND}

Since the introduction of inguinal hernia repair with placement of a mesh, recurrences have decreased impressively.,2 Ever since, chronic postoperative inguinal pain (CPIP) has become the most common disabling complication of inguinal hernia repair. ${ }^{3} \mathrm{CPIP}$ is defined as inguinal pain lasting more than 3 months after surgery. ${ }^{4,5}$ Various studies report a wide spread of CPIP incidence after inguinal hernia repair ranging from $0.7-75 \% .^{5}$ The reported incidence of CPIP in patients after endoscopic totally extraperitoneal (TEP) repair is $12.4 \%{ }^{6}$ Daily activities of patients with CPIP are affected in $2-20 \%$ of patients. ${ }^{7}$ As summarized in the international guidelines, known risk factors for CPIP are young age, female gender, high preoperative pain level, early high postoperative pain, recurrent hernia and open repair. ${ }^{5}$ However, there is no consensus with regard to a definition of 'young age'. Most studies discussing young age as a risk factor for CPIP are based on open repair and relevant studies for endoscopic repair are barely available. ${ }^{7-11}$ Since endoscopic TEP hernia repair by experienced surgeons results in a significantly lower incidence of CPIP compared to open repair, it remains unclear if young age is a risk factor for CPIP after endoscopic TEP repair. ${ }^{5}$ The aim of this study was to determine if young adults (18-30 years) are more prone to experience CPIP after TEP repair compared to adults of $\geq 31$ years of age.

\section{METHODS}

\section{Study design}

This prospective study was carried out in a high-volume hospital with extensive experience in the endoscopic TEP hernia repair technique (Hernia Clinic Diakonessenhuis Utrecht/Zeist). Hernia repairs were analyzed from the database of a double-blind randomized controlled trial (TULP-trial) comparing a lightweight and heavyweight mesh in patients that underwent TEP inguinal hernia repair. Detailed methodology has been published previously. ${ }^{14-17}$ Pre- and postoperative data regarding the presence of chronic pain up to 2 years after TEP repair at 4 time points were prospectively registered. For current analyses the patients in whom 3 months follow-up (minimum for CPIP) was completed were selected. The patients were included between March 2010 and October 2012. Informed consent was obtained in all patients. The study was approved by the regional Medical Ethics Committee (VCMO, Nieuwegein, the Netherlands) and the local ethics board of the hospital.

\section{Patients}

Patients deemed eligible for inclusion were male, over 18 years of age, with a primary, reducible, unilateral inguinal hernia and no contraindications for endoscopic TEP repair. Exclusion criteria were patients with collagen or connective tissue disorders and patients who were unlikely to complete the follow-up regimen since their understanding of the language was insufficient or they had no fixed address. 


\section{Intervention}

All patients underwent endoscopic TEP repair with tension-free placement of either a lightweight mesh (Ultrapro;, Ethicon, Johnson \& Johnson company, Amersfoort, The Netherlands) or a heavyweight mesh (Prolene", Ethicon, Johnson \& Johnson Company, Amersfoort, The Netherlands) (for specifications meshes, see: TULP-trial) [14-17]. Fixation of the mesh was not performed. All procedures were performed under general anesthesia. All patients were operated by 4 surgeons with extensive experience ( $>500$ procedures/surgeon) in performing the TEP inguinal hernia repair.

\section{Postoperative management, outcomes and follow-up}

Patients were routinely discharged on the day of surgery, unless complications prohibited early discharge. Patients were advised to take analgesics when in pain, and strenuous physical activity was discouraged during the first week postoperatively. No restrictions were given for activities of daily living.

The primary outcome of this study was clinically relevant pain (NRS 3-10) during rest in 18-30 year old patients compared to patients $\geq 31$ years old at 3 months after endoscopic TEP hernia repair. Secondary outcomes were CPIP (clinically relevant and any pain) during rest 1 and 2 years after TEP, the impact of pain on daily living, foreign body feeling and testicular pain in young adults compared to older adults. Furthermore, a subgroup analysis of age categories (mostly 10 years per category) was performed to determine a potential age dependent risk factor for the development of CPIP.

Patients visited the outpatient clinic at 3 months and 1 year for physical examination by a specialized hernia surgeon. Patients were approached by telephone if pain, discomfort or a bulge in the groin was reported in the 2 year questionnaires and offered a clinical appointment if required. Information regarding the, at the time, current presence of pain and the impact on daily living was obtained through questionnaires preoperatively and at 3 months, 1 and 2 years after TEP repair. Pain was measured using the Numeric (Pain) Rating Scale (NRS, a scale of $\mathrm{O}=$ no pain, 10 = worst imaginable pain, Dutch). NRS scores were reported during rest. Based on a recent systematic review pain intensity was categorized as mild (NRS 1-2), moderate (NRS 3-6) or severe (NRS 7-10) [18]. Moderate and severe pain (NRS 3-10) were considered clinically relevant. The Dutch versions of the Carolina Comfort Scale (CCS) and Inguinal Pain Questionnaire (IPQ) were used to assess the impact of pain on daily life activities $[19,20]$. These are both recommended hernia-specific measurement tools incorporating assessments of both pain intensity and quality of life (QOL). ${ }^{18}$ The CCS is a validated herniaspecific QOL questionnaire with 23 five-point scale questions of 0 being 'no pain, foreign body feeling or mechanical impairment' and 5 being 'terrible pain, foreign body feeling or mechanical impairment' (maximum total of points $=115$ ) $\cdot^{19,21}$ The IPQ uses a 7 step fixed-point rating scale to assess the impact of pain. The questionnaire uses separate questions to report the current inguinal pain, the worst pain experienced during the preceding week and the interference of pain with daily activities. Since the IPQ and the CCS overlap on certain subjects 
and for the reason of the unpractical extent of the IPQ questionnaire, the questions regarding a description of the worst pain in the past week, the experience of foreign body feeling (mesh) and pain in the testicle on the operated site were considered most relevant for this study. For the description of the worst pain in the past week, step 4-7, in which pain cannot be ignored and interferes with daily activities or worse, were considered clinically relevant. ${ }^{20}$

\section{Statistical analysis}

Sample size calculation and power analysis were performed using R, version 3.5.1 (The $R$ Foundation for Statistical Computing, Vienna, Austria). The hypothesis was that the incidence of clinically relevant chronic pain (proportional outcome) is higher in young adults (18-30 years) than in older adults (>30 years), as for open repairs and as described in the international guidelines. The analysis was based on the study by Langeveld et al., which is the only other study that analyzes the prognostic value of age for CPIP in TEP (and Lichtenstein) repair patients, and uses a proportional outcome. ${ }^{22}$ From the proportions encountered in their study ( $43.3 \%$ in the $18-40$ group versus $23.9 \%$ in the $>40$ group), with a two-sided alpha of 0.05 and a power of 0.80 , a total of 92 patients were to be included in each allocation group. However, regarding the study design of a secondary analysis of a previous conducted trial, the number of patients analyzed is fixed to 64 patients in the smallest group (age 18-30) and 855 patients in the other group (age >30). From the study of Langeveld et al. a power of 0.65 was determined for a group $n=64.22$

Outcome analyses were performed using SPSS statistical software, version 24 (IBM Corp, Armonk, NY). Descriptive statistics were used for baseline data. The incidence of clinically relevant pain and any pain at the 5 different time points were compared by means of chi-square analyses or a Fisher exact test. A univariate analysis was performed for possible confounders (mesh type, BMI, severe preoperative pain, hernia type and operation time). Variables with a $\mathrm{p}$-value of $<0.25$ in any of the relevant follow-up time points were subsequently entered in a multivariable analysis by means of a binary logistic regression in addition to age of 18-30 years to correct for confounding factors for the association between age of 18-30 years and CPIPexperience. The effects of the subgroups of age on clinically relevant pain were described using the relative risk $(\mathrm{RR})$ ratio with the $95 \%$ confidence interval $(\mathrm{Cl})$. For other endpoints, the Student $t$ test (normally distributed continuous), Mann-Whitney $U$ analysis (not normally distributed continuous) or chi-square analysis (categorical variables) was used. A p-value of $<0.05$ (two-sided) was considered significant.

\section{RESULTS}

Three month follow-up in the TULP-trial was completed in 919 patients, with a median age of 55 (IQR 44-64) (Table 1). Follow-up information at 1 and 2 years after TEP repair was available in 894 and 867 patients respectively (Figure 1). Significantly more indirect inguinal hernias were seen in young adults compared to older adults $(p=0.008)$. There was no significant difference in mesh distribution (Ultrapro" versus Prolene") for all age categories. 


\begin{tabular}{lc|ccc}
\hline \hline & $\begin{array}{c}\text { All patients } \\
(n=919)\end{array}$ & $\begin{array}{c}\text { Age } 18-30 \\
(n=64)\end{array}$ & $\begin{array}{c}\text { Age } \geq 31 \\
(n=855)\end{array}$ & $p$-value \\
\hline Age, years, median (IQR) & $55(44-64)$ & $25(23-28)$ & $57(46-64)$ & \\
BMI, kg/m ${ }^{2}$, mean (SD) & $24.9(2.6)$ & $22.9(2.3)$ & $25.0(2.6)$ & $0.000^{*}$ \\
Side, n (\%) & & & 0.683 \\
$\quad$ Left & $394(42.9)$ & $29(45.3)$ & $365(42.7)$ & \\
$\quad$ Right & $525(57.1)$ & $35(54.7)$ & $490(57.3)$ & \\
Hernia type, n (\%) & & & $0.008^{*}$ \\
$\quad$ Medial & $234(25.5)$ & $6(9.5)$ & $228(26.7)$ & \\
$\quad$ Lateral & $678(73.9)$ & $57(90.5)$ & $621(72.7)$ & \\
$\quad$ Femoral & $5(0.5)$ & $0(0)$ & $5(0.6)$ & \\
Mesh, n (\%) & & & 0.734 \\
$\quad$ Ultrapro & & & \\
$\quad$ Prolene & & & \\
Operation time, minutes, median (IQR) & $19(15-23)$ & $20(17-23)$ & $19(15-23)$ & 0.496 \\
\hline
\end{tabular}

IQR, Inter Quartile Range; BMI, Body Mass Index; SD, Standard Deviation; * $p<$ 0.05

Figure 1. Flowchart

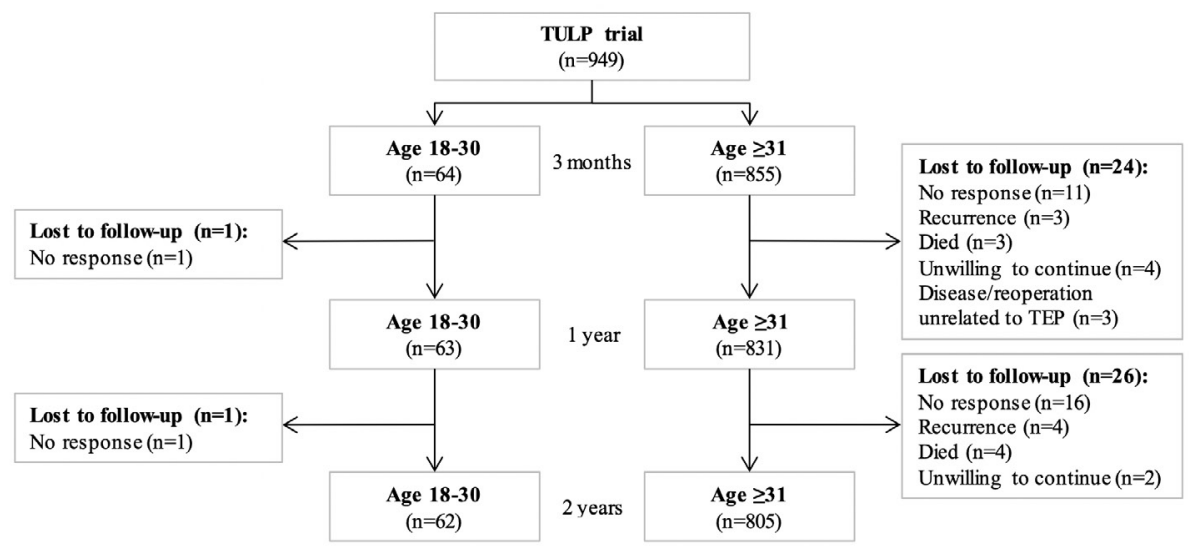

Clinically relevant pain (NRS 3-10) was present preoperatively and at 3 months, 1 and 2 years in, respectively, 345, 33, 24 and 31 patients. Preoperatively, clinically relevant pain was present in $43.8 \%$ of young adults versus $37.1 \%$ of patients $\geq 31$ years of age. Three months postoperatively, $1.6 \%$ of patients $18-30$ years of age complained of clinically relevant pain compared to $3.7 \%$ of patients $\geq 31$ years $s(p=0.723)$. For the preoperative data and all follow-up time points no significant difference was found for our secondary outcomes; clinically relevant pain at 1 and 2 years of follow-up, any pain (NRS 1-10), mean pain scores and the CCS. (Table 2) 
Table 2. Pain results

\begin{tabular}{|c|c|c|c|}
\hline NRS, mean (SD) & $18-30$ & $\geq 31$ & $p$-value \\
\hline Pre-operative & $2.53(2.3)$ & $2.34(2.4)$ & 0.408 \\
\hline 3 months & $0.17(0.5)$ & $0.37(1.0)$ & 0.145 \\
\hline 1 year & $0.19(0.6)$ & $0.24(0.9)$ & 0.963 \\
\hline 2 years & $0.21(0.7)$ & $0.25(0.8)$ & 0.695 \\
\hline \multicolumn{4}{|c|}{ Clinically relevant pain (NRS 3-10), n (\%) } \\
\hline Pre-operative & $28(43.8)$ & $317(37.1)$ & 0.288 \\
\hline 3 months & $1(1.6)$ & $32(3.7)$ & 0.723 \\
\hline 1 year & $1(1.6)$ & $23(2.8)$ & 1.000 \\
\hline 2 years & $2(3.2)$ & $29(3.6)$ & 1.000 \\
\hline \multicolumn{4}{|c|}{ Any pain (NRS $1-10), \mathrm{n}(\%)$} \\
\hline Pre-operative & $49(76.6)$ & $604(70.6)$ & 0.314 \\
\hline 3 months & $8(12.5)$ & $168(19.6)$ & 0.161 \\
\hline 1 year & $7(11.1)$ & $93(11.2)$ & 0.982 \\
\hline 2 years & $7(11.3)$ & $105(13.0)$ & 0.692 \\
\hline \multicolumn{4}{|c|}{ Carolina Comfort Scale*, mean points (SD) } \\
\hline Pre-operative & $15.5(14.5)$ & $14.4(14.9)$ & 0.396 \\
\hline 3 months & $4.1(12.0)$ & $3.0(8.0)$ & 0.761 \\
\hline 1 year & $0.9(3.7)$ & $1.8(6.1)$ & 0.094 \\
\hline 2 years & $2.0(7.1)$ & $1.7(5.6)$ & 0.652 \\
\hline
\end{tabular}

NRS, Numeric Rating Scale; SD, Standard Deviation

* Pre-operative maximum 75 points, other time points maximum 115 points.

Correcting for confounding factors did not change this finding for both clinically relevant pain and any pain at any of the 3 follow-up time points (Tab/e 3). A higher BMI, severe preoperative pain and the use of an Ultrapro lightweight mesh remained as independent predictors for clinically relevant pain at 3 months.

Table 3. Multivariable analysis

\begin{tabular}{|c|c|c|c|c|c|c|c|c|c|}
\hline \multirow[b]{2}{*}{ Any pain } & \multicolumn{3}{|c|}{3 months } & \multicolumn{3}{|c|}{1 year } & \multicolumn{3}{|c|}{2 years } \\
\hline & OR & $\mathrm{CI}(95 \%)$ & $p$-value & OR & $\mathrm{CI}(95 \%)$ & $p$-value & OR & CI $(95 \%)$ & $p$-value \\
\hline Age $18-30$ & 0.66 & $0.30-1.43$ & 0.293 & 1.29 & $0.56-2.99$ & 0.554 & 0.94 & $0.41-2.17$ & 0.890 \\
\hline Ultrapro mesh ${ }^{\circledR}$ & 0.97 & $0.70-1.35$ & 0.847 & 1.05 & $0.69-1.59$ & 0.835 & 1.38 & $0.92-2.07$ & 0.116 \\
\hline BMI & 1.06 & $0.99-1.13$ & 0.085 & 1.12 & $1.03-1.21$ & $0.007 *$ & 1.04 & $0.97-1.13$ & 0.286 \\
\hline Severe pre-operative pain & 1.80 & $0.81-4.03$ & 0.150 & 2.54 & $1.05-6.16$ & $0.038^{*}$ & 1.42 & $0.53-3.82$ & 0.491 \\
\hline Operation time & 0.99 & $0.97-1.02$ & 0.591 & 1.01 & $0.98-1.04$ & 0.703 & 1.01 & $0.98-1.04$ & 0.689 \\
\hline \multicolumn{10}{|l|}{ Clinically relevant pain } \\
\hline Age $18-30$ & 0.66 & $0.09-5.03$ & 0.685 & 0.99 & $0.12-7.88$ & 0.992 & 1.24 & $0.27-5.62$ & 0.782 \\
\hline Ultrapro mesh ${ }^{\circledR}$ & 1.28 & $0.63-2.60$ & 0.501 & 2.98 & $1.16-7.65$ & $0.023 *$ & 1.97 & $0.91-4.27$ & 0.087 \\
\hline BMI & 1.21 & $1.07-1.36$ & $0.003^{*}$ & 1.23 & $1.06-1.42$ & $0.007 *$ & 1.09 & $0.95-1.25$ & 0.227 \\
\hline Severe pre-operative pain & 2.77 & $0.78-9.88$ & 0.116 & 4.12 & $1.11-15.23$ & $0.034 *$ & 6.45 & $2.23-18.62$ & $0.001 *$ \\
\hline Operation time & 1.00 & $0.95-1.06$ & 0.990 & 0.99 & $0.92-1.05$ & 0.686 & 1.03 & $0.99-1.08$ & 0.158 \\
\hline
\end{tabular}


Concerning the IPQ question of describing the worst pain in the past week no significant differences were detected between young and older adults for clinically relevant pain (step 4-7) both preoperatively and at any time point postoperatively. Neither were significant differences detected for hindrance of foreign body feeling (mesh) or the experience of pain in the testicle on the operated site.

Analysis of the 7 subgroups of age showed that patients 41-51 years old had a significantly higher relative risk (RR) of more clinically relevant pain preoperatively and at 3 months postoperatively compared to the rest of the sample size. One year after TEP repair, this applied to patients $31-40$ years of age. (Tab/e 4)

Table 4. Relative risk ratio for experience of clinically relevant pain (NRS 3-10) for different age-categories

\begin{tabular}{|c|c|c|c|c|}
\hline \multirow[b]{2}{*}{ Age-category } & Pre-operative & 3 months & 1 year & 2 years \\
\hline & RR $(95 \%$ CI $)$ & RR $(95 \% \mathrm{CI})$ & RR $(95 \% \mathrm{CI})$ & RR $(95 \%$ CI $)$ \\
\hline $18-30 \quad(n=64)$ & $1.18(0.88-1.58)$ & $0.42(0.06-3.01)$ & $0.57(0.08-4.17)$ & $0.90(0.22-3.67)$ \\
\hline$(n=112)$ & $0.97(0.75-1.26)$ & $1.94(0.86-4.36)$ & $2.53(1.03-6.23)^{*}$ & $1.53(0.60-3.88)$ \\
\hline$(n=185)$ & $1.24(1.03-1.49)^{*}$ & $2.27(1.14-4.52)^{*}$ & $1.63(0.69-3.87)$ & $1.62(0.76-3.45)$ \\
\hline$(n=229)$ & $0.84(0.68-1.03)$ & $0.67(0.28-1.60)$ & $0.60(0.21-1.74)$ & $0.58(0.23-1.49)$ \\
\hline$(n=241)$ & $0.95(0.78-1.15)$ & $0.50(0.20-1.29)$ & $0.92(0.37-2.30)$ & $0.94(0.43-2.07)$ \\
\hline$(\mathrm{n}=78)$ & $0.92(0.67-1.26)$ & $0.70(0.17-2.85)$ & $\mathrm{N} / \mathrm{A}$ & $0.74(0.18-3.04)$ \\
\hline$(\mathrm{n}=10)$ & $1.34(0.72-2.50)$ & $\mathrm{N} / \mathrm{A}$ & $\mathrm{N} / \mathrm{A}$ & $\mathrm{N} / \mathrm{A}$ \\
\hline
\end{tabular}

* Confidence interval does not include 1.00 


\section{DISCUSSION}

This prospective study with 2 years of follow-up demonstrates that in a center of expertise, young adults (18-30 years) do not experience more CPIP after TEP hernia repair compared to older adults ( $\geq 31$ years). Moreover, no age dependent risk factor for the development of CPIP could be identified in our subgroup analysis.

Several studies have shared their findings on the influence of age on CPIP. Nevertheless, up to our knowledge this is the first study focusing on young age as a risk factor for CPIP after endoscopic inguinal hernia surgery. Our findings of CPIP (NRS 1-10) in 12.5\% at 3 months after surgery decreasing to $11.3 \%$ over time for age $18-30$, and $19.6 \%$ decreasing to $13.0 \%$ over time for age $\geq 31$, is conform with the reported $12.4 \%$ of TEP patients with CPIP of a recent systematic review and meta-analysis. ${ }^{6}$

One other study by Langeveld et al. (2015) had a comparable study design to ours, although patients underwent TEP and Lichtenstein, the sample size was smaller and pain was only assessed as a dichotomous value (yes/no). ${ }^{22}$ They concluded that younger patients (18-40 years) presented more often with CPIP than middle-aged or elderly patients and TEP did not reduce the pain incidence. They however neglected to correct for confounders, since in their study younger patients also had more frequent preoperative pain and the intensity of pain was higher during the first 3 postoperative days, which are known risk factors for the development of CPIP.

The guidelines defined their statement of young age being a risk factor for CPIP mainly based on studies of patients after open repair.,11,12 Only 2 studies investigated pain exclusively after endoscopic repair. 9,10 Dickinson et al. (2008) described a significant correlation of young age and CPIP after TEP, however young age was defined as $<50$ years. ${ }^{9}$ Lau et al. (2004) did not address chronic pain, but found more acute pain after TEP repair in patients $<65$ years of age in the first days after surgery..$^{10}$ Liem et al. (2003) described a randomized comparison of laparoscopic and open inguinal hernia repairs with 5 years of follow-up. ${ }^{8}$ Comparable to our study, age was not identified as a significant influence on the development of CPIP. Only preoperative pain, open repair and an intraoperative lesion of the ilioinguinal nerve were identified as predictors for CPIP.

The subgroup analyses of the current study identified a significantly higher RR for CPIP for the age category 41-50 years, preoperatively and at 3 months. This is most likely due to the before mentioned risk factor preoperative pain and not the age category, especially since significance fades at 1 and 2 years of follow-up. We have difficulty explaining the significantly higher RR seen in patients in age category 31-40 years 1 year after TEP repair, although probably the group sample size and multiple outliers resulted in the outcome.

This study did not show more CPIP, foreign body feeling or worse scores concerning QOL in young adults compared to older adults after TEP mesh repair. However, if mesh placement should always be advised in young patients remains questionable. Mesh placement reduces recurrences by reinforcement of the weak inguinal floor. However, in young adults a different etiology of a patent processus vaginalis might be causative for an indirect inguinal hernia 
without a weakened posterior wall or large defect.22,23 The more frequent appearance is confirmed by both the database of Langeveld et al. (2015) and our database that show significantly more indirect inguinal hernias in young adults. ${ }^{22}$

In children ( $<18$ years), with a patent processus vaginalis as the primary cause of an indirect inguinal hernia, an open herniotomy is the procedure of choice. In patients $>30$ years of age with an inguinal hernia sufficient evidence has been provided that an open herniotomy is accompanied by high recurrence rates which necessitates the use of a mesh. ${ }^{5}$ For patients of 18-30 years the available evidence is weak [5]. A recent retrospective study by van Kerckhoven et al. (2016) found recurrence rates after open herniotomy of $0 \%$ in patients aged $18-25,2.7 \%$ in patients aged $18-30$ years and $4.7 \%$ in patients aged $18-40$ years, and suggested open herniotomy as a possible treatment of choice in young adults. ${ }^{25}$ Osifo and Irowa (2008) found 1 recurrence $(0.3 \%)$ after open herniotomy in patients with indirect inguinal hernias (mean age $25 \pm 5.3$ years, range 12-45) with 1-5 years of follow-up. ${ }^{26}$ Well-designed prospective trials with adequate follow-up duration for the detection of recurrences are lacking and especially in direct inguinal hernias (current study $9.5 \%$ of patients) higher recurrence rates are to be expected. In our sample of patients no recurrences were detected in young adults (age 18-30) after TEP mesh repair with 5 years of follow-up..$^{16}$

Therefore, we recommend performing TEP inguinal hernia repair with placement of a mesh in patients of 18-30 years of age. Nevertheless, open herniotomy could be an alternative in young adults who are unwilling to undergo mesh repair. However, higher recurrence rates after open herniotomy are seen and to be expected compared to TEP mesh repair and hence patients should be informed. Randomized controlled trials to detect recurrence differences between TEP and open herniotomy require lengthy follow-up and inordinate sample sizes. Detecting differences in pain might be virtually impossible. Therefore, we see no necessity for randomizing open herniotomy versus endoscopic mesh repair in young adults.

A strength of this study is the long-term follow-up in a large sample of patients with the use of recommended and validated questionnaires aimed at analyzing (chronic) pain and interference with daily activities. ${ }^{18}$ Moreover, the current study provided a fairly pure analysis of the risk factor age. The study design filtered out other risk factors for CPIP development as female gender, recurrences and open repair, and correction for the remaining confounders through a multivariable analysis was performed. Furthermore, the study was conducted in a single, highly experienced TEP center. Comparable to TEP repair, outcomes of chronic pain in young adults after transabdominal preperitoneal (TAPP) repair are underreported. From multiple high-volume comparisons, the recently published international guidelines concluded that TAPP and TEP show similar complication rates for, amongst other outcomes, inguinal nerve lesions, chronic pain and recurrences. ${ }^{5}$ Presuming there is no age-related influence on the comparability of TEP and TAPP, extending the current outcomes to laparo-endoscopic inguinal hernia repairs seems justified.

A limitation to this study is that it was not primarily aimed at distinguishing between age categories. The original TULP-trial was randomized between lightweight and heavyweight mesh and powered for the detection of pain differences, however not for age categories resulting in different sample sizes. The sample size of 64 in the age-category $18-30$ resulted in 
a power of 0.65 , which is below the optimal standard. In a high-volume TEP center 2.5 years of including yielded 64 patients in this age-category. Therefore, achieving a power of $>0.80$ will be challenging. Presumably due to the difficulty of establishing sample sizes for optimal power in this age-category, this is the first study to analyze patients in the age-category 18-30 years after TEP repair. Maintaining prospective inguinal hernia registration databases is advised and might provide adequate sample sizes for analyses of higher power in time. Moreover, since it was not our primary outcome our subgroup analysis of age-categories was not corrected for confounders, including the type of mesh used. Yet, the mesh distribution showed no significant difference for each of the 7 subgroups separately in comparison with the rest of the sample population.

In conclusion, age did not prove to be a risk factor for the development of CPIP after TEP mesh repair, which justifies this technique in patients $18-30$ years of age. 


\section{REFERENCES}

1. Lichtenstein IL (1987) Herniorrhaphy. A personal experience with 6,321 cases. Am J Surg 153:553-559

2. Langeveld HR, van't Riet M, Weidema WF, Stassen LP, Steyerberg EW, Lange J, Bonjer HJ, Jeekel J (2010) Total extraperitoneal inguinal hernia repair compared with Lichtenstein (the LEVEL-Trial): a randomized controlled trial. Ann Surg 251:819-824

3. Kehlet $\mathrm{H}$ (2008) Chronic pain after groin hernia repair. Br J Surg 95:135-136

4. Classification of chronic pain. Descriptions of chronic pain syndromes and definitions of pain terms. Prepared by the International Association for the Study of Pain, Subcommittee on Taxonomy(1986). Pain Suppl 3:S1-226

5. HerniaSurge Group (2018) International guidelines for groin hernia management. Hernia 22:1-165

6. Koning GG, Wetterslev J, van Laarhoven CJ, Keus F (2013) The totally extraperitoneal method versus Lichtenstein's technique for inguinal hernia repair: a systematic review with meta-analyses and trial sequential analyses of randomized clinical trials. PLoS One 8:e52599

7. Bay-Nielsen M, Perkins FM, Kehlet H, Danish Hernia Database (2001) Pain and functional impairment 1 year after inguinal herniorrhaphy: a nationwide questionnaire study. Ann Surg 233:1-7

8. Liem MS, van Duyn EB, van der Graaf Y, van Vroonhoven TJ, Coala Trial Group (2003) Recurrences after conventional anterior and laparoscopic inguinal hernia repair: a randomized comparison. Ann Surg 237:136-141

9. Dickinson KJ, Thomas M, Fawole AS, Lyndon PJ, White CM (2008) Predicting chronic post-operative pain following laparoscopic inguinal hernia repair. Hernia 12:597-601

10. Lau H, Patil NG (2004) Acute pain after endoscopic totally extraperitoneal (TEP) inguinal hernioplasty: multivariate analysis of predictive factors. Surg Endosc 18:92-96

11. Bay-Nielsen M, Nilsson E, Nordin P, Kehlet H, Swedish Hernia Data Base the Danish Hernia Data Base (2004) Chronic pain after open mesh and sutured repair of indirect inguinal hernia in young males. Br J Surg 91:1372-1376

12. Franneby U, Sandblom G, Nordin P, Nyren O, Gunnarsson U (2006) Risk factors for long-term pain after hernia surgery. Ann Surg 244:212-219

13. Bittner R, Arregui ME, Bisgaard T, Dudai M, Ferzli GS, Fitzgibbons RJ, Fortelny RH, Klinge U, Kockerling F, Kuhry E, Kukleta J, Lomanto D, Misra MC, Montgomery A, Morales-Conde S, Reinpold W, Rosenberg J, Sauerland S, SchugPass C, Singh K, Timoney M, Weyhe D, Chowbey P (2011) Guidelines for laparoscopic (TAPP) and endoscopic (TEP) treatment of inguinal hernia [International Endohernia Society (IEHS). Surg Endosc 25:2773-2843

14. Burgmans JP, Voorbrood CE, Schouten N, Smakman N, Elias S, Clevers GJ, Davids PH, Verleisdonk EJ, Hamaker ME, Simmermacher RK, van Dalen T (2015) Three-month results of the effect of Ultrapro or Prolene mesh on postoperative pain and well-being following endoscopic totally extraperitoneal hernia repair (TULP trial). Surg Endosc 29:3171-3178 
15. Burgmans JP, Voorbrood CE, Simmermacher RK, Schouten N, Smakman N, Clevers G, Davids PH, Verleisdonk EM, Hamaker ME, Lange JF, van Dalen T (2016) Long-term Results of a Randomized Double-blinded Prospective Trial of a Lightweight (Ultrapro) Versus a Heavyweight Mesh (Prolene) in Laparoscopic Total Extraperitoneal Inguinal Hernia Repair (TULP-trial). Ann Surg 263:862-866

16. Roos M, Bakker WJ, Schouten N, Voorbrood C, Clevers GJ, Verleisdonk EJ, Davids P, Burgmans J (2018) Higher Recurrence Rate After Endoscopic Totally Extraperitoneal (TEP) Inguinal Hernia Repair With Ultrapro Lightweight Mesh: 5-Year Results of a Randomized Controlled Trial (TULP-trial). Ann Surg

17. Schouten N, van Dalen T, Smakman N, Elias SG, Clevers GJ, Verleisdonk EJ, Davids PH, Burgmans IP (2012) The effect of ultrapro or prolene mesh on postoperative pain and well-being following endoscopic Totally Extraperitoneal (TEP) hernia repair (TULP): study protocol for a randomized controlled trial. Trials 13:76-6215-13-76

18. Molegraaf M, Lange J, Wijsmuller A (2017) Uniformity of Chronic Pain Assessment after Inguinal Hernia Repair: A Critical Review of the Literature. Eur Surg Res 58:1-19

19. Heniford BT, Walters AL, Lincourt AE, Novitsky YW, Hope WW, Kercher KW (2008) Comparison of generic versus specific quality-of-life scales for mesh hernia repairs. J Am Coll Surg 206:638-644

20. Franneby U, Gunnarsson U, Andersson M, Heuman R, Nordin P, Nyren O, Sandblom G (2008) Validation of an Inguinal Pain Questionnaire for assessment of chronic pain after groin hernia repair. Br J Surg 95:488-493

21. Nielsen K, Poelman MM, den Bakker FM, van der Ploeg T, Bonjer HJ, Schreurs WH (2014) Comparison of the Dutch and English versions of the Carolinas Comfort Scale: a specific quality-of-life questionnaire for abdominal hernia repairs with mesh. Hernia 18:459-464

22. Langeveld HR, Klitsie P, Smedinga H, Eker H, Van't Riet M, Weidema W, Vergouwe Y, Bonjer HJ, Jeekel J, Lange JF (2015) Prognostic value of age for chronic postoperative inguinal pain. Hernia 19:549-555

23. van Veen RN, van Wessem KJ, Halm JA, Simons MP, Plaisier PW, Jeekel J, Lange JF (2007) Patent processus vaginalis in the adult as a risk factor for the occurrence of indirect inguinal hernia. Surg Endosc 21:202-205

24. Jiang ZP, Yang B, Wen LQ, Zhang YC, Lai DM, Li YR, Chen S (2015) The etiology of indirect inguinal hernia in adults: congenital or acquired?. Hernia 19:697-701

25. van Kerckhoven G, Toonen L, Draaisma WA, de Vries LS, Verheijen PM (2016) Herniotomy in young adults as an alternative to mesh repair: a retrospective cohort study. Hernia 20:675-679

26. Osifo OD, Irowa OO (2008) Indirect inguinal hernia in Nigerian older children and young adults: is herniorrhaphy necessary?. Hernia 12:635-639 


\title{
CH A P T E R 8
}

\section{Antibiotic prophylaxis for prevention of postoperative wound infection in adults undergoing open elective inguinal or femoral hernia repair}

\author{
CC Orelio \\ CV van Hessen \\ FJ Sanches-Manuel \\ TJ Aufenacker \\ RJPM Scholten
}




\title{
Antibiotic prophylaxis for prevention of postoperative wound infection in adults undergoing open elective inguinal or femoral hernia repair
}

\author{
Claudia C Orelio ${ }^{1}$, Coen van Hessen², Francisco Javier Sanchez-Manuel ${ }^{3}$, Theodorus J Aufenacker ${ }^{4}$, Rob JPM Scholten ${ }^{5}$
}

${ }_{1}$ Research Support, Diakonessenhuis Utrecht, Utrecht, Netherlands. ${ }^{2}$ Liesbreukcentrum Nederland, Diakonessenhuis Utrecht, Utrecht, Netherlands. 3General and Digestive Surgery, Complejo Asistencial Universitario de Burgos. Universitary Burgos's Hospital, Burgos, Spain. ${ }^{4}$ Department of Surgery, Rijnstate Hospital, Arnhem, Netherlands. ${ }^{5}$ Cochrane Netherlands, Julius Center for Health Sciences and Primary Care, University Medical Center Utrecht, Utrecht University, Utrecht, Netherlands

\section{A B S T R A C T}

\section{Background}

Inguinal or femoral hernia is a tissue protrusion in the groin region and has a cumulative incidence of $27 \%$ in adult men and of $3 \%$ in adult women. As most hernias become symptomatic over time, groin hernia repair is one of the most frequently performed surgical procedures worldwide. This type of surgery is considered 'clean' surgery with wound infection rates expected to be lower than $5 \%$. For clean surgical procedures, antibiotic prophylaxis is not generally recommended. However after the introduction of mesh-based hernia repair and the publication of studies that have high wound infection rates the debate as to whether antibiotic prophylaxis is required to prevent postoperative wound infections started again.

\section{Objectives}

To determine the effectiveness of antibiotic prophylaxis in reducing postoperative (superficial and deep) wound infections in elective open inguinal and femoral hernia repair.

\section{Search methods}

We searched several electronic databases: Cochrane Registry of Studies Online, MEDLINE Ovid, Embase Ovid, Scopus and Science Citation Index (search performed on 12 November 2019). We also searched two trial registers and the reference list of included studies.

\section{Selection criteria}

We included randomised controlled trials comparing any type of antibiotic prophylaxis versus placebo or no treatment for preventing postoperative wound infections in adults undergoing inguinal or femoral open hernia repair surgery (tissue repair and mesh repair).

\section{Data collection and analysis}

Two review authors independently selected studies, extracted data and assessed risk of bias. We separately analysed results for two different surgical methods (herniorrhaphy and hernioplasty). Several studies revealed infection rates that were higher than the expected $5 \%$ for clean surgery and we therefore divided studies into two subgroups: high infection risk environments ( $\geq 5 \%$ infection rate); and low infection risk environments (<5\% infection rate). We performed meta-analyses with random-effects models. We analysed three outcomes: superficial surgical site infections (SSSI); deep surgical site infections (DSSI); and all postoperative wound infections (SSSI + DSSI). 


\section{Main results}

In this review update we identified and included 10 new studies. In total, we included 27 studies with 8308 participants in this review.

It is uncertain whether antibiotic prophylaxis as compared to placebo (or no treatment) prevents all types of postoperative wound infections after herniorrhaphy surgery (risk ratio (RR) $0.86,95 \%$ confidence interval (CI) 0.56 to 1.33; 5 studies, 1865 participants; very low quality evidence). Subgroup analysis did not change these results. We could not perform meta-analyses for SSSI or DSSI as these outcomes were not reported separately.

Twenty-two studies related to hernioplasty surgery (total of 6443 participants) and we analysed three outcomes: SSSI; DSSI; SSSI + DSSI.

Within the low infection risk environment subgroup, antibiotic prophylaxis as compared to placebo probably makes little or no difference for the outcomes 'prevention of all wound infections' (RR $0.71,95 \% \mathrm{Cl} 0.44$ to 1.14; moderate-quality evidence) and 'prevention of SSSI' (RR $0.71,95 \% \mathrm{Cl} 0.44$ to 1.17 , moderate-quality evidence). Within the high infection risk environment subgroup it is uncertain whether antibiotic prophylaxis reduces all types of wound infections ( $\mathrm{RR} 0.58,95 \% \mathrm{Cl} 0.43$ to 0.77 , very low quality evidence) or SSSI (RR $0.56,95 \% \mathrm{Cl} 0.41$ to 0.77 , very low quality evidence). When combining participants from both subgroups, antibiotic prophylaxis as compared to placebo probably reduces the risk of all types of wound infections ( $\mathrm{RR} 0.61,95 \% \mathrm{Cl} 0.48$ to 0.78 ) and SSSI (RR $0.60,95 \% \mathrm{Cl} 0.46$ to 0.78 ; moderatequality evidence).

Antibiotic prophylaxis as compared to placebo probably makes little or no difference in reducing the risk of postoperative DSSI (RR 0.65, $95 \% \mathrm{Cl} 0.26$ to 1.65 ; moderate-quality evidence), both in a low infection risk environment ( $\mathrm{RR} 0.67,95 \% \mathrm{Cl} 0.11$ to 4.13 ; moderate-quality evidence) and in the high infection risk environment ( $\mathrm{RR} 0.64,95 \% \mathrm{Cl} 0.22$ to 1.89 ; low-quality evidence).

\section{Authors' conclusions}

Evidence of very low quality shows that it is uncertain whether antibiotic prophylaxis reduces the risk of postoperative wound infections after herniorrhaphy surgery. Evidence of moderate quality shows that antibiotic prophylaxis probably makes little or no difference in preventing wound infections (i.e. all wound infections, SSSI or DSSI) after hernioplasty surgery in a low infection risk environment. In a high-risk environment, evidence of very low quality shows it is uncertain whether antibiotic prophylaxis reduces all wound infections and SSSI after hernioplasty surgery. Evidence of low quality shows that antibiotic prophylaxis in a high-risk environment may have little or no difference in reducing the risk of DSSI.

\section{PLAIN LANGUAGE SUMMARY}

\section{Can antibiotics as compared to placebo prevent postoperative wound infections after open groin hernia repair surgery?}

\section{Background}

Groin hernia is a weakness in the abdominal wall in the groin area, through which soft tissue or organs can protrude. Groin hernias occur often and therefore groin hernia repair is one of the most frequently performed surgical operations worldwide. It is considered a 'clean' surgical technique with low postoperative wound infections rates and administration of antibiotics to patients undergoing open hernia repair surgery is therefore not generally recommended. Up to the 1990s, suture-based hernia repair (herniorrhaphy) was the method of choice. From then onwards hernia repair with a synthetic mesh (hernioplasty) gained increasing popularity and the debate as to whether antibiotics are needed to prevent postoperative wound infections started again.

\section{Investigation}

We searched the literature (12 November 2019) for randomised controlled trials comparing antibiotics versus placebo to prevent wound infections after open groin hernia repair surgery. We included both suture-based and mesh-type surgical methods. We divided infections into superficial and deep wound infections. Several studies revealed infection rates that were higher than the expected $5 \%$ for clean surgery. Therefore, we divided studies into a group with low infection rates (less than $5 \%$ ) and one with high infection rates (more than $5 \%$ ).

\section{Study characteristics and key results}

We identified five suture-based surgery studies and 22 mesh-type surgery studies. The suture-based studies were of very low methodological quality. The mesh-type surgery studies were of low to moderate methodological quality.

This review shows that antibiotics do not prevent the occurrence of any type of wound infections after suture-based hernia repair. For mesh-type hernia repair in a low infection risk environment, antibiotics probably make little to no difference in prevention of postoperative superficial wound infections. However, in a high infection risk environment it is uncertain whether antibiotics reduce the risk of superficial wound infection occurrence.

For deep wound infections, we show that antibiotics probably make little or no difference in reducing the risk in both a low and high infection risk environment. 


\section{Quality of the evidence}

Evidence of very low quality shows that it is uncertain whether antibiotics reduce the risk of postoperative wound infections after suturebased hernia repair. Evidence of moderate quality shows that that antibiotics probably make little or no difference in preventing superficial or deep wound infections after mesh-type hernia repair in a low infection risk environment. Evidence of (very) low quality shows that it is uncertain whether antibiotics reduce the risk of superficial wound infections, and antibiotics have little or no effect on deep wound infections after mesh-type hernia repair in a high infection risk environment. 
SUMMARY OF FINDINGS

Summary of findings 1. Antibiotic prophylaxis compared to placebo for prevention of postoperative wound infection in adults undergoing open inguinal or femoral herniorrhaphy surgery

Antibiotic prophylaxis compared to placebo for prevention of postoperative wound infection in adults undergoing open elective inguinal or femoral herniorrhaphy hernia repair

Patient or population: prevention of postoperative wound infection in adults undergoing open elective inguinal or femoral herniorrhaphy hernia repair Setting:

Intervention: antibiotic prophylaxis

Comparison: placebo

\begin{tabular}{|c|c|c|c|c|c|c|}
\hline \multirow[t]{2}{*}{ Outcomes } & \multicolumn{2}{|c|}{ Anticipated absolute effects ${ }^{\star}(95 \% \mathrm{Cl})$} & \multirow{2}{*}{$\begin{array}{l}\text { Relative effect } \\
(95 \% \mathrm{CI})\end{array}$} & \multirow{2}{*}{$\begin{array}{l}\text { № of partic- } \\
\text { ipants } \\
\text { (studies) }\end{array}$} & \multirow{2}{*}{$\begin{array}{l}\text { Certainty of the } \\
\text { evidence } \\
\text { (GRADE) }\end{array}$} & \multirow{2}{*}{$\begin{array}{l}\text { Com- } \\
\text { ments }\end{array}$} \\
\hline & Risk with placebo & Risk with antibiotic prophylaxis & & & & \\
\hline \multirow[t]{2}{*}{ All wound infections (SSSI + DSSI) } & Study population & & \multirow{2}{*}{$\begin{array}{l}\text { RR } 0.86 \\
(0.56 \text { to } 1.33)\end{array}$} & \multirow{2}{*}{$\begin{array}{l}1865 \\
\text { (5 RCTs) }\end{array}$} & \multirow{2}{*}{$\begin{array}{l}\oplus \odot \ominus \ominus \\
\text { VERY LOW } 12\end{array}$} & \\
\hline & 49 per 1000 & $\begin{array}{l}42 \text { per } 1000 \\
\text { ( } 27 \text { to } 65)\end{array}$ & & & & \\
\hline \multirow{2}{*}{$\begin{array}{l}\text { All wound infections (SSSI + DSSI) - low } \\
\text { infection risk environment }\end{array}$} & Study population & & \multirow{2}{*}{$\begin{array}{l}\text { RR } 0.63 \\
(0.28 \text { to } 1.41)\end{array}$} & \multirow{2}{*}{$\begin{array}{l}1302 \\
\text { (4 RCTs) }\end{array}$} & \multirow{2}{*}{$\begin{array}{l}\oplus \ominus \ominus \ominus \\
\text { VERY LOW } 23\end{array}$} & \\
\hline & 32 per 1000 & $\begin{array}{l}20 \text { per } 1000 \\
(9 \text { to } 45)\end{array}$ & & & & \\
\hline \multirow{2}{*}{$\begin{array}{l}\text { All wound infections (SSSI + DSSI) - high } \\
\text { infection risk environment }\end{array}$} & Study population & & \multirow{2}{*}{$\begin{array}{l}\text { RR } 0.99 \\
(0.58 \text { to } 1.68)\end{array}$} & \multirow{2}{*}{$\begin{array}{l}563 \\
(1 \mathrm{RCT})\end{array}$} & \multirow{2}{*}{$\begin{array}{l}\oplus \odot \odot \ominus \\
\text { VERY LOW } 24\end{array}$} & \\
\hline & 89 per 1000 & $\begin{array}{l}88 \text { per } 1000 \\
(52 \text { to } 150)\end{array}$ & & & & \\
\hline
\end{tabular}

${ }^{*}$ The risk in the intervention group (and its $95 \% \mathrm{Cl}$ ) is based on the assumed risk in the comparison group and the relative effect of the intervention (and its $95 \% \mathrm{Cl}$ ).

CI: Confidence interval; RR: Risk ratio; OR: Odds ratio

\section{GRADE Working Group grades of evidence}

High certainty: We are very confident that the true effect lies close to that of the estimate of the effect

Moderate certainty: We are moderately confident in the effect estimate: The true effect is likely to be close to the estimate of the effect, but there is a possibility that it is substantially different

Low certainty: Our confidence in the effect estimate is limited: The true effect may be substantially different from the estimate of the effect

Very low certainty: We have very little confidence in the effect estimate: The true effect is likely to be substantially different from the estimate of effect 
13 of 5 studies had high risk of bias for outcome or attrition bias.

2 The confidence interval of the pooled effect size estimate includes both benefit and harm. Also, the optimal information size was not reached and sample size was small. Downgrade -2

3 All studies have unclear or high risk of bias for selection bias, detection bias or attrition bias

4 The risk of bias for selection bias and detection bias is unclear and attrition bias is high for this study

Summary of findings 2 . Antibiotic prophylaxis compared to placebo for prevention of postoperative wound infection in adults undergoing open inguinal or femoral hernioplasty surgery

Antibiotic prophylaxis compared to placebo for prevention of postoperative wound infection in adults undergoing open elective inguinal or femoral hernioplasty hernia repair

\begin{tabular}{|c|c|c|c|c|c|c|}
\hline \multicolumn{7}{|l|}{$\begin{array}{l}\text { Patient or population: prevention of } \\
\text { Setting: } \\
\text { Intervention: antibiotic prophylaxis } \\
\text { Comparison: placebo }\end{array}$} \\
\hline \multirow[t]{2}{*}{ Outcomes } & \multicolumn{2}{|c|}{ Anticipated absolute effects* $(95 \% \mathrm{Cl})$} & \multirow{2}{*}{$\begin{array}{l}\text { Relative effect } \\
(95 \% \mathrm{Cl})\end{array}$} & \multirow{2}{*}{$\begin{array}{l}\text { № of partic- } \\
\text { ipants } \\
\text { (studies) }\end{array}$} & \multirow{2}{*}{$\begin{array}{l}\text { Certainty of the evi- } \\
\text { dence } \\
\text { (GRADE) }\end{array}$} & \multirow{2}{*}{$\begin{array}{l}\text { Com- } \\
\text { ments }\end{array}$} \\
\hline & Risk with placebo & Risk with antibiotic prophylaxis & & & & \\
\hline All wound infections (SSSI + DSSI) & 55 per 1000 & $\begin{array}{l}33 \text { per } 1000 \\
(26 \text { to } 43)\end{array}$ & $\begin{array}{l}\text { RR } 0.61 \\
(0.48 \text { to } 0.78)\end{array}$ & $\begin{array}{l}6443 \\
(22 \mathrm{RCTs})\end{array}$ & $\begin{array}{l}\oplus \oplus \oplus \ominus \\
\text { MODERATE } 1234\end{array}$ & \\
\hline \multirow{2}{*}{$\begin{array}{l}\text { All wound infections (SSSI + DSSI) - } \\
\text { low infection risk environment }\end{array}$} & Study population & & \multirow{2}{*}{$\begin{array}{l}\text { RR } 0.71 \\
(0.44 \text { to } 1.14)\end{array}$} & \multirow{2}{*}{$\begin{array}{l}3100 \\
\text { (9 RCTs) }\end{array}$} & \multirow{2}{*}{$\begin{array}{l}\oplus \oplus \oplus \odot \\
\text { MODERATE } 2356\end{array}$} & \\
\hline & 26 per 1000 & $\begin{array}{l}18 \text { per } 1000 \\
\text { (11 to } 30)\end{array}$ & & & & \\
\hline $\begin{array}{l}\text { All wound infections (SSSI + DSSI) - } \\
\text { high infection risk environment }\end{array}$ & 85 per 1000 & $\begin{array}{l}49 \text { per } 1000 \\
(37 \text { to } 65)\end{array}$ & $\begin{array}{l}\text { RR } 0.58 \\
(0.43 \text { to } 0.77)\end{array}$ & $\begin{array}{l}3343 \\
(13 \mathrm{RCTs})\end{array}$ & $\begin{array}{l}\oplus \odot \odot \ominus \\
\text { VERY LOW } 12367\end{array}$ & \\
\hline \multirow[t]{2}{*}{ SSSI } & Study population & & \multirow{2}{*}{$\begin{array}{l}\text { RR } 0.60 \\
(0.46 \text { to } 0.78)\end{array}$} & \multirow{2}{*}{$\begin{array}{l}6263 \\
(21 \mathrm{RCTs})\end{array}$} & \multirow{2}{*}{$\begin{array}{l}\oplus \oplus \oplus \odot \\
\text { MODERATE } 1234\end{array}$} & \\
\hline & 50 per 1000 & $\begin{array}{l}30 \text { per } 1000 \\
(23 \text { to } 39)\end{array}$ & & & & \\
\hline $\begin{array}{l}\text { SSSI - low infection risk environ- } \\
\text { ment }\end{array}$ & Study population & & $\begin{array}{l}\text { RR } 0.71 \\
\text { (0.44 to } 1.17 \text { ) }\end{array}$ & $\begin{array}{l}3100 \\
\text { (9 RCTs) }\end{array}$ & $\begin{array}{l}\oplus \oplus \oplus \odot \\
\text { MODERATE } 2356\end{array}$ & \\
\hline
\end{tabular}




\begin{tabular}{|c|c|c|c|c|c|}
\hline & 24 per 1000 & $\begin{array}{l}17 \text { per } 1000 \\
\text { (11 to } 28)\end{array}$ & & & \\
\hline \multirow{2}{*}{$\begin{array}{l}\text { SSSI - high infection risk environ- } \\
\text { ment }\end{array}$} & \multicolumn{2}{|c|}{ Study population } & \multirow{2}{*}{$\begin{array}{l}\text { RR } 0.56 \\
\text { (0.41 to } 0.77)\end{array}$} & \multirow{2}{*}{$\begin{array}{l}3163 \\
(12 \mathrm{RCTs})\end{array}$} & \multirow{2}{*}{$\begin{array}{l}\oplus \odot \odot \odot \\
\text { VERY LOW } 12367\end{array}$} \\
\hline & 79 per 1000 & $\begin{array}{l}44 \text { per } 1000 \\
(32 \text { to } 61)\end{array}$ & & & \\
\hline \multirow[t]{2}{*}{ DSSI } & \multicolumn{2}{|c|}{ Study population } & \multirow{2}{*}{$\begin{array}{l}\text { RR } 0.65 \\
(0.26 \text { to } 1.65)\end{array}$} & \multirow{2}{*}{$\begin{array}{l}4185 \\
(12 \mathrm{RCTs})\end{array}$} & \multirow{2}{*}{$\begin{array}{l}\oplus \oplus \oplus \odot \\
\text { MODERATE } 1234\end{array}$} \\
\hline & 6 per 1000 & $\begin{array}{l}4 \text { per } 1000 \\
(2 \text { to } 10)\end{array}$ & & & \\
\hline \multirow{2}{*}{$\begin{array}{l}\text { DSSI - low infection risk environ- } \\
\text { ment }\end{array}$} & \multicolumn{2}{|c|}{ Study population } & \multirow{2}{*}{$\begin{array}{l}\text { RR } 0.67 \\
(0.11 \text { to } 4.13 \text { ) }\end{array}$} & \multirow{2}{*}{$\begin{array}{l}1488 \\
\text { (3 RCTs) }\end{array}$} & \multirow{2}{*}{$\begin{array}{l}\oplus \oplus \oplus \odot \\
\text { MODERATE } 2356\end{array}$} \\
\hline & 4 per 1000 & $\begin{array}{l}3 \text { per } 1000 \\
(0 \text { to } 17)\end{array}$ & & & \\
\hline \multirow{2}{*}{$\begin{array}{l}\text { DSSI - high infection risk environ- } \\
\text { ment }\end{array}$} & \multicolumn{2}{|c|}{ Study population } & \multirow{2}{*}{$\begin{array}{l}\text { RR } 0.64 \\
(0.22 \text { to } 1.89)\end{array}$} & \multirow{2}{*}{$\begin{array}{l}2697 \\
\text { (9 RCTs) }\end{array}$} & \multirow{2}{*}{$\begin{array}{l}\oplus \oplus \odot \ominus \\
\text { LOW } 1236\end{array}$} \\
\hline & 7 per 1000 & $\begin{array}{l}4 \text { per } 1000 \\
(2 \text { to } 13)\end{array}$ & & & \\
\hline
\end{tabular}

${ }^{*}$ The risk in the intervention group (and its $95 \% \mathrm{Cl}$ ) is based on the assumed risk in the comparison group and the relative effect of the intervention (and its $95 \% \mathrm{Cl}$ ).

CI: Confidence interval; RR: Risk ratio; OR: Odds ratio

\section{GRADE Working Group grades of evidence}

High certainty: We are very confident that the true effect lies close to that of the estimate of the effect

Moderate certainty: We are moderately confident in the effect estimate: The true effect is likely to be close to the estimate of the effect, but there is a possibility that it is substantially different

Low certainty: Our confidence in the effect estimate is limited: The true effect may be substantially different from the estimate of the effect

Very low certainty: We have very little confidence in the effect estimate: The true effect is likely to be substantially different from the estimate of effect

1 Excluding the studies with unclear and/or high risk of bias from the meta-analysis affects the summary RR. Downgrade -1

${ }^{2} \mathrm{Chi}^{2}$ test $\mathrm{P}>0.05, \mathrm{I}^{2}<60 \%$, all confidence intervals of pooled effect estimates overlap. Clinical heterogeneity is limited as in similar patients are included, although different types and doses of antibiotics were applied. Evidence was not downgraded for clinical heterogeneity.

${ }^{3}$ There are no indirect comparisons or surrogate outcomes used in the studies

${ }^{4}$ The confidence interval of the pooled effect size includes both benefit and harm. Optimal information size was not reached, but evidence was not downgraded as the sample size is large.

${ }^{5}$ Excluding the studies with unclear or high risk of bias hardly affects the summary RR, therefore not downgraded.

${ }^{6}$ The confidence interval of the pooled effect size includes both benefit and harm. Optimal information size was not reached and sample size is small, therefore downgrade -1.

${ }^{7}$ Asymmetrical forest plot reveals a under representation of studies that favour the control treatment. 


\section{BACK G R O U N D}

\section{Description of the condition}

An inguinal hernia is a viscera or adipose tissue protrusion through the inguinal or femoral canal. The lifelong cumulative incidence of inguinal and femoral hernia repair in adults is $27 \%$ to $42.5 \%$ for men and $3 \%$ to $5.8 \%$ for women, and increases with age (HerniaSurge Group 2018; Fitzgibbons 2015).

Groin hernias are inguinal or femoral; inguinal hernias are either direct or indirect or a combination. Direct and indirect hernias both protrude above the inguinal ligament; a direct hernia protrudes medially to the inferior epigastric vessels, whereas an indirect hernia protrudes laterally (Gilbert 1989; Zollinger 2003; Fitzgibbons 2015). A femoral hernia protrudes below the inguinal ligament and medially to the femoral vessels (Fitzgibbons 2015). In day-to-day surgical practice a classification system of inguinal hernia types is seldom used other than the types described here (HerniaSurge Group 2018). Inguinal or femoral hernias can affect one or both sides of the groin, resulting in a unilateral or bilateral hernia respectively (Fitzgibbons 2015).

In the late 19th century Bassini described for the first time the pathophysiology of the groin hernia, namely a weakness of the posterior inguinal wall (Bassini 1890). He developed a hernia repair technique involving suturing the weakness of the posterior wall (herniorrhaphy) (Bassini 1890). His repair led to tension, however, and was associated with high recurrence rates and postoperative pain. Therefore, from the mid-20th century onwards surgeons began to experiment with synthetic meshes (Kurzer 1998). From the mid- and late-1980s tension-free, mesh-based (hernioplasty) methodologies were introduced for recurrent hernia and rapidly increased in popularity, as recurrence rates were lower, operation time was shorter, recovery time faster and surgery could be performed under local anaesthesia in a day care setting (Kurzer 1998; Robbins 1998). In 1989 Lichtenstein described the first large series (1000 participants) of mesh-based repair in primary hernia (Lichtenstein 1989).

Inguinal and femoral hernia repair is worldwide one of the most commonly performed surgical operations, with more than 20 million procedures annually, making up approximately a third of total surgical interventions (Cainzos 1990; Kingsnorth 2003; Rutkow 2003; Rodriguez 2005). A minority of patients are asymptomatic, but even a watch-and-wait approach in this group results in surgery in approximately $70 \%$ within seven years (Fitzgibbons 2013). In many countries hernioplasty probably remains the first choice and most frequently applied surgical method in a majority of cases, although accurate and recent data are not available (HerniaSurge Group 2018). Inguinal and femoral hernia repairs are considered clean surgical procedures with a low risk $(<5 \%)$ of postoperative wound infection (Ortega 2012; Cai 2018). However, when infectious complications do occur following inguinal or femoral hernia repair, they can be a risk factor for developing a recurrent hernia (Finan 2005). Development of a deep-mesh infection is rare, but is considered a serious complication as it usually requires mesh removal. Most studies report that (late) removal of the infected mesh usually does not result in hernia recurrence (Mann 1998; Fawole 2006; Johanet 2011; Rehman 2012).

postoperative wound infections can be divided into superficial surgical site infections (SSSI) and deep surgical site infections
(DSSI) (Mangram 1999; Mandell 2010). SSSI is defined by the Centers for Disease Control and Prevention (CDC) as an infection occurring within 30 days after the operation and affecting only the skin or subcutaneous tissue at the incision site. Additionally, at least one of the following characteristics should apply: (1) the infection is characterised by purulent drainage; (2) positive microbiological culture can be grown from fluids or tissue obtained from the incision; (3) pain, tenderness, swelling, redness or heat in the wound area occurs and re-opening of the wound by a surgeon is needed; (4) wound infection is diagnosed based on clinical experience of a surgeon of physician (Mangram 1999). DSSI is defined by the CDC as infection within one year after the operation if an implant is in place and the infection appears to be related to the operation. DSSI involves deep soft tissues (such as muscle or fascial layers). Additionally one of the following characteristics needs to be present: (1) purulent drainage from the deep incision; (2) spontaneous dehiscence or the wound is reopened by a surgeon for reasons of fever $\left(>38^{\circ} \mathrm{C}\right)$, localised pain or tenderness; (3) an abscess or infection is found on direct or radiologic or histopathologic examination; (4) wound infection is diagnosed based on clinical experience of a surgeon of physician (Mangram 1999).

Wound infections are caused by bacterial contamination during surgery or the postoperative period (Mangram 1999). Upon microbiological culture of the wound exudates, most often normal nasal or skin bacteria such as Staphylococcus aureus or Staphylococcus epidermidis bacterial strains are detected (Mangram 1999; Mandell 2010). Wound Infection may result from a number of factors both intrinsic and extrinsic to the patient (Mangram 1999). Several factors that may increase the infection rate after hernia repair have been analysed (Berard 1964; Haley 1985; Wittmann 1995; Porcu 1996; Finan 2005). Although many of these intrinsic factors (such as the overall clinical status of the patient) cannot be modified, the external ones (such as operation room ventilation, pre-operative shaving, length of operation time and experience of the surgeon) can certainly be influenced. In particular the factors related to aseptic conditions, surgical technique and perioperative care can be easily influenced (Mangram 1999). However, even under the most scrupulous aseptic conditions and with a careful technique, postoperative wound infection still presents a very serious problem. Antibiotic prophylaxis is therefore often used to decrease the risk of wound infections during surgical procedures (Mozillo 1988; Codina 1999; Heineck 1999; Mangram 1999; Mandell 2010; Bratzler 2013).

\section{Description of the intervention}

Antibiotic prophylaxis as an intervention to prevent surgical site infections and other infectious complications after surgery is recommended, especially in 'clean-contaminated', 'contaminated' and 'dirty-infected' surgical procedures (Mangram 1999; Bratzler 2013; Berrios-Torres 2017). For 'clean' surgery, such as inguinal and femoral hernia repair, antibiotic prophylaxis is not generally recommended (Mangram 1999; Berrios-Torres 2017). Antibiotic prophylaxis is recommended, however, when prosthetic material is being used or when risk factors are present (Condon 1991; Page 1993; Woods 1998; Mangram 1999; Simons 2009).

The most common surgical site infection pathogens after clean surgical procedures are Staphylococcus aureus or coagulasenegative Staphylococci (S. epidermidis) bacteria (Mangram 1999; Mandell 2010). S. epidermidis bacteria belong to commensal skin 
bacterial flora and S. aureus bacteria are often present on (nasal and respiratory tract) mucosal tissues as well as on skin (Mandell 2010). To a lesser extent bacteria belonging to the commensal intestinal bacterial flora, such as Escherichia coli, are detected in wound infection exudates (Mangram 1999; Mandell 2010; Bratzler 2013). Therefore antibiotics such as cefazolin or related cephalosporin class of antibiotics that kill these bacterial species are recommended for hernia repair (Mangram 1999; Mandell 2010; Bratzler 2013).

Severe adverse reactions to beta-lactam antibiotics are relatively rare (Lagace-Wiens 2012), but side effects such as diarrhoea, nausea/vomiting, rash, fatigue, itching and urticaria have more frequently been reported (Lagace-Wiens 2012). The cost of antibiotic prophylaxis is low, but much concern has been raised about the development of antibiotic-resistant bacteria when antibiotics are used too frequently or inappropriately (Mangram 1999; Mandell 2010; Chokshi 2019).

\section{How the intervention might work}

Different types of antibiotics, bactericidal and bacteriostatic, have different mechanisms of action but they respectively kill bacteria by interfering with bacterial cell wall synthesis or by preventing cell division by inhibiting protein synthesis (Mandell 2010).

The type of antibiotics used for prophylaxis during 'clean' surgical procedures belong to the beta-lactam bacteriocidal antibiotics type and more specifically to the cephalosporin-group (i.e. cefuroxime and cefazolin) or the penicillin-group (i.e. penicillin) antibiotics (Mandell 2010). These antibiotics are effective against bacterial species that are most often detected in infected surgical wounds( S. aureus, S. epidermidis or E. coli). Also, with a half-lifetime of 1 to 2 hours, a single dose of these antibiotics will supply a therapeutic level until approximately 3 to 7 hours after wound closure. (Mangram 1999; Bratzler 2013).

The use of antibiotic prophylaxis in a perioperative setting will reduce endogenous patient bacteria and bacteria that colonise patients' exposed tissues during surgery. Additionally, antibiotics could kill bacteria present on the implanted prosthetic material during hernioplasty surgery (Mandell 2010). Depending on the overall aseptic operative (room) conditions, poor conditions are likely to pose a higher risk for patients to develop surgical site infections (Mangram 1999; Mandell 2010). Altogether, antibiotic prophylaxis might prevent surgical site infections and other infectious complications.

\section{Why it is important to do this review}

Inguinal and femoral hernia repair are considered to be 'clean' surgical procedures, with an estimated postoperative wound infection rate of $1 \%$ to $5 \%$ (Mangram 1999; Ortega 2012; Bratzler 2013). Some studies have even estimated that the rate of postoperative infections should not be greater than $2 \%$ (Condon 1991; Page 1993; Rutkow 1993; Dellinger 1994; Woods 1998). In line with this, several randomised studies and systematic reviews reported wound infection rates after open hernia repair surgery ranging from $1.4 \%$ to $4.1 \%$ (Platt 1990; Morales 2000; Aufenacker 2004; Jain 2008; Cai 2018; Finch 2019). However, some randomised studies report wound infection rates exceeding $5 \%$ wound infection incidence with infection rates ranging up to $18.1 \%$ (Ullah 2013).
For suture-based inguinal and femoral hernia repair, antibiotic prophylaxis is not generally recommended in the absence of risk factors (Mangram 1999; Berrios-Torres 2017). Antibiotic prophylaxis is recommended for hernia repair involving the use of prosthetic material or when risk factors are present (Condon 1991; Page 1993; Woods 1998; Mangram 1999; Simons 2009; Berrios-Torres 2017). There is an ongoing debate whether antibiotic prophylaxis is of added value for preventing infectious complications when prosthetic materials are implanted during 'clean' surgical procedures (Stephenson 2003; Biswas 2005; Aufenacker 2006; Fawole 2006). Controversy especially arises when wound infection rates exceed the expected figures (Bailey 1992; Ranaboldo 1993; Holmes 1994; Wittmann 1995; Leaper 1998). Many parameters during surgery, such as length of operation, aseptic conditions, operation room ventilation, expertise of the surgeon etc., can affect postoperative wound infection rates. The effect of one of these surgery-related parameters, or the combined effect of more than one, results in the infection pressure for a specific clinical centre or study. The wound infection incidence in the control group is a reflection of the effect of single parameters combined and can therefore be regarded as a composite parameter for overall infection pressure. To address the issue of wound infection rates below or exceeding the expected $5 \%$ infection incidence for clean surgery, we stratified studies into high infection risk environments or low infection risk environments based on the wound infection incidence in the control group.

As with any medical drug treatment, antibiotic prophylaxis has side effects, including adverse reactions and allergic reactions. Moreover, the use of antibiotics can result in the development of antibiotic-resistant bacterial strains (Mandell 2010; Chokshi 2019). By reducing the use of unnecessary antibiotic prophylaxis, adverse events and development of antibiotic-resistant microbiological strains can be reduced.

\section{O B JECTIVES}

To determine the effectiveness of antibiotic prophylaxis in reducing postoperative (superficial and deep) wound infections in elective open inguinal and femoral hernia repair.

\section{METHODS}

\section{Criteria for considering studies for this review}

\section{Types of studies}

We included randomised controlled trials (RCTs) comparing antibiotic prophylaxis to placebo or no prophylaxis for open inguinal or femoral hernia repair surgery.

\section{Types of participants}

Adult male or female participants undergoing open elective inguinal or femoral hernia repair, with or without the use of prosthetic material. Studies for all types of inguinal or femoral hernia (unilateral, bilateral, primary or recurrent) were eligible for inclusion. We excluded studies addressing laparoscopic inguinal or femoral hernia repair surgery from this review.

\section{Types of interventions}

Studies comparing antibiotic prophylaxis of any type with placebo or no treatment in participants undergoing open inguinal or femoral hernia repair surgery. We also included studies that 
compared antibiotic prophylaxis against placebo for so-called 'clean wound' surgery in general, if data specifically for inguinal or femoral hernia could be extracted. We excluded studies in which antibiotic prophylaxis was compared with another antibiotic regimen, combinations of antibiotics, or studies comparing different doses or administration routes of antibiotics. We did not include studies using antiseptics of the incision area instead of antibiotic prophylaxis in the review.

\section{Types of outcome measures}

\section{Primary outcomes}

Wound infection after inguinal or femoral repair surgery. We classified wound infections as superficial surgical site infections (SSSI), deep surgical site infections (DSSI) or all wound infections (a sum total of SSSI and DSSI). SSSI can occur up to 30 days postoperatively and DSSI can occur up to one year postoperatively (Mangram 1999). We included studies reporting wound infections as defined by Centers for Disease Control and Prevention (CDC) (Horan 1992; Berrios-Torres 2017); or as defined specifically by authors (definitions including parameters such as discharge of pus from the wound; a wound that was opened and not closed; spreading erythema indicative of cellulitis). We excluded other postoperative infections (i.e. pulmonary, urinary tract and other infections) from analysis.

\section{Secondary outcomes}

Any adverse event attributable to the use of antibiotics, including allergic reactions of participants. We accepted any definition of adverse event by the primary authors. We also analysed the reporting of development of antibiotic resistance due to antibiotic prophylaxis.

\section{Search methods for identification of studies}

\section{Electronic searches}

For this update we searched the following electronic databases, with no restriction on language or publication date, on 12 November 2019.

1. Cochrane Registry of Studies Online (CRSO.cochrane.org) (Appendix 1)

2. MEDLINE Ovid (1946 to 12 November 2019) (Appendix 2)

3. Embase Ovid (1946 to 12 November 2019) (Appendix 3)

4. Scopus (2011 to 2019) (Appendix 4)

5. Science Citation Index (Web of Science) (2011 to 2019) (Appendix 5)

The MEDLINE search strategy that was used in previous versions of this review can be found in Appendix 6. In the previous updates, the searches were performed until June 2009 and October 2011.

\section{Searching other resources}

We contacted the Dutch inguinal hernia guideline panel of medical specialists for information about any additional completed studies. We checked the reference lists of the included studies to identify further studies.

We searched prospective trial registers with the following search terms: (inguinal hernia OR groin hernia) AND (antibiotic OR antibiotics) (search date 12 November 2019).
1. ClinicalTrials.gov

2. World Health Organization (WHO) International Clinical Trials Registry Platform (ICTRP) (www.who.int/ictrp/en)

\section{Data collection and analysis}

\section{Selection of studies}

Two review authors ( $\mathrm{CO}$ and $\mathrm{CvH}$ ) independently assessed all titles and abstracts that were retrieved in the literature search in Covidence software. We included studies when study design, intervention and type of participants met the inclusion criteria for this review. We resolved disagreements on the inclusion or exclusion of studies by discussion.

\section{Data extraction and management}

Two authors (CO and FJSM) developed a digital data extraction form, which was piloted by two authors ( $\mathrm{CO}$ and $\mathrm{CvH}$ ) on three studies to ensure that data extraction was performed in a consistent manner. Data extraction was performed by pairs of authors (from $\mathrm{CO}, \mathrm{CvH}$ and FJSM) independently. We resolved disagreements by discussion or by involving another author (RS) as arbiter. When we considered a publication to be eligible but data in the publication were incomplete, we contacted the principal author to obtain the necessary information. We subsequently entered extracted data into Review Manager 5 (RevMan 5) software (Review Manager 2014).

We extracted the following data.

1. General study information, including first author and year of publication.

2. Study methods, including study design, country and enrolment period of the participants.

3. Participant characteristics, including age and sex distribution, body mass index (BMI), American Society of Anesthesiologists (ASA) status of the participants, inclusion and exclusion criteria, and specific information on the surgical or hospitalisation procedure (including method of repair, skin antiseptic, operative time, type of anaesthesia, drain use, surgeons' expertise, hospitalisation method and length of stay).

4. Intervention information, including type of antibiotic (or placebo), dose, number of doses, administration route, and administration timing.

5. Outcome information, including the wound infection definition that was applied, duration of follow-up, number of participants with a wound infection per total number of participants in both the intervention (antibiotic prophylaxis) and control (placebo) group for any superficial or deep surgical wound infection (SSSI, DSSI).

6. Other relevant information, including details on which bacterial species were cultured from the infection sites or adverse effects.

\section{Assessment of risk of bias in included studies}

Pairs of review authors ( $\mathrm{CO}$ and FJSM or $\mathrm{CO}$ and $\mathrm{CvH}$ ) independently assessed the risk of bias for each included study. We resolved disagreements by discussion or by involvement of an independent author (RS) as arbiter. We assessed all included studies for risk of bias based on the criteria as described in Chapter 8 of the Cochrane Handbook for Systematic Reviews of Interventions (version 5.2.0) (Higgins 2017). 
We assessed the following risk of bias domains: random sequence generation (selection bias); allocation concealment (selection bias); blinding of participants and personnel (performance bias); blinding of outcome assessment (detection bias); incomplete outcome data (attrition bias); selective reporting (reporting bias); and other bias. In addition to the assessment criteria described in Chapter 8 of the Cochrane Handbook for Systematic Reviews of Interventions (version 5.2.0) (Higgins 2017), we judged random sequence generation as low risk of bias when block randomisation was reported, as block randomisation indicates that authors have considered the importance of randomisation to prevent selection bias. We judged studies that reported that allocation concealment involved (numbered) sealed envelopes as unclear bias as the information on sequentially numbered envelopes and opaqueness was not reported. We judged studies that were terminated early after interim analysis of the results as high risk of bias for the domain 'other bias'.

We considered a study at low risk of bias if we assessed the domains 'selection bias', 'performance bias' and 'detection bias' as low risk and none of the other domains was at high risk. We contacted authors when there were any questions regarding risk of bias in their publication. If we did not succeed in retrieving additional information from study authors we had to make our final judgement of methodological quality on the basis of the available information.

\section{Measures of treatment effect}

We analysed the data using Cochrane Review Manager 5 software (Review Manager 2014). We used a Mantel-Haenszel random-effects model to compute risk ratios (RRs) with $95 \%$ confidence intervals (Cls) for dichotomous outcomes.

\section{Unit of analysis issues}

We used the number of individual participants as the unit of analysis. All trials were parallel in design. We combined participants from both intervention groups for meta-analyses that included studies that compared more than one intervention with the same control group.

\section{Dealing with missing data}

For each study we extracted the number of participants originally allocated to each treatment group and performed intention-totreat analysis, or available-case analysis when participants were lost to follow-up.

\section{Assessment of heterogeneity}

We assessed clinical diversity among the included studies by analysing differences in participants and interventions, as well as outcome measurements and length of follow-up period. We first performed a visual inspection of forest plots. Then we used both the $\mathrm{Chi}^{2}$ test for heterogeneity and the $\mathrm{I}^{2}$ statistic to test for heterogeneity between study results. We set the $\mathrm{P}$ value for the $\mathrm{Chi}^{2}$ test at a conservative 0.1 ; and we considered $I^{2}$ values of $50 \%$ to $90 \%$ as indicating substantial heterogeneity.

\section{Assessment of reporting biases}

We obtained fewer than 10 studies for the meta-analysis of herniorrhaphy repair studies, so assessment of reporting bias by funnel plot analysis was not possible. We performed a funnel plot analysis for hernioplasty groin hernia repair studies for the outcomes 'all wound infection' (SSSI + DSSI) and for 'superficial surgical site infections' (SSSI) for both infection risk subgroups.

\section{Data synthesis}

We pooled dichotomous outcomes from studies by meta-analysis and used a random-effects model to analyse data. We gathered results from herniorrhaphy studies separately from hernioplasty studies. We used Review Manager 5 to generate forest plots and additionally summarised results narratively (Review Manager 2014). We used GRADEpro software to summarise findings and to present the quality of evidence in the 'Summary of findings' tables (GRADE pro GDT).

\section{Subgroup analysis and investigation of heterogeneity}

We performed separate analyses for the various categories of surgery that were performed (i.e. herniorrhaphies or hernioplasties). Inguinal or femoral hernia repair is considered a clean surgical procedure with wound infection rates of $5 \%$ or lower (Mangram 1999; Ortega 2012). A number of studies reported wound infection rates that exceeded the generally accepted rate. To address this issue, we stratified studies into high infection risk environments ( $\geq 5 \%$ infection incidence) or low infection risk environments ( $<5 \%$ infection incidence) based on the wound infection incidence in the control group. Many parameters during surgery, such as length of operation, aseptic conditions, operation room ventilation, expertise of the surgeon etc. can affect postoperative wound infection rates. The effect of one or a combination of these single surgical-related parameters results in the observed infection pressure for a specific clinical centre or study. The wound infection incidence in the control group is a reflection of the effect of single parameters combined and can therefore be regarded as a composite parameter for overall infection pressure.

\section{Sensitivity analysis}

We performed sensitivity analysis by excluding studies at high risk of bias or studies with more than $10 \%$ missing outcome data from the meta-analysis.

\section{RESULTS}

\section{Description of studies}

\section{Results of the search}

We identified 430 studies by searching the primary electronic databases (140 in MEDLINE, 78 in Embase and 85 in the Cochrane Registry of Studies Online; the remaining studies in Scopus and Web of Science). After removal of duplicates, 285 studies were left. We screened the titles and abstracts of these studies, and selected 46 for full-text review. Reasons for excluding studies are provided In Characteristics of excluded studies. We finally included 27 studies, reported in 28 publications, that met the inclusion criteria for both the qualitative and quantitative synthesis (meta-analysis). Lazorthes 1992 was reported in two publications (one in French and one in English), hence we discarded Lazorthes 1993. We present the PRISMA study flow chart in Figure 1. 
Figure 1. Study flow diagram.

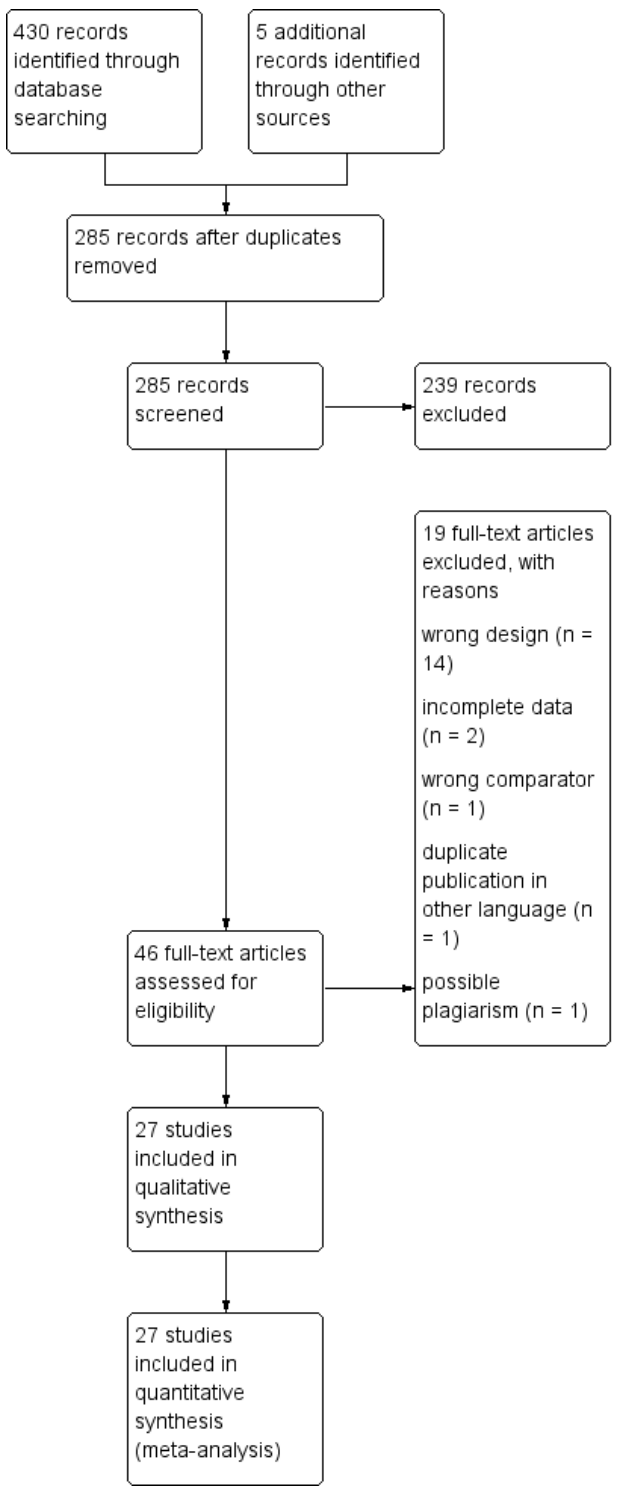


Seventeen RCTs were included in the meta-analyses in the previous version of this review (Sanchez-Manuel 2012).

We searched two prospective trial registers and retrieved five study protocols corresponding to four publications (Ergul 2011; Rahmani 2012; Mazaki 2013; Wang 2013). Additionally, we retrieved one relevant study protocol of which no publication was found. We have added this study to the Characteristics of studies awaiting classification.

\section{Included studies}

We present detailed information of the 27 included studies in Characteristics of included studies. Of those, 24 were published in English; two in Spanish (Morales 2000; Oteiza 2004); and one in Farsi (Rahmani 2012).

Five RCTs used herniorrhaphy as surgical procedure (Evans 1973; Andersen 1980; Platt 1990; Lazorthes 1992; Taylor 1997); and 22 RCTs used hernioplasty as surgical procedure for inguinal or femoral hernia repair. The studies using herniorrhaphy surgical procedure were published in the period 1973 to 1997, while the studies applying hernioplasty surgical procedures were published in the period from 2004 to 2015 .

We included a total of 8308 participants in this systematic review, of whom 1865 underwent herniorrhaphy surgery. Within this group, 922 participants received antibiotic prophylaxis and 943 received either no antibiotics or placebo. In the hernioplasty group 6443 participants were included; 3399 received antibiotic prophylaxis and 3044 received either no antibiotics or placebo.

\section{Treatment characteristics of the studies}

The treatment characteristics of the included herniorrhaphy studies are described in Table 1 and those of the included hernioplasty studies in Table 2. Further details are described in Characteristics of included studies.

In the herniorrhaphy studies, all studies used different antibiotics as prophylaxis (Table 1 ). In the hernioplasty studies (Table 2) the two antibiotics that were most frequently applied were amoxicillinclavulanic acid (AMC; Oteiza 2004; Jain 2008; Al-Fatah 2011; Goyal 2011; Othman 2011; Ullah 2013; Kochhar 2014); and cefazolin (CZO; Morales 2000; Celdran 2004; Perez 2005; Ijaz 2010; Shankar 2010; Thakur 2010; Ergul 2011; Mazaki 2013; Wang 2013; Razack 2015). Other applied antibiotics are summarised in Table 2. One study compared two different antibiotics against placebo (Wang 2013). We combined results from both antibiotic prophylaxis intervention groups in the meta-analysis.

All four studies administered antibiotic prophylaxis intravenously. Andersen 1980 applied antibiotics subfascially before closure; Evans 1973 applied the first dose of antibiotics intravenously, but the second and third dose intramuscularly; Lazorthes 1992 added antibiotics to local anaesthesia solution; and Ullah 2013 did not specify the route of administration.

The follow-up period in the herniorrhaphy studies ranged from one week in Platt 1990 to four to six weeks (Evans 1973; Andersen 1980; Platt 1990; Lazorthes 1992; Taylor 1997). Only one study had a follow-up of one year to investigate hernia recurrences (Andersen 1980). The follow-up period reported in the hernioplasty studies ranged from several days or one week postoperatively to two to six weeks. A limited number of studies had follow-up time points at three months (Aufenacker 2004; Celdran 2004; Mazaki 2013), six months (Yerdel 2001; Celdran 2004) or one year (Morales 2000; Celdran 2004; Jain 2008). Goyal 2011 was the only study with a single follow-up time point at one week postoperatively.

Four herniorrhaphy studies did not report the length of operative time (Evans 1973; Andersen 1980; Lazorthes 1992; Taylor 1997); nor did seven hernioplasty studies (Morales 2000; Ijaz 2010; Goyal 2011; Rahmani 2012; Ullah 2013; Wang 2013; Kochhar 2014). The remaining herniorrhaphy study reported a mean operation length of more than one hour (Platt 1990). Eleven hernioplasty studies reported a mean (or median) length of operative time equal to or less than one hour (Aufenacker 2004; Oteiza 2004; Perez 2005; Tzovaras 2007; Jain 2008; Shankar 2010; Al-Fatah 2011; Ergul 2011; Othman 2011; Bidhur 2013; Razack 2015); and three studies reported a mean (or median) of more than one hour (Yerdel 2001; Celdran 2004; Mazaki 2013), with Thakur 2010 reporting that $79 \%$ to $92 \%$ of the operations had a total duration of more than one hour.

Three studies investigated the effectiveness of antibiotic prophylaxis for the prevention of wound infections not only in inguinal or femoral hernia repair, but also in several other types of surgical procedures: cholecystectomy (Andersen 1980); clean and contaminated surgery (Evans 1973); and breast surgery (Platt 1990).

Wound infection rates in the control group (without antibiotic prophylaxis) ranged widely, from $0 \%$ to $18.1 \%$. Nine studies were considered low infection risk environments (Morales 2000; Aufenacker 2004; Oteiza 2004; Perez 2005; Tzovaras 2007; Jain 2008; Goyal 2011; Bidhur 2013; Kochhar 2014); and 13 studies were considered high-risk environments (Yerdel 2001; Celdran 2004; Ijaz 2010; Shankar 2010; Thakur 2010; Al-Fatah 2011; Ergul 2011; Othman 2011; Rahmani 2012; Mazaki 2013; Ullah 2013; Wang 2013; Razack 2015).

\section{Participant characteristics}

The participant characteristics of the included herniorrhaphy studies are described in Table 3 and of the included hernioplasty studies in Table 4. Further details are described in Characteristics of included studies.

Most study participants were male (ranging from $72 \%$ to $99 \%$ ), with three studies including only males (Jain 2008; Thakur 2010; Ullah 2013). The only exception was the study of Rahmani 2012, that included $41 \%$ to $45 \%$ males.

Mean (or median) age in the herniorrhaphy studies ranged from 50 to 51 years (Platt 1990) and from 62 to 70 years (Lazorthes 1992). Age of the participants was not separately reported for the hernia repair group in one study (Evans 1973). In the hernioplasty studies the mean or median age ranged from younger than 50 years (Jain 2008; Ijaz 2010; Shankar 2010; Ergul 2011; Othman 2011; Bidhur 2013; Kochhar 2014; Razack 2015) up to 72 years (Mazaki 2013). Thakur 2010 did not report any age characteristics of participants. Nine studies also included participants aged younger than 18 years (Lazorthes 1992; Oteiza 2004; Tzovaras 2007; Shankar 2010; AlFatah 2011; Goyal 2011; Wang 2013; Kochhar 2014; Razack 2015).

Known risk factors for wound infection development (Finan 2005; Mandell 2010), such as high American Society of Anesthesiologists (ASA) score or high body mass index (BMI) of participants were not consistently reported. Herniorrhaphy studies did not report 
these characteristics, except the study of Platt and colleagues that reported the BMI of the participants (Platt 1990).

Of the hernioplasty studies, nine did not report any ASA score information (Aufenacker 2004; ljaz 2010; Thakur 2010; Goyal 2011; Othman 2011; Rahmani 2012; Bidhur 2013; Ullah 2013; Kochhar 2014); and 16 did not report BMI information (Morales 2000; Aufenacker 2004; Oteiza 2004; Perez 2005; Jain 2008; ljaz 2010; Shankar 2010; Thakur 2010; Ergul 2011; Goyal 2011; Othman 2011; Rahmani 2012; Bidhur 2013; Ullah 2013; Kochhar 2014; Razack 2015). Most studies included participants with a low (I/II) ASA score (Yerdel 2001; Celdran 2004; Perez 2005; Jain 2008; Shankar 2010; Razack 2015), few studies also included ASA III score participants (Morales 2000; Tzovaras 2007; Al-Fatah 2011; Mazaki 2013) or ASA IV score participants (Ergul 2011). Wang 2013 did not specifically address the score, but reported that participants with an ASA score of greater than 1 were included in their study.

The BMI in the studies ranged from $23 \mathrm{~kg} / \mathrm{m}^{2}$ to $26 \mathrm{~kg} / \mathrm{m}^{2}$ (Yerdel 2001; Celdran 2004; Tzovaras 2007; Al-Fatah 2011; Mazaki 2013), with the exception of Wang 2013 in which a range of $18 \mathrm{~kg} / \mathrm{m}^{2}$ to 32 $\mathrm{kg} / \mathrm{m}^{2}$ was reported.

\section{Excluded studies}

Studies were excluded for the following reasons (see also Characteristics of excluded studies).
1. Studies focused on clean surgical techniques including inguinal or femoral hernia pathology, but data for this subgroup of patients could not be collected (Esposito 2006; Mehrabi Bahar 2015).

2. Study design not eligible: Gierhake 1975, Taylor 1996, Barreca 2000, Sanchez-Manuel 2003, Aufenacker 2006, Pessaux 2006 (prognostic study based on three randomised studies that compared antibiotics prophylaxis versus another antibiotic prophylaxis regime), Sanabria 2007, Leon 2011 (nonrandomised study), Li 2012; Sanchez-Manuel 2012, Yin 2012 (systematic reviews), Mazaki 2013b, Erdas 2016, Boonchan 2017.

3. Comparator not eligible (antibiotics prophylaxis versus antibiotics prophylaxis with another regime): Pessaux 2006, Bhuiyan 2017.

4. Article in French (Lazorthes 1993) of similar study described previously in English (Lazorthes 1992)

5. The results section and large text fragments of the study of Ahmed 2014 were similar to those of the study of Goyal 2011. We therefore considered that Ahmed 2014 was plagiarised and excluded it from analysis.

\section{Risk of bias in included studies}

A summary of the risk of bias is presented in Figure 2. Detailed information on risk of bias assessment, including substantiation for all risk of bias categories for each individual study can be found in the 'Risk of bias' tables of the Characteristics of included studies. 
Figure 2.

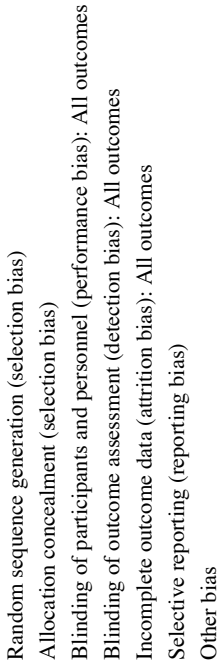

Al-Fatah 2011

Andersen 1980

\begin{tabular}{|l|l|l|l|l|l|l|}
\hline+ & $?$ & + & + & + & + & + \\
\hline
\end{tabular}

Aufenacker 2004

\begin{tabular}{lllllll}
\hline$?$ & $?$ & $\oplus$ & $?$ & + & + & + \\
\hline
\end{tabular}

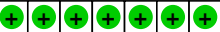

Bidhur 2013

+ †

Celdran 2004

$\hookrightarrow$ ? $+\uparrow \oplus+\odot$

Ergul 2011

$\hookrightarrow$ ? $+\uparrow+\oplus$

Evans 1973

+ ? $+\odot \odot \oplus$

Goyal 2011

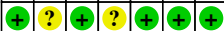

Ijaz 2010

\begin{tabular}{lllllll}
\hline$?$ & $?$ & + & $?$ & + & + & +
\end{tabular}

Jain 2008

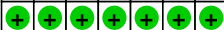

Kochhar 2014

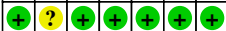

Lazorthes 1992

Mazaki 2013

Morales 2000

$\begin{array}{llllllll}? & \text { ? } & + & \text { ? } & + & + & +\end{array}$

Oteiza 2004

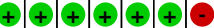

Othman 2011

$+\oplus+\odot$ ? +

Perez 2005

+ ? $+\odot \oplus+\oplus$

Platt 1990

$++\odot$ ? $+\oplus$

Platt 1990

$+++++$

Rahmani 2012

Razack 2015

Shankar 2010

Taylor 1997

Thakur 2010

+ ? $++\odot+$

\begin{tabular}{lllllll}
\hline$?$ & $?$ & + & $?$ & + & $\odot$ & + \\
\hline
\end{tabular}

\begin{tabular}{llllllll}
\hline$?$ & $?$ & + & + & - & $?$ & + \\
\hline
\end{tabular}

? $?++\odot \oplus+$

\begin{tabular}{lllllll}
\hline & $?$ & + & $?$ & $\odot$ & + & +
\end{tabular}

Thovaras 2007

+ ? $+\odot$ ?

Ullah 2013

Wang 2013

? ++++

Yerdel 2001

$\hookrightarrow$ ? + ? $+\oplus$

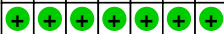

$++++\leftarrow+$ 


\section{Allocation}

Six studies provided no (or insufficient) information on random sequence generation (Andersen 1980; Lazorthes 1992; ljaz 2010; Shankar 2010; Rahmani 2012; Razack 2015) and we therefore judged them at 'unclear risk of bias'. Allocation concealment was insufficiently reported in the majority (19 of 27) of studies and we therefore scored them as 'unclear risk of bias'. Eight studies scored 'low risk of bias' for both random sequence generation and allocation concealment (Morales 2000; Yerdel 2001; Aufenacker 2004; Perez 2005; Jain 2008; Othman 2011; Mazaki 2013; Wang 2013).

\section{Blinding}

Twelve studies reported the blinding for participants and personnel (Platt 1990; Morales 2000; Yerdel 2001; Aufenacker 2004; Perez 2005; Tzovaras 2007; Jain 2008; Al-Fatah 2011; Ergul 2011; Othman 2011; Mazaki 2013; Wang 2013). The other 15 studies provided no or insufficient information on participant or personnel blinding, or both. However, we scored all studies as 'low risk of bias' as we judged that participant and personnel blinding in this setting was unlikely to contribute to performance bias.

In contrast to participant and personnel blinding, the blinding of outcome assessment is pivotal in this setting as there is a subjective element in the assessment of wound infection. Most studies refer to Centers for Disease Control and Prevention (CDC) wound infection criteria or alternatively specifically describe wound infection criteria. Ten studies scored an 'unclear risk of bias' for detection bias (Andersen 1980; Lazorthes 1992; Taylor 1997; Ijaz 2010; Thakur 2010; Goyal 2011; Othman 2011; Rahmani 2012; Bidhur 2013; Ullah 2013), as these studies did not provide (specific) information about the person who performed outcome assessment. The study by Evans and colleagues scored 'high risk of bias' as surgeons who performed procedures were also involved in outcome assessment while blinding of personnel was insufficiently reported (Evans 1973).

\section{Incomplete outcome data}

There were no missing data in 13 studies (Evans 1973; Celdran 2004; Perez 2005; Jain 2008; ljaz 2010; Thakur 2010; Al-Fatah 2011; Ergul 2011; Goyal 2011; Othman 2011; Rahmani 2012; Bidhur 2013; Ullah 2013). The other studies reported low numbers of missing data, and missing data were also balanced between treatment groups (Andersen 1980; Lazorthes 1992; Morales 2000; Yerdel 2001; Aufenacker 2004; Oteiza 2004; Tzovaras 2007; Mazaki 2013; Wang 2013; Kochhar 2014). These studies performed available-case analysis on their data.
We scored five studies as 'high risk' for attrition bias. In the study by Evans and colleagues, it was reported that patients died from unexplained reasons after surgery and were excluded from analysis (Evans 1973). In the other four studies high numbers of missing data were reported ranging from 7.7\% (Platt 1990), 9\% (Taylor 1997), $10 \%$ (Razack 2015), up to $26 \%$ (Shankar 2010), and missing data were not balanced between treatment groups (Razack 2015).

\section{Selective reporting}

Protocol registration was not reported in the herniorrhaphy studies. The reported outcome in the herniorrhaphy studies was wound infection, without further distinction between superficial or deep surgical site infections.

Three hernioplasty studies prospectively registered a study protocol and we scored them at 'low risk of bias' (Ergul 2011; Mazaki 2013; Wang 2013). One study registered the study protocol after the study was conducted and we therefore scored it at 'high risk of reporting bias' (Rahmani 2012). No other hernioplasty studies registered a study protocol. Most studies did, however, provide data for expected outcomes (superficial and deep surgical site infection: SSSI and DSSI). Eleven studies reported both outcomes separately (Yerdel 2001; Aufenacker 2004; Celdran 2004; Perez 2005; Shankar 2010; Thakur 2010; Ergul 2011; Othman 2011; Rahmani 2012; Mazaki 2013; Wang 2013); and nine studies reported only SSSI (Oteiza 2004; Tzovaras 2007; Jain 2008; Ijaz 2010; Al-Fatah 2011; Goyal 2011; Bidhur 2013; Ullah 2013; Kochhar 2014). We rated these studies as 'low risk of bias'.

Two hernioplasty studies did not distinguish between SSSI and DSSI outcomes and therefore meta-analysis for either outcome was not possible (Morales 2000; Razack 2015). For this reason we scored these studies as 'unclear risk of bias'.

\section{Other potential sources of bias}

Three studies were terminated early after interim analysis of the results (Yerdel 2001; Celdran 2004; Mazaki 2013). As early study termination could have introduced risk of bias for increased benefit in the antibiotic prophylaxis group, these three studies were scored 'high risk' for 'other bias'.

\section{Effects of interventions}

See: Summary of findings 1 Antibiotic prophylaxis compared to placebo for prevention of postoperative wound infection in adults undergoing open inguinal or femoral herniorrhaphy surgery; Summary of findings 2 Antibiotic prophylaxis compared to placebo for prevention of postoperative wound infection in adults undergoing open inguinal or femoral hernioplasty surgery 


\section{Primary outcomes}

\subsection{Herniorrhaphy repair}

The overall wound infection incidence for the herniorrhaphy studies is $4.3 \%$, with $3.8 \%$ in the antibiotic prophylaxis group and $4.9 \%$ in the control group. The incidence of wound infections in the control groups in individual herniorrhaphy repair surgery studies ranged from $0 \%$ to $8.9 \%$. In the low infection risk environment studies the wound infection incidence ranged from $0 \%$ to $4.6 \%$ and in the high infection risk environment the infection incidence was $8.9 \%$.

All five studies reported the number of participants who developed a wound infection of any type after herniorrhaphy surgery for inguinal or femoral hernia repair (Figure 3) (Evans 1973; Andersen
1980; Platt 1990; Lazorthes 1992; Taylor 1997). No distinction was made between superficial or deep surgical site infections and therefore we could not analyse these outcomes separately. Overall, 35 participants in the antibiotic prophylaxis group developed a wound infection and 25 participants in the placebo/control group. The summary risk ratio (RR) for wound infection of antibiotic prophylaxis compared with placebo or no prophylaxis was 0.86 (95\% confidence interval ( $\mathrm{Cl}) 0.56$ to 1.33 ). There were no signs of heterogeneity $\left(\mathrm{Chi}^{2} \mathrm{P}=0.41\right.$ and $\left.\mathrm{I}^{2}=0 \%\right)$. For a mean background risk in the control group of $4.9 \%$, the number needed to treat for an additional beneficial outcome (NNTB) is 146. In the low risk environment group $(<5 \%$ infections in the control group; four studies with 1302 participants) the RR for any wound infection was $0.63(95 \% \mathrm{Cl} 0.28$ to 1.41$)$ and in the high risk environment group (one study with 563 participants) the RR was 0.99 (95\% Cl 0.58 to 1.68). The $P$ value of the test for subgroup differences was 0.85 .

Figure 3. Forest plot of comparison antibiotic prophylaxis against placebo for preventing all types of wound infection (SSSI and DSSI) after herniorrhaphy surgery.

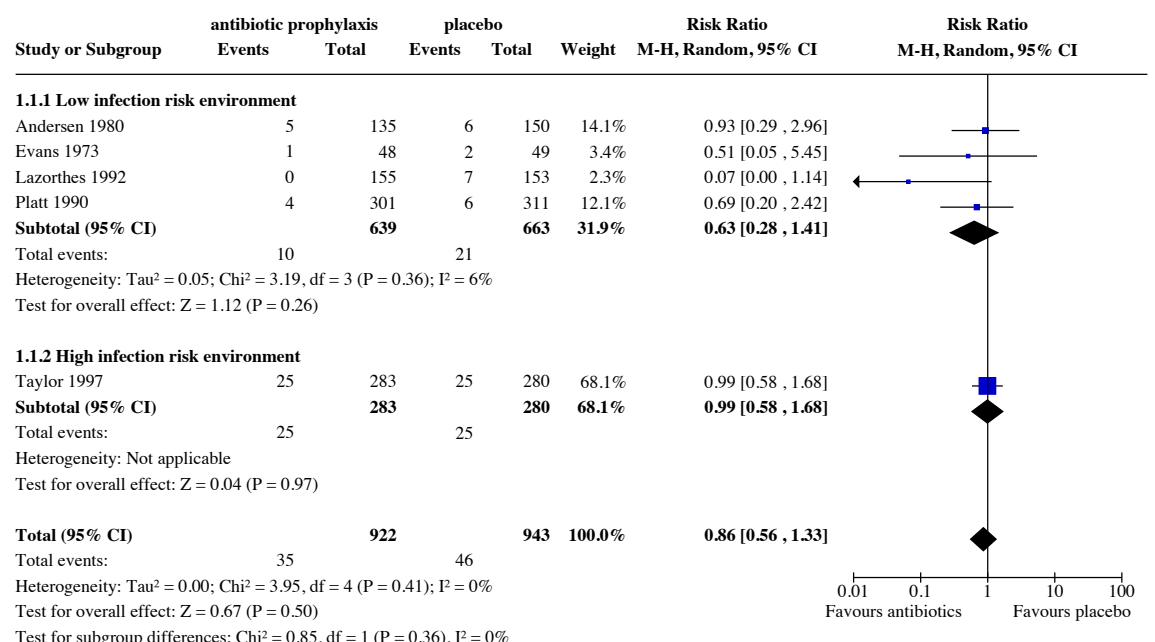

All herniorrhaphy studies scored at least two 'unclear' or 'high' risk of bias items and therefore we could not perform a sensitivity analysis.

\subsection{Hernioplasty repair}

\subsubsection{All wound infections (SSSI + DSSI)}

Twenty-two studies reported on all wound infections (SSSI + DSSI) in all risk environments with a total of 109 wound infections in the antibiotic prophylaxis group and 166 wound infections in the control group (Figure 4). The average wound infection incidence is $4.2 \%$, with $3.2 \%$ in the antibiotic prophylaxis group and $5.5 \%$ in the control group. The incidence of wound infections in the control groups of the individual hernioplasty repair surgery studies ranged from $0 \%$ to $18.1 \%$. In the low infection risk environment studies the wound infection incidence in the control groups ranged from $0 \%$ to $4.8 \%$ and in the high infection risk environment the infection incidence in the control groups ranged from $5.0 \%$ to $18.1 \%$. 
Figure 4. Forest plot of comparison antibiotic prophylaxis against placebo for preventing all types of wound infection (SSSI and DSSI) after hernioplasty surgery.

\begin{tabular}{|c|c|c|c|c|c|c|c|}
\hline \multirow[b]{2}{*}{ Study or Subgroup } & \multicolumn{2}{|c|}{ Prophylaxis } & \multicolumn{2}{|c|}{ Control } & \multicolumn{2}{|r|}{ Risk Ratio } & Risk Ratio \\
\hline & Events & Total & Events & Total & Weight & M-H, Random, $95 \%$ CI & M-H, Random, $95 \%$ CI \\
\hline
\end{tabular}

\begin{tabular}{|c|c|c|c|c|c|}
\hline \multicolumn{6}{|c|}{ 2.1.1 low infection risk environment } \\
\hline Aufenacker 2004 & 8 & 503 & 9 & 505 & $6.8 \%$ \\
\hline Bidhur 2013 & 0 & 30 & 1 & 30 & $0.6 \%$ \\
\hline Goyal 2011 & 1 & 100 & 3 & 100 & $1.2 \%$ \\
\hline Jain 2008 & 1 & 60 & 1 & 60 & $0.8 \%$ \\
\hline Kochhar 2014 & 4 & 106 & 5 & 106 & $3.6 \%$ \\
\hline Morales 2000 & 4 & 233 & 6 & 281 & $3.8 \%$ \\
\hline Oteiza 2004 & 1 & 124 & 0 & 123 & $0.6 \%$ \\
\hline Perez 2005 & 4 & 180 & 7 & 180 & $4.1 \%$ \\
\hline Tzovaras 2007 & 5 & 190 & 9 & 189 & $5.2 \%$ \\
\hline Subtotal (95\% CI) & & 1526 & & 1574 & $26.7 \%$ \\
\hline Total events: & 28 & & 41 & & \\
\hline \multicolumn{6}{|c|}{ Heterogeneity: $\mathrm{Tau}^{2}=0.00 ; \mathrm{Chi}^{2}=2.12, \mathrm{df}=8(\mathrm{P}=0.98) ; \mathrm{I}^{2}=0 \%$} \\
\hline \multicolumn{6}{|c|}{ Test for overall effect: $\mathrm{Z}=1.41(\mathrm{P}=0.16)$} \\
\hline \multicolumn{6}{|c|}{ 2.1.2 high infection risk environment } \\
\hline Al-Fatah 2011 & 3 & 100 & 5 & 100 & $3.1 \%$ \\
\hline Celdran 2004 & 0 & 50 & 4 & 49 & $0.7 \%$ \\
\hline Ergul 2011 & 5 & 100 & 7 & 100 & $4.9 \%$ \\
\hline Ijaz 2010 & 2 & 50 & 5 & 50 & $2.4 \%$ \\
\hline Mazaki 2013 & 2 & 100 & 13 & 100 & $2.8 \%$ \\
\hline Othman 2011 & 4 & 50 & 6 & 48 & $4.2 \%$ \\
\hline Rahmani 2012 & 4 & 141 & 9 & 141 & $4.5 \%$ \\
\hline Razack 2015 & 7 & 94 & 8 & 86 & $6.4 \%$ \\
\hline Shankar 2010 & 12 & 172 & 17 & 162 & $12.0 \%$ \\
\hline Thakur 2010 & 3 & 29 & 4 & 26 & $3.1 \%$ \\
\hline Ullah 2013 & 6 & 83 & 15 & 83 & $7.5 \%$ \\
\hline Wang 2013 & 32 & 768 & 20 & 392 & $20.3 \%$ \\
\hline Yerdel 2001 & 1 & 136 & 12 & 133 & $1.5 \%$ \\
\hline Subtotal $(95 \%$ CI $)$ & & 1873 & & 1470 & $73.3 \%$ \\
\hline Total events: & 81 & & 125 & & \\
\hline
\end{tabular}

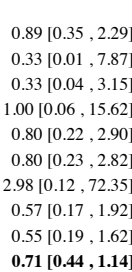

$0.71[0.44,1.14]$

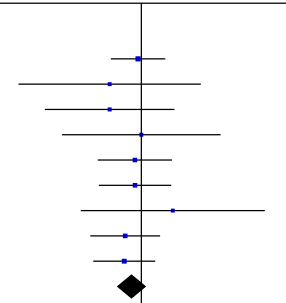

$0.60[0.15,2.44]$

$0.11[0.01,1.97]$

$0.71[0.23,2.18]$

$0.40[0.08,1.97]$

$0.15[0.04,0.66]$

$0.64[0.19,2.13]$

$0.44[0.14,1.41]$

$0.80[0.30,2.11]$

$0.66[0.33,1.35]$

$0.67[0.17,2.73]$

$0.40[0.16,0.98]$

$0.82[0.47,1.41]$

$0.08[0.01,0.62]$

$0.58[0.43,0.77]$

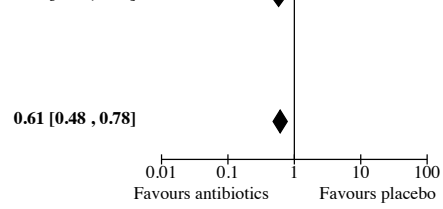

The summary RR for wound infection in the low infection risk environment subgroup was $0.71(95 \% \mathrm{Cl} 0.44$ to 1.14$)$, with no indication of heterogeneity $\left(\mathrm{Chi}^{2} \mathrm{P}=0.98\right.$ and $\left.\mathrm{I}^{2}=0 \%\right)$. For a mean background risk in the low infection risk environment control group of $2.6 \%$, the number needed to treat (NNT) to prevent one case of any type of wound infection is 133 . The summary RR for wound infections in the high risk environment was $0.58(95 \% \mathrm{Cl}$ 0.43 to 0.77 ) and again there was no indication of heterogeneity $\left(\mathrm{Chi}^{2} \mathrm{P}=0.46\right.$ and $\left.\mathrm{I}^{2}=0 \%\right)$. For a mean background risk in the high infection risk environment control group of $8.5 \%$, the NNT to prevent one case of infection is 29 . The test for subgroup differences was not significant $\left(\mathrm{Chi}^{2}=0.46\right)$, but clinical relevance justified dividing the studies into infection risk subgroups for meta-analysis.
The summary RR for all wound infections in all risk environments was $0.61(95 \% \mathrm{Cl} 0.41$ to 0.78$)$ and there were no indications of heterogeneity between studies $\left(\mathrm{Chi}^{2} \mathrm{P}=0.85\right.$ and $\left.\mathrm{I}^{2}=0 \%\right)$.

For sensitivity analysis, we excluded all studies with 'unclear' risk of bias for the domains 'selection bias', 'performance bias' or 'detection bias' and we excluded studies if we scored any of the domains as 'high risk of bias'. After exclusion, only five studies remained in the meta-analysis (Morales 2000; Aufenacker 2004; Perez 2005; Jain 2008; Wang 2013). The summary RR for all wound infections in all risk environments changed to 0.80 (95\% $\mathrm{Cl} 0.53$ to 1.21 ). In the low infection risk environment subgroup, for sensitivity analysis four studies were eligible for inclusion in the meta-analysis (Morales 2000; Aufenacker 2004; Perez 2005; Jain 
2008). This resulted in an RR of $0.78(95 \% \mathrm{Cl} 0.42$ to 1.45$)$. For the high infection risk environment subgroup, sensitivity analysis resulted in an RR of 0.82 ( $95 \% \mathrm{Cl} 0.47$ to 1.41$)$.

Funnel plot analysis showed that the low infection risk studies are symmetrically distributed (data not shown). For the high infection risk studies the asymmetrical funnel plot reveals an under-representation of studies that favour the control treatment.

\subsubsection{Superficial surgical site infections (SSSI)}

Twenty-one studies reported on superficial wound infections (SSSI) with a total of 94 superficial wound infections in the antibiotic prophylaxis group and 147 superficial wound infections in the control group (Figure 5).

\section{Figure 5. Forest plot of comparison antibiotic prophylaxis against placebo for preventing superficial wound}

infection (SSSI) after hernioplasty surgery.

\begin{tabular}{|c|c|c|c|c|c|c|c|c|c|}
\hline \multirow[b]{2}{*}{ Study or Subgroup } & \multicolumn{2}{|c|}{ Antibiotic prophylaxis } & \multicolumn{2}{|c|}{ Control } & \multirow[b]{2}{*}{ Weight } & \multirow{2}{*}{$\begin{array}{c}\text { Risk Ratio } \\
\text { M-H, Random, } 95 \% \text { CI }\end{array}$} & \multirow{2}{*}{\multicolumn{3}{|c|}{$\begin{array}{c}\text { Risk Ratio } \\
\text { M-H, Random, } 95 \% \text { CI }\end{array}$}} \\
\hline & Events & Total & Events & Total & & & & & \\
\hline \multicolumn{10}{|c|}{ 2.2.1 low infection risk environment } \\
\hline Aufenacker 2004 & 7 & 503 & 7 & 505 & $6.4 \%$ & $1.00[0.35,2.84]$ & & - & \\
\hline Bidhur 2013 & 0 & 30 & 1 & 30 & $0.7 \%$ & $0.33[0.01,7.87]$ & & & \\
\hline Goyal 2011 & 1 & 100 & 3 & 100 & $1.4 \%$ & $0.33[0.04,3.15]$ & & - & \\
\hline Jain 2008 & 1 & 60 & 1 & 60 & $0.9 \%$ & $1.00[0.06,15.62]$ & & & \\
\hline Kochhar 2014 & 4 & 106 & 5 & 106 & $4.2 \%$ & $0.80[0.22,2.90]$ & & - & \\
\hline Morales 2000 & 4 & 233 & 6 & 281 & $4.4 \%$ & $0.80[0.23,2.82]$ & & - & \\
\hline Oteiza 2004 & 1 & 124 & 0 & 123 & $0.7 \%$ & $2.98[0.12,72.35]$ & & & \\
\hline Perez 2005 & 3 & 180 & 6 & 180 & $3.7 \%$ & $0.50[0.13,1.97]$ & $\longrightarrow$ & - & \\
\hline Tzovaras 2007 & 5 & 190 & 9 & 189 & $6.0 \%$ & $0.55[0.19,1.62]$ & & - & \\
\hline Subtotal (95\% CI) & & 1526 & & 1574 & $28.4 \%$ & $0.71[0.44,1.17]$ & & & \\
\hline Total events: & 26 & & 38 & & & & & & \\
\hline \multicolumn{10}{|c|}{ Heterogeneity: $\mathrm{Tau}^{2}=0.00 ; \mathrm{Chi}^{2}=2.45, \mathrm{df}=8(\mathrm{P}=0.96) ; \mathrm{I}^{2}=0 \%$} \\
\hline \multicolumn{10}{|c|}{ Test for overall effect: $\mathrm{Z}=1.33(\mathrm{P}=0.18)$} \\
\hline \multicolumn{10}{|c|}{ 2.2.2 high infection risk environment } \\
\hline Al-Fatah 2011 & 3 & 100 & 5 & 100 & $3.5 \%$ & $0.60[0.15,2.44]$ & & - & \\
\hline Celdran 2004 & 0 & 50 & 4 & 49 & $0.8 \%$ & $0.11[0.01,1.97]$ & & - & \\
\hline Ergul 2011 & 5 & 100 & 7 & 100 & $5.6 \%$ & $0.71[0.23,2.18]$ & & - & \\
\hline Ijaz 2010 & 2 & 50 & 5 & 50 & $2.7 \%$ & $0.40[0.08,1.97]$ & & - & \\
\hline Mazaki 2013 & 2 & 100 & 13 & 100 & $3.2 \%$ & $0.15[0.04,0.66]$ & & & \\
\hline Othman 2011 & 3 & 50 & 5 & 48 & $3.7 \%$ & $0.58[0.15,2.28]$ & & - & \\
\hline Rahmani 2012 & 4 & 141 & 8 & 141 & $5.0 \%$ & $0.50[0.15,1.62]$ & & - & \\
\hline Shankar 2010 & 11 & 172 & 16 & 162 & $12.8 \%$ & $0.65[0.31,1.35]$ & & & \\
\hline Thakur 2010 & 3 & 29 & 3 & 26 & $3.0 \%$ & $0.90[0.20,4.06]$ & & & \\
\hline Ullah 2013 & 6 & 83 & 15 & 83 & $8.6 \%$ & $0.40[0.16,0.98]$ & & & \\
\hline Wang 2013 & 29 & 768 & 19 & 392 & $21.7 \%$ & $0.78[0.44,1.37]$ & & & \\
\hline Yerdel 2001 & 0 & 136 & 9 & 133 & $0.9 \%$ & $0.05[0.00,0.88]$ & & & \\
\hline Subtotal $(95 \%$ CI $)$ & & 1779 & & 1384 & $71.6 \%$ & $0.56[0.41,0.77]$ & & & \\
\hline Total events: & 68 & & 109 & & & & & & \\
\hline \multicolumn{10}{|c|}{ Heterogeneity: $\mathrm{Tau}^{2}=0.00 ; \mathrm{Chi}^{2}=10.18, \mathrm{df}=11(\mathrm{P}=0.51) ; \mathrm{I}^{2}=0 \%$} \\
\hline \multicolumn{10}{|c|}{ Test for overall effect: $Z=3.64(P=0.0003)$} \\
\hline Total $(95 \%$ CI $)$ & & 3305 & & 2958 & $100.0 \%$ & $0.60[0.46,0.78]$ & 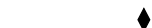 & & \\
\hline Total events: & 94 & & 147 & & & & & & \\
\hline \multicolumn{7}{|c|}{ Heterogeneity: $\mathrm{Tau}^{2}=0.00 ; \mathrm{Chi}^{2}=13.17, \mathrm{df}=20(\mathrm{P}=0.87) ; \mathrm{I}^{2}=0 \%$} & $0.005 \quad 0.1$ & 10 & 200 \\
\hline Test for overall effect: & $=3.79(\mathrm{P}=0.0$ & & & & & & Favours antibiotics & Favours $\mathrm{p}$ & lacebo \\
\hline
\end{tabular}

The average superficial wound infection incidence is $3.8 \%$, with $2.8 \%$ in the antibiotics prophylaxis group and $5.0 \%$ in the control group. The incidence of superficial wound infections in the control groups of the individual hernioplasty repair surgery studies ranged from $0 \%$ to $18.1 \%$. In the low infection risk environment studies the wound infection incidence in the control groups ranged from $0 \%$ to $4.8 \%$ and in the high infection risk environment the infection incidence in the control groups ranged from 5.0 to $18.1 \%$.
In the low infection risk environment studies the summary RR for SSSI was 0.71 (95\% Cl 0.44 to 1.17$)$, with no indication for heterogeneity $\left(\mathrm{Chi}^{2} \mathrm{P}=0.96\right.$ and $\left.\mathrm{I}^{2}=0 \%\right)$. For a mean background risk in the low infection risk environment control group of $2.4 \%$, the NNT to prevent one case of SSSI is 144. For the high infection risk environment studies the summary RR for SSSI was 0.56 (95\% $\mathrm{Cl} 0.41$ to 0.77 ). Also for this subgroup there is no indication of heterogeneity $\left(\mathrm{Chi}^{2} \mathrm{P}=0.51\right.$ and $\left.\mathrm{I}^{2}=0 \%\right)$. For a mean background 
risk in the high infection risk environment control group of $7.9 \%$, the NNT to prevent one case of SSSI is 29. Again, the test for subgroup differences was not significant $\left(\mathrm{Chi}^{2}=0.42\right)$, but also in this case clinical relevance of dividing the studies into infection risk subgroups justified subgroup analysis. The summary RR for SSSI in all risk environments was $0.60(95 \% \mathrm{Cl} 0.46$ to 0.78$)$, with no indication of heterogeneity $\left(\mathrm{Chi}^{2} \mathrm{P}=0.87\right.$ and $\left.\mathrm{I}^{2}=0 \%\right)$.

For sensitivity analysis, we excluded all studies with 'unclear' risk of bias for the domains 'selection bias', 'performance bias' or 'detection bias' and we excluded studies if any of the domains was scored 'high' risk of bias. After exclusion, only five studies remained in the meta-analysis (Morales 2000; Aufenacker 2004;
Perez 2005; Jain 2008; Wang 2013). The summary RR for superficial wound infections changed to $0.79(95 \% \mathrm{Cl} 0.51$ to 1.21$)$. In the low infection risk environment subgroup, for sensitivity analysis four studies were eligible for inclusion in the meta-analysis (Morales 2000; Aufenacker 2004; Perez 2005; Jain 2008). This resulted in an RR of 0.80 ( $95 \% \mathrm{Cl} 0.41$ to 1.56). For the high infection risk environment subgroup, sensitivity analysis resulted in an RR of 0.78 (95\% $\mathrm{Cl} 0.44$ to 1.37$)$.

\subsubsection{Deep surgical site infections (DSSI)}

Twelve studies reported on deep wound infections with a total of eight deep wound infections in the antibiotic prophylaxis group and 11 deep wound infections in the control group (Figure 6).

Figure 6. Forest plot of comparison antibiotic prophylaxis against placebo for preventing deep wound infections (DSSI) after hernioplasty surgery.

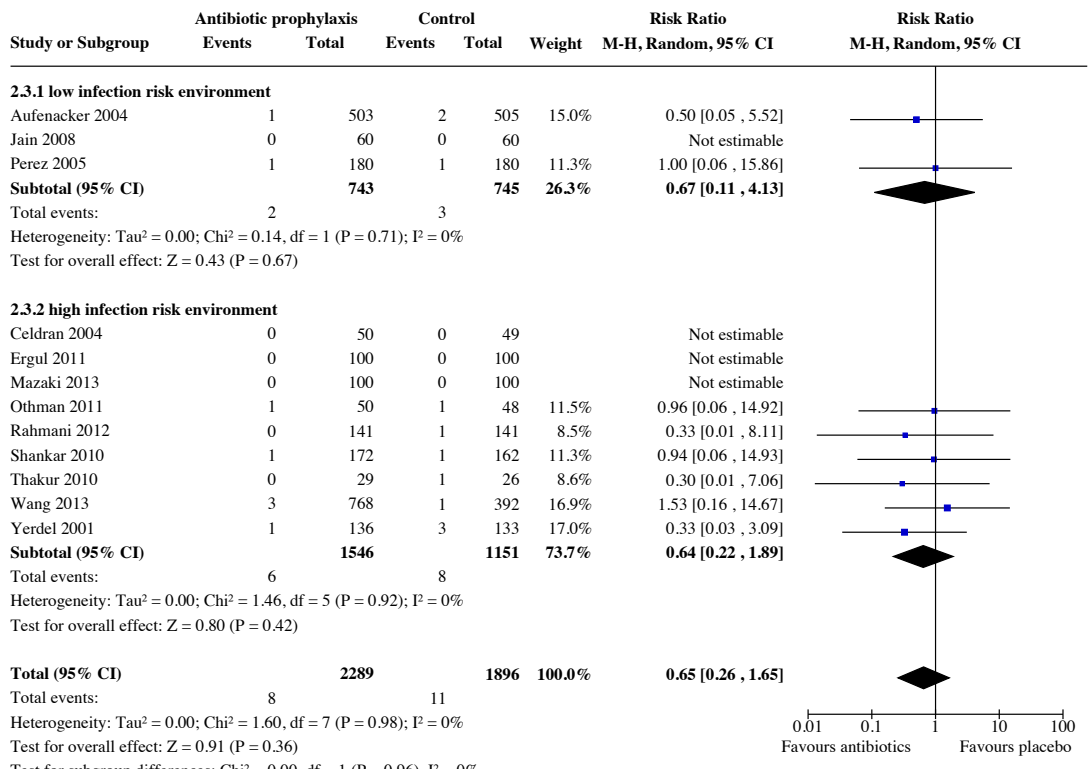

The overall deep wound infection incidence was $0.45 \%$, with $0.3 \%$ in the antibiotic prophylaxis group and $0.6 \%$ in the control group. The incidence of deep wound infections in the control groups of the individual hernioplasty repair surgery studies ranged from $0 \%$ to $3.8 \%$. In the low infection risk environment studies the wound infection incidence in the control groups ranged from $0 \%$ to $0.6 \%$ and in the high infection risk environment the infection incidence in the control groups ranged from $0 \%$ to $3.8 \%$.

In the low infection risk environment subgroup the RR for DSSI was 0.67 ( $95 \% \mathrm{Cl} 0.11$ to 4.13 ), with no indication of heterogeneity (Chi ${ }^{2}$
$\mathrm{P}=0.71$ and $\mathrm{I}^{2}=0 \%$ ). For a mean background risk in the low infection risk environment control group of $0.4 \%$, the NNT to prevent one case of DSSI is 758. In the high infection risk environment subgroup, the RR for DSSI was $0.64(95 \% \mathrm{Cl} 0.22$ to 1.89$)$, with no indication of heterogeneity $\left(\mathrm{Chi}^{2} \mathrm{P}=0.92\right.$ and $\left.\mathrm{I}^{2}=0 \%\right)$. For a mean background risk in the high infection risk environment control group of $0.7 \%$, the NNT to prevent one case of DSSI is 397. Similarly as for superficial wound infections, the test for subgroup differences was not significant $\left(\mathrm{Chi}^{2}=0.96\right)$. Also in this case, clinical relevance of dividing the studies into infection risk subgroups justified subgroup analysis. The summary RR for DSSI in all risk environments was 0.65 
(95\% Cl 0.26 to 1.65 ), with no indication for heterogeneity between studies $\left(\mathrm{Chi}^{2} \mathrm{P}=0.98\right.$ and $\left.\mathrm{I}^{2}=0 \%\right)$.

For sensitivity analysis, we excluded all studies with 'unclear' risk of bias for the domains 'selection bias', 'performance bias' or 'detection bias' and we excluded studies if any of the domains was scored 'high' risk of bias. After exclusion, four studies remained in the meta-analysis (Aufenacker 2004; Perez 2005; Jain 2008; Wang 2013). The summary RR changed to 0.93 ( $95 \% \mathrm{Cl} 0.23$ to 3.82). For the low infection risk environment subgroup, all studies could be included in the sensitivity analysis and therefore the RR did not change. For the high infection risk environment subgroup, the RR changed to 1.53 (95\% $\mathrm{Cl} 0.16$ to 14.67 ).

\section{Secondary Outcomes}

\subsection{Adverse events and development of antibiotic resistance}

The outcome 'development of antibiotic resistance' was not addressed in any of the included studies. Only one study reported that emergence of antibiotic-resistant organisms was not detected in any of the participants (Evans 1973).

Adverse events were sparsely addressed. Six studies reported that no adverse events - or more specifically that no allergic reactions - were observed (Andersen 1980; Platt 1990; Lazorthes 1992; Jain 2008; ljaz 2010; Mazaki 2013). These six studies plus a further 11 studies excluded participants allergic to antibiotics from the trial (Andersen 1980; Platt 1990; Lazorthes 1992; Taylor 1997; Yerdel 2001; Aufenacker 2004; Oteiza 2004; Perez 2005; Tzovaras 2007; Jain 2008; Ijaz 2010; Al-Fatah 2011; Ergul 2011; Bidhur 2013; Mazaki 2013; Kochhar 2014; Razack 2015). Two studies documented adverse effects: one study reported, without further explanation, that few patients $(n=6 ; 0.8 \%)$ failed to tolerate antibiotic prophylaxis (Wang 2013); the other study reported an allergic reaction (most likely due to anaesthetics) in a participant who belonged to the control group (Ergul 2011).

\section{DISCUSSION}

This review summarises the available evidence from RCTs assessing the effect of antibiotic prophylaxis on the prevention of wound infections after open inguinal or femoral hernia repair in adults. Twenty-seven trials with a total of 8308 participants were included in this review. Five trials (with 1865 participants) used a nonmesh repair technique (herniorrhaphy) and 22 trials (with 6443 participants) used a mesh repair technique (hernioplasty).

\section{Summary of main results}

For prevention of wound infections in herniorrhaphy surgery, we identified and included five studies in this review. Meta-analysis (Figure 3, Summary of findings 1) demonstrated that a beneficial effect of antibiotic prophylaxis on the prevention of wound infections could neither be confirmed nor refuted as compared to placebo (or no treatment). For a mean background risk in the control group of $4.9 \%$, the number needed to treat (NNT) to prevent one case of infection is 146 . Subgroup analysis did not alter the result of the meta-analysis and we could not perform a sensitivity analysis.

For prevention of wound infections in hernioplasty surgery, we identified and included 22 studies in this review. We present meta- analyses in Figure 4, Figure 5, Figure 6 and in the Summary of findings 2 .

The incidence of wound infections in the control groups of the individual hernioplasty repair surgery studies ranged widely from $0 \%$ to $18.1 \%$. The average superficial wound infection incidence is $4.2 \%$, with $3.2 \%$ in the antibiotics prophylaxis group and $5.5 \%$ in the control group. In the low infection risk environment studies the wound infection incidence in the control groups ranged from $0 \%$ to $4.8 \%$ and in the high infection risk environment the infection incidence in the control groups ranged from $5.0 \%$ to $18.1 \%$. The average deep wound infection (DSSI) rate in the low infection risk environment was $0.34 \%$ and in the high infection risk environment was $0.52 \%$. This is consistent with reported rates in the literature (Mann 1998; Robbins 1998; Stephenson 2003; Fawole 2006).

In the low infection risk environment a beneficial effect of antibiotic prophylaxis on the prevention of all wound infections (SSSI + DSSI) as well as on superficial wound infections could neither be confirmed nor refuted. The anticipated absolute effect in a lowrisk environment shows that the incidence of 26 wound infections (SSSI + DSSI) per 1000 participants in the placebo (or no treatment) group is lowered to 18 wound infections (SSSI + DSSI) per 1000 participants in the antibiotic prophylaxis group (Summary of findings 2). Thus, for a mean background risk in the low infection risk environment control group of $2.6 \%$, the NNT to prevent one case of any type of wound infection is 133 . Similar results are observed for superficial wound infections (Summary of findings 2). For a mean background risk in the low infection risk environment control group of $2.4 \%$, the NNT to prevent one case of SSSI is 144 .

In contrast, a beneficial effect of antibiotic prophylaxis on prevention of all types of wound infection (SSSI + DSSI) as well as of superficial wound infections was demonstrated in the high infection risk environment. This result reflects the importance of taking aseptic operation (room) conditions and wound infection background risk into account when making a clinical decision on antibiotic prophylaxis administration during open hernioplasty surgery. The anticipated absolute effect in a low-risk environment shows that the incidence of 85 wound infections (SSSI + DSSI) per 1000 participants in the placebo (or no treatment) group is lowered to 49 wound infections (SSSI + DSSI) per 1000 participants in the antibiotic prophylaxis group (Summary of findings 2). Thus, for a mean background risk in the high infection risk environment control group of $8.5 \%$, the NNT to prevent one case of any type of wound infection is 29 . Similar results were observed for superficial wound infections (Summary of findings 2). For a mean background risk in the high infection risk environment control group of $7.9 \%$, the NNT to prevent one case of SSSI is 29. Upon combination of the two subgroups, a beneficial effect of antibiotic prophylaxis as compared to placebo (or no treatment) was still detected. However, upon exclusion of the trials at high risk of bias, few trials could be included into the meta-analysis and the beneficial effect of antibiotic prophylaxis could no longer be established.

The prevention of deep wound infections (DSSI) by antibiotic prophylaxis could neither be confirmed nor refuted as compared to placebo (or no treatment). Subgroup analysis or sensitivity analysis did not change these results. The anticipated absolute effect in the low infection risk environment shows that the incidence of four deep wound infections per 1000 participants in the placebo (or no treatment) group is lowered to three deep wound infections per 1000 participants in the antibiotic prophylaxis group (Summary of 
findings 2). Hence, for a mean background risk in the low infection risk environment control group of $0.4 \%$, the NNT to prevent one case of DSSI is 758. The anticipated absolute effect in the high infection risk environment shows that the incidence of seven deep wound infections per 1000 participants in the placebo (or no treatment) group is lowered to four deep wound infections per 1000 participants in the antibiotic prophylaxis group (Summary of findings 2). Hence, for a mean background risk in the high infection risk environment control group of $0.7 \%$, the NNT to prevent one case of DSSI is 397

\section{Overall completeness and applicability of evidence}

For the effect of antibiotic prophylaxis on the prevention of wound infections after open inguinal or femoral hernia repair in adults, 27 placebo-controlled studies with 8308 participants are currently available. These include studies published over a period of more than 40 years. Hernia repair method, operation room facilities and applied antibiotics prophylaxis regimes etc. have changed over time. This results in clinical heterogeneity. To address this issue, we have distinguished in our meta-analysis between two hernia repair methods (i.e. herniorrhaphy and hernioplasty). By doing so, the herniorrhaphy studies with an older publication date were separated from the more recently published hernioplasty studies, thereby reducing clinical heterogeneity between studies. Given the strong improvement of the operation room facilities over the years, it is questionable whether the herniorrhaphy study results can be extrapolated to the current situation. On the other hand, herniorrhaphy surgery is nowadays not performed to a great extent and therefore presented results in this review may not be relevant to current clinical practice.

\section{Study design and treatment characteristics}

The number of participants in seven studies were small $(\leq 100$ participants) (Evans 1973; Celdran 2004; ljaz 2010; Thakur 2010; AlFatah 2011; Othman 2011; Bidhur 2013), which made interpretation of the results of these single studies difficult. The herniorrhaphy studies were few and total number of participants in the metaanalysis did not approach optimal information size. In contrast, we included a large number of hernioplasty studies (and participants) into meta-analyses in this review and optimal information was nearly reached for the outcomes 'all wound infections' and 'superficial wound infections'.

The subject of antibiotic prophylaxis is a complicated issue; only doses that exceed the therapeutic level are effective. This is dependent on many factors including the (number of) dose(s), route and timing of administration, type of antibiotic, and presence of antibiotic-resistant bacterial species in the geographical area of the study. The included studies varied in the type of administered antibiotics, the frequency and timing of administration (see also Characteristics of included studies; Table 1; Table 2). Despite the variation in antibiotic prophylaxis regimes, heterogeneity analysis revealed low levels of heterogeneity between studies. Moreover, large overlap of the $95 \% \mathrm{Cls}$ of the various RRs was observed. Altogether, this indicates the absence of important differences between antibiotic regimens. This is supported by the results of a recent systematic review investigating the effects of different antibiotic types on wound infection prevention. Boonchan 2017 analysed betalactam inhibitors and first-generation cephalosporins and other antibiotics for wound infection prevention. They demonstrated a non-significant difference between antibiotics classes in wound infection prevention effectiveness. However, it is unclear whether herniorrhaphy and hernioplasty require the same antibiotic approach, given the many differences between the surgical methods.

Aseptic operation (room) conditions, length of the surgical procedure, drain usage and skin preparation (including shaving) are considered risk factors for wound infection development (Mangram 1999); these factors could have contributed to the large differences in baseline wound infection percentages we observed between studies. Hernia surgery is considered a clean surgical procedure with wound infection percentages in lowrisk patients and short surgical procedures $(<1$ hour) of below 2\% (Mangram 1999). Baseline wound infection percentages as high as $18.1 \%$ that we observed do not fall within the expected wound infection percentage range of $0 \%$ to $2 \%$. For this reason, we performed subgroup analysis for low and high infection risk environments. These analyses revealed that antibiotic prophylaxis has different preventive effects in different environments, geographical locations and clinical settings/policies.

\section{Participants characteristics and outcome assessment}

Overall, participant characteristics and wound infection risk factors (i.e. age, BMI or ASA-status) were not consistently or specifically reported in the included studies (Mangram 1999). When they were reported, participants vary widely in risk factors. This makes data interpretation difficult. Nevertheless, most studies excluded patients with co-morbidities (such as diabetes mellitus, malignancy, HIV) and high ASA status and therefore the results of this review seem to be applicable to low-risk patients. It is currently unclear which patient characteristics are significant to distinguish a low-risk from a high-risk patient.

Previous versions of the review made no distinction between superficial and deep wound infections. This distinction is relevant to both the patient and clinical practice. Here, we did analyse superficial and deep wound infections separately and therefore results are more applicable to clinical practice. It should be noted that several studies made no clear distinction between SSSI and DSSI. These studies were excluded from meta-analysis for the separate outcomes.

Accurate assessment of wound infections is challenging, as there is a subjective component in the diagnosis of a wound infection. New tools and higher quality standards are continually being developed to more accurately assess surgical site infection (Bluebelle Study Group 2018; GlobalSurg Collaborative 2018). Studies included in this review applied many different wound infection assessment tools and definitions which are not consistent with the latest highquality standards. Additionally, dichotomisation of any outcome inherently results in a loss of information. The outcome 'presence of wound infection' versus 'absence of wound infection' (SSSI or DSSI) does not contain information on the severity of the infection. This makes the comparison between included studies with different outcome assessment tools difficult. Nevertheless, treatment and control groups within studies can be compared as they were assessed in a similar manner. Altogether, the data should be interpreted with caution as outcome assessment did not meet the high current standards and outcomes are assessed with different measures. 
The follow-up periods varied between studies, but most studies reported various short follow-up periods of several days to one month postoperatively. Superficial wound infection occurs by definition within one month postoperatively (Mangram 1999). For this reason, we pooled all these studies in the meta-analyses and are confident that no or an insignificantly small number of infections were missed. In contrast, deep wound infections can very occasionally occur up to years postoperatively, especially in low infection risk environments when multifilament meshes are implanted. Most hernioplasty studies had shorter follow-up periods than one year. We are not, therefore, completely confident that all late-onset deep wound infections were detected. Due to the low incidence of late-onset deep wound infections, very large trials are required to provide a sufficient number of events to address this question, especially when taking into account that deep wound infections usually require either surgical drainage or mesh removal (in case of multifilament meshes). Therefore deep wound infections are considered as a serious complication (Mann 1998; Fawole 2006).

\section{Quality of the evidence}

The overall methodological quality ranged from very low to moderate. Quality of the evidence was very low for the herniorrhaphy studies for the outcome 'all wound infections' (see Summary of findings 1). Quality of the evidence in hernioplasty surgery was very low to moderate for the outcome 'all wound infections' and the outcome 'superficial wound infections' (see Summary of findings 2). Quality of the evidence for the outcome 'deep wound infections' in hernioplasty surgery was low to moderate (see Summary of findings 2).

Wound infection is a binomial outcome measurement with a low incidence. To obtain an optimal information size (number of infection events) for meta-analysis of the pooled results, a large participant population (> 2000 participants per treatment arm) is required. For several meta-analyses performed in this review this optimal information size was not reached and therefore quality of the evidence was downgraded for imprecision (herniorrhaphy studies, hernioplasty studies for all outcomes in subgroup analyses).

Besides downgrading for imprecision, we also downgraded for risk of bias. A large number of studies did not describe the randomisation or allocation concealment procedures in great detail, which resulted in an unclear risk of selection bias. There is a subjective component in the clinical judgement of wound infections, despite the fact that wound infections are defined in great detail by CDC guidelines. Blinding of outcome assessment is therefore important. Several studies (all herniorrhaphy and seven hernioplasty) did not describe blinding of outcome assessment and were scored 'unclear risk of detection bias'. One herniorrhaphy study was scored 'high risk of detection bias', as surgeons who were not blinded for the treatment also performed outcome assessment.

Five studies scored 'high risk of bias' for attrition bias (two herniorrhaphy and three hernioplasty studies) as the number of missing data (participants lost to follow-up or participants excluded from the analysis) was high. The proportion of missing outcomes compared with the observed event risk was large enough to have induced clinically relevant bias in the intervention effect estimate.
One study scored 'high risk of selective reporting bias' as the study protocol was registered after the trial was terminated. It should be noted that many studies did not register their study protocol in a prospective trial register. Since most of these studies did report on all expected outcomes, however, we scored these studies as 'low risk of bias' for selective reporting.

Finally, three hernioplasty studies scored 'high risk of other bias' as these studies were terminated after interim analysis of the results. This may have resulted in an increased benefit for the antibiotics prophylaxis group. Overall, studies within the subgroup 'low infection risk environment' had scored more items at low risk of bias than the studies in the subgroup 'high infections risk environment'.

\section{Potential biases in the review process}

We searched several electronic databases and prospective trial registers, performed citation searching and we contacted experts in the field to obtain all relevant studies for this review. Nevertheless, it is always possible that we missed relevant studies or data. Two authors independently performed data extraction and quality assessment of the studies to minimise bias in the review process. For several studies we could not obtain all the data required to make judgements for all risk of bias from the publication. In those cases we tried to contact study authors to retrieve additional information. Unfortunately, very few authors responded to our request and the information provided did not affect our initial risk of bias judgement.

For some meta-analyses (i.e. herniorrhaphy studies and DSSI outcome) we did not perform a publication bias (funnel plot) analysis, because too few studies ( $<10$ studies) were available for these analyses.

Reporting of adverse effects was scarce. Seventeen studies excluded participants with an allergy to antibiotics; this particular adverse event was therefore not detected in those studies. The outcome 'development of antibiotic-resistant pathogens' was only addressed in one study. The short administration period of antibiotic prophylaxis during surgery is not likely to result in the development of antibiotic-resistant bacterial strains. It would, however, be of interest if study authors had reported the antibiotic resistance profile of the bacterial strains that were present in wound infections. This information gives an estimate of the incidence of antibiotic-resistant strains causing postoperative wound infections. Also it could provide an explanation for the ineffectiveness of the applied antibiotic prophylaxis in the patients with wound infections.

\section{Agreements and disagreements with other studies or reviews}

A number of (systematic) reviews and clinical guidelines reported on antibiotic prophylaxis intervention compared to placebo (or no treatment) treatment for prevention of wound infections in open inguinal or femoral hernioplasty surgery. Depending on the publication date of review a smaller or more comprehensive amount of studies was included in the meta-analysis. Moreover, most reviews did not perform a meta-analysis of separate high and low infection risk subgroups. No systematic reviews with a metaanalysis of herniorrhaphy surgery were found. 
Biswas 2005 included four RCTs and a few retrospective studies and summarised the data narratively. They concluded that antibiotic prophylaxis is required for hernioplasty repair. Reviews of later publication date revealed a protective effect of antibiotic prophylaxis, similar to the results reported in this review. Sanabria 2007 (meta-analysis of six studies), Yin 2012 (meta-analysis of nine studies) and Li 2012 (meta-analysis of six studies) revealed a protective effect of antibiotic prophylaxis on postoperative wound infection incidence after hernioplasty surgery. These reviews included studies of low and high infection risk environment and expressed the pooled effect size as odds ratio (OR). They did not analyse high and low infection risk studies separately.

Similar to the results presented in this review, some systematic reviews analysed superficial and deep wound infections in separate meta-analyses. In their analysis, Aufenacker 2006 (meta-analysis of six studies) was unable to show a significant reduction of the OR for wound infections in the antibiotic prophylaxis treatment group. The systematic reviews of Mazaki 2013 and Erdas 2016 included a larger number of studies (respectively 12 and 16 studies) and were able to reveal a protective effect, expressed as pooled OR, of antibiotic prophylaxis on superficial wound infections, but not on deep wound infections.

International guidelines included 12 and 16 RCTs in their metaanalysis (Miserez 2014; HerniaSurge Group 2018). Separate metaanalyses for a low and high infection risk environment were performed. The definition of low infection risk $<5 \%$ infection risk) and high infection risk (> $5 \%$ infection risk) is similar to the definition used in this review. Both guidelines reveal a beneficial effect, expressed as OR, of antibiotic prophylaxis on wound infection prevention in a high infection risk environment, but not in a low infection risk environment. Miserez 2014 presented a separate analysis for deep wound infections. In their analysis no significant benefit of antibiotic prophylaxis on wound infection prevention was observed.

Some reviews applied patient inclusion criteria more strictly. Our meta-analysis included eight studies that also included a small number of adolescent participants (Oteiza 2004; Tzovaras 2007; Shankar 2010; Al-Fatah 2011; Goyal 2011; Wang 2013; Kochhar 2014; Razack 2015). Excluding these studies from our meta-analysis did not affect the summary RR to a great extent (RR for all wound infections in all risk environments changed to $0.5(95 \% \mathrm{Cl} 0.35$ to 0.72 ). Therefore we believe that including a small number of participants aged younger than 18 years has not affected our results.

Altogether, the results presented in these (systematic) reviews are in line with the results presented here. The reviews described above and previous versions of this review used the OR as effect estimate. In the current version of this review we have presented the results as RR, which is a more appropriate effect measure for presenting the results of prospective studies (Altman 1991). For rare outcome events, however, such as postoperative wound infections in inguinal or femoral hernia surgery, the odds ratio (OR) and risk ratio ((RR) is approximately the same (Altman 1991). Therefore the results between the previous and current version of this review have only slightly changed, despite the fact that more studies were included in the meta-analysis. In the previous version of this review the OR (fixed-effect model) for all wound infections was 0.56 (95\% $\mathrm{Cl} 0.38$ to 0.81 ). The RR (random-effects model) presented here for all wound infections in all risk environments is $0.61(95 \% \mathrm{Cl}$
0.48 to 0.78 ). For comparison we have also computed the $\mathrm{OR}$. Here, the OR for all wound infections in all risk environments in hernioplasty surgery studies is $0.59(95 \% \mathrm{Cl} 0.46$ to 0.77$)$. In the current version of this review, more outcomes (all wound infections, superficial and deep wound infections) and subgroups (low and high infection risk environment) are reported than in the previous versions. Altogether, this review presents a more comprehensive analysis of more outcomes and subgroups than the above-mentioned systematic reviews and guidelines.

\section{AUTHORS' CONCLUSIONS}

\section{Implications for practice}

Administration of antibiotic prophylaxis for elective open inguinal or femoral hernia repair cannot be universally recommended. For open herniorrhaphy surgery we are uncertain whether antibiotic prophylaxis prevents the development of all types of postoperative wound infections. For open hernioplasty surgery, it is uncertain whether antibiotic prophylaxis reduces all types and superficial wound infections in a high infection risk environment. In a low infection risk environment antibiotic prophylaxis makes little or no difference in reducing these type of wound infections.Regarding deep wound infections, antibiotic prophylaxis probably makes little or no difference in preventing the development of these type of wound infections after open hernioplasty surgery.

\section{Implications for research}

This review and meta-analysis included a large number of studies for the outcome 'superficial wound infections'. Unfortunately, the follow-up period of these studies was too limited to address the development of the rare phenomenon of late-onset deep wound infections. Currently, the number of events for the outcome 'deep infections' is too small to draw firm conclusions. It could be beneficial for future studies to include high numbers of participants ( $>1000$ participants per study arm) and to extend follow-up periods to two years postoperatively in order to detect sufficient numbers of deep wound infections, which have an incidence lower than $1 \%$. Currently monofilament meshes are usually implanted, however, and these meshes do not require removal upon deep wound infection. The clinical relevance of such studies might therefore be limited. In their review Sanabria and colleagues calculated that larger trials (1600 to 3000 participants) are required to obtain enough wound infection events to make a $50 \%$ decrease in antibiotic prophylaxis versus placebo (depending on the background rate of wound infections) (Sanabria 2007). To more adequately address the outcome of 'wound infections', it would be highly beneficial if future studies would apply higher quality standards for outcome assessment. Standards such as Bluebelle Wound healing Questionnaire or the Clavien-Dindo classification system provide more insight into severity of postoperative wound complications and healing (Dindo 2004; Bluebelle Study Group 2018).

To be able to gain more insight into the patient population for which the results reported here are most applicable, future studies should report in more detail relevant baseline characteristics of risk factors of participants and operation characteristics (ASA-status, BMI, age, co-morbidities, operative time length and skin preparation etc.). Identification of risk factors for postoperative wound infection after open inguinal or femoral hernioplasty surgery would be useful to 
identify those groups of patients that may benefit from antibiotic prophylaxis.

It would be of interest if study authors for future studies would report on the antibiotic resistance profile of the bacterial strains that were present in wound infections. This information gives an estimate of the incidence of antibiotic-resistant strains causing postoperative wound infections. Also it provides a possible explanation for the ineffectiveness of the administered antibiotic prophylaxis. Finally, a cost-effectiveness analysis to evaluate the advantages of antibiotic prophylaxis versus the cost of prolonged hospital stay due to wound infection is needed to appropriately appraise the economic implications. The cost of development of antibiotic resistant bacteria should also be taken into account in such analyses.

\section{ACKNOWLEDGEMENTS}

Very special thanks to Javier Lozano-Garcia and Juan SecoGil for their invaluable contribution; and special thanks to the
Iberoamerican Cochrane Centre, particularly to Marta Roqué, for providing statistical advice to the previous versions of this review. We would like to thank Henning Andersen, Jason Rosenberg and Kristoffer Andresen from the Cochrane Colorectal Review Group for assistance.

Special thanks to the Dutch Cochrane Center for providing advice and assistance for this review update, especially to Rene Spijker, information specialist, for performing the literature search in MEDLINE, Embase and Cochrane Registry of Studies Online; and to Pauline Heus and Lotty Hooft for helpful discussions. Also, we would like to thank Bita Mesgarpour, Cochrane Iran, for providing assistance in data extraction and 'Risk of bias' analysis of Rahmani 2012, which is written in Farsi. We thank Steven Thijsen, Diakonessenhuis Utrecht, for critically reviewing the antimicrobial background information. 


\section{REFEREN CES}

\section{References to studies included in this review}

Al-Fatah 2011 \{published data only\}

Al-Fatah, MA. The role of antibiotic prophylaxis in prevention of wound infection after Lichtenstein repair of primary inguinal hernia. Al-Azhar Assiut Medical journal 2011;9(1):173-80.

\section{Andersen 1980 \{published data only\}}

* Andersen JR, Burcharth F, Larsen HW, Roder O, Andersen B. Polyglycolic acid, silk, and topical ampicillin. Their use in hernia repair and cholecystectomy. Arch Surg 1980;115:293-5.

\section{Aufenacker 2004 \{published data only\}}

Aufenacker TJ, van Geldere D, van Mesdag T, Bossers AN, Dekker B, Scheije E et al. The role of antibiotic prophylaxis in prevention of wound infection after Lichtenstein open mesh repair of primary inguinal hernia. A multicenter double-blind randomized controlled trial. Annals of Surgery 2004;240(6):955-61.

\section{Bidhur 2013 \{published data only\}}

Bidhur KC, Regmi R, Agrawal CS, Pathania, OP. Role of single dose antibiotic prophylaxis in prevention of wound infection following lichtenstein inguinal hernioplasty; a randomized clinical trial. Nepal Journal of Medical Sciences December 2013;2(2):108-13.

\section{Celdran 2004 \{published data only\}}

Celdran A, Frieyro O, de la Pinta JC, Souto JL, Esteban J, Rubio JM et al. The role of antibiotic prophylaxis on wound infection after mesh hernia repair under local anaesthesia on an ambulatory basis. Hernia 2004;8(1):20-2.

\section{Ergul 2011 \{published data only\}}

Ergul Z, Akinci M, Urgulu C, Kulacoglu H, Yilmaz KB. Prophylactic antibiotic use in elective inguinal hernioplasty in a trauma center. Hernia 2012;16(2):145-51.

Ergul Z. Antibiotic Prophylaxis in Mesh Repair of Inguinal Hernias. Clinicaltrials.gov 2010. [NCT01264003]

Kulacoglu $\mathrm{H}$. The possible benefit of prophylactic antibiotic use in patients undergoing unilateral elective inguinal hernia repair with prosthetic material.. ISRCTNregistry 2010. [ISRCTN85660082]

\section{Evans 1973 \{published data only\}}

Evans C, Pollock AV. The reduction of surgical wound infections by prophylactic parenteral cephaloridine. A controlled clinical trial. British Journal of Surgery 1973;60(6):434-7.

Goyal 2011 \{published data only\}

Goyal A, Garg R, Jenaw RK, Jindal DK. Role of Prophylactic Antibiotics in Open Inguinal Hernia Repair: A Randomised Study. Indian J Surg 2011;73(3):190-193.

ljaz 2010 \{published data only\}

ljaz A, Amer SM. Postopereative wound infection; Prevention "The role of antibiotic prophylaxis in Lichtenstein". Professional Medical Journal 2010;17(2):174-9.

\section{Jain 2008 \{published data only\}}

Jain SK, Jayant M, Norbu C. The role of antibiotic prophylaxis in mesh repair of primary inguinal hernias using prolene hernia system: a randomized prospective double-blind control trial. Tropical Doctor 2008;38(2):80-2.

\section{Kochhar 2014 \{published data only\}}

Kochhar G, Andley M, Saha S, Kumar A, Pusuluri R, Saurabh G, A Kumar. Randomized controlled trial to evaluate the role of antibiotic prophylaxis in lichtenstein hernia repair. Journal of Young Medical Researchers 2014;1(2):1-8.

\section{Lazorthes 1992 \{published data only\}}

Lazorthes F, Chiotasso P, Massip P, Materre JP, Sarkissian M. Local antibiotic prophylaxis in inguinal hernia repair. Surgery, Gynecology \& Obstetrics 1992;175(6):569-70.

\section{Mazaki 2013 \{published data only\}}

Mazaki T, Mado K, Masuda H, Shiono M, Tochikura N, Kaburagi M. A randomized trial of antibiotic prophylaxis for the prevention of surgical site infection after open mesh-plug hernia repair. American Journal of Surgery 2013;207(4):476-84.

Mazaki T. Prophylactic Antibiotic Use in Hernioplasty (RCTherniation). ClinicalTrials.gov 2008. [NCT00636831]

\section{Morales 2000 \{published data only\}}

Morales R, Carmona A, Pagán A, García Menéndez C, Bravo R, Hernández MJ et al. Utility of antibiotic prophylaxis in reducing wound infection in inguinal or femoral hernia repair using polypropylene mesh [Utilidad de la profilaxis antibiótica en la reducción de la infección de herida en la reparación de la hernia inguinal o crural mediante malla de polipropileno]. Cirugia Espanola 2000;67(1):51-9.

\section{Oteiza 2004 \{published data only\}}

Oteiza F, Ciga MA, Ortiz H. Antibiotic prophylaxis in inguinal hernioplasty [Profilaxis antibiótica en la hernioplastia inguinal]. Cirugia Espanola 2004;75(2):69-71.

\section{Othman 2011 \{published data only\}}

Othman I. Prospective randomized evaluation of prophylactic antibiotic usage in patients undergoing tension free inguinal hernioplasty. Hernia 2011;15(3):309-13.

\section{Perez 2005 \{published data only\}}

Perez AR, Roxas MF, Hilvano SS. A randomized, double-blind, placebo-controlled trial to determine effectiveness of antibiotic prophylaxis for tension-free mesh herniorrhaphy. Journal of the American College of Surgeons 2005;200(3):393-7.

\section{Platt 1990 \{published data only\}}

Platt R, Zaleznik DF, Hopkins CC, Dellinger EP, Karchmer AW, Bryan CS et al. Perioperative antibiotic prophylaxis for herniorrhaphy and breast surgery. New England Journal of Medicine 1990;322(3):153-60. 


\section{Rahmani 2012 \{published data only\}}

Kermansaravi M, Rahmani N. Evaluation of prophylactic antibiotic effectiveness in prevention of wound infection in lichtenstein hernioplasty with mesh.. IRCT 2012. [IRCT201203121786N4]

Rahmani N, Saravi MK, Sayadi S, Khalilian A. Effect of prophylactic antibiotics in preventing wound infection in hernioplasty with inguinal mesh in Lichtenstein method. Journal of Mazandaran University of Medical Sciences 2012;22(90):88-93.

\section{Razack 2015 \{published data only\}}

Razack A, Kapoor KK, Tambat RM. The role of single dose antibiotic prophylaxis in open mesh repair of inguinal hernia:a prospective, double blind randomized trial. Journal of Evolution of Medical and Dental Sciences 2015;4(35):6017-26.

\section{Shankar 2010 \{published data only\}}

Shankar VG, Srinivasan K, Sistla SC, Jagdish S. Prophylactic antibiotics in open mesh repair of inguinal hernia. A randomized controlled trial. International Journal of Surgery (London, England) 2010;8(6):444-7.

\section{Taylor 1997 \{published data only\}}

Taylor EW, Byrne DJ, Leaper DJ, Karran SJ, Browne MK Mitchell KJ. Antibiotic prophylaxis and open groin hernia repair. World Journal of Surgery 1997;21(8):811-5.

Thakur 2010 \{published data only\}

Thakur L, Upadhyay S, Peters NJ, Saini N, Deodhar M. Prophylactic antibiotic usage in patients undergoing inguinal mesh hernioplasty - A clinical study. Indian Journal of Surgery 2010;72(3):240-2.

\section{Tzovaras 2007 \{published data only\}}

Tzovaras G, Delikoukos S, Christodoulides G, Spyridakis M, Mantzos F, Tepetes K, et al. The role of antibiotic prophylaxis in elective tension-free mesh inguinal hernia repair: results of a single-centre prospective randomised trial. International Journal of Clinical Practice 2007;61(2):236-9.

Ullah 2013 \{published data only\}

Ullah B, Khan SA, Ahmed S, Pasha T. Efficacy of preoperative single dose antibiotic in patients undergoing mesh repair for inguinal hernia. Journal of Ayub Medical College, Abbottabad 2013;25(1-2):103-5.

\section{Wang 2013 \{published data only\}}

Wang J, Ji G, Yang Z, Xi M, Wu Y, Zhao P, Wang L, Yu W, Wen A. Prospective randomized, double-blind, placebo controlled trial to evaluate infection prevention in adult patients after tensionfree inguinal hernia repair. International Journal of Clinical Pharmacology and Therapeutics 2013;51(12):924-31.

Wen A. Factors Related to Infection Prevention After Tensionfree Inguinal Hernia Repair (infection). Clinicaltrials.gov 2013. [NCT01802606]

\section{Yerdel 2001 \{published data only\}}

Yerdel MA, Akin EB, Dolalan S, Turkcapar AG, Pehlivan M, Gecim IE, et al. Effect of single-dose prophylactic ampicillin and sulbactam on wound infection after tension-free inguinal hernia repair with polypropylene mesh: the randomized, double-blind, prospective trial. Annals of Surgery 2001;233(1):26-33.

\section{References to studies excluded from this review}

Ahmed 2014 \{published data only\}

Ahmed QJ, Suchdev SD, Anwar MA, Hasan A, Sarfaraz. To detect the use of prophylactic antibiotics in inguinal hernia repair: $A$ randomized study in tertiary care hospital Karachi. Pakistan Journal of Medical and Health Sciences 2014;8(4):976-978.

\section{Aufenacker 2006 \{published data only\}}

Aufenacker, TJ, Koelemay, MJ, Gouma, DJ, Simons, MP. Systematic review and meta-analysis of the effectiveness of antibiotic prophylaxis in prevention of wound infection after mesh repair of abdominal wall hernia. British Journal of Surgery 2006;93(1):5-10.

\section{Barreca 2000 \{published data only\}}

Barreca M, Stipa F, Cardi E, Bianchini L, Lucandri G, Randone B. Antibiotic prophylaxis in the surgical treatment of inguinal hernia: need or habit? Minerva Chirurgica 2000;55(9):599-605.

\section{Bhuiyan 2017 \{published data only\}}

Bhuiyan MNA. Efficacy of single dose antibiotic prophylaxis in open tension free mesh inguinal hernioplasty. In: British Journal of Surgery: Association of Surgeons of Great Britain \& Ireland (ASBGI) 2017. Vol. 104. 2017:185.

\section{Boonchan 2017 \{published data only\}}

Boonchan T, Wilasrusmee C, McEvoy M, Attia J, Thakkinstian A. Network meta-analysis of antibiotic prophylaxis for prevention of surgical-site infection after groin hernia surgery. British Journal of Surgery 2017;104(2):e106-e117.

\section{Erdas 2016 \{published data only\}}

Erdas E, Medas F, Pisano G, Nicolosi A, Calo PG. Antibiotic prophylaxis for open mesh repair of groin hernia: systematic review and meta-analysis. Hernia 2016;20(6):765-76.

\section{Esposito 2006 \{published data only\}}

Esposito S, Leone S, Noviello S, Ianniello S, Marvaso F, Cuniato $A$, et al. Antibiotic prophylaxis in hernia repair and breast surgery: A prospective randomized study comparing piperacillin/tazobactam versus placebo. Journal of Chemotherapy (Florence, Italy) 2006;18(3):278-284.

\section{Gierhake 1975 \{published data only\}}

Gierhake FW. [Antibiotics and their indications in surgery]. Der Chirurg; Zeitschrift fur Alle Gebiete der Operativen Medizen 1975;46(1):10-5.

\section{Lazorthes 1993 \{published data only\}}

Lazorthes F, Chiotasso P, Massip P, Materre JP, Sarkissian M. Prophylaxis of infection at site of inguinal hernia repair of pre- 
operative local antibiotic administration [French]. Journal de Chirurgie 1993;130(12):507-9.

Leon 2011 \{published data only\}

Leon JS, Acevedo AF, Riosecor DR, Vasquez JV, Dellepiane VEU. Antibiotic prophylaxis in mesh inguinal herniorrhapy. Revista Chilena De Cirugia 2011;63(2):186-90. [DOI: http:// dx.doi.org/10.4067/s0718-40262011000200009]

\section{Li 2012 \{published data only\}}

Li J-F, Lai D-D, Zhang X-D, Zhang A-M, Sun K-X, Luo H-G, et al. Meta-analysis of the effectiveness of prophylactic antibiotics in the prevention of postoperative complications after tensionfree hernioplasty. Canadian Journal of Surgery 2012;55(1):27-32.

Mazaki 2013b \{published data only\}

Mazaki T, Mado K, Masuda H, Shiono M. Antibiotic prophylaxis for the prevention of surgical site infection after tension-free hernia repair: a Bayesian and frequentist meta-analysis. Journal of the American College of Surgeons 2013;217(5):788-801.

\section{Mehrabi Bahar 2015 \{published data only\}}

Mehrabi Bahar M, Jabbari Nooghabi A, Jabbari Nooghabi M, Jangjoo A. The role of prophylactic cefazolin in the prevention of infection after various types of abdominal wall hernia repair with mesh. Asian Journal of Surgery 2015;38(3):139-44.

Pessaux 2006 \{published data only\}

Pessaux P, Lermitte E, Blezel E, Msika S, Hay J-M, Flamant Y, et al. Predictive risk score for infection after inguinal hernia repair. American Journal of Surgery 2006;192(2):165-71.

\section{Sanabria 2007 \{published data only\}}

Sanabria A, Dominguez LC, Valdivieso E, Gomez G. Prophylactic antibiotics for mesh inguinal hernioplasty: a meta-analysis. Annals of Surgery 2007;245(3):392-6.

\section{Sanchez-Manuel 2003 \{published data only\}}

Sanchez-Manuel FJ, Seco-Gil JL. Antibiotic prophylaxis for hernia repair. Cochrane Database of Systematic Reviews 2003, Issue 4. Art. No: CD003769. [DOI: 10.1002/14651858.CD003769]

\section{Sanchez-Manuel 2012 \{published data only\}}

Sanchez-Manuel JF, Lozano-Garcia J, Seco-Gil JL. Antibiotic prophylaxis for hernia repair. Cochrane Database of Systematic Reviews 2012, Issue 2. Art. No: CD003769. [DOI: 10.1002/14651858.CD003769.pub4]

Taylor 1996 \{published data only\}

Taylor EW, Byrne D, Karran S, Leaper D, Browne MK, Mitchell KG. Is antibiotic prophylaxis indicated for open groin hernia repair? British Journal of Surgery 1996;83(S2):61.

\section{Yin 2012 \{published data only\}}

Yin Y, Song T, Liao B, Luo Q, Zhou Z. Antibiotic prophylaxis in patients undergoing open mesh repair of inguinal hernia: a meta-analysis. American Surgeon 2012;78(3):359-65.

\section{References to studies awaiting assessment}

Barkhordary 2012 \{unpublished data only\}

\section{Additional references}

\section{Altman 1991}

Altman DG. Practical Statistics for medical Research. Chapman \& Hall, 1991.

\section{Bailey 1992}

Bailey IS, Karran SE, Toyn K, Brough P, Ranaboldo C, Karran SJ. Community surveillance of complications after hernia surgery. BMJ 1992;304(6825):469-71.

\section{Bassini 1890}

Bassini E. [About the treatment of inguinal hernia] [Ueber die Behandlung des Leistenbruches]. Langenbecks Arch Klin Chir 1890;40:429-76.

\section{Berard 1964}

Berard F, Gandon J (National Academy of Sciences, National Research Council, Division of Medical Sciences, Ad Hoc Committee on Trauma). Postoperative wound infections: the influence of ultraviolet irradiation on the operating room and of various other factors. Annals of Surgery 1964;160(Suppl 2):1-192.

\section{Berrios-Torres 2017}

Berrios-Torres SI, Umscheid CA, Bratzler DW, Leas B, Stone EC, Kelz RR, et al, Healthcare Infection Control Practices Advisory Committee. Centers for Disease Control and Prevention Guideline for the Prevention of Surgical Site Infection, 2017. JAMA Surgery 2017;152(8):784-91.

\section{Biswas 2005}

Biswas S. Elective inguinal hernia repair with mesh: is there need for antibiotic prophylaxis? A review. World Journal of Surgery 2005;29(7):830-6.

\section{Bluebelle Study Group 2018}

Bluebelle Study Group. Validation of the Bluebelle Wound Healing Questionnaire for assessment of surgical-site infection in closed primary wounds after hospital discharge. British Journal of Surgery 2018;106(3):226-35.

\section{Bratzler 2013}

Bratzler DW, Dellinger EP, Olsen KM, Perl TM, Auwaerter PG, Bolon MK, et al. Clinical practice guidelines for antimicrobial prophylaxis in surgery. Surgical Infections 2013;14(1):73-156.

\section{Cai 2018}

Cai LZ, Foster D, Kethman WC, Weiser TG, Forrester JD. Surgical Site Infections after InguinalHernia Repairs Performed in Lowand Middle-Human Development Index Countries:A Systematic Review. Surgical Infections 2018;19(1):11-20.

\section{Cainzos 1990}

Cainzos M, Lozano F, Balibrea JL, Dávila D, Potel J, Gómez Alonso A, et al. Postoperative infection: multicentric, 
prospective and controlled study [La infección postoperatoria: estudio multicéntrico, prospectivo y controlado]. Cirugia Espanola 1990;48(5):481-90.

\section{Chokshi 2019}

Chokshi A, Sifri Z, Cennimo D, Horng H. Global contributors to antibiotic resistance. Journal of Global Infectious Diseases 2019;11(1):36-42.

\section{Codina 1999}

Codina C, Trilla A, Riera N, Tuset M, Carne X, Ribas J, et al. Perioperative antibiotic prophylaxis in Spanish hospitals: results of a questionnaire survey. Infection Control and Hospital Epidemiology 1999;20(6):436-9.

\section{Condon 1991}

Condon RE, Wittmann DH. The use of antibiotics in general surgery. Current Problems in Surgery 1991;28(12):803-907.

\section{Covidence [Computer program]}

Veritas Health Innovation Covidence. Version accessed April to August 2018. Melbourne, Australia: Veritas Health Innovation. Available at covidence.org.

\section{Dellinger 1994}

Dellinger EP, Gross PA, Barrett TL, Krause PJ, Martone WJ, McGowan JE, et al. Quality standard for antimicrobial prophylaxis in surgical procedures. Clinical Infectious Diseases 1994;18(3):422-7.

\section{Dindo 2004}

Dindo D, Demartines N, Clavien PA. Classification of surgical complications: a new proposal with evaluation in a cohort of 6336 patients and results of a survey. Annals of Surgery 2004;240(2):205-13.

\section{EUCAST System for Antimicrobial Abbreviations}

EUCAST. EUCAST System for Antimicrobial Abbreviations. www.eucast.org/fileadmin/src/media/PDFs/ EUCAST_files/Disk_test_documents/Disk_abbreviations/ EUCAST_system_for_antimicrobial_abbreviations.pdf July 2018.

\section{Fawole 2006}

Fawole AS, Chaparala RP, Ambrose NS. Fate of the inguinal hernia following removal of infected prosthetic mesh. Hernia 2006;10(1):58-61.

\section{Finan 2005}

Finan KR, Vick CC, Kiefe Cl, Neumayer L, Hawn MT. Predictors of wound infection in ventral hernia repair. American Journal of Surgery 2005;190(5):676-81.

\section{Finch 2019}

Finch DA, Misra VA, Hajibandeh S. Open darn repair vs open mesh repair of inguinal hernia: a systematic review and metaanalysis of randomised and non-randomised studies. Hernia 2019;23(3):523-39.

\section{Fitzgibbons 2013}

Fitzgibbons RJ, Ramanan B, Arya S, Turner SA, Li X, Gibbs JO, et al, Investigators of the Original Trial. Long-term results of a randomized controlled trial of a non-operative strategy (watchful waiting) for men with minimally symptomatic inguinal hernias. Annals of Surgery 2013;258(3):508-15.

\section{Fitzgibbons 2015}

Fitzgibbons RJ, Armour Forse R. Groin hernias in adults. New England Journal of Medicine 2015;372(8):756-63.

\section{Gilbert 1989}

Gilbert Al. An anatomic and functional classification for the diagnosis and treatment of inguinal hernia. American Journal of Surgery 1989;157(3):331-3.

\section{GlobalSurg Collaborative 2018}

GlobalSurg Collaborative. Surgical site infection after gastrointestinal surgery in high-income, middle-income, and low-income countries: a prospective, international, multicentre cohort study. Lancet Infectious Diseases 2018;18(5):516-25.

\section{GRADE pro GDT [Computer program]}

McMaster University (developed by Evidence Prime) GRADE proGDT. Version accessed December 2018. Hamilton (ON): McMaster University (developed by Evidence Prime), 2015. Available at gradepro.org.

\section{Haley 1985}

Haley RW, Culver DH, Morgan WM, White JW, Emori TG, Hooton TM. Identifying patients at high risk of surgical wound infection. American Journal of Epidemiology 1985;121(2):206-15.

\section{Heineck 1999}

Heineck I, Ferreira MB, Schenkel EP. Prescribing practice for antibiotic prophylaxis for 3 commonly performed surgeries in a teaching hospital in Brazil. American Journal of Infection Control 1999;27(3):296-300.

\section{HerniaSurge Group 2018}

HerniaSurge Group. International guidelines for groin hernia management. Hernia 2018;22(1):1-165.

\section{Higgins 2016}

Higgins JP, Lasserson T, Chandler J, Tovey, D, Churchill R. Methodological Expectations of Cochrane Intervention Reviews (MECIR). community.cochrane.org/sites/default/files/uploads/ MECIR\%20PRINTED\%20BOOKLET\%20FINAL\%20v1.01.pdf (accessed prior to 15 January 2020).

\section{Higgins 2017}

Higgins JP, Altman DG, Sterne JA, editor(s). Chapter 8: Assessing risk of bias in included studies. In: Higgins JP, Churchill R, Chandler J, Cumpston MS, editor(s), Cochrane Handbook for Systematic Reviews of Interventions version 5.2.0 (updated June 2017). The Cochrane Collaboration, 2017. Available from www.training.cochrane.org/handbook. 


\section{Holmes 1994}

Holmes J, Readman R. A study of wound infections following inguinal hernia repair. Journal of Hospital Infection 1994;28(2):153-6.

\section{Horan 1992}

Horan TC, Gaynes RP, Martone WJ, Jarvis WR, Emori TG. CDC definitions of nosocomial surgical site infections, 1992: a modification of CDC definitions of surgical wound infections. Infection Control and Hospital Epidemiology 1992;13(10):606-8.

\section{Johanet 2011}

Johanet $\mathrm{H}$, Contival N, Coelio Club. Mesh infection after inguinal hernia repair. Journal of Visceral Surgery 2011;148(5):e392-e394.

\section{Kingsnorth 2003}

Kingsnorth A, LeBlanc K. Hernias: inguinal and incisional. Lancet 2003;362(9395):1561-71.

\section{Kurzer 1998}

Kurzer M, Belsham PA, Kark AE. The Lichtenstein repair. Surgical Clinics of North America 1998;78(6):1025-46.

\section{Lagace-Wiens 2012}

Lagace-Wiens $P$, Rubinstein E. Adverse reactions to betalactam antimicrobials. Expert Opinion on Drug Safety 2012;11(3):38199.

\section{Leaper 1998}

Leaper DJ. Use of antibiotic prophylaxis in clean nonimplant wounds. Journal of Antimicrobial Chemotherapy 1998;41(5):501-4.

\section{Lichtenstein 1989}

Lichtenstein IL, Shulman AG, Amid PK, Montllor MM. The tension-free hernioplasty. American Journal of Surgery 1989;157(2):188-93

\section{Mandell 2010}

Mandell GL, Bennett JE, Dolin R, editors. Principles and Practice of Infectious Diseases. 7th edition. Churchill Livingstone Elsevier, 2010.

\section{Mangram 1999}

Mangram AJ, Horan TC, Pearson ML, Silver LC, Jarvis WR, The Hospital Infection Control Practices Advisory Committee. Guideline for prevention of surgical site infection, 1999. Infection Control and Hospital Epidemiology 1999;20(4):247-78.

\section{Mann 1998}

Mann DV, Prout J, Havranek E, Gould S, Darzi A. Late-onset deep prosthetic infection following mesh repair of inguinal hernia. American Journal of Surgery 1998;176(1):12-4.

\section{Miserez 2014}

Miserez M, Peeteres E, Aufenacker T, Bouillot JL, Campanelli G, Conze J, et al. Update with level 1 studies of the European Hernia Society guidelines on the treatment of inguinal hernia in adult patients. Hernia 2014;18(2):151-63.

\section{Mozillo 1988}

Mozillo N, Greco D, Pescini A, Formato A. Chemoprophylaxis in the surgical ward: results of a national survey in Italy. European Journal of Epidemiology 1988;4(3):357-9.

\section{Ortega 2012}

Ortega G, Rhee DS, Papandria DJ, Yang J, Ibrahim AM, Shore AD, et al. An evaluation of surgical site infections by wound classification system using the ACS-NSQIP. Journal of Surgical Research 2012;174(1):33-8.

\section{Page 1993}

Page CP, Bohnen JMA, Fletcher JR, MacManus AT, Solomkin JS, Wittmann DH. Antimicrobial prophylaxis for surgical wounds. Guidelines for clinical care. Archives of Surgery 1993;128(1):79-88.

\section{Porcu 1996}

Porcu A, Noya G, Dessanti A, Niolu P, Cottu P, Castiglia P, et al. A new approach to the problem of surgical wound infections in clean operations. Minerva Chirurgica 1996;51(9):691-6.

\section{Ranaboldo 1993}

Ranaboldo CJ, Karran SE, Bailey S, Karran SJ. Antimicrobial prophylaxis in clean surgery: hernia repair. Journal of Antimicrobial Chemotherapy 1993;31(Suppl B):35-41.

\section{Rehman 2012}

Rehman S, Khan S, Pervaiz A, Perry EP. Recurrence of inguinal herniae following removal of infected prosthetic meshes: a review of the literature. Hernia 2012;16(2):123-6.

\section{Review Manager 2014 [Computer program]}

Nordic Cochrane Centre, The Cochrane Collaboration Review Manager 5 (RevMan 5). Version 5.3. Copenhagen: Nordic Cochrane Centre, The Cochrane Collaboration, 2014.

\section{Robbins 1998}

Robins AW, Rutkow IM. Mesh plug repair and groin hernia surgery. Surgical Clinics of North America 1998;78(6):1007-23.

\section{Rodriguez 2005}

Rodriguez-Cuellar E, Villeta R, Ruiz P, Alcalde J, Landa JI, Porrero JL, et al. National project for the management of clinical processes. Surgical treatment of inguinal hernia [Proyecto nacional para la gestión clínica de procesos asistenciales. Tratamiento quirúrgico de la hernia inguinal]. Cirugia Espanola 2005;77(4):194-202.

\section{Rutkow 1993}

Rutkow IM, Robbins AW. 'Tension-free' inguinal herniorrhaphy: a preliminary report on the 'mesh-plug' technique. Surgery 1993;114(1):3-8.

\section{Rutkow 2003}

Rutkow IM. Demographic and socio-economic aspects of hernia repair in the United States in 2003. Surgical Clinics of North America 2003;83(5):1045-51. 


\section{Simons 2009}

Simons MP, Aufenacker T, Bay-Nielsen M, Bouillot JL, Campanelli G, Conze J, et al. European Hernia Society guidelines on the treatment of inguinal hernia in adult patients. Hernia 2009;13(4):343-403.

\section{Stephenson 2003}

Stephenson BM. Complications of open groin hernia. Surgical Clinics of North America 2003;83(5):1255-78.

\section{Wittmann 1995}

Wittmann DH, Schein M, Condon RE. Antibiotic prophylaxis in abdominal wall hernia surgery: never, always, or selectively? Problems in General Surgery 1995;12(1):47-55.

\section{Woods 1998}

Woods RK, Dellinger EP. Current guidelines for antibiotic prophylaxis of surgical wounds. American Family Physician 1998;58(11):2731-40.

\section{Zollinger 2003}

Zollinger RM. Classification systems for groin hernias. Surgical Clinics of North America 2003;83(5):1053-63.

\section{References to other published versions of this review}

\section{Sanchez-Manuel 2001}

Sánchez-Manuel FJ, Seco-Gil JL, Lozano-García J. Antibiotic prophylaxis and hernia repair. Systematic quantitative review results [Profilaxis antibiótica y reparación herniaria. Resultado de una revisión sistemática cuantitativa.]. Enfermedades Infecciosas y Microbiologia Clinica 2001;19(3):107-13.

\section{Sanchez-Manuel 2003}

Sánchez-Manuel FJ, Seco-Gil JL. Antibiotic prophylaxis for hernia repair. Cochrane Database of Systematic Reviews 2003, Issue 2. Art. No: CD003769. [DOI: 10.1002/14651858.CD003769]

\section{Sanchez-Manuel 2004}

Sánchez-Manuel FJ, Seco-Gil JL. Antibiotic prophylaxis for hernia repair. Cochrane Database of Systematic Reviews 2004, Issue 4. Art. No: CD003769. [DOI: 10.1002/14651858.CD003769.pub2]

\section{Sanchez-Manuel 2007}

Sánchez-Manuel FJ, Lozano-García J, Seco-Gil JL. Antibiotic prophylaxis for hernia repair. Cochrane Database of Systematic Reviews 2007, Issue 3. Art. No: CD003769. [DOI: 10.1002/14651858.CD003769.pub3]

\section{Sanchez-Manuel 2012}

Sánchez-Manuel FJ, Lozano-García J, Seco Gil JL. Antibiotic prophylaxis for hernia repair. Cochrane Database of Systematic Reviews 2012, Issue 2. Art. No: CD003769. [DOI: 10.1002/14651858.CD003769.pub4]

* Indicates the major publication for the study

\section{CHARACTERISTICS OF STUDIES}

Characteristics of included studies [ordered by study ID]

\section{Al-Fatah 2011}

\section{Study characteristics}

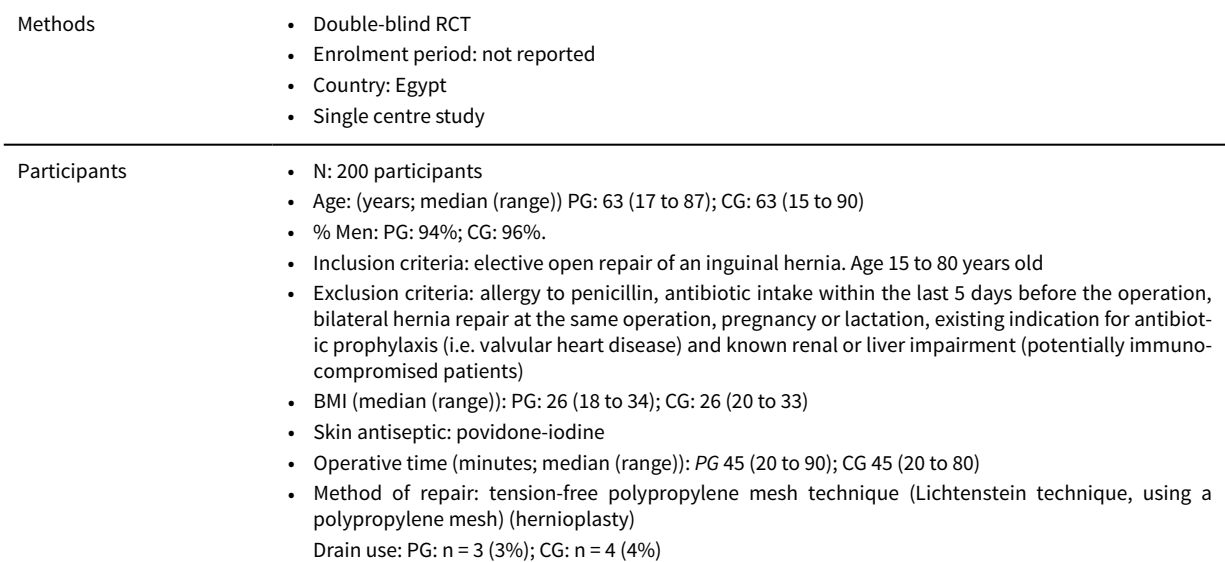


- ASA class: PG: ASAI- $n=75$ (75\%); ASAll $n=22(22 \%)$; ASAIII $n=3(3 \%)$; CG: ASAI $n=71(71 \%)$; ASAll $n$ $=27(27 \%) ;$ ASAIII $n=2(2 \%)$

- Anaesthesia method: spinal

- Hospitalisation method: inpatient

- Length of hospital stay: 1 day (patients were discharged 1 day after surgery)

- Surgeon: a single surgeon

\begin{tabular}{ll}
\hline - Antibiotic prophylactic group (PG): \\
- N: 100 patients \\
- antibiotic: amoxicillin-clavulanic acid \\
- dose: $1.2 \mathrm{~g}$ \\
- number of doses: 1 \\
- route of administration: intravenous \\
- Control group (CG): \\
- N: 100 patients \\
- substance: equal volume of sterile saline \\
\hline - Wound infection definition: 1 ) purulent discharge (or serosanguineous with positive culture) or 2) \\
spreading erythema indicative of cellulitis or 3) wound breakdown/dehiscence with clinical evidence \\
of infection. \\
- Follow-up: 1 week and 1 month post operation \\
- SSSI $\mathrm{N}$ infected/N patients (\%) PG: $3 / 100$ (3\%); CG: $5 / 100$ (5\%) \\
- DSSI: not reported (reported: "not necessary to remove any mesh") \\
- Adverse effects: not reported \\
- Staphylococcus aureus, Staphylococcus epidermidis and Streptococcus haemolyticus bacteria were \\
isolated from the wound infections \\
- Participants aged < 18 years old were included in this study \\
- No funding reported
\end{tabular}

\section{Risk of bias}

\begin{tabular}{|c|c|c|}
\hline Bias & Authors' judgement & Support for judgement \\
\hline $\begin{array}{l}\text { Random sequence genera- } \\
\text { tion (selection bias) }\end{array}$ & Low risk & $\begin{array}{l}\text { Quote: “...list with code numbers generated by the Arcus Quiqstat randomisa- } \\
\text { tion program" }\end{array}$ \\
\hline $\begin{array}{l}\text { Allocation concealment } \\
\text { (selection bias) }\end{array}$ & Unclear risk & $\begin{array}{l}\text { Not specified whether envelopes were sequentially numbered and opaque. } \\
\text { Quote: "Patients were randomised .... using numbered sealed envelopes" and } \\
\text { "The list with code numbers generated by the Arcus Quiqstat randomisation } \\
\text { program was kept by a secretary who was not involved in the treatment of the } \\
\text { patients at any stage and was opened at the end of the trial for analysis of the } \\
\text { results." }\end{array}$ \\
\hline $\begin{array}{l}\text { Blinding of participants } \\
\text { and personnel (perfor- } \\
\text { mance bias) } \\
\text { All outcomes }\end{array}$ & Low risk & $\begin{array}{l}\text { Quote: "The agent (antibiotic or placebo) was prepared by the anaesthetic } \\
\text { nurse and neither the surgeon or the patient was aware of the randomisation } \\
\text { arm" }\end{array}$ \\
\hline $\begin{array}{l}\text { Blinding of outcome as- } \\
\text { sessment (detection bias) } \\
\text { All outcomes }\end{array}$ & Low risk & $\begin{array}{l}\text { Quote: "The list with code numbers generated by the Arcus Quiqstat randomi- } \\
\text { sation program was kept by a secretary who was not involved in the treatment } \\
\text { of the patients at any stage and was opened at the end of the trial for analysis } \\
\text { of the results." }\end{array}$ \\
\hline
\end{tabular}


Quote: "They were followed up as outpatients at 1 week and 1 month after the operation by the surgeon." ; "...neither the surgeon or the patient was aware of the randomisation arm"

\begin{tabular}{lll}
\hline $\begin{array}{l}\text { Incomplete outcome data } \\
\text { (attrition bias) } \\
\text { All outcomes }\end{array}$ & Low risk & No missing data. \\
\hline $\begin{array}{l}\text { Selective reporting (re- } \\
\text { porting bias) }\end{array}$ & Low risk & $\begin{array}{l}\text { The study protocol was not available but report includes all expected out- } \\
\text { comes, including specification of SSSI. }\end{array}$ \\
\hline Other bias & Low risk & The study appears to be free of other sources of bias. \\
\hline
\end{tabular}

\section{Andersen 1980}

\section{Study characteristics}

\begin{tabular}{|c|c|}
\hline Methods & $\begin{array}{l}\text { - Triple-blind RCT } \\
\text { - Country: Denmark } \\
\text { - Enrolment period: April } 1973 \text { to October } 1975 \\
\text { - Single centre study }\end{array}$ \\
\hline Participants & $\begin{array}{l}\text { - N: } 285 \text { participants } \\
\text { - Age (years; mean (range)): PG: } 54 \text { to } 56 \text { years (range } 21 \text { to } 82 \text { ); CG: } 55 \text { years (range } 20 \text { to 82) } \\
\text { - } \% \text { Men: PG: } 72 \% \text {; CG: } 73 \% \\
\text { - Inclusion criteria: direct hernia } \\
\text { - Exclusion criteria: allergy to penicillin } \\
\text { - BMI: not reported. } \\
\text { - Skin antiseptic: not reported. } \\
\text { - Operative time: not reported. } \\
\text { - Method of repair: Mc Vay technique (herniorrhaphy) } \\
\text { - Drain use: removed at postoperative day } 4 \\
\text { - ASA class: not reported } \\
\text { - Anaesthesia method: not reported } \\
\text { - Hospitalisation method: inpatient } \\
\text { - Length of hospital stay: not reported } \\
\text { - Surgeon: not reported }\end{array}$ \\
\hline Interventions & $\begin{array}{l}\text { - Prophylactic group (PG): } \\
\text { - N: } 135 \\
\text { - Group 1: }(n=59) \text { Ampicillin sodium powder } 1 \mathrm{~g} \text { in the subfascial layers and silk sutures in the fascia } \\
\text { and peritoneum } \\
\text { - Group 2: }(n=76) \text { As Group } 1 \text { but polyglycolic acid instead silk sutures } \\
\text { - Timing of administration: before closure fascial } \\
\text { - Control group }(C G): \\
\text { - N: } 150 \\
\text { - Group } 3:(n=86) \text { No antibiotic therapy and silk sutures } \\
\text { - Group } 4:(n=64) \text { No antibiotic therapy and polyglycolic acid sutures }\end{array}$ \\
\hline
\end{tabular}


- DSSI: not reported

- Adverse effects: "none of the patients had an allergic reaction to ampicillin"

Notes
- The study was realised in participants who underwent various techniques other that hernia repair, but it was possible to extract the information relating to the patients underwent to hernia repair.

- Funding was not reported

\section{Risk of bias}

\begin{tabular}{|c|c|c|}
\hline Bias & Authors' judgement & Support for judgement \\
\hline \multirow[t]{2}{*}{$\begin{array}{l}\text { Random sequence genera- } \\
\text { tion (selection bias) }\end{array}$} & Unclear risk & $\begin{array}{l}\text { Insufficient information available on how "triple-blind random allocation de- } \\
\text { sign" was performed }\end{array}$ \\
\hline & & $\begin{array}{l}\text { Quote: "patients ... who had a direct hernia or gallstones in a gallbladder } \\
\text { (shown not to be infected on macroscopic examination) were assigned at the } \\
\text { end of the operation to one of the following four regimens: one gram of ampi- } \\
\text { cillin powder ... or no antibiotic therapy" }\end{array}$ \\
\hline $\begin{array}{l}\text { Allocation concealment } \\
\text { (selection bias) }\end{array}$ & Unclear risk & $\begin{array}{l}\text { Quote: "...were assigned at the end of the operation to one of the following } \\
\text { four regimens:..." }\end{array}$ \\
\hline $\begin{array}{l}\text { Blinding of participants } \\
\text { and personnel (perfor- } \\
\text { mance bias) } \\
\text { All outcomes }\end{array}$ & Low risk & No information available, but performance bias is unlikely in this setting. \\
\hline $\begin{array}{l}\text { Blinding of outcome as- } \\
\text { sessment (detection bias) } \\
\text { All outcomes }\end{array}$ & Unclear risk & No information available \\
\hline \multirow{2}{*}{$\begin{array}{l}\text { Incomplete outcome data } \\
\text { (attrition bias) } \\
\text { All outcomes }\end{array}$} & Low risk & $\begin{array}{l}2 \text { patients (out of total of } 400 \text { patients) were lost in follow-up. Available case } \\
\text { analysis was performed. }\end{array}$ \\
\hline & & $\begin{array}{l}\text { The proportion of missing outcomes was considered not enough to have had a } \\
\text { clinically relevant impact on the intervention effect estimate. }\end{array}$ \\
\hline $\begin{array}{l}\text { Selective reporting (re- } \\
\text { porting bias) }\end{array}$ & Low risk & $\begin{array}{l}\text { Study protocol was not registered, but report contains all expected outcomes, } \\
\text { including specification of SSSI }\end{array}$ \\
\hline Other bias & Low risk & The study appears to be free of other sources of bias \\
\hline
\end{tabular}

\section{Aufenacker 2004}

\section{Study characteristics}

\begin{tabular}{ll}
\hline Methods & Double-blind RCT \\
& - Country: the Netherlands \\
& - Enrolment period: November 1998 to May 2003 \\
& Multicentre (4) study \\
\hline Participants & N: 1040 participants \\
& - Age: (years; mean \pm SD) PG: $58.3 \pm 12.9 ;$ CG: $58.2 \pm 13.2$ \\
& $\%$ Men: PG: $95.6 \% ; C G: 97.0 \%$
\end{tabular}


- Inclusion criteria: primary uni- or bilateral inguinal hernia and an indication for Lichtenstein hernia repair

- Exclusion criteria: age under 35, the need for antibiotics for a different reason, immunosuppressive disease (diabetes mellitus, malignancy, HIV) or medication (glucocorticoid therapy), allergy to the given antibiotic, recurrent hernia, or the inability to get an informed consent.

- BMI: not reported

- Skin antiseptic: PG: povidone iodine $98 \%$ of patients; CG: povidone iodine $98.4 \%$ of patients

- Operative time (minutes, median (IQR)) PG: 40 (30 to 50) / CG 40 (28 to 51)

- Method of repair: Lichtenstein technique with polypropylene mesh (hernioplasty).

- Drain use: PG: $\mathrm{n}=11(2.2 \%) ; \mathrm{CG}: \mathrm{n}=4(0,8 \%)$

- ASA class: not reported

- Anaesthesia method: PG: local $n=10(2 \%)$; spinal $n=180(35.8 \%)$; general $n=311(61 \%)$; unknown $n$ $=2(0.4 \%)$; CG: local $n=7(1.4 \%)$; spinal $n=191(37.8 \%)$; general $n=330(60 \%)$; unknown $n=4(0.8 \%)$.

- Hospitalisation method: PG: outpatients $n=231$ (46.1\%); CG: outpatients $n=232(45.9 \%)$

- Length of hospital stay: not reported

- Surgeon: PG: resident $n=212(42.1 \%)$, certified surgeon $n=291(57.9 \%)$; CG resident $n=225(44.6 \%)$, certified surgeon $n=280(55.4 \%)$

- Prophylactic group (PG):

- $\mathrm{N}: 503$

- antibiotic: cefuroxime

- dose: $1500 \mathrm{mg}$

- number of doses: 1

- route of administration: intravenous

- timing of administration: induction of anaesthesia. The exact timing of administering was not standardised, thereby copying daily practice.

- Control group (CG):

- N: 505

- substance: equal volume of sterile saline.

\begin{tabular}{|c|c|}
\hline Outcomes & $\begin{array}{l}\text { - Wound infection definition: CDC criteria. } \\
\text { - Follow-up: } 1,2,12 \text { weeks } \\
\text { - SSSI N infected/N patients (\%) PG: 7/503 (1.4\%); CG: 7/505 (1.4\%) } \\
\text { - DSSI N infected/N patients (\%) PG: } 1 / 503(0.2 \%) \text {; CG 2/505 (0.4\%) } \\
\text { - Adverse effects: not reported }\end{array}$ \\
\hline Notes & $\begin{array}{l}\text { - Staphylococcus aureus, Enterococcus faecalis, Corynebacterium, Streptococcus group G/Aspergillus fu- } \\
\text { migatus and skin bacteria were isolated from the wound infection } \\
\text { - Funding was not reported. }\end{array}$ \\
\hline
\end{tabular}

\section{Risk of bias}

\begin{tabular}{lll}
\hline Bias & Authors' judgement & Support for judgement \\
\hline $\begin{array}{l}\text { Random sequence genera- } \\
\text { tion (selection bias) }\end{array}$ & Low risk & $\begin{array}{l}\text { Quote: “....according to a computer generated list in blocks of } 10 \text { patients with } \\
\text { stratification for each hospital." }\end{array}$ \\
\hline $\begin{array}{l}\text { Allocation concealment } \\
\text { (selection bias) }\end{array}$ & Low risk & $\begin{array}{l}\text { Quote: "The patients were double-blinded randomly assigned to either intra- } \\
\text { venous placebo or antibiotic prophylaxis. A pharmacist carried out random- } \\
\text { ization....” and "A pharmacist prepared the trial medication under laminar air- } \\
\text { flow condition, and it was packed in nontransparent material to exclude opti- } \\
\text { cal differences. The anesthesiologist administered the trial medication at the } \\
\text { induction of anesthesia." }\end{array}$ \\
\hline
\end{tabular}


Quote: "A pharmacist prepared the trial medication under laminar airflow condition, and it was packed in nontransparent material to exclude optical differences. The anesthesiologist administered the trial medication at the induction of anesthesia." sessment (detection bias)

All outcomes

Quote: "In most cases, the surgeon who performed the operation did not perform the follow-up. In case of missing observations, the patients were contacted and a standardized telephone interview was performed."
Blinding of outcome as- Low risk

In the few cases that the surgeon who performed the operation also performed the follow-up, it should be kept in mind that Quote: " trial medication ...was packed in nontransparent material to exclude optical differences" and that therefore the surgeon was unaware whether participant was part of intervention or control group.

Incomplete outcome data Low risk (attrition bias)

Missing data were balanced between intervention groups. PG $n=17(17 / 520=$ $3.2 \%)$ and $C G n=15(15 / 520=2.9 \%)$. The proportion of missing outcomes was considered not enough to have had a clinically relevant impact on the intervention effect estimate

Available case analysis was performed

1 death in placebo-group due to operation-related complication (Quote: "One patient died of pulmonary complications and a bleeding gastric ulcer".)

\begin{tabular}{lll}
\hline $\begin{array}{l}\text { Selective reporting (re- } \\
\text { porting bias) }\end{array}$ & Low risk & $\begin{array}{l}\text { Study protocol was not registered, but report contains all expected outcomes, } \\
\text { including specification of SSSI and DSSI }\end{array}$ \\
\hline Other bias & Low risk & The study appears to be free of other sources of bias \\
\hline
\end{tabular}

\section{Bidhur 2013}

\section{Study characteristics}

\begin{tabular}{ll}
\hline Methods & RCT \\
& - Country: Nepal \\
- Enrolment period: April 2008 to June 2009 \\
- Single centre study.
\end{tabular}

Participants

- $\mathrm{N}: 60$ participants

- Age (years; mean \pm SD): overall population: $38.5 \pm 17.7$ (range: 19 to 90 years); age < 30 years PG: $\mathrm{n}=$ $12(40 \%) ;$ CG: $n=11(36.7 \%)$

- \% Men: PG: $100 \%$; CG: $96.7 \%$

- Inclusion criteria: age > 18 years, primary inguinal hernia

- Exclusion criteria: recurrent, strangulated, bilateral inguinal hernia, femoral hernia, age $<18$ years, allergy to Cefuroxime, systemic or advanced diseases like diabetes mellitus, liver or renal impairment, patients on steroids or antibiotic within a week before surgery, or pregnant or lactating women.

- BMI: not reported

- Skin antiseptic: not reported

- Operative time (minutes, mean $\pm \mathrm{SD}$ ): overall population: $51.3 \pm 9.8$ (range 40 to $75 \mathrm{~min}$ ); time $<50$ min: PG: $n=19(63 \%) ; C G: n=15(50 \%)$

- Method of repair: Lichtenstein technique (hernioplasty) with monofilament polypropylene mesh (hernioplasty)

- Drain use: not reported

ASA class: not reported 
- Anaesthesia method: local $(n=42)$ or spinal (regional, $n=18$ )

- Hospitalisation method: outpatient (for local anaesthesia) or inpatient (for spinal anaesthesia)

- Length of hospital stay: 1 day for spinal anaesthesia, day surgery for local anaesthesia

- Surgeon: qualified surgeon or resident under supervision of faculty (not specified per treatment group)

\begin{tabular}{|c|c|}
\hline Interventions & $\begin{array}{l}\text { - Prophylactic group (PG): } \\
\text { - } \mathrm{N}: 30 \\
\text { - antibiotic: cefuroxime } \\
\text { - dose: } 1500 \mathrm{mg} \\
\text { - number of doses: } 1 \\
\text { - route of administration: intravenous } \\
\text { - timing of administration: induction of anaesthesia (spinal anaesthesia) or just before incision (local } \\
\text { anaesthesia). } \\
\text { - Control group (CG): } \\
\text { - } \mathrm{N}: 30 \\
\text { - substance: equal volume of sterile saline. }\end{array}$ \\
\hline
\end{tabular}

\begin{tabular}{ll}
\hline Outcomes & Wound infection definition: CDC criteria. \\
- Follow-up: 7 to 9 days, 28 to 42 days post operation \\
- SSSI N infected/N patients (\%) PG: $0 / 30(0 \%) ; C G: 1 / 30(3.3 \%)$ \\
- DSSI: not reported \\
- Adverse effects: not reported
\end{tabular}

Notes Reported is: no conflict of interest

\section{Risk of bias}

\begin{tabular}{|c|c|c|}
\hline Bias & Authors' judgement & Support for judgement \\
\hline $\begin{array}{l}\text { Random sequence genera- } \\
\text { tion (selection bias) }\end{array}$ & Low risk & $\begin{array}{l}\text { Quote: "Randomization was done as listed by randomization technique in Mi- } \\
\text { crosoft Excel Programme" }\end{array}$ \\
\hline $\begin{array}{l}\text { Allocation concealment } \\
\text { (selection bias) }\end{array}$ & Unclear risk & No information available. \\
\hline $\begin{array}{l}\text { Blinding of participants } \\
\text { and personnel (perfor- } \\
\text { mance bias) } \\
\text { All outcomes }\end{array}$ & Low risk & No information available, but performance bias is unlikely in this setting. \\
\hline $\begin{array}{l}\text { Blinding of outcome as- } \\
\text { sessment (detection bias) } \\
\text { All outcomes }\end{array}$ & Unclear risk & No information available. \\
\hline $\begin{array}{l}\text { Incomplete outcome data } \\
\text { (attrition bias) } \\
\text { All outcomes }\end{array}$ & Low risk & No missing outcome data. \\
\hline $\begin{array}{l}\text { Selective reporting (re- } \\
\text { porting bias) }\end{array}$ & Low risk & $\begin{array}{l}\text { Study protocol was not registered, but report contains all expected outcomes, } \\
\text { including specification of SSSI. }\end{array}$ \\
\hline Other bias & Low risk & Study appears to be free from other sources of bias. \\
\hline
\end{tabular}




\section{Study characteristics}

\begin{tabular}{|c|c|c|}
\hline Methods & \multicolumn{2}{|c|}{$\begin{array}{l}\text { - Double-blind RCT } \\
\text { - Enrolment period: October } 1997 \text { - not reported } \\
\text { - Country: Spain } \\
\text { - Single centre study }\end{array}$} \\
\hline Participants & \multicolumn{2}{|c|}{ 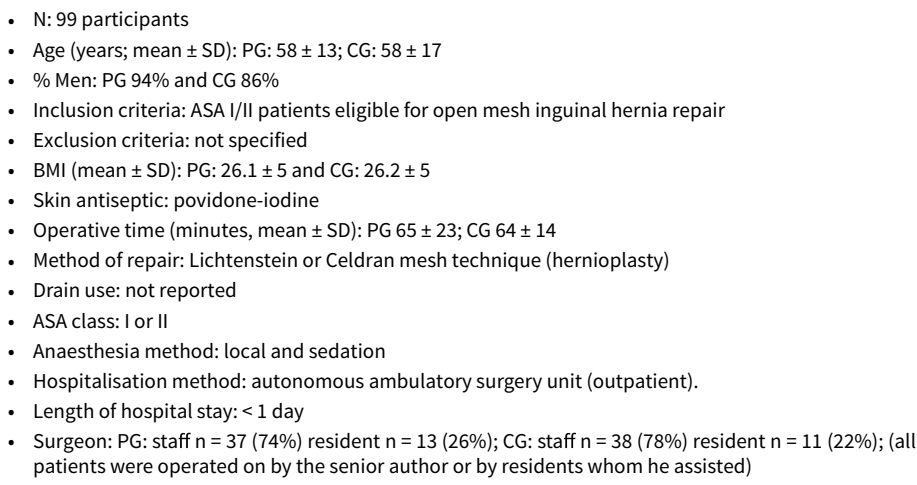 } \\
\hline Interventions & \multicolumn{2}{|c|}{$\begin{array}{l}\text { Prophylactic group (PG): } \\
\text { - } \mathrm{N}: 50 \\
\text { - antibiotic: cefazolin } \\
\text { - dose: } 1 \mathrm{~g} \\
\text { - number of doses: } 1 \\
\text { - route of administration: parenteral (not specified) } \\
\text { - timing of administration: } 30 \text { minutes before the incision } \\
\text { - Control group (CG): } \\
\text { - } \mathrm{N}: 49 \\
\text { - substance: placebo (not specified) }\end{array}$} \\
\hline Outcomes & \multicolumn{2}{|c|}{$\begin{array}{l}\text { - Wound infection definition: CDC criteria } \\
\text { - Follow-up: } 1 \text { week, } 1,3,6 \text { months, } 1 \text { and } 2 \text { years } \\
\text { - SSSI N infected/N patients (\%) PG: 0/50 (0\%); CG: } 4 / 49(8.2 \%) \\
\text { - DSSI N infected/N patients (\%) PG: 0/50 (0\%); CG: 0/49 (0\%) (reported: "not necessary to remove any } \\
\text { mesh") } \\
\text { - Adverse effects: not reported }\end{array}$} \\
\hline Notes & $\begin{array}{l}\text { - Staphylococcus aur } \\
\text { - Funding was not re }\end{array}$ & $\begin{array}{l}\text { us and Klebsiella oxytoca bacteria were isolated from the wound infections } \\
\text { orted. }\end{array}$ \\
\hline \multicolumn{3}{|l|}{ Risk of bias } \\
\hline Bias & Authors' judgement & Support for judgement \\
\hline $\begin{array}{l}\text { Random sequence genera- } \\
\text { tion (selection bias) }\end{array}$ & Low risk & Quote: "A list of random numbers was generated to assign the treatment." \\
\hline
\end{tabular}


Celdran 2004 (Continued)

Allocation concealment Unclear risk Insufficient information available (selection bias)

Blinding of participants

Low risk

No information available, but performance bias is unlikely in this setting and personnel (perfor-

mance bias)

All outcomes

\begin{tabular}{|c|c|c|}
\hline $\begin{array}{l}\text { Blinding of outcome as- } \\
\text { sessment (detection bias) }\end{array}$ & Low risk & $\begin{array}{l}\text { Quote: "All patients were examined } 1 \text { week, } 1,3 \text {, and } 6 \text { months, and } 1 \text { and } 2 \\
\text { years postoperatively by trained impartial surgeon." }\end{array}$ \\
\hline
\end{tabular}
All outcomes

\begin{tabular}{lll}
\hline $\begin{array}{l}\text { Incomplete outcome data } \\
\text { (attrition bias) } \\
\text { All outcomes }\end{array}$ & Low risk & No missing outcome data. \\
\hline $\begin{array}{l}\text { Selective reporting (re- } \\
\text { porting bias) }\end{array}$ & Low risk & $\begin{array}{l}\text { The study protocol was not registered, but report includes all expected out- } \\
\text { comes, including specification of SSSI and DSSI }\end{array}$ \\
\hline Other bias & High risk & $\begin{array}{l}\text { Study was terminated early after interim analysis, and therefore there might } \\
\text { be risk of bias for increased benefit. } \\
\text { Quote: "Although it was designed to include a higher number of patients, the } \\
\text { interim analysis, performed after the first } 91 \text { patients had been included, rec- } \\
\text { ommended ending the study due to ethical reasons." }\end{array}$
\end{tabular}

Ergul 2011

\section{Study characteristics}

\begin{tabular}{|c|c|}
\hline Methods & $\begin{array}{l}\text { - Double-blind RCT } \\
\text { - Enrolment period: July } 2008 \text { to October } 2010 \\
\text { - Country: Turkey } \\
\text { - Single centre }\end{array}$ \\
\hline Participants & 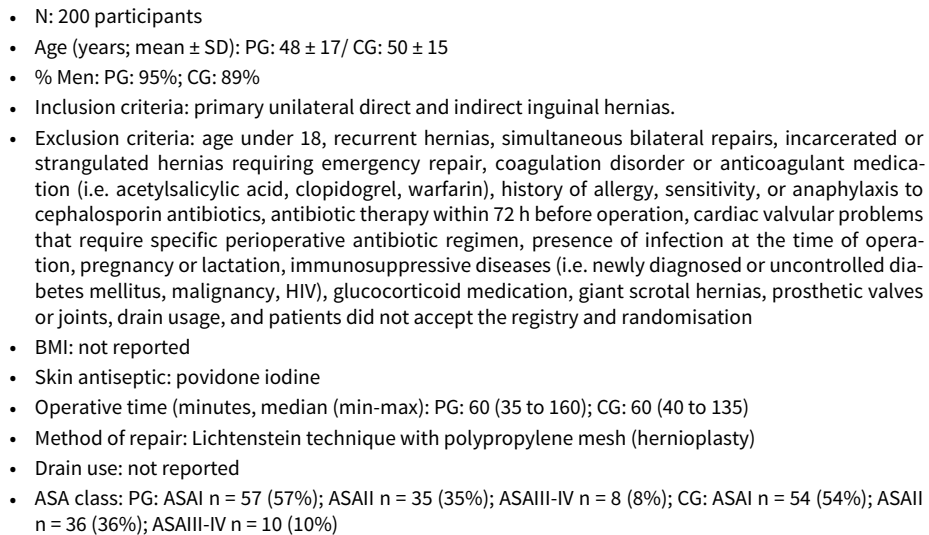 \\
\hline
\end{tabular}


- Anaesthesia method: PG: general $n=48(48 \%)$, regional $n=39(39 \%)$, local $n=12(12 \%)$; CG: general $n=53(53 \%)$, regional $n=36(36 \%)$, local $n=11(11 \%)$

- Hospitalisation method: inpatient

- Length of hospital stay (days, median (min-max)): PG: 1 (1 to 1 ); CG: 1 (1 to 4 )

- Surgeon: supervised residents or staff surgeons

\begin{tabular}{|c|c|}
\hline Interventions & $\begin{array}{l}\text { Prophylactic group }(\mathrm{PG}) \text { : } \\
\text { - } \mathrm{N}: 100 \\
\text { - antibiotic: cefazolin } \\
\text { - dose: } 1 \mathrm{~g} \\
\text { - number of doses: } 1 \\
\text { - timing of administration: when the patient entered the operating room or, at the latest, at the in- } \\
\text { duction of anaesthesia. } \\
\text { - } \mathrm{N} \text { : } 100 \\
\text { - substrance: equal volume of sterile saline. }\end{array}$ \\
\hline Outcomes & $\begin{array}{l}\text { - Wound infection definition: CDC criteria } \\
\text { - Follow-up: POD } 1 \text { and } 3 ; 3,5,6 \text { and } 30 \text { days after discharge } \\
\text { - SSSI N infected/N patients (\%): PG: } 5 / 100(5 \%) ; C G: 7 / 100(7 \%) \\
\text { - DSSI N infected/N patients (\%): PG: } 0 / 100(0 \%) ; C G: 0 / 100(0 \%) \\
\text { - Adverse effects: perioperative allergic reaction in } 1 \text { patient, probably due to allergy to anaesthesia as } \\
\text { patient belonged to control group. }\end{array}$ \\
\hline Notes & $\begin{array}{l}\text { - Staphylococcus aureus bacteria were isolated from the wound infections } \\
\text { - Reported: no conflict of interest }\end{array}$ \\
\hline
\end{tabular}

\section{Risk of bias}

\begin{tabular}{|c|c|c|}
\hline Bias & Authors' judgement & Support for judgement \\
\hline $\begin{array}{l}\text { Random sequence genera- } \\
\text { tion (selection bias) }\end{array}$ & Low risk & $\begin{array}{l}\text { Quote: "Patients were randomized by use of sealed envelopes which included } \\
\text { equal numbers of patients to be randomized either to the control arm or to the } \\
\text { antibiotic prophylaxis arm." }\end{array}$ \\
\hline $\begin{array}{l}\text { Allocation concealment } \\
\text { (selection bias) }\end{array}$ & Unclear risk & $\begin{array}{l}\text { Not specified whether envelopes were sequentially numbered and opaque. } \\
\text { Quote: "Patients were randomized by use of sealed envelopes which included } \\
\text { equal numbers of patients to be randomized either to the control arm or to the } \\
\text { antibiotic prophylaxis arm." }\end{array}$ \\
\hline $\begin{array}{l}\text { Blinding of participants } \\
\text { and personnel (perfor- } \\
\text { mance bias) } \\
\text { All outcomes }\end{array}$ & Low risk & $\begin{array}{l}\text { Quote: The anaesthesiologist administered the trial medication (antibiotic or } \\
\text { sterile saline in coded syringes) }\end{array}$ \\
\hline $\begin{array}{l}\text { Blinding of outcome as- } \\
\text { sessment (detection bias) } \\
\text { All outcomes }\end{array}$ & Low risk & $\begin{array}{l}\text { Quote: "The surgeon who performed the follow-up frequently was not the sur- } \\
\text { geon who performed the operation." }\end{array}$ \\
\hline $\begin{array}{l}\text { Incomplete outcome data } \\
\text { (attrition bias) } \\
\text { All outcomes }\end{array}$ & Low risk & $\begin{array}{l}\text { No missing outcome data. } \\
\text { Quote: "No patient was lost to follow-up." }\end{array}$ \\
\hline
\end{tabular}


Ergul 2011 (Continued)

Selective reporting (re- Low risk

Quote: "The trial was registered on http://www.controlled-trials.com porting bias) (ISRCTN85660082)" and all of the prespecified outcomes that were of interest have been reported in the prespecified way.

Other bias

Low risk

The study appears to be free of other sources of bias

Evans 1973

\section{Study characteristics}

\begin{tabular}{|c|c|}
\hline Methods & $\begin{array}{l}\text { - RCT } \\
\text { - Enrolment period: not reported } \\
\text { - Country: UK } \\
\text { - Single centre }\end{array}$ \\
\hline Participants & $\begin{array}{l}\text { - } \mathrm{N}: 97 \text { participants } \\
\text { - Age: not reported } \\
\text { - } \% \text { Men: not reported } \\
\text { - Inclusion criteria: Several types of clean or contaminated surgeries, age > } 16 \text { years } \\
\text { - Exclusion criteria: not specified } \\
\text { - BMI: not reported } \\
\text { - Skin antiseptic: not reported } \\
\text { - Operative time: } \text { not reported } \\
\text { - Method of repair: not reported, herniorrhaphy } \\
\text { - Drain use: not reported } \\
\text { - ASA class: not reported } \\
\text { - Anaesthesia method: not reported } \\
\text { - Hospitalisation method: not reported (most likely inpatient) } \\
\text { - Length of hospital stay: not reported } \\
\text { - Surgeon: not reported }\end{array}$ \\
\hline Interventions & $\begin{array}{l}\text { Prophylactic group (PG): } \\
\mathrm{N}: 48 \\
\text { - antibiotic: cephaloridine } \\
\text { - dose: } 1 \mathrm{~g} \\
\text { - number of doses: multiple (3) } \\
\text { - route of administration: intravenous at induction and intramuscular after. } \\
\text { - timing of administration: induction and at } 5 \text { and } 12 \mathrm{~h} \text { later. } \\
\text { Control group (CG): } \\
\text { - } \mathrm{N}: 49 \\
\text { - substance: nothing }\end{array}$ \\
\hline Outcomes & $\begin{array}{l}\text { - Wound infection definition: presence of pus which either discharged or needed to be released from } \\
\text { the wound } \\
\text { - Follow-up: } 4 \text { weeks } \\
\text { - SSSI N infected/N patients (\%): PG: } 1 / 48(2.1 \%) ; \mathrm{CG}: 2 / 49(4.1 \%) \\
\text { - DSSI not reported } \\
\text { - Adverse effects: reported: "the administration of cephalordine did not result in any change in the type } \\
\text { of organism or curiously in the emergence of cephaloridine-resistant organisms" }\end{array}$ \\
\hline
\end{tabular}


- The study was realised in patients who underwent types of surgery other than hernia repair, but it is possible to extract the information on wound infection related to the patients who underwent hernia repair.

- Antibiotics (Cephaloridine) were supplied by Glaxo laboratories Ltd.

\section{Risk of bias}

\begin{tabular}{|c|c|c|}
\hline Bias & Authors' judgement & Support for judgement \\
\hline $\begin{array}{l}\text { Random sequence genera- } \\
\text { tion (selection bias) }\end{array}$ & Low risk & $\begin{array}{l}\text { Quote: "The method of random selection has been, as before, by the toss of a } \\
\text { coin" }\end{array}$ \\
\hline $\begin{array}{l}\text { Allocation concealment } \\
\text { (selection bias) }\end{array}$ & Unclear risk & Insufficient information available \\
\hline $\begin{array}{l}\text { Blinding of participants } \\
\text { and personnel (perfor- } \\
\text { mance bias) }\end{array}$ & Low risk & $\begin{array}{l}\text { Insufficient information available, but performance bias is unlikely in this set- } \\
\text { ting }\end{array}$ \\
\hline All outcomes & & $\begin{array}{l}\text { Quote: "To avoid bias the details were entered not in the patients' case notes } \\
\text { but on punch cards which were kept separately and analysed manually." }\end{array}$ \\
\hline $\begin{array}{l}\text { Blinding of outcome as- } \\
\text { sessment (detection bias) } \\
\text { All outcomes }\end{array}$ & High risk & Quote: "All patients were seen by one of us for at least 4 weeks." \\
\hline \multirow{2}{*}{$\begin{array}{l}\text { Incomplete outcome data } \\
\text { (attrition bias) } \\
\text { All outcomes }\end{array}$} & High risk & $\begin{array}{l}\text { Patients died from unexplained reasons after surgery and were excluded from } \\
\text { analysis. }\end{array}$ \\
\hline & & $\begin{array}{l}\text { Quote: "Thirty-two patients (out of total } 762 \text { patients) who died within } 2 \text { weeks } \\
\text { of operation in whom the presence or absence of infection was uncertain have } \\
\text { been excluded from the study,..." }\end{array}$ \\
\hline $\begin{array}{l}\text { Selective reporting (re- } \\
\text { porting bias) }\end{array}$ & Low risk & $\begin{array}{l}\text { The study protocol was not registered, but report includes all expected out- } \\
\text { comes, including specification of SSSI }\end{array}$ \\
\hline Other bias & Low risk & The study appears to be free of other sources of bias \\
\hline
\end{tabular}

\section{Goyal 2011}

\section{Study characteristics}

\begin{tabular}{|c|c|}
\hline Methods & $\begin{array}{l}\text { - } \text { RCT } \\
\text { - Enrolment period: } 1 \text { year (not specified) } \\
\text { - Country: India } \\
\text { - Single centre }\end{array}$ \\
\hline Participants & $\begin{array}{l}\text { - } \mathrm{N}: 200 \text { participants } \\
\text { - Age (years): range } 11 \text { to } 90 \text { yrs, with } 70.5 \% \text { of the patients in the } 31 \text { to } 70 \text { years age group (overall } \\
\text { - \% Men: not reported; both male and female were included } \\
\text { - Inclusion criteria: any type of primary inguinal hernia. } \\
\text { - Exclusion criteria: patients with complicated, strangulated hernia, those having local skin infection, } \\
\text { systemic infection, diabetes or history of antibiotic use within previous week } \\
\text { - BMI: not reported } \\
\text { - Skin antiseptic: } 10 \% \text { povidone iodine }\end{array}$ \\
\hline
\end{tabular}


- Operative time: not reported

- Method of repair: Lichtenstein technique (hernioplasty)

- Drain use: not reported

- ASA class: not reported

- Anaesthesia method: not reported

- Hospitalisation: inpatient

- Length of hospital stay: 1 day

- Surgeon: not reported

\begin{tabular}{|c|c|}
\hline Interventions & $\begin{array}{l}\text { - Prophylactic group (PG): } \\
\text { - } \mathrm{N}: 100 \\
\text { - antibiotic: amoxicillin-clavulanate } \\
\text { - dose: } 1.2 \mathrm{~g} \\
\text { - number of doses: } 1 \\
\text { - route of administration: intravenous } \\
\text { - timing of administration: induction } \\
\text { - Control group (CG): } \\
\text { - N: } 100 \\
\text { - substance: equal volume of sterile saline }\end{array}$ \\
\hline Outcomes & $\begin{array}{l}\text { - Wound infection definition: serous discharge, seroma formation, erythema and stitch abscess. } \\
\text { - Follow-up: } 8 \text { days } \\
\text { - SSSI N infected/N patients (\%) PG: } 1 / 100 \text { (1\%); CG: } 3 / 100 \text { (3\%) (SSSI cases had erythema or stitch ab- } \\
\text { scess, cases with serous discharge were considered not having wound infection) } \\
\text { - DSSI: not reported (reported: "not necessary to remove any mesh") } \\
\text { - Adverse effects: not reported }\end{array}$ \\
\hline Notes & $\begin{array}{l}\text { - Patients aged }<18 \text { years included } \\
\text { - Funding was not reported. }\end{array}$ \\
\hline
\end{tabular}

\section{Risk of bias}

\begin{tabular}{|c|c|c|}
\hline Bias & Authors' judgement & Support for judgement \\
\hline $\begin{array}{l}\text { Random sequence genera- } \\
\text { tion (selection bias) }\end{array}$ & Low risk & $\begin{array}{l}\text { Quote: "Patients were randomized in two groups by random number table } \\
\text { method, Group } 1 \text { as cases, Group } 2 \text { as controls" }\end{array}$ \\
\hline $\begin{array}{l}\text { Allocation concealment } \\
\text { (selection bias) }\end{array}$ & Unclear risk & Insufficient information available. \\
\hline $\begin{array}{l}\text { Blinding of participants } \\
\text { and personnel (perfor- } \\
\text { mance bias) }\end{array}$ & Low risk & $\begin{array}{l}\text { Insufficient information available, but performance bias is unlikely in this set- } \\
\text { ting }\end{array}$ \\
\hline All outcomes & & $\begin{array}{l}\text { Quote: "Group } 1 \text { was given IV injection of } 1,2 \text { gr amoxicillin-clavulanate in } 20 \\
\text { ml saline at the time of induction, while the other group was given } 20 \mathrm{ml} \text { of } \\
\text { sterile saline as placebo" }\end{array}$ \\
\hline $\begin{array}{l}\text { Blinding of outcome as- } \\
\text { sessment (detection bias) } \\
\text { All outcomes }\end{array}$ & Unclear risk & No information available \\
\hline $\begin{array}{l}\text { Incomplete outcome data } \\
\text { (attrition bias) } \\
\text { All outcomes }\end{array}$ & Low risk & No missing outcome data \\
\hline
\end{tabular}


Goyal 2011 (Continued)

Selective reporting (reporting bias)

The study protocol was not registered, but report includes all expected outcomes, including specification of SSSI

\begin{tabular}{ll}
\hline Other bias $\quad$ Low risk $\quad$ The study appears to be free of other sources of bias. \\
\hline
\end{tabular}

ljaz 2010

\section{Study characteristics}

\begin{tabular}{|c|c|}
\hline Methods & $\begin{array}{l}\text { - RCT } \\
\text { - Enrolment period: January } 2007 \text { to December } 2007 \\
\text { - Country: Pakistan } \\
\text { - Single centre }\end{array}$ \\
\hline Participants & $\begin{array}{l}\text { - N: } 100 \text { participants } \\
\text { - Age (years; mean) PG: } 44.06 ; \mathrm{CG}: 44.84 \\
\text { - } \% \text { Men: } 99 \% \text { (overall study population) } \\
\text { - Inclusion criteria: elective inguinal hernia, age }>20 \text { years } \\
\text { - Exclusion criteria: patients with obstructed, strangulated or recurrent hernias, patients with immuno- } \\
\text { suppressive disease or on medication (steroids) and patients with a debilitating disease like chronic } \\
\text { liver, renal or cardiac impairment. Also patients allergic to the given antibiotic or taking some antibi- } \\
\text { otic } 7 \text { days prior to surgery for any reason. } \\
\text { - BMl: not reported } \\
\text { - Skin antiseptic: not reported } \\
\text { - Operative time: not reported } \\
\text { - Method of repair: Lichtenstein technique (hernioplasty); polypropylene mesh } \\
\text { - Drain use: not reported } \\
\text { - ASA class: not reported } \\
\text { - Anaesthesia method: spinal } \\
\text { - Hospitalisation method: inpatient } \\
\text { - Length of hospital stay: } 2 \text { days (discharge on second postoperative day) } \\
\text { - Surgeon: senior registrar }\end{array}$ \\
\hline Interventions & $\begin{array}{l}\text { - Prophylactic group (PG): } \\
\text { - } \mathrm{N}: 50 \\
\text { - antibiotic: cefazolin } \\
\text { - dose: } 1 \mathrm{~g} \\
\text { - number of doses: } 1 \\
\text { - route of administration: intravenous } \\
\text { - timing of administration: } 30 \text { minutes before surgery } \\
\text { - Control group (CG): } \\
\text { - } \mathrm{N}: 50 \\
\text { - substance: distilled water }\end{array}$ \\
\hline Outcomes & $\begin{array}{l}\text { - Wound infection definition: CDC criteria } \\
\text { - Follow-up: 7, } 14,30 \text { days post operation } \\
\text { - SSSI N infected/N patients (\%): PG: } 2 / 50(4 \%) ; C G: 5 / 50(10 \%) \\
\text { - DSSI: not reported } \\
\text { - Adverse effects: "None of the patients developed adverse reactions related to antibiotics prophylaxis" }\end{array}$ \\
\hline
\end{tabular}


- Funding was not reported.

\begin{tabular}{|c|c|c|}
\hline \multicolumn{3}{|l|}{ Risk of bias } \\
\hline Bias & Authors' judgement & Support for judgement \\
\hline $\begin{array}{l}\text { Random sequence genera- } \\
\text { tion (selection bias) }\end{array}$ & Unclear risk & $\begin{array}{l}\text { Insufficient information available on how randomisation was performed. } \\
\text { Quote: "All included patients were randomized into two groups A and B and } \\
\text { prepared for surgery". Quote: "Simple random sampling technique was ap- } \\
\text { plied" }\end{array}$ \\
\hline $\begin{array}{l}\text { Allocation concealment } \\
\text { (selection bias) }\end{array}$ & Unclear risk & No information available. \\
\hline $\begin{array}{l}\text { Blinding of participants } \\
\text { and personnel (perfor- } \\
\text { mance bias) } \\
\text { All outcomes }\end{array}$ & Low risk & No information available, but performance bias is unlikely in this setting \\
\hline $\begin{array}{l}\text { Blinding of outcome as- } \\
\text { sessment (detection bias) } \\
\text { All outcomes }\end{array}$ & Unclear risk & No information available. \\
\hline $\begin{array}{l}\text { Incomplete outcome data } \\
\text { (attrition bias) } \\
\text { All outcomes }\end{array}$ & Low risk & No missing outcome data \\
\hline $\begin{array}{l}\text { Selective reporting (re- } \\
\text { porting bias) }\end{array}$ & Low risk & $\begin{array}{l}\text { The study protocol was not registered, but report includes all expected out- } \\
\text { comes, including specification of SSSI }\end{array}$ \\
\hline Other bias & Low risk & The study appears to be free from other sources of bias \\
\hline
\end{tabular}

Jain 2008

\begin{tabular}{|c|c|}
\hline \multicolumn{2}{|c|}{ Study characteristics } \\
\hline Methods & $\begin{array}{l}\text { - Double-blind RCT } \\
\text { - Enrolment period: } 1 \text { year (not specified) } \\
\text { - Country: India } \\
\text { - Single centre }\end{array}$ \\
\hline Participants & $\begin{array}{l}\text { - N: } 120 \text { participants } \\
\text { - Age (years; mean } \pm \text { SD): PG: } 41.28 \pm 11.49 \text {; CG: } 40.2 \pm 9.84 \\
\text { - } \% \text { Men: } 100 \% \text { (both groups) } \\
\text { - Inclusion criteria: primary, unilateral inguinal hernia (direct and indirect) } \\
\text { - Exclusion criteria: recurrent, bilateral, irreducible or strangulated hernia; patients with systemic and } \\
\text { advanced diseases (e.g. liver failure, chronic renal failure, diabetes); immuno-compromised patients; } \\
\text { patients with ASA scores higher than } 11 \text {; patients receiving steroids for any reason; patients younger } \\
\text { than } 18 \text { or older than } 60 ; \text { patients with local skin infections or disease at the site of incision; and pa- } \\
\text { tients allergic to antibiotics or who had received antibiotics less than a week before surgery. } \\
\text { - BMI: not reported } \\
\text { - Skin antiseptic: povidone iodine and alcohol }\end{array}$ \\
\hline
\end{tabular}


- Operative time (minutes, mean \pm SD): PG: $56.33 \pm 11.67$; CG: $60.33 \pm 6.81$

- Method of repair: prolene hernia system (PHS) mesh repair with prolene mesh (hernioplasty).

- Drain use: no drains were used

- ASA class: PG: ASAI $n=52$ (87\%), ASAII $n=8(13 \%)$; CG: ASAI $n=49(82 \%)$, ASAll $n=11(18 \%)$

- Anaesthesia method: local or spinal

- Hospitalisation method: inpatient

- Length of hospital stay: 1 day

- Surgeon: not reported

\begin{tabular}{|c|c|}
\hline Interventions & $\begin{array}{l}\text { - Prophylactic group (PG): } \\
\text { - } \mathrm{N}: 60 \\
\text { - dostibiotic: amoxicillin-clavulanic acid } 1.2 \mathrm{~g} \\
\text { - number of doses: } 1 \\
\text { - route of administration: intravenous } \\
\text { - timing of administration: before incision } \\
\text { - Control group (CG): } \\
\text { - N: } 60 \\
\text { - substance: sterile saline }\end{array}$ \\
\hline Outcomes & $\begin{array}{l}\text { - Wound infection definition: CDC criteria } \\
\text { - Follow-up: till discharge, } 7 \text { to } 9 \text { days, } 2 \text { and } 4 \text { weeks, } 1 \text { year } \\
\text { - SSSI N infected/N patients (\%): PG: } 1 / 60(1.7 \%) \text {; CG: } 1 / 60(1.7 \%) \\
\text { - DSSI N infected/N patients (\%): PG: } 0 / 60(0 \%) ; C G: 0 / 60(0 \%) \\
\text { - Adverse effects: "None of the patients developed adverse reactions related to antibiotics prophylaxis" }\end{array}$ \\
\hline Notes & $\begin{array}{l}\text { - Staphylococcus aureus bacteria were isolated from the wound infections } \\
\text { - Funding was not reported. }\end{array}$ \\
\hline
\end{tabular}

\section{Risk of bias}

\begin{tabular}{|c|c|c|}
\hline Bias & Authors' judgement & Support for judgement \\
\hline $\begin{array}{l}\text { Random sequence genera- } \\
\text { tion (selection bias) }\end{array}$ & Low risk & Quote: "Randomization was performed by a computer-generated code..." \\
\hline $\begin{array}{l}\text { Allocation concealment } \\
\text { (selection bias) }\end{array}$ & Low risk & $\begin{array}{l}\text { Quote: "Randomization was performed by a computer-generated code by a ju- } \\
\text { nior resident who was not involved in the surgery, data compilation or patient } \\
\text { follow-up." }\end{array}$ \\
\hline $\begin{array}{l}\text { Blinding of participants } \\
\text { and personnel (perfor- } \\
\text { mance bias) } \\
\text { All outcomes }\end{array}$ & Low risk & $\begin{array}{l}\text { Quote: "The same resident also prepared the antibiotic or the placebo syringes } \\
\text { containing normal saline." }\end{array}$ \\
\hline $\begin{array}{l}\text { Blinding of outcome as- } \\
\text { sessment (detection bias) } \\
\text { All outcomes }\end{array}$ & Low risk & $\begin{array}{l}\text { Quote: "In order to remove any personal bias, the surgeon who performed the } \\
\text { operation was not allowed to follow up their patient." }\end{array}$ \\
\hline $\begin{array}{l}\text { Incomplete outcome data } \\
\text { (attrition bias) } \\
\text { All outcomes }\end{array}$ & Low risk & No missing outcome data \\
\hline $\begin{array}{l}\text { Selective reporting (re- } \\
\text { porting bias) }\end{array}$ & Low risk & $\begin{array}{l}\text { No study protocol available, but the published report includes all expected } \\
\text { outcomes, including specification of SSSI and DSSI. }\end{array}$ \\
\hline
\end{tabular}


Kochhar 2014

\section{Study characteristics}

\begin{tabular}{|c|c|}
\hline Methods & $\begin{array}{l}\text { - } \text { - Enouble-blind RCT } \\
\text { - Country: India } \\
\text { - Single centre }\end{array}$ \\
\hline Participants & $\begin{array}{l}\text { - N: } 217 \text { participants } \\
\text { - Age (years; mean } \pm \text { SD): PG: } 37.42 \pm 9.9 ; \text { CG: } 37.42 \pm 11.5 \\
\text { - } \% \text { Men: } 95.7 \% \text { (overall study population) } \\
\text { - Inclusion criteria: unilateral or bilateral inguinal hernia, age } 15 \text { to } 70 \text { years } \\
\text { - Exclusion criteria: Patients allergic to the given antibiotic, recurrent hernia, strangulated hernia, preg- } \\
\text { nancy or lactation, an immune compromised state, antibiotic treatment within last } 5 \text { days, existing } \\
\text { indication for antibiotic prophylaxis (i.e. valvular heart disease) } \\
\text { - BMI: not reported } \\
\text { - Skin antiseptic: not reported } \\
\text { - Operative time: not reported } \\
\text { - Method of repair: Lichtenstein technique with monofilament polypropylene mesh (hernioplasty) } \\
\text { - Drain use: not reported } \\
\text { - ASA class: not reported } \\
\text { - Anaesthesia method: not reported } \\
\text { - Hospitalisation method: inpatient } \\
\text { - Length of hospital stay: } 1 \text { day } \\
\text { - Surgeon: not reported }\end{array}$ \\
\hline Interventions & $\begin{array}{l}\text { - Prophylactic group (PG): } \\
* \quad \mathrm{~N}: 109 \\
* \text { antibiotic: amoxicillin clavulanic acid } \\
* \text { dose: } 1.2 \mathrm{~g} \\
\text { * number of doses: } 1 \\
\text { * route of administration: intravenous } \\
\text { * timing of administration: just before operation } \\
\text { - Control group (CG): } \\
\text { - } \mathrm{N}=108 \\
\text { - substance: equal volume of sterile saline }\end{array}$ \\
\hline Outcomes & $\begin{array}{l}\text { - Wound infection definition: ASEPSIS criteria purulent discharge, erythema, wound breakdown) } \\
\text { - Follow-up: } 1 \text { and } 4 \text { weeks } \\
\text { - SSSI N infected/N patients (\%): PG: } 4 / 106 \text { (3.8\%); CG: 5/106 (4.7\%) } \\
\text { - DSSI: not reported (reported: "not necessary to remove any mesh") } \\
\text { - Adverse effects: not reported }\end{array}$ \\
\hline Notes & $\begin{array}{l}\text { - Participants }<18 \text { years old were included in this study } \\
\text { - Staphylococcus aureus, E. coli, Klepsiella pneumoniae bacteria were isolated from the wound infec- } \\
\text { tions } \\
\text { - Funding: nil. }\end{array}$ \\
\hline
\end{tabular}




\begin{tabular}{|c|c|c|}
\hline Bias & Authors' judgement & Support for judgement \\
\hline $\begin{array}{l}\text { Random sequence genera- } \\
\text { tion (selection bias) }\end{array}$ & Low risk & $\begin{array}{l}\text { Quote: "All patients were randomized to either intravenous placebo (control } \\
\text { group) or antibiotic prophylaxis (study group) by block randomization". No in- } \\
\text { formation on how randomisation was accomplished. However, the block ran- } \\
\text { domisation method indicates that the randomisation methodology has been } \\
\text { performed carefully to minimise bias. }\end{array}$ \\
\hline $\begin{array}{l}\text { Allocation concealment } \\
\text { (selection bias) }\end{array}$ & Unclear risk & No information available \\
\hline $\begin{array}{l}\text { Blinding of participants } \\
\text { and personnel (perfor- } \\
\text { mance bias) } \\
\text { All outcomes }\end{array}$ & Low risk & No information available, but performance bias is unlikely in this setting \\
\hline $\begin{array}{l}\text { Blinding of outcome as- } \\
\text { sessment (detection bias) } \\
\text { All outcomes }\end{array}$ & Low risk & $\begin{array}{l}\text { Quote: "Surgeon who was not involved in surgery followed the case after } 1 \text { and } \\
4 \text { weeks post-operatively" }\end{array}$ \\
\hline $\begin{array}{l}\text { Incomplete outcome data } \\
\text { (attrition bias) } \\
\text { All outcomes }\end{array}$ & Low risk & $\begin{array}{l}\text { Missing outcome data balanced in numbers across intervention groups, with } \\
\text { similar reason for missing data. The proportion of missing outcomes was con- } \\
\text { sidered not enough to have had a clinically relevant impact on the interven- } \\
\text { tion effect estimate. } \\
\text { Quote: "Out of the total study population } 3 \text { patients from the study and } 2 \text { pa- } \\
\text { tients from the control group were excluded postoperatively for development } \\
\text { of URI and thus requirement of antibiotics." Available-case analysis was per- } \\
\text { formed. }\end{array}$ \\
\hline $\begin{array}{l}\text { Selective reporting (re- } \\
\text { porting bias) }\end{array}$ & Low risk & $\begin{array}{l}\text { The study protocol was not available but report includes all expected out- } \\
\text { comes, including specification of SSSI }\end{array}$ \\
\hline Other bias & Low risk & The study appears to be free of other sources of bias \\
\hline
\end{tabular}

\section{Lazorthes 1992}

\begin{tabular}{|c|c|}
\hline \multicolumn{2}{|c|}{ Study characteristics } \\
\hline Methods & $\begin{array}{l}\text { - Double-blind RCT } \\
\text { - Enrolment period: October } 1987 \text { to March } 1989 \\
\text { - Country: France } \\
\text { - Single centre }\end{array}$ \\
\hline Participants & $\begin{array}{l}\text { - } \mathrm{N}: 324 \text { participants } \\
\text { - Age (years; mean (range)): PG: } 62 \text { (11 to 90); CG: } 70 \text { (16 to 92) } \\
\text { - \% Men: PG: } 90 \% ; \text { CG: } 88 \% \\
\text { - Inclusion criteria: primary inguinal hernia } \\
\text { - Exclusion criteria: allergy to antibiotics, recurrent hernia, anaesthesia other than local (epidural or } \\
\text { general) } \\
\text { - BMI: not reported } \\
\text { - Skin antiseptic: not reported } \\
\text { - Operative time: not reported }\end{array}$ \\
\hline
\end{tabular}




\begin{tabular}{|c|c|}
\hline dacortiles 1992 (Continuea) & $\begin{array}{l}\text { - Method of repair: not reported (herniorrhaphy) } \\
\text { - Drain use: not reported } \\
\text { - ASA class: not reported } \\
\text { - Anaesthesia method: local } \\
\text { - Hospitalisation method: inpatient } \\
\text { - Length of hospital stay: not specified, hospital stay was prolonged due to infection } \\
\text { - Surgeon: not reported }\end{array}$ \\
\hline Interventions & $\begin{array}{l}\text { - Prophylactic group (PG): } \\
\text { - } \mathrm{N}: 162 \\
\text { - antibiotic: cefamandole } \\
\text { - dose: } 750 \mathrm{mg} \\
\text { - number of doses: } 1 \\
\text { - route of administration: added to local anaesthesia solution (lidocaine) } \\
\text { - timing of administration: during anaesthesia/ surgery } \\
\text { - Control group (CG): } \\
\text { - N: } 162 \\
\text { - substance: } \text { no antibiotics, only local anaesthesia (lidocaine) solution }\end{array}$ \\
\hline Outcomes & $\begin{array}{l}\text { - Wound infection definition: wounds with discharge with positive microbiological culture } \\
\text { - Follow-up: till } 1 \text { month } \\
\text { - SSSI N infected/N patients (\%): PG: 0/155 (0\%)/ CG: } 7 / 153(4.6 \%) \\
\text { - DSSI: not reported } \\
\text { - Adverse effects: "No instances of allergy" }\end{array}$ \\
\hline Notes & $\begin{array}{l}\text { - Participants }<18 \text { years old were included in this study } \\
\text { - Staphylococcus aureus, S. epidermidis, S. albans, Streptococcus sanguis, Streptococcus alpha and Es- } \\
\text { cherichia coli bacteria were cultured from the wound infections } \\
\text { - Funding is not reported. }\end{array}$ \\
\hline
\end{tabular}

\section{Risk of bias}

\begin{tabular}{|c|c|c|}
\hline Bias & Authors' judgement & Support for judgement \\
\hline \multirow{2}{*}{$\begin{array}{l}\text { Random sequence genera- } \\
\text { tion (selection bias) }\end{array}$} & Unclear risk & Insufficient information available \\
\hline & & Quote: "Two groups of 162 patients were randomly allotted to receive ..." \\
\hline $\begin{array}{l}\text { Allocation concealment } \\
\text { (selection bias) }\end{array}$ & Unclear risk & No information available \\
\hline $\begin{array}{l}\text { Blinding of participants } \\
\text { and personnel (perfor- } \\
\text { mance bias) } \\
\text { All outcomes }\end{array}$ & Low risk & $\begin{array}{l}\text { Insufficient information available, but performance bias is unlikely in this set- } \\
\text { ting }\end{array}$ \\
\hline
\end{tabular}

Blinding of outcome assessment (detection bias) All outcomes
Unclear risk

Q

Insufficient information available.

Quote: "A questionnaire was given to the patient upon discharge and the patient was asked to return it one month postoperatively to ensure that there was no late wound abscess. In the instance of wound discharge, the patient was examined by the surgeon to ascertain its true nature."

\footnotetext{
Incomplete outcome data Low risk (attrition bias)

All outcomes

Missing outcome data are balanced in numbers across intervention groups (PG $n=7(4.3 \%) ; C G n=9(5.5 \%))$. The proportion of missing outcomes was consid-
} 
ered not enough to have had a clinically relevant impact on the intervention effect estimate

Available case analysis was performed.

\begin{tabular}{ll}
\hline $\begin{array}{l}\text { Selective reporting (re- } \\
\text { porting bias) }\end{array}$ & Low risk \\
outcomes, including specification of SSSI.
\end{tabular}

\begin{tabular}{ll}
\hline Other bias $\quad$ Low risk $\quad$ The study appears to be free of other sources of bias \\
\hline
\end{tabular}

Mazaki 2013

\section{Study characteristics}

\begin{tabular}{|c|c|}
\hline Methods & $\begin{array}{l}\text { - Double-blind RCT } \\
\text { - Enrolment period: July } 2007 \text { to December } 2011 \\
\text { - Country: Japan } \\
\text { - Single centre }\end{array}$ \\
\hline Participants & 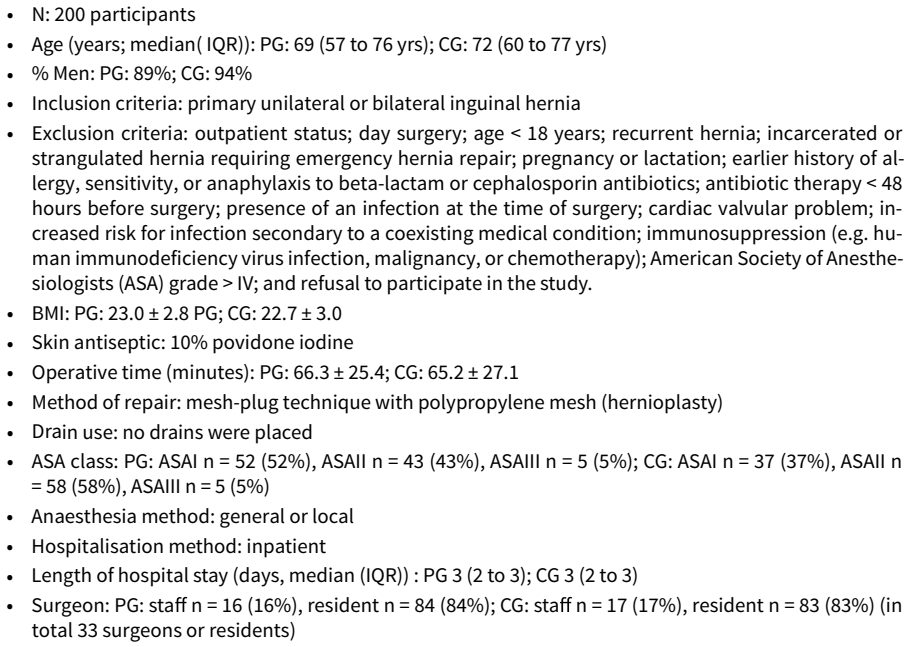 \\
\hline Interventions & $\begin{array}{l}\text { - Prophylactic group (PG): } \\
\text { - } \mathrm{N}: 100 \\
\text { - antibiotic: cefazolin } \\
\text { - dose: } 1 \mathrm{~g} \\
\text { - number of doses: } 1 \\
\text { - route of administration: intravenous (CG: continuous IV, PG: single administration) } \\
\text { - timing of administration: } 30 \text { minutes before incision } \\
\text { - Control group (CG): }\end{array}$ \\
\hline
\end{tabular}


- substance: equal volume sterile saline

\begin{tabular}{ll}
\hline Outcomes & Wound infection definition: CDC criteria \\
& - Follow-up: 7 to 8 days, 1 month and 3 months post operation \\
& - SSSI N infected/N patients (\%): PG: $2 / 100(2 \%) ; C G: 13 / 100(13 \%)$ \\
& DSSI: N infected/N patients (\%): PG: $0 / 100(0 \%) ; C G: 0 / 100(0 \%)$ \\
\hline Notes & Adverse effects: "No adverse events were seen nor mortality" \\
\hline
\end{tabular}

\section{Risk of bias}

\begin{tabular}{|c|c|c|}
\hline Bias & Authors' judgement & Support for judgement \\
\hline $\begin{array}{l}\text { Random sequence genera- } \\
\text { tion (selection bias) }\end{array}$ & Low risk & Quote: “.....computer-generated list in blocks of 50 patients." \\
\hline $\begin{array}{l}\text { Allocation concealment } \\
\text { (selection bias) }\end{array}$ & Low risk & $\begin{array}{l}\text { Quote: “.... a pharmacist carried out randomization" } \\
\text { Quote: "All surgeons and other staff members were blinded to randomization } \\
\text { and to patients' details" }\end{array}$ \\
\hline $\begin{array}{l}\text { Blinding of participants } \\
\text { and personnel (perfor- } \\
\text { mance bias) } \\
\text { All outcomes }\end{array}$ & Low risk & $\begin{array}{l}\text { Quote: "All surgeons and other staff members were blinded to randomization } \\
\text { and to patients". } \\
\text { Quote: "Patients were randomly assigned on admission in a double-blinded } \\
\text { manner to either the antibiotic prophylaxis group or the placebo group." }\end{array}$ \\
\hline $\begin{array}{l}\text { Blinding of outcome as- } \\
\text { sessment (detection bias) } \\
\text { All outcomes }\end{array}$ & Low risk & $\begin{array}{l}\text { Quote: "All wounds were carefully examined by } 2 \text { certified surgeons, who did } \\
\text { not perform the operations" }\end{array}$ \\
\hline $\begin{array}{l}\text { Incomplete outcome data } \\
\text { (attrition bias) } \\
\text { All outcomes }\end{array}$ & Low risk & $\begin{array}{l}\text { Flow chart of participants reveals missing outcome data is low ( } \mathrm{n}=1 \text { in antibi- } \\
\text { otic prophylaxis group and } \mathrm{n}=3 \text { in placebo group), with similar reasons for } \\
\text { missing data (other surgical technique applied) } \\
\text { Also intention-to-treat analysis was applied. }\end{array}$ \\
\hline $\begin{array}{l}\text { Selective reporting (re- } \\
\text { porting bias) }\end{array}$ & Low risk & $\begin{array}{l}\text { The protocol was registered (NCT00636831) and study outcomes that are of in- } \\
\text { terest in the review have been reported in the prespecified way. }\end{array}$ \\
\hline Other bias & High risk & $\begin{array}{l}\text { Study was terminated early after interim analysis, and therefore there might } \\
\text { be risk of bias for increased benefit in the antibiotics prophylaxis group. }\end{array}$ \\
\hline
\end{tabular}

Morales 2000

\section{Study characteristics}

\begin{tabular}{ll}
\hline Methods & Double-blind RCT \\
& Enrolment period: June 1994 to June 1997 \\
& - Country: Spain/lbiza \\
& Multicentre (3) \\
\hline Participants & N: 554 participants
\end{tabular}


- Age (years; mean (range)): 54.2 (17 to $87 y r s)$ (overall study population)

- \% Men: $89.9 \%$ (overall study population)

- Inclusion criteria: inguinal or femoral hernia repair with synthetic mesh

- Exclusion criteria: emergency surgery, cytostatic or corticosteroid therapy, HIV/AIDS or neoplastic disease, non-compliance of the allocation concealment, no fulfilment of the time of follow-up according to the protocol, drainage placement.

- BMI: not reported

- Skin antiseptic: $10 \%$ povidone iodine

- Operative time: not reported (in patients with infections the mean operation time was 34 minutes and in patients without infections the mean operation time was 36.5 minutes)

- Method of repair: polypropylene mesh repair (hernioplasty)

- Drain use: patients with drains were excluded

- ASA class: ASAI $n=224$ (42.7\%), ASAII $n=219$ (41.8\%), ASAIII $n=78$ (14.9\%) (overall study population, not specified per treatment group)

- Anaesthesia method: not reported

- Hospitalisation method: inpatient and outpatient

- Length of hospital stay: 0 to 1 day

- Surgeon: only staff, no residents

\begin{tabular}{ll}
\hline Interventions & Prophylactic group (PG): \\
& - $\mathrm{N}: 237$ \\
& - antibiotic: cefazolin or Erythromycin (in case of allergy to cefazolin) \\
& - dose: $2 \mathrm{~g}$ ( $1 \mathrm{~g}$ for erythromycin) \\
& - number of doses: 1 \\
& - route of administration: intravenous \\
& - timing of administration: at induction \\
& Control group (CG): \\
& - N: 287 \\
& - substance: physiological serum
\end{tabular}

Outcomes

- Wound infection definition: presence of 1 of the following criteria: 1 ) skin erythema $>2 \mathrm{~cm}$ on both sides of the incision; 2) discharge of purulent material from the wound; 3 ) discharge not purulent with microbiological positive culture; 4) wound had to be re-opened by the surgeon

- Follow-up: 7 days, 1 month, 12 months post operation.

- SSSI N infected/N patients (\%): PG: 4/233 (1.6\%); CG: 6/281 (2.1\%)

- DSSI N infected/N patients (\%): 4 patients (all with previous SSI, not specified in which treatment group)

- Adverse effects: not reported

\begin{tabular}{ll}
\hline Notes & - S. aureus, E. Coli + Proteus mirabilis were isolated from the wound infections \\
& - Spanish manuscript \\
- Funding was not reported.
\end{tabular}

\section{Risk of bias}

\begin{tabular}{lll}
\hline Bias & Authors' judgement & Support for judgement \\
\hline $\begin{array}{l}\text { Random sequence genera- } \\
\text { tion (selection bias) }\end{array}$ & Low risk & Marked cards \\
& $\begin{array}{l}\text { Quote: "una classificaccion de los pacientos de forma aleatona mediante la } \\
\text { extraccion de tarjetas marcades como "SF" (profilaxis) o "no" (placebo) (= a } \\
\text { classification of the patients in a random way by extracting cards marked as } \\
\text { "SF" (prophylaxis) or "no" (placebo))" }\end{array}$ \\
\hline
\end{tabular}


Quote: "se designo un coordinator del estudio en cada centro respondable de la recigida de datos y de velar por el cumplimiento de los criterios de inclusion de los pacientes, asi como de la aleatorizacion, estraficacion y aleatoriedad del estudio, y de la confidencialidad de los resultados (= a study coordinator was designated in each center responsible for the data collection and to ensure compliance with the inclusion criteria of the patients, as well as the randomization, and the confidentiality of the results)"

\begin{tabular}{ll}
\hline $\begin{array}{l}\text { Blinding of participants } \\
\text { and personnel (perfor- }\end{array}$ & Quote: "La administración de la solución (antibiótico o placebo) fue realiza- \\
mance bias) & $\begin{array}{l}\text { da por la enfermera circulante, no informando del contenido de la solución a } \\
\text { ninguno de los dos cirujanos implicados en la intervención." (= "The adminis- } \\
\text { All outcomes }\end{array}$ \\
& $\begin{array}{l}\text { tration of the solution (antibiotic or placebo) was performed by the nurse, not } \\
\text { informing the content of the solution to any of the two surgeons involved in } \\
\text { the intervention.") }\end{array}$
\end{tabular}

\begin{tabular}{|c|c|c|}
\hline $\begin{array}{l}\text { Blinding of outcome as- } \\
\text { sessment (detection bias) } \\
\text { All outcomes }\end{array}$ & Low risk & $\begin{array}{l}\text { Quote: "En el seguimiento postoperatorio, los cirujanos, las enfermeras de } \\
\text { planta y la de consulta externa desconocían la solución administrada al pa- } \\
\text { ciente." and "control en la consulta externa de cirurgia (= "In the postopera- } \\
\text { tive follow-up, the surgeons, the plant nurses and the outpatient nurses were } \\
\text { unaware of the solution administered to the patient" and "control in the out- } \\
\text { patient clinic of surgery") }\end{array}$ \\
\hline
\end{tabular}

\begin{tabular}{lll}
\hline $\begin{array}{l}\text { Incomplete outcome data } \\
\text { (attrition bias) } \\
\text { All outcomes }\end{array}$ & Low risk & $\begin{array}{l}\text { In the antibiotic prophylaxis group 4 patients were lost to follow-up and in the } \\
\text { control group 6 patients were lost to follow-up. Missing data is balanced in } \\
\text { numbers across intervention groups. Also the proportion of missing outcomes } \\
\text { compared with the observed event risk is not enough to have had a clinically } \\
\text { relevant impact on the intervention effect estimate. } \\
\text { Available case analysis of the data was performed. }\end{array}$ \\
\hline $\begin{array}{l}\text { Selective reporting (re- } \\
\text { porting bias) }\end{array}$ & Unclear risk & $\begin{array}{l}\text { No reporting that study protocol was registered. Authors failed to report num- } \\
\text { ber of cases with DSSI and mesh removal surgery per treatment group and } \\
\text { therefore meta-analysis for this outcome is not possible. }\end{array}$ \\
\hline Other bias & Low risk & \begin{tabular}{l} 
The study appears to be free of other sources of bias \\
\hline
\end{tabular}
\end{tabular}

\section{Oteiza 2004}

\begin{tabular}{|c|c|}
\hline \multicolumn{2}{|c|}{ Study characteristics } \\
\hline Methods & $\begin{array}{l}\text { - RCT } \\
\text { - Country: Spain } \\
\text { - Enrolment period: March } 2001 \text { to March } 2002 \\
\text { - Single centre }\end{array}$ \\
\hline Participants & $\begin{array}{l}\text { - N: } 250 \text { participants } \\
\text { - Age (years; mean (range)): PG: } 58 \text { (22 to } 91 \text { ); CG: } 56.2 \text { (17 to } 88 \text { ) } \\
\text { - } \% \text { Men: PG: } 89 \% \text {; CG: } 82 \% \\
\text { - Inclusion criteria: unilateral direct and indirect inguinal hernia } \\
\text { - Exclusion criteria: recurrent or bilateral hernia, allergy to penicillin, ASA IV and those who needed } \\
\text { hospitalisation. } \\
\text { - BMI: not reported } \\
\text { - Skin antiseptic: not reported } \\
\text { - Operative time: (mean) } 40 \text { min in both groups } \\
\text { - Method of repair: tension free (Lichtenstein, or Plug-Stein) with polypropylene mesh (hernioplasty) }\end{array}$ \\
\hline
\end{tabular}


- Drain use: not reported

- ASA class: PG: ASAI $n=30$ (24\%), ASAII $n=72$ (58\%), ASAIII $n=22$ (18\%); CG: ASAI $n=35$ (28\%), ASAII $n=70(57 \%)$, ASAIII $n=18(15 \%)$

- Anaesthesia: local with sedation or spinal

- Hospitalisation: outpatient

- Length of hospital stay: no admission

- Surgeon: 2 surgeons

\begin{tabular}{ll}
\hline Interventions & Prophylactic group (PG): \\
& - N: 125 \\
& - antibiotic: amoxicillin-clavulanate \\
& - dose: $2 \mathrm{~g}$ \\
& - number of doses: 1 \\
& - route of administration: intravenous \\
& - timing of administration: 15 to 30 minutes before incision \\
& Control group $(C G):$ \\
& - N: 125 \\
& - substance: nothing
\end{tabular}

\begin{tabular}{ll}
\hline Outcomes & Wound infection definition: discharge of purulent material for the wound, discharge of purulent mate- \\
& rial for the wound, the surgeon felt that would need to be open the wound to drain a possible infection. \\
- Follow-up: 1 week till 1 month post operation \\
- SSSI N infected/N patients $(\%):$ PG: $1 / 124(0.8 \%) ; C G: 0 / 123(0 \%)$ \\
- DSSI: not reported \\
- Adverse effects: not reported \\
\hline Notes \\
- Spanish manuscript \\
- S. epidermidis bacteria were cultured from the wound infection \\
- Funding is not reported.
\end{tabular}

\section{Risk of bias}

\begin{tabular}{|c|c|c|}
\hline Bias & Authors' judgement & Support for judgement \\
\hline $\begin{array}{l}\text { Random sequence genera- } \\
\text { tion (selection bias) }\end{array}$ & Low risk & $\begin{array}{l}\text { Quote:".... mediante una tabla de numeros Aleatorios generade por orde- } \\
\text { nador" (= "using a table of numbers randomly generated by computer ") }\end{array}$ \\
\hline $\begin{array}{l}\text { Allocation concealment } \\
\text { (selection bias) }\end{array}$ & Unclear risk & No information available \\
\hline $\begin{array}{l}\text { Blinding of participants } \\
\text { and personnel (perfor- } \\
\text { mance bias) } \\
\text { All outcomes }\end{array}$ & Low risk & No information available, but performance bias is unlikely in this setting \\
\hline $\begin{array}{l}\text { Blinding of outcome as- } \\
\text { sessment (detection bias) } \\
\text { All outcomes }\end{array}$ & Low risk & $\begin{array}{l}\text { Patient self-reporting. } \\
\text { Quote: "antes de la intervencion los patientes fueron advisadosde la necesi- } \\
\text { dad de acudir a la consulta si tenian fiebre (temperature mayor de } 38 \text { degrees } \\
\text { Celcius), tumefaccion local and/or supuracion de la herida.(= Before the in- } \\
\text { tervention the patients were advised of the need to go to the clinic if they had } \\
\text { a fever (temperature greater than } 38 \text { degrees Celcius), local swelling and / or } \\
\text { suppuration of the wound.)" }\end{array}$ \\
\hline
\end{tabular}


Incomplete outcome data Low risk (attrition bias)

All outcomes

Data analysed as "available case analysis" ( $P G n=1 ; C G n=2)$. Missing outcome data balanced in numbers across intervention groups. Also the proportion of missing outcomes compared with the observed event risk is not enough to have had a clinically relevant impact on the intervention effect estimate.

Quote: "tres pacientes precisaron ingreso despues la cirurgia y fueron excluidos (= "three patients required admission after the surgery and were excluded")

\begin{tabular}{|c|c|c|}
\hline $\begin{array}{l}\text { Selective reporting (re- } \\
\text { porting bias) }\end{array}$ & Low risk & $\begin{array}{l}\text { No study protocol available, but the published report includes all expected } \\
\text { outcomes, including specification of SSSI }\end{array}$ \\
\hline
\end{tabular}

Other bias Low risk Study seems to be free of other sources of bias

Othman 2011

\section{Study characteristics}

\begin{tabular}{|c|c|}
\hline Methods & $\begin{array}{l}\text { - Double-blind RCT } \\
\text { - Country: Saudi Arabia } \\
\text { - Enrolment period: July } 2006 \text { to April } 2010 \\
\text { - Single centre }\end{array}$ \\
\hline Participants & $\begin{array}{l}\text { - N: } 98 \text { participants } \\
\text { - Age (years; mean } \pm \text { SD): PG: } 43.4 \pm 19.8 ; C G: 44.5 \pm 20.5 \\
\text { - } \% \text { Men: PG } 96 \% ; C G 100 \% \\
\text { - Inclusion criteria: unilateral or bilateral inguinal hernia } \\
\text { - Exclusion criteria: strangulated or obstructed hernia, patients on steroid treatment or chemothera- } \\
\text { py or those who received antibiotics in the past } 48 \text { hours and patients with valvular heart disease or } \\
\text { prosthetic heart valves } \\
\text { - BMI: not reported } \\
\text { - Skin antiseptic: } 10 \% \text { povidone iodine } \\
\text { - Operative time: PG } 38.8 \pm 10.8 ; \text { CG } 40.9 \pm 11.1 \\
\text { - Method of repair: Lichtenstein repair with polypropylene mesh (hernioplasty) } \\
\text { - Drain use: not reported } \\
\text { - ASA class: not reported } \\
\text { - Anaesthesia method: general or spinal } \\
\text { - Hospitalisation method: inpatient } \\
\text { - Length of hospital stay (days, mean } \pm \text { SD): PG: } 1.3 \pm 0.463 ; \text { CG: } 1.25 \pm 0.438 \\
\text { - Surgeon: not reported }\end{array}$ \\
\hline Interventions & $\begin{array}{l}\text { - Prophylactic group (PG): } \\
\text { - } \mathrm{N}: 50 \\
\text { - antibiotic: amoxicillin-clavulanic (Augmentin) } \\
\text { - dose: } 1.2 \mathrm{~g} \\
\text { - number of doses: } 1 \\
\text { - route of administration: intravenous } \\
\text { - timing of administration: } 30 \text { minutes before induction } \\
\text { - Control group (CG): } \\
\text { - } \mathrm{N}: 48 \\
\text { - substance: equal volume of sterile saline }\end{array}$ \\
\hline
\end{tabular}




$\begin{array}{ll}\text { - Wutcomes } & \text { Wound infection definition: CDC and APIC criteria } \\ & \text { - Follow-up: follow-up every other day till removal of staples up to } 1 \text { month post operation } \\ \text { - } & \text { SSSI N infected/N patients (\%): PG: } 3 / 50(6 \%) ; C G: 5 / 48(10.4 \%) \\ \text { - } & \text { DSSI N infected/N patients (\%): PG: } 1 / 50(2 \%) ; C G: 1 / 48(2.1 \%) \\ \text { - Adverse effects: not reported }\end{array}$

\section{Risk of bias}

\begin{tabular}{|c|c|c|}
\hline Bias & Authors' judgement & Support for judgement \\
\hline $\begin{array}{l}\text { Random sequence genera- } \\
\text { tion (selection bias) }\end{array}$ & Low risk & $\begin{array}{l}\text { Quote: "Patients were randomly divided into two groups using a computer } \\
\text { randomization program" }\end{array}$ \\
\hline $\begin{array}{l}\text { Allocation concealment } \\
\text { (selection bias) }\end{array}$ & Low risk & $\begin{array}{l}\text { Quote: "Randomization and preparation of drug and placebo were controlled } \\
\text { by a surgery clinic nurse without the previous knowledge of the patient or sur- } \\
\text { geon." }\end{array}$ \\
\hline $\begin{array}{l}\text { Blinding of participants } \\
\text { and personnel (perfor- } \\
\text { mance bias) } \\
\text { All outcomes }\end{array}$ & Low risk & $\begin{array}{l}\text { Quote: "Randomization and preparation of drug and placebo were controlled } \\
\text { by a surgery clinic nurse without the previous knowledge of the patient or sur- } \\
\text { geon." }\end{array}$ \\
\hline $\begin{array}{l}\text { Blinding of outcome as- } \\
\text { sessment (detection bias) } \\
\text { All outcomes }\end{array}$ & Unclear risk & Insufficient information available \\
\hline $\begin{array}{l}\text { Incomplete outcome data } \\
\text { (attrition bias) } \\
\text { All outcomes }\end{array}$ & Low risk & No missing outcome data \\
\hline $\begin{array}{l}\text { Selective reporting (re- } \\
\text { porting bias) }\end{array}$ & Low risk & $\begin{array}{l}\text { The study protocol is not available but report includes all expected outcomes, } \\
\text { including specification of SSSI and DSSI }\end{array}$ \\
\hline Other bias & Low risk & The study appears to be free of other sources of bias \\
\hline
\end{tabular}

\section{Perez 2005}

\section{Study characteristics}

\begin{tabular}{ll}
\hline Methods & Double-blind RCT \\
- & Country: Republic of the Philippines \\
- & Enrolment period: January 2000 to December 2002 \\
- & single centre \\
\hline Participants & $\mathrm{N}: 360$ participants \\
- Age (years; mean $\pm \mathrm{SD}$ ): PG: $61.37 \pm 13.2 ; \mathrm{CG}: 60.8 \pm 14.5$ \\
- $\%$ Men: $98 \%$ (both groups) \\
- Exclusion criteria: primary unilateral (direct and indirect) inguinal hernia. \\
\\
anaphylaxis to beta-lactam or cephalosporin antibiotics; antibiotic therapy within 48 hours before
\end{tabular}


operation; presence of infection at the time of operation; patients with cardiac valvular problems; patients with prosthetic valves or joints; patients determined to be at increased risk of infection secondary to a coexisting medical condition; and patients with ASA class more than II.

- BMI: not reported

- Skin antiseptic: povidone iodine

- Operative time (minutes) (mean \pm SD): PG 52.18 \pm 16.4 ; CG $54.07 \pm 15.3$

- Method of repair: Lichtenstein technique with polypropylene mesh (hernioplasty)

- Drain use: no drains were used

- ASA class: PG ASAI $n=146$ (81\%); ASAII $n=34$ (19\%); CG ASAI $n=138(77 \%)$; ASAII $n=42(23 \%)$

- Anaesthesia method: regional

- Hospitalisation method: not reported

- Length of hospital stay: not reported

- Surgeon: senior surgical residents or consultants

\begin{tabular}{|c|c|}
\hline Interventions & $\begin{array}{l}\text { Prophylactic group (PG): } \\
\text { - } \mathrm{N}: 180 \\
\text { - antibiotic: cefazolin } \\
\text { - nume: } 1 \mathrm{~g} \\
\text { - route of administration: intravenous } \\
\text { - timing of administration: before incision } \\
\text { - Control group (CG): } \\
\text { - } \mathrm{N}: 180 \\
\text { - substance: equal volume of sterile saline }\end{array}$ \\
\hline Outcomes & $\begin{array}{l}\text { - Wound infection definition: CDC criteria } \\
\text { - Follow-up: at discharge, } 7 \text { days post operation, } 2 \text { and } 4 \text { weeks post discharge } \\
\text { - SSSI N infected/N patients (\%): PG: } 3 / 180(1.7 \%) ; C G: 6 / 180(3.4 \%) \\
\text { - DSSI N infected/N patients (\%): PG: } 1 / 180(0.6 \%) ; \text { CG: } 1 / 180(0.6 \%) \\
\text { - Adverse effects: not reported }\end{array}$ \\
\hline Notes & $\begin{array}{l}\text { - S. aureus, S. epidermidis were cultured from SSSI wound infections and S. aureus and Pseudomonas } \\
s p \text { bacteria were cultured from infected meshes } \\
\text { - Reported: no competing interest }\end{array}$ \\
\hline
\end{tabular}

\section{Risk of bias}

\begin{tabular}{|c|c|c|}
\hline Bias & Authors' judgement & Support for judgement \\
\hline $\begin{array}{l}\text { Random sequence genera- } \\
\text { tion (selection bias) }\end{array}$ & Low risk & $\begin{array}{l}\text { Quote: "Allocation was done with simple randomization using a comput- } \\
\text { er-generated table of random numbers." }\end{array}$ \\
\hline $\begin{array}{l}\text { Allocation concealment } \\
\text { (selection bias) }\end{array}$ & Low risk & $\begin{array}{l}\text { Quote: "Randomization and preparation of drug and placebo were controlled } \\
\text { by a surgery clinic nurse without the previous knowledge of the patient or sur- } \\
\text { geon." }\end{array}$ \\
\hline $\begin{array}{l}\text { Blinding of participants } \\
\text { and personnel (perfor- } \\
\text { mance bias) } \\
\text { All outcomes }\end{array}$ & Low risk & $\begin{array}{l}\text { Quote: "Senior surgical residents or consultants, blinded to the study group, } \\
\text { performed all operations." }\end{array}$ \\
\hline $\begin{array}{l}\text { Blinding of outcome as- } \\
\text { sessment (detection bias) } \\
\text { All outcomes }\end{array}$ & Low risk & $\begin{array}{l}\text { Quote: "All wounds were inspected before discharge, and all incisions were } \\
\text { carefully reexamined by an independent surgeon blinded to the study during } \\
\text { first followup visit..." }\end{array}$ \\
\hline
\end{tabular}




\begin{tabular}{lll}
\hline $\begin{array}{l}\text { Selective reporting (re- } \\
\text { porting bias) }\end{array}$ & Low risk & $\begin{array}{l}\text { The study protocol is not available but report includes all expected outcomes, } \\
\text { including specification of SSSI and DSSI }\end{array}$ \\
\hline Other bias & Low risk & The study appears to be free of other sources of bias \\
\hline
\end{tabular}

\section{Platt 1990}

\section{Study characteristics}

\begin{tabular}{|c|c|}
\hline Methods & $\begin{array}{l}\text { - Double-blind RCT } \\
\text { - Country: USA } \\
\text { - Enrolment period: } 1 \text { April } 1985 \text { to } 30 \text { September } 1987 \\
\text { - Multicentre (6) }\end{array}$ \\
\hline Participants & 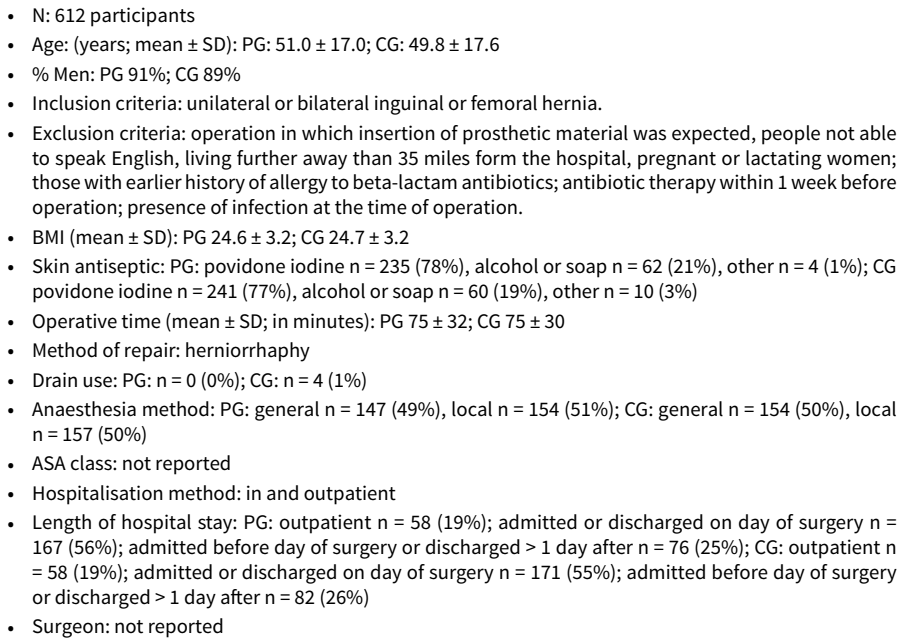 \\
\hline
\end{tabular}

Interventions

- Prophylactic group (PG):

- N: 301

- antibiotic: cefonicid

- dose: $1 \mathrm{~g}$

- number of doses: 1

- route of administration: intravenous

- timing of administration: half hour before intervention ( $\max 90$ minutes before surgery)

- Control group (CG): 
- substance: $50 \mathrm{ml}$ mixture of glycine, mannitol and riboflavin in $5 \%$ dextrose in water

Outcomes

- Wound infection definition: erythema and drainage, or purulent discharge or a wound that was opened and not reclosed

- Follow-up: to 1 week, 4 to 6 weeks post operation

- SSSI + DSSI N infected/N patients (\%): PG: 4/301 (1.3\%); CG: 6/311 (1.9\%)

- Adverse effects: "no serious adverse events were attributable to treatment with Cefonid or placebo"

- In this study were included patients with other types of surgery (mastectomy, lumpectomy, excisional breast biopsy, axillary-node dissection for breast cancer or reduction mammoplasty. For meta-analysis, only patients who underwent hernia repair were included.

- S. aureus bacteria were cultured from infected wounds.

- Authors (Wikler, Moonsammy, Jarosz) are employees of and stockholders in SmithKline Beecham Ltd.

\section{Risk of bias}

\begin{tabular}{lll}
\hline Bias & Authors' judgement & Support for judgement \\
\hline $\begin{array}{l}\text { Random sequence genera- } \\
\text { tion (selection bias) }\end{array}$ & Low risk & $\begin{array}{l}\text { Quote: "patients....were randomly assigned separately in blocks of } 10 \text { to re- } \\
\text { ceive cefonicid or placebo" }\end{array}$ \\
& $\begin{array}{l}\text { No information on how randomisation was accomplished. However, the block } \\
\text { randomisation method indicates that the randomisation methodology has } \\
\text { been performed carefully to minimise bias. }\end{array}$
\end{tabular}

Allocation concealment Unclear risk (selection bias)
Quote: "the treatment codes were not known by anyone at the participating centers, unless the sealed, opaque label attached to each vial was opened. "

Not specified whether envelopes were sequentially numbered.

Blinding of participants Low risk
and personnel (perfor-
mance bias)
All outcomes

\begin{abstract}
Quote: “"the treatment codes were not known by anyone at the participating centers, unless the sealed, opaque label attached to each vial was opened. .......Investigators were required to return these labels intact or to indicate the reason for opening them."
\end{abstract}

Quote: "none of the personnel at the data processing or coordinating center knew the treatment codes, and the codes were not revealed to ...medical personnel..."

Quote: “...the codes (i.e. treatment codes) were not revealed to the patients..”
Blinding of outcome as- Low risk sessment (detection bias) All outcomes
Quote: “...drug assignments were not known during any follow-up evaluations...for suspected wound infections"
Incomplete outcome data High risk (attrition bias)

All outcomes
Missing outcome data (exclusion of patients from analysis after randomisation) were reported and are balanced across treatment (antibiotic prophylaxis) and placebo group.

However, numbers of missing outcome data are high (PG $n=50$ and CG $n=$ 51 from $n=1319$ randomised patients for all different types of surgical procedures analysed in this study). These data are excluded from data-analysis and available-case analysis was performed. The proportion of missing outcomes compared with the observed event risk is enough to have induced clinically relevant bias in the intervention effect estimate.

The study protocol was not available but report includes expected outcome

wound infection.
Selective reporting (re- Low risk porting bias) 
Rahmani 2012

\section{Study characteristics}

\begin{tabular}{|c|c|}
\hline Methods & $\begin{array}{l}\text { - Double-blind RCT } \\
\text { - Country: Iran } \\
\text { - Enrolment period: } 2010 \text { to } 2011 \\
\text { - Multicentre (2) }\end{array}$ \\
\hline Participants & $\begin{array}{l}\text { - N: } 282 \text { participants } \\
\text { - } \text { groups } 25 \text { to } 84 \text { years old (not specified per treatment group; insignificant difference between treatment } \\
\text { - } \% \text { Men: PG } 44.7 \% \text {; CG } 40.8 \% \\
\text { - Inclusion criteria: primary unilateral inguinal hernias. } \\
\text { - Exclusion criteria: emergency repair of hernias (incarcerated-strangulated hernia), allergy to cefalotin } \\
\text { or penicillin, recurrent hernia, taking antibiotics because of other situations like infectious disease } \\
\text { or cardiac valve disease, patients with deficiency of immune system because of disease (diabetes, } \\
\text { HIV, malignancies) or taking medications (immunosuppressant, corticosteroids), not receiving patient } \\
\text { consent for joining the study } \\
\text { - BMI: not specified per treatment group; insignificant difference between treatment groups (P=0.66) } \\
\text { - Skin antiseptic: not reported } \\
\text { - Operative time: not specified per treatment group, insignificant difference between treatment groups } \\
\text { (P=0.12) } \\
\text { - Method of repair: Lichtenstein technique with polypropylene mesh (hernioplasty) } \\
\text { - Drain use: } \text { PG: } n=11 ; \text { CG } n=7 \\
\text { - ASA class: not reported } \\
\text { - Hospithesia method: not reported } \\
\text { - Length of hospital stay: } \text { not reported } \\
\text { - Surgeon: (Senior) surgeon or resident }\end{array}$ \\
\hline Interventions & $\begin{array}{l}\text { - Prophylactic group (PG): } \\
\text { - } \mathrm{N}: 141 \\
\text { - antibiotic: cephalotin ((keflin) } \\
\text { - dose: } 1 \mathrm{~g} \\
\text { - number of doses: } 1 \\
\text { - route of administration: intravenous } \\
\text { - timing of administration: } 30 \text { minutes before incision } \\
\text { - Control group (CG): } \\
\text { - N: } 141 \\
\text { - substance: equal volume of distilled water }\end{array}$ \\
\hline Outcomes & $\begin{array}{l}\text { - Wound infection definition: CDC criteria. } \\
\text { - Follow-up: } 1,2,12 \text { weeks post operation } \\
\text { - SSSI N infected/N patients (\%): PG: } 4 / 141(2.8 \%) ; \text { CG: } 8 / 141(5.7 \%) \\
\text { - DSSI N infected/N patients (\%): PG: } 0 / 141(0 \%) \text {; CG: } 1 / 141(0.7 \%) \\
\text { - Adverse effects: not reported }\end{array}$ \\
\hline
\end{tabular}


- Funding: Mazandaran University of Medical Sciences

\section{Risk of bias}

\begin{tabular}{|c|c|c|}
\hline Bias & Authors' judgement & Support for judgement \\
\hline $\begin{array}{l}\text { Random sequence genera- } \\
\text { tion (selection bias) }\end{array}$ & Unclear risk & $\begin{array}{l}\text { Quote: "This is a double-blind randomised trial ..." but no further information } \\
\text { on how randomisation was performed was provided. }\end{array}$ \\
\hline $\begin{array}{l}\text { Allocation concealment } \\
\text { (selection bias) }\end{array}$ & Unclear risk & No information available \\
\hline $\begin{array}{l}\text { Blinding of participants } \\
\text { and personnel (perfor- } \\
\text { mance bias) } \\
\text { All outcomes }\end{array}$ & Low risk & $\begin{array}{l}\text { Quote: "This is a double-blind randomised trail ..." } \\
\text { No further information available, but performance bias is unlikely in this set- } \\
\text { ting }\end{array}$ \\
\hline $\begin{array}{l}\text { Blinding of outcome as- } \\
\text { sessment (detection bias) } \\
\text { All outcomes }\end{array}$ & Unclear risk & No information available \\
\hline $\begin{array}{l}\text { Incomplete outcome data } \\
\text { (attrition bias) } \\
\text { All outcomes }\end{array}$ & Low risk & No missing outcome data \\
\hline $\begin{array}{l}\text { Selective reporting (re- } \\
\text { porting bias) }\end{array}$ & High risk & The protocol was registered after conducting the study (irct.ir/trial/1359). \\
\hline Other bias & Low risk & The study appears to be free of other sources of bias \\
\hline
\end{tabular}

Razack 2015

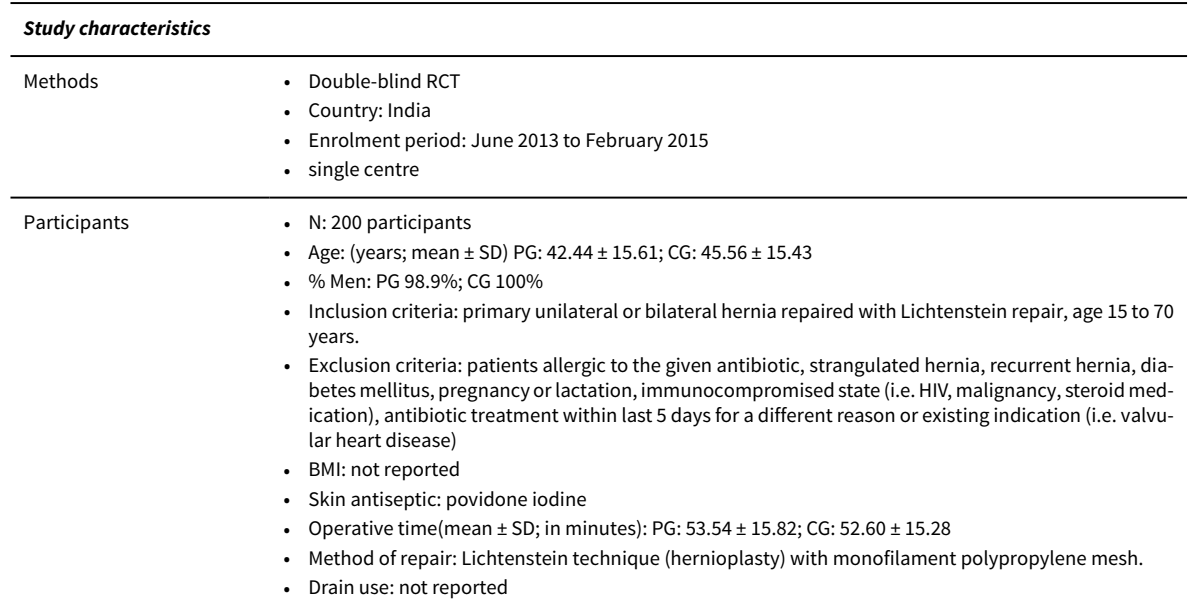


- ASA: PG: ASAI $n=88$ (93.6\%), ASAII $n=6(6.8 \%) ;$ CG: ASAI $n=80(93 \%)$, ASAll $n=6(6.9 \%)$

- Anaesthesia method: not reported

- Hospitalisation method: inpatient

- Length of hospital stay (mean \pm SD, days): PG: $3.9 \pm 3.33$; CG $4.1 \pm 2.07$ (pre-operative stay in days); patients were discharged next day post operation

- Surgeon: not reported

\begin{tabular}{|c|c|}
\hline Interventions & $\begin{array}{l}\text { - Prophylactic group (PG): } \\
\text { - } \mathrm{N}: 94 \\
\text { - } 94 \text { dostibiotic: cefazolin } 1 \mathrm{~g} \\
\text { - number of doses: } 1 \\
\text { - route of administration:intravenous } \\
\text { - timing of administration: just before incision } \\
\text { - Control group (CG): } \\
\text { - } \mathrm{N}: 86 \\
\text { - substance: equal volume of sterile saline }\end{array}$ \\
\hline Outcomes & $\begin{array}{l}\text { - Wound infection definition: CDC criteria/ASEPSIS criteria } \\
\text { - Follow-up: } 2 \text { days, } 1 \text { week, } 4 \text { weeks post operation } \\
\text { - SSSI+DSSI N infected/N patients (\%) PG: } 7 / 94 \text { (7.4\%); CG: } 8 / 86(9.3 \%) \\
\text { - DSSI: } 1 \text { case (not specified in which group) ((reported: "one mesh was removed") } \\
\text { - Adverse effects: not reported }\end{array}$ \\
\hline Notes & $\begin{array}{l}\text { - participants }<18 \text { years old were included in this study } \\
\text { - Staphylococcus aureus, Streptococci, Klebsiella pneumonia, E. coli, enterobacteria were isolated from } \\
\text { the wound infections } \\
\text { - Reported: no financial or competing interest }\end{array}$ \\
\hline
\end{tabular}

\section{Risk of bias}

\begin{tabular}{|c|c|c|}
\hline Bias & Authors' judgement & Support for judgement \\
\hline $\begin{array}{l}\text { Random sequence genera- } \\
\text { tion (selection bias) }\end{array}$ & Unclear risk & $\begin{array}{l}\text { No information available how randomisation was accomplished. } \\
\text { Quote: " } 200 \text { patients were randomized into antibiotic group and control group } \\
\text { by sealed envelope method on the day before the surgery" }\end{array}$ \\
\hline $\begin{array}{l}\text { Allocation concealment } \\
\text { (selection bias) }\end{array}$ & Unclear risk & $\begin{array}{l}\text { Quote: " } 200 \text { patients were randomized into antibiotic group and control group } \\
\text { by sealed envelope method on the day before the surgery" } \\
\text { Not specified whether envelopes were sequentially numbered and opaque. }\end{array}$ \\
\hline $\begin{array}{l}\text { Blinding of participants } \\
\text { and personnel (perfor- } \\
\text { mance bias) } \\
\text { All outcomes }\end{array}$ & Low risk & No information available, but performance bias is unlikely in this setting \\
\hline
\end{tabular}

Blinding of outcome as- Low risk sessment (detection bias) All outcomes
Incomplete outcome data High risk (attrition bias)

All outcomes
Quote: "Surgeon who was not involved in surgery followed the case after 1 week and 4 weeks, postoperatively"
Quote: "The study was concluded in Feb 2015, by then, out of 200 patients who had entered the study, 180 patients had completed one month follow up." "Among the 200 patients with one month follow up, 94 were in the antibiotic group and 86 were in the control group." 
Unclear why not all randomised patients were analysed. The missing outcome data were not balanced in numbers across intervention groups (PG $n=6(6 \%)$; CG $n=14(14 \%))$ and the proportion of missing outcomes compared with the observed event risk is enough to have induced clinically relevant bias in the intervention effect estimate. Available case analysis was performed.

Selective reporting (re- Unclear risk porting bias)
No reporting that study protocol was registered. Authors failed to report the number of cases with SSSI or DSSI per treatment group and therefore metaanalysis for these subgroups is not possible.

Quote: "No significant difference was found between the study groups on analyzing the sub types of infection".

\begin{tabular}{lll}
\hline Other bias & Low risk & Study appears to be free of other sources of bias \\
\hline
\end{tabular}

\begin{tabular}{|c|c|}
\hline \multicolumn{2}{|c|}{ Study characteristics } \\
\hline Methods & $\begin{array}{l}\text { - Double-blind RCT } \\
\text { - Country: India } \\
\text { - Enrolment period: November } 2006 \text { to June } 2008 \\
\text { - Single centre }\end{array}$ \\
\hline Participants & 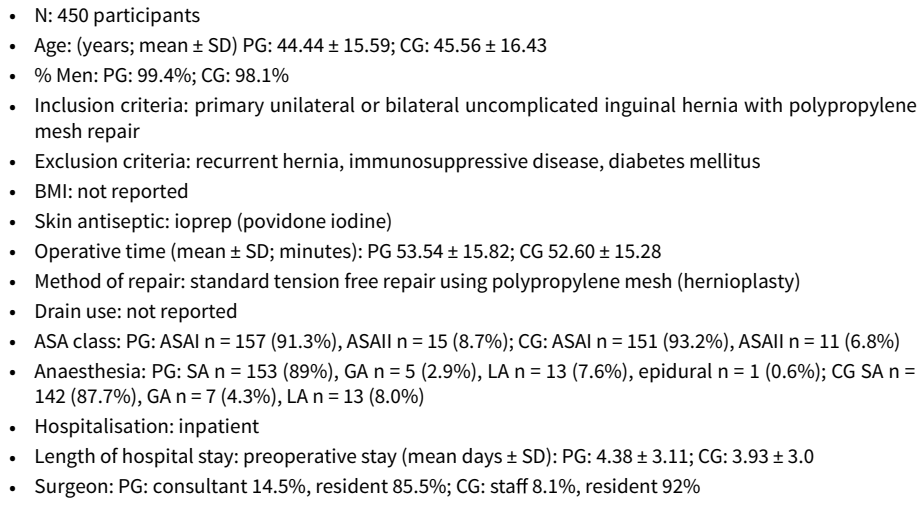 \\
\hline Interventions & $\begin{array}{l}\text { Prophylactic group (PG): } \\
\text { - } \mathrm{N}: 225 \\
\text { - antibiotic: cefazolin } \\
\text { - dose: } 1 \mathrm{~g} \\
\text { - number of doses: } 1 \\
\text { - route of administration: intravenous } \\
\text { - timing of administration: induction } \\
\text { - Control group (CG): } \\
\text { - } \mathrm{N}: 225 \\
\text { - substance: normal saline }\end{array}$ \\
\hline
\end{tabular}




\begin{tabular}{ll} 
- Wutcomes & Wound infection definition: CDC criteria \\
- & Follow-up: 2,7 to 10 and 30 days post operation \\
- & SSSI N infected/N patients (\%): PG:11/172 (7\%); CG: $16 / 162(10.5 \%)$ \\
- & Adverse effects: not reported \\
\hline Notes & Participants < 18 years old were included in this study \\
- Staphylococcus aureus, Streptococci, Klebsiella pneumonia, E. coli, enterobacteria were isolated from & the wound infections \\
- Reported: no conflict of interest. Funding by the institute.
\end{tabular}

\section{Risk of bias}

\begin{tabular}{|c|c|c|}
\hline Bias & Authors' judgement & Support for judgement \\
\hline $\begin{array}{l}\text { Random sequence genera- } \\
\text { tion (selection bias) }\end{array}$ & Unclear risk & $\begin{array}{l}\text { Quote: "After informed consent, } 450 \text { patients were randomized into antibi- } \\
\text { otic group and control group by sealed envelope method on the day before } \\
\text { surgery" } \\
\text { Not specified how randomisation was established }\end{array}$ \\
\hline $\begin{array}{l}\text { Allocation concealment } \\
\text { (selection bias) }\end{array}$ & Unclear risk & $\begin{array}{l}\text { Quote: "After informed consent, } 450 \text { patients were randomized into antibi- } \\
\text { otic group and control group by sealed envelope method on the day before } \\
\text { surgery" } \\
\text { Not specified whether envelopes were sequentially numbered and opaque. }\end{array}$ \\
\hline $\begin{array}{l}\text { Blinding of participants } \\
\text { and personnel (perfor- } \\
\text { mance bias) } \\
\text { All outcomes }\end{array}$ & Low risk & No information available, but performance bias is unlikely in this setting \\
\hline $\begin{array}{l}\text { Blinding of outcome as- } \\
\text { sessment (detection bias) } \\
\text { All outcomes }\end{array}$ & Low risk & $\begin{array}{l}\text { Quoted: "Follow-up was done by residents who where blinded to the drug } \\
\text { used." }\end{array}$ \\
\hline $\begin{array}{l}\text { Incomplete outcome data } \\
\text { (attrition bias) } \\
\text { All outcomes }\end{array}$ & High risk & $\begin{array}{l}\text { Missing outcome data (exclusion of patients from analysis after randomisa- } \\
\text { tion) were reported and are balanced across treatment groups. However, } \\
\text { numbers of missing outcome data are very high (PG } n=53 \text { and CG } n=63 \text { from } \\
n=450 \text { randomised ( } 24 \% \text { to } 26 \%) \text { ). These missing data were excluded from } \\
\text { data analysis and available-case analysis was performed. The proportion of } \\
\text { missing outcomes compared with the observed event risk is enough to have } \\
\text { induced clinically relevant bias in the intervention effect estimate. }\end{array}$ \\
\hline $\begin{array}{l}\text { Selective reporting (re- } \\
\text { porting bias) }\end{array}$ & Low risk & $\begin{array}{l}\text { The study protocol is not available but report includes all expected outcomes, } \\
\text { including specification of SSSI and DSSI }\end{array}$ \\
\hline Other bias & Low risk & The study appears to be free of other sources of bias \\
\hline
\end{tabular}

Taylor 1997

\section{Study characteristics}

\begin{tabular}{ll}
\hline Methods & Double-blind RCT \\
& - Country: UK
\end{tabular}


- Multicentre (6)

\begin{tabular}{|c|c|}
\hline Participants & $\begin{array}{l}\text { - N: } 619 \text { participants } \\
\text { - Age: (years; mean } \pm \text { SD) PG: } 56.7 \pm 17.4 ; \text { CG: } 56.6 \pm 16.5 \\
\text { - Inclusion criteria: inguinal or femoral hernia, age }>18 \text { years } \\
\text { - Exclusion criteria: hypersensitivity to penicillin or cephalosporin, had taken antimicrobial agents with- } \\
\text { in the preceding } 72 \mathrm{~h} \text {, bilateral hernia, pregnant or lactating, renal or liver impairment, had previous } \\
\text { been entered into the study, or patients for whom antibiotic prophylaxis was indicated for some other } \\
\text { reason such as valvular disease. } \\
\text { - BMI: not reported } \\
\text { - Skin antiseptic: betadine or chlorhexidine } \\
\text { - Operative time: not reported } \\
\text { - Method of repair: herniorrhaphy (Bassini or Shouldice or darn, } \mathrm{n}=508 \text { ), hernioplasty (Lichtenstein, } \mathrm{n} \\
\text { - } 33 \text { ), unknown } \mathrm{n}=22 \\
\text { - Drain use: } 4 \text { patients in each group } \\
\text { - ASA class: not reported } \\
\text { - Hospitalisation method: inpatient } \\
\text { - Length of hospital stay: not reported } \\
\text { - Surgeon: Consultant, (senior) registrar/Staff or senior house officer }\end{array}$ \\
\hline Interventions & $\begin{array}{l}\text { - Prophylactic group (PG): } \\
\text { - } \mathrm{N}: 283 \\
\text { - antibiotic: co-amoxicillin clavulanic acid } \\
\text { - dose: } 1.2 \mathrm{~g} \\
\text { - number of doses: } 1 \\
\text { - route of administration: intravenous } \\
\text { - timing of administration: induction or start surgery } \\
\text { - Control group (CG): } \\
\text { - } \mathrm{N}: 280 \\
\text { - substance: equal volume of sterile saline }\end{array}$ \\
\hline
\end{tabular}

Outcomes $\quad$ Wound infection definition: as a purulent wound discharge or spreading erythema indicative of cellulitis, wound breakdown, or dehiscence with clinical evidence of infection.

- Follow-up: 4 to 6 weeks

- SSSI + DSSI N infected/N patients (\%): PG: 25/283 (8.8\%); CG: 25/280 (8.9\%)

- DSSI: not reported

- Adverse effects: not reported

Notes

- S. aureus, S. albus, Streptococci (beta-hemolytic, anaerobic, unspecified), Coliforms, Enterobacter aerogens bacteria were cultured from the wound infections

- Funding, supply of co-amoxiclav antibiotics and statistical analysis assistance by SmithKline Beecham Pharmaceuticals Ltd.

\section{Risk of bias}

Bias Authors' judgement Support for judgement

Random sequence genera- Low risk tion (selection bias)
Quote: "randomization was from a computer-generated code in blocks of four.” 
Taylor 1997 (Continued)

Allocation concealment Unclear risk Insufficient information available (selection bias)

Blinding of participants

Low risk

No information available, but performance bias is unlikely in this setting and personnel (perfor-

mance bias)

All outcomes

\begin{tabular}{|c|c|c|}
\hline $\begin{array}{l}\text { Blinding of outcome as- } \\
\text { sessment (detection bias) } \\
\text { All outcomes }\end{array}$ & Unclear risk & $\begin{array}{l}\text { Unclear whether general practitioner or district nurses were blinded to treat- } \\
\text { ment and therefore it is unclear whether outcome assessment was properly } \\
\text { blinded. } \\
\text { Quote: "Patients were given a diary card on which their general practitioner } \\
\text { or district nurse was asked to record any suggestion of a wound or other infec- } \\
\text { tion..." }\end{array}$ \\
\hline $\begin{array}{l}\text { Incomplete outcome data } \\
\text { (attrition bias) } \\
\text { All outcomes }\end{array}$ & High risk & $\begin{array}{l}\text { Missing outcome data (exclusion of patients from analysis after randomisa- } \\
\text { tion) were reported and seem to be balanced across treatment groups. How- } \\
\text { ever, numbers of missing outcome data are considerablely high ( } \mathrm{n}=56 \text { from } n \\
=619 \text { randomised }(9 \%) \text { ). These data are excluded from data analysis and avail- } \\
\text { able-case analysis was performed. The proportion of missing outcomes com- } \\
\text { pared with the observed event risk is enough to have induced clinically rele- } \\
\text { vant bias in the intervention effect estimate. }\end{array}$ \\
\hline $\begin{array}{l}\text { Selective reporting (re- } \\
\text { porting bias) }\end{array}$ & Low risk & $\begin{array}{l}\text { The study protocol was not available but report includes expected outcome } \\
\text { wound infection. }\end{array}$ \\
\hline Other bias & Low risk & The study appears to be free of other sources of bias \\
\hline
\end{tabular}

Thakur 2010

Study characteristics

\begin{tabular}{|c|c|}
\hline Methods & $\begin{array}{l}\text { - } \text { Double-blind RCT } \\
\text { - Country: India } \\
\text { - Enrolment period: not reported } \\
\text { - Single centre }\end{array}$ \\
\hline Participants & $\begin{array}{l}\text { - N: } 55 \text { participants } \\
\text { - Age: not specified (all adult) } \\
\text { - } \% \text { Men: } 100 \% \\
\text { - Inclusion criteria: unilateral or bilateral elective inguinal hernia } \\
\text { - Exclusion criteria: femoral, strangulated or obstructed hernias; steroid therapy or chemotherapy; had } \\
\text { received antibiotics in the past } 48 \text { hours; valvular heart disease or prosthetic heart valves or joints. } \\
\text { - BMI: not reported } \\
\text { - Skin antiseptic: } 10 \% \text { povidone iodine } \\
\text { - Operative time: PG } 79.3 \% \text { ( }=23 \text { ) had the total duration of surgery > } 1 \text { hour; CG } 92.3 \% \text { ( }=24 \text { ) had } \\
\text { the total duration of surgery }>1 \text { hour. } \\
\text { - Method of repair: Lichtenstein technique using monofilament polypropylene mesh (hernioplasty) } \\
\text { - Drain use: not reported } \\
\text { - AnA class: not reported } \\
\text { - Hospitalisation: not reported } \\
\text { - Length of hospital stay: not reported }\end{array}$ \\
\hline
\end{tabular}




\begin{tabular}{|c|c|}
\hline Interventions & $\begin{array}{l}\text { Prophylactic group (PG): } \\
\text { - } \mathrm{N}: 29 \\
\text { - antibiotic: cefuroxime sodium } \\
\text { - dose: } 1,5 \mathrm{~g} \\
\text { - number of doses: } 1 \\
\text { - route of administration: intravenous } \\
\text { - timing of administration: } 30 \text { minutes before induction } \\
\text { - Control group (CG): } \\
\text { - } \mathrm{N}: 26 \\
\text { - substance: equal volume sterile saline }\end{array}$ \\
\hline Outcomes & $\begin{array}{l}\text { - Wound infection definition: Center of Diseases Control criteria (as described by Teresa and colleagues } \\
\text { 1992) } \\
\text { - Follow-up: discharge, } 1 \text { week, } 1 \text { month } \\
\text { - SSSI N infected/N patients (\%): PG: } 3 / 29(10.3 \%) ; \text { CG: } 3 / 26(11.5 \%) \\
\text { - DSSI N infected/N patients (\%): PG: } 0 / 29(0 \%) ; C G: 1 / 26(3.8 \%) \\
\text { - Adverse effects: not reported }\end{array}$ \\
\hline Notes & $\begin{array}{l}\text { - S. aureus bacteria were cultured from the wound infections } \\
\text { - Funding is not reported. }\end{array}$ \\
\hline
\end{tabular}

\section{Risk of bias}

Bias Authors' judgement Support for judgement

Random sequence genera- Low risk No information on how randomisation was accomplished. However, the block tion (selection bias) randomisation method indicates that the randomisation methodology has been performed carefully to minimise bias.

Quote: "Randomization was accomplished by block randomization”.

\begin{tabular}{|c|c|c|}
\hline $\begin{array}{l}\text { Allocation concealment } \\
\text { (selection bias) }\end{array}$ & Unclear risk & No information available \\
\hline $\begin{array}{l}\text { Blinding of participants } \\
\text { and personnel (perfor- } \\
\text { mance bias) } \\
\text { All outcomes }\end{array}$ & Low risk & No information available, but performance bias is unlikely in this setting \\
\hline $\begin{array}{l}\text { Blinding of outcome as- } \\
\text { sessment (detection bias) } \\
\text { All outcomes }\end{array}$ & Unclear risk & No information available \\
\hline $\begin{array}{l}\text { Incomplete outcome data } \\
\text { (attrition bias) } \\
\text { All outcomes }\end{array}$ & Low risk & No missing outcome data \\
\hline $\begin{array}{l}\text { Selective reporting (re- } \\
\text { porting bias) }\end{array}$ & Low risk & $\begin{array}{l}\text { The study protocol is not available but report includes all expected outcomes, } \\
\text { including specification of SSSI and DSSI }\end{array}$ \\
\hline Other bias & Low risk & The study appears to be free of other sources of bias \\
\hline
\end{tabular}




\begin{tabular}{|c|c|}
\hline Methods & $\begin{array}{l}\text { - } \text { triple blind RCT } \\
\text { - Country: Greece } \\
\text { - Enrolment period: January } 2000 \text { and June } 2004 \\
\text { - Single centre }\end{array}$ \\
\hline Participants & 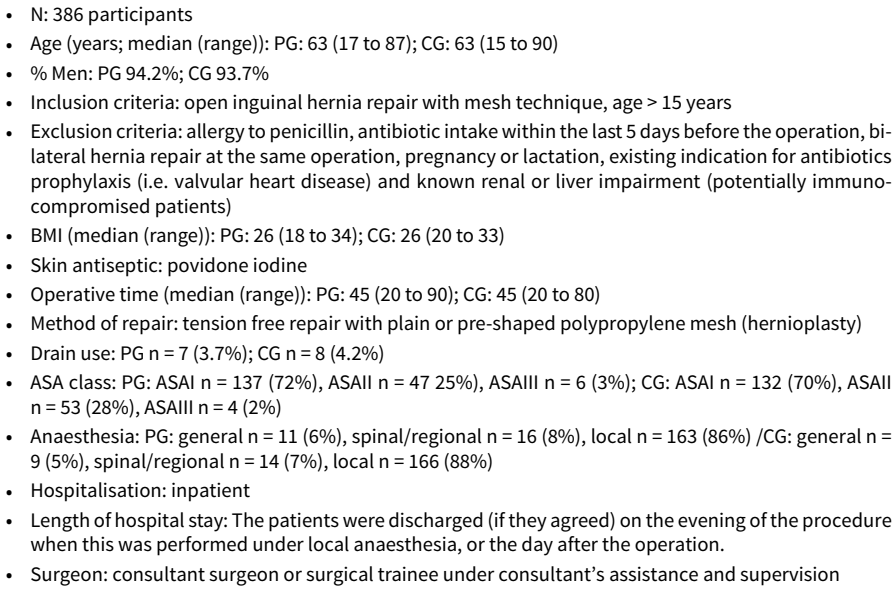 \\
\hline Interventions & $\begin{array}{l}\text { - Prophylactic group (PG): } \\
\text { N: } 193 \\
\text { - antibiotic: ampicillin + clavoulanic acid } \\
\text { - dose: } 1.2 \mathrm{~g} \\
\text { - number of doses: } 1 \\
\text { - route of administration: intravenous } \\
\text { - timing of administration: induction. } \\
\text { - Control group (CG): } \\
\text { - N: } 193 \\
\text { - substance: equal volume sterile saline }\end{array}$ \\
\hline
\end{tabular}

- Wound infection definition: purulent discharge (or serosanguineous with positive culture) or spreading erythema indicative of cellulitis or wound breakdown/dehiscence with clinical evidence of infection

- Follow-up: 1 week, 1 month after operation

- SSSI N infected/N patients (\%): PG: 5/190 (2.6\%); CG: $9 / 189(4.8 \%)$

- DSSI N infected/N patients: not reported (mesh removal was not required in any case of wound infection)

- Adverse effects: not reported

- S. aureus, S. epidermidis, Streptococcus beta-haemolytic bacteria were cultured from wound infections 


\begin{tabular}{|c|c|c|}
\hline \multicolumn{3}{|l|}{ Risk of bias } \\
\hline Bias & Authors' judgement & Support for judgement \\
\hline $\begin{array}{l}\text { Random sequence genera- } \\
\text { tion (selection bias) }\end{array}$ & Low risk & $\begin{array}{l}\text { Quote: "The list with code numbers generated by the Arcus Quistat software } \\
\text { program was kept by a secretary not involved in the treatment of the patients } \\
\text { at any stage and was opened at the end of the trial for analysis of the results" }\end{array}$ \\
\hline $\begin{array}{l}\text { Allocation concealment } \\
\text { (selection bias) }\end{array}$ & Unclear risk & $\begin{array}{l}\text { Not specified whether envelopes were sequentially numbered and opaque. } \\
\text { Quote: "Patients were randomized ....using numbered sealed envelopes" }\end{array}$ \\
\hline $\begin{array}{l}\text { Blinding of participants } \\
\text { and personnel (perfor- } \\
\text { mance bias) } \\
\text { All outcomes }\end{array}$ & Low risk & $\begin{array}{l}\text { Quote: "the agent (antibiotic of placebo)was prepared by the anesthetic nurse } \\
\text { and neither the surgeon or the patient was aware of the randomization arm" }\end{array}$ \\
\hline $\begin{array}{l}\text { Blinding of outcome as- } \\
\text { sessment (detection bias) } \\
\text { All outcomes }\end{array}$ & Low risk & $\begin{array}{l}\text { Quote: "They were followed-up as outpatients at } 1 \text { week and } 1 \text { month after the } \\
\text { operation by one of the authors who was not involved in surgery and therefore } \\
\text { was blind to the randomization arm" }\end{array}$ \\
\hline \multirow[t]{2}{*}{$\begin{array}{l}\text { Incomplete outcome data } \\
\text { (attrition bias) } \\
\text { All outcomes }\end{array}$} & Low risk & $\begin{array}{l}\text { A small number of patients is lost-in-follow-up (PG } n=3(1.6 \%) \text {; CG } n=(2.1 \%) \text { ). } \\
\text { Missing data were balanced between treatment groups and reasons for miss- } \\
\text { ing outcome data unlikely to be related to the true outcome. The proportion } \\
\text { of missing outcomes compared with the observed event risk is not enough to } \\
\text { have had a clinically relevant impact on the intervention effect estimate. Avail- } \\
\text { able case analysis was performed. }\end{array}$ \\
\hline & & $\begin{array}{l}\text { Quote: "Three group A patients and four group B patients were excluded be- } \\
\text { cause they had inadvertent medical administration }(n=3) \text {, were lost-to-fol- } \\
\text { low-up }(n=2) \text {, were found to have unexpected femoral hernia }(n=1) \text { or had } \\
\text { hernia repair without mesh insertion }(n=1) \text { " }\end{array}$ \\
\hline $\begin{array}{l}\text { Selective reporting (re- } \\
\text { porting bias) }\end{array}$ & Low risk & $\begin{array}{l}\text { The study protocol is not available but report includes all expected outcomes, } \\
\text { including specification of SSSI. }\end{array}$ \\
\hline Other bias & Low risk & The study appears to be free of other sources of bias \\
\hline
\end{tabular}

Ullah 2013

\begin{tabular}{ll}
\hline Study characteristics & \\
\hline Methods & single blind RCT \\
& - Country: Pakistan \\
& - Enrolment period: January to December 2012 \\
& - Single centre \\
\hline Participants & N: 166 participants \\
& - $\%$ Mge: (years; mean \pm SD): PG: $54.33 \pm 11.77 ;$ CG: $52.58 \pm 11.80$ \\
& Inclusion criteria: unilateral inguinal hernia, male patients, age $>18$ years \\
& $\begin{array}{l}\text { Exclusion criteria: diabetes (fasting glucose }>126 \text { mg/dl), history of intake of steroids in last } 2 \text { weeks, } \\
\text { renal function }\end{array}$
\end{tabular}


- BMI: not reported

- Skin antiseptic: not reported

- Operative time: not reported

- Method of repair: Lichtenstein technique with polypropylene mesh (hernioplasty)

- Drain use: not reported

- ASA class: not reported

- Anaesthesia: not reported

- Hospitalisation: inpatient

- Length of hospital stay: 2 days

- Surgeon: a single general surgeon

\begin{tabular}{ll}
\hline Interventions & Prophylactic group (PG): \\
& - $\mathrm{N}: 83$ \\
& - antibiotic: co-amoxiclav \\
& - dose: $1 \mathrm{~g}$ \\
& - number of doses: 1 \\
& - route of administration: not reported \\
& - timing of administration: 1 hour before intervention \\
& Control group $(\mathrm{CG}):$ \\
& - $\mathrm{N}: 83$ \\
& - substance: placebo (not specified)
\end{tabular}

\begin{tabular}{ll}
\hline Outcomes & Wound infection definition: not reported \\
& - Follow-up: 2 days, 14 days after operation after operation \\
& $-\mathrm{SSSI} N$ infected/N patients $(\%): \mathrm{PG}: 6 / 83(7.2 \%) ; \mathrm{CG}: 15 / 83(18.1 \%)$ \\
& $-\mathrm{DSSI}$ : not reported \\
& - Adverse effects: not reported
\end{tabular}

\begin{tabular}{|c|c|c|}
\hline Notes & \multicolumn{2}{|l|}{ Funding is not reported. } \\
\hline \multicolumn{3}{|l|}{ Risk of bias } \\
\hline Bias & Authors' judgement & Support for judgement \\
\hline $\begin{array}{l}\text { Random sequence genera- } \\
\text { tion (selection bias) }\end{array}$ & Low risk & $\begin{array}{l}\text { No information on how randomisation was accomplished. However, the block } \\
\text { randomisation method indicates that the randomisation methodology has } \\
\text { been performed carefully to minimise bias. }\end{array}$ \\
\hline & & $\begin{array}{l}\text { Quote: "The patients diagnosed as inguinal hernia were taken through OPD, } \\
\text { and were randomly placed into } 2 \text { groups using block method of randomisation } \\
\text { (block size of 4)." }\end{array}$ \\
\hline $\begin{array}{l}\text { Allocation concealment } \\
\text { (selection bias) }\end{array}$ & Unclear risk & No information available. \\
\hline $\begin{array}{l}\text { Blinding of participants } \\
\text { and personnel (perfor- } \\
\text { mance bias) }\end{array}$ & Low risk & $\begin{array}{l}\text { No information on patient blinding available, but performance bias is unlikely } \\
\text { in this setting }\end{array}$ \\
\hline All outcomes & & $\begin{array}{l}\text { Quote: "All patients in both groups were put on OT list for the next OT day after } \\
\text { following routine and standard preoperative preparations kept uniform for all } \\
\text { participants." }\end{array}$ \\
\hline $\begin{array}{l}\text { Blinding of outcome as- } \\
\text { sessment (detection bias) } \\
\text { All outcomes }\end{array}$ & Unclear risk & No information available. \\
\hline
\end{tabular}


Ullah 2013 (Continued)

Incomplete outcome data Low risk

No missing outcome data. (attrition bias)

All outcomes

\begin{tabular}{|c|c|c|}
\hline $\begin{array}{l}\text { Selective reporting (re- } \\
\text { porting bias) }\end{array}$ & Low risk & $\begin{array}{l}\text { The study protocol was not available but report includes all expected out- } \\
\text { comes, including specification of SSSI }\end{array}$ \\
\hline
\end{tabular}

Other bias Low risk The study appears to be free of other sources of bias

Wang 2013

\section{Study characteristics}

\begin{tabular}{|c|c|}
\hline Methods & $\begin{array}{l}\text { - Double-blind RCT } \\
\text { - Country: China } \\
\text { - Enrolment period: January } 2010 \text { to July } 2011 \\
\text { - Multicentre (6) }\end{array}$ \\
\hline Participants & 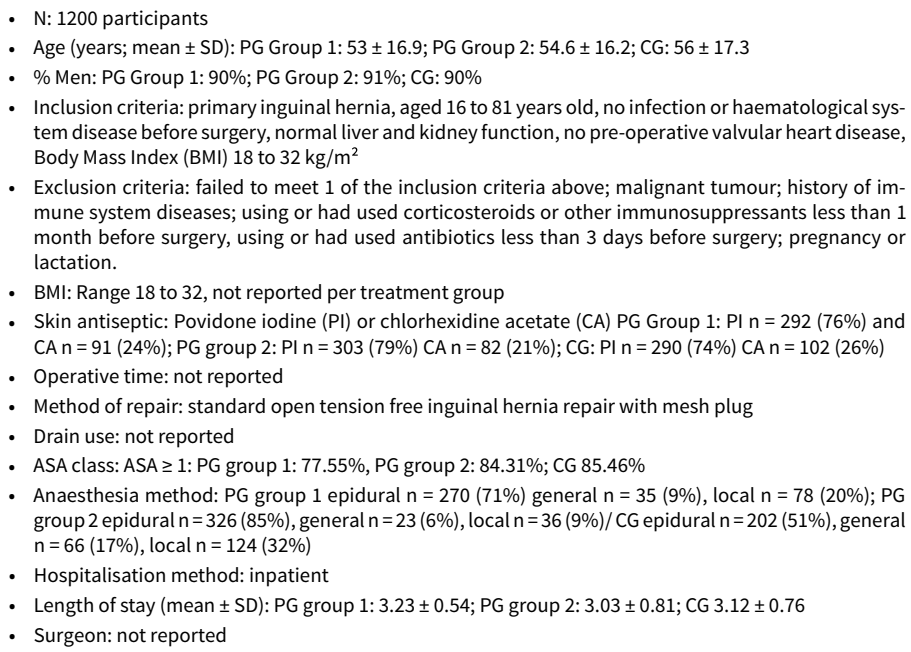 \\
\hline Interventions & $\begin{array}{l}\text { Prophylactic group (PG): } \\
\text { - N: } 800 \text { (Group 1: } 400 \text {; Group 2: } 400 \text { ) } \\
\text { - antibiotic: cefazolin (Group 1); levofloxacin (Group 2) } \\
\text { - dose: group 1: } 1 \mathrm{~g} \text {; group 2: } 200 \mathrm{mg} \\
\text { - number of doses: } 1 \\
\text { - route of administration: intravenous } \\
\text { - timing of administration: } 30 \text { to } 60 \text { minutes before operation } \\
\text { - Control group (CG): } \\
\text { - N: } 400 \\
\text { - substance: equal volume of sterile saline }\end{array}$ \\
\hline
\end{tabular}


- Follow-up: 1 week, 2 weeks, 3 weeks up to 30 days after operation.

- SSSI N infected/N patients (\%): PG: (group $1+$ group 2) = $29(13+16) / 768(3,8 \%) ;$ CG: 19/392 (4.8\%)

- DSSI N infected/N patients (\%): PG (group 1+ group 2) =3 (2+1)/768 (0.4\%); CG: $1 / 392$ (0.3\%) (reported: "one mesh was removed")

- Adverse effects: 1 participant in the cefazolin treatment group and 5 participants in the levofloxacin treatment group failed to tolerate antibiotics

- Participants age $<18$ years old were included in this study

- Mainly Staphylococcus aureus, Staphylococcus epidermidis (skin) and Escherichia coli, Enterococci, Enterococcus faecalis (gut) bacteria were cultured from infections. Also Acinobacter baumanii, Candida tropicalis, Enterobacter cloacae, Haemolytic streptococcus, Klebsiella pneumoniae, Candida albicans, MRSA, Pseudomonas aeruginosa, bacteria were cultured from infections (incl. wound, urinary tract and pulmonary and mesh infections)

- Reported is: no conflict of interest

\section{Risk of bias}

\begin{tabular}{|c|c|c|}
\hline Bias & Authors' judgement & Support for judgement \\
\hline \multirow[t]{2}{*}{$\begin{array}{l}\text { Random sequence genera- } \\
\text { tion (selection bias) }\end{array}$} & Low risk & $\begin{array}{l}\text { Quote: "Allocation was accomplished .....with a computer randomization pro- } \\
\text { gram (Randomization }\end{array}$ \\
\hline & & Adviser 1.0)" \\
\hline $\begin{array}{l}\text { Allocation concealment } \\
\text { (selection bias) }\end{array}$ & Low risk & $\begin{array}{l}\text { Quote: "Allocation was accomplished by a resident who was unaware of this } \\
\text { trial and was not involved in surgery, data collection, follow-up or the analy- } \\
\text { sis of results with a computer randomization program (Randomization Adviser } \\
\text { 1.0)" }\end{array}$ \\
\hline $\begin{array}{l}\text { Blinding of participants } \\
\text { and personnel (perfor- } \\
\text { mance bias) }\end{array}$ & Low risk & $\begin{array}{l}\text { Quote: "Drugs and placebo were prepared by a surgery nurse without previous } \\
\text { knowledge of this trial." }\end{array}$ \\
\hline All outcomes & & $\begin{array}{l}\text { Quote: "On all patients a standard open tension-free inguinal hernia repair op- } \\
\text { eration was performed by surgeons-in-charge (blinded) using a polypropylene } \\
\text { mesh" }\end{array}$ \\
\hline $\begin{array}{l}\text { Blinding of outcome as- } \\
\text { sessment (detection bias) } \\
\text { All outcomes }\end{array}$ & Low risk & $\begin{array}{l}\text { Quote: "The main endpoint was the amount of post-operation infection pa- } \\
\text { tients evaluated by surgeons and nurses (blinded) within } 30 \text { days after opera- } \\
\text { tion." }\end{array}$ \\
\hline \multirow{3}{*}{$\begin{array}{l}\text { Incomplete outcome data } \\
\text { (attrition bias) } \\
\text { All outcomes }\end{array}$} & Low risk & $\begin{array}{l}\text { A small number of participants was lost-in-follow-up (PG } n=32(4 \%) ; C G n=8 \\
(2 \%)) \text {. }\end{array}$ \\
\hline & & $\begin{array}{l}\text { Missing data were not balanced across intervention groups, but the proportion } \\
\text { of missing outcomes compared with the observed event risk is not enough to } \\
\text { have had a clinically relevant impact on the intervention effect estimate. }\end{array}$ \\
\hline & & Available-case analysis was performed. \\
\hline $\begin{array}{l}\text { Selective reporting (re- } \\
\text { porting bias) }\end{array}$ & Low risk & $\begin{array}{l}\text { The protocol was registered (NCT 01802606) and study outcomes that are of } \\
\text { interest in the review have been reported in the prespecified way. }\end{array}$ \\
\hline Other bias & Low risk & The study appears to be free of other sources of bias \\
\hline
\end{tabular}




\section{Study characteristics}

\begin{tabular}{|c|c|}
\hline Methods & $\begin{array}{l}\text { - Triple-blind RCT } \\
\text { - Country: Turkey } \\
\text { - Enrolment period: between September } 1996 \text { and July } 1998 \\
\text { - Single centre }\end{array}$ \\
\hline Participants & 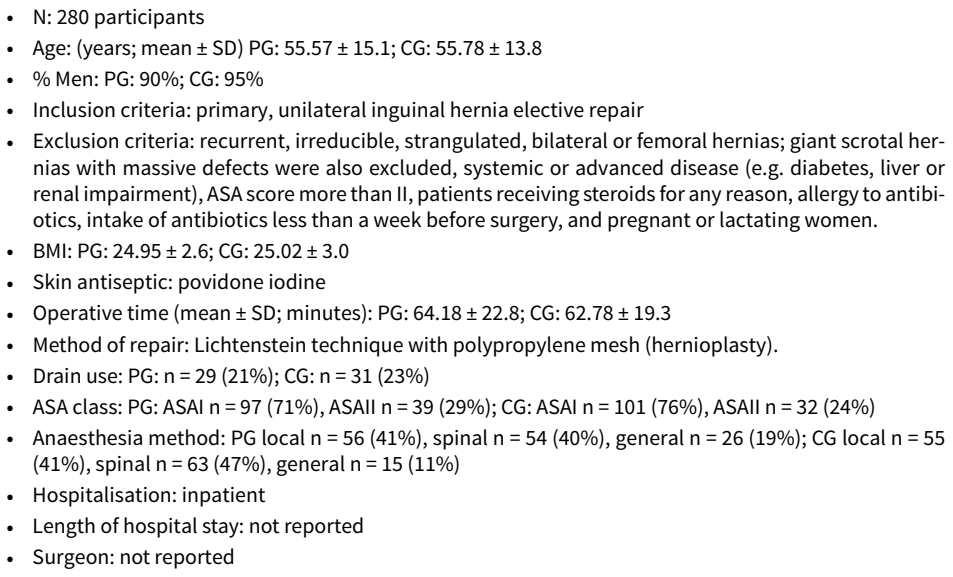 \\
\hline Interventions & $\begin{array}{l}\text { - Prophylactic group (PG): } \\
\text { - } \mathrm{N}: 140 \\
\text { - antibiotic: ampicillin-sulbactam } \\
\text { - dose: } 1.5 \mathrm{~g}(1 \mathrm{~g}+0.5 \mathrm{~g}) \\
\text { - number of doses: } 1 \\
\text { - route of administration: intravenous } \\
\text { - timing of administration: before incision } \\
\text { - Control group (CG): } \\
\text { - } \mathrm{N}: 140 \\
\text { - substance: equal volume sterile saline }\end{array}$ \\
\hline Outcomes & $\begin{array}{l}\text { - Wound infection definition: CDC criteria } \\
\text { - Follow-up: discharge, } 7 \text { to } 9 \text { days and } 4 \text { to } 6 \text { weeks, } 6 \text { months, } 12 \text { months post operation } \\
\text { - SSSI N infected/N patients (\%): PG: } 0 / 136(0 \%) ; C G: 9 / 133(6.8 \%) \\
\text { - DSSI N infected/N patients (\%): PG: } 1 / 136(0.7 \%) ; C G: 3 / 133(2.3 \%) \text { (reported: "three meshes were re- } \\
\text { moved") } \\
\text { - Adverse effects: not reported }\end{array}$ \\
\hline Notes & $\begin{array}{l}\text { - S. aureus and S. epidermidis bacteria were cultured from wound infections. } \\
\text { - Funding is not reported. }\end{array}$ \\
\hline \multicolumn{2}{|l|}{ Risk of bias } \\
\hline Bias & Support for judgement \\
\hline
\end{tabular}




\begin{tabular}{ll}
\hline $\begin{array}{l}\text { Allocation concealment } \\
\text { (selection bias) }\end{array}$ & $\begin{array}{l}\text { Quote: "Randomisation was accomplished ....by a resident..... He was unaware } \\
\text { of the research in progress and was never involved in surgery, data collection } \\
\text { or patient follow-up" }\end{array}$
\end{tabular}

\begin{tabular}{ll}
\hline $\begin{array}{l}\text { Blinding of participants } \\
\text { and personnel (perfor- }\end{array}$ & Quote: “...by a resident who also prepared the sealed antibiotic or placebo sy- \\
mance bias) & ringes. He was unaware of the research in progress ...” \\
All outcomes &
\end{tabular}

\begin{tabular}{|c|c|c|}
\hline $\begin{array}{l}\text { Blinding of outcome as- } \\
\text { sessment (detection bias) }\end{array}$ & Low risk & $\begin{array}{l}\text { Quote: "A surgeon masked to the randomization and patient details evaluated } \\
\text { the wound during each follow-up." }\end{array}$ \\
\hline
\end{tabular}

All outcomes

Incomplete outcome data Low risk
(attrition bias)

A small number of patients were lost to follow-up (PG $n=4(2.9 \%) ; C G n=7$ (attrition bias) $(5 \%))$. Missing data were not balanced across intervention groups, but the proportion of missing outcomes compared with the observed event risk is not enough to have had a clinically relevant impact on the intervention effect estimate. Available-case analysis was performed.

Quote: "All patients lost-to-follow-up were contacted by phone and none had any wound problems during the year after surgery"

\begin{tabular}{|c|c|c|}
\hline $\begin{array}{l}\text { Selective reporting (re- } \\
\text { porting bias) }\end{array}$ & Low risk & $\begin{array}{l}\text { The study protocol is not available but report includes all expected outcomes, } \\
\text { including specification of both SSSI and DSSI }\end{array}$ \\
\hline \multirow[t]{2}{*}{ Other bias } & High risk & $\begin{array}{l}\text { Study was terminated early after interim analysis, and therefore there might } \\
\text { be risk of bias for increased benefit of in the antibiotics prophylaxis group. }\end{array}$ \\
\hline & & $\begin{array}{l}\text { Quote: "Because of the high rate of wound infections, the code was broken af- } \\
\text { ter the discharge of patient } 280 \text { ( } 140 \text { patients in the placebo and } 140 \text { patients } \\
\text { in the antibiotic group). At that point, the results revealed an extreme discrep- } \\
\text { ancy in favour of antibiotics prophylaxis and therefore the study was prema- } \\
\text { turely stopped before reaching the previously established sample size of 334" }\end{array}$ \\
\hline
\end{tabular}

$\mathrm{PG}=$ prophylaxis group, $\mathrm{CG}=$ control (placebo) group, $\mathrm{SD}=$ standard deviation

Characteristics of excluded studies [ordered by study ID]

\begin{tabular}{ll}
\hline Study & Reason for exclusion \\
\hline Ahmed 2014 & $\begin{array}{l}\text { Considered plagiarism of published report of Goyal 2011. Results section and large text fragments } \\
\text { of Ahmed } 2014 \text { were similar to Goyal et al.. The study by Goyal and colleagues was published in } \\
2011 \text { and the study by Ahmed and colleagues was published in 2014, therefore Ahmed 2014 was } \\
\text { considered plagiarism and excluded from analysis. }\end{array}$ \\
\hline Aufenacker 2006 & Wrong study design. This is a systematic review and meta-analysis. \\
\hline Barreca 2000 & Wrong study design; non-randomised study. \\
\hline Bhuiyan 2017 & This is an abstract for the ASGBI conference.
\end{tabular}




\begin{tabular}{|c|c|}
\hline Study & Reason for exclusion \\
\hline Boonchan 2017 & Wrong study design. This is a systematic review and meta-analysis. \\
\hline Erdas 2016 & $\begin{array}{l}\text { Wrong study design. This is a systematic review and meta-analysis. Cross-referencing resulted in } 2 \\
\text { additional RCTs that were included in this review and meta-analysis. }\end{array}$ \\
\hline Esposito 2006 & $\begin{array}{l}\text { Incomplete data. Study focused on clean surgical techniques including hernia repair, but data ex- } \\
\text { traction for this subgroup of participants was not possible }\end{array}$ \\
\hline Gierhake 1975 & Wrong study design; non-randomised study. \\
\hline Lazorthes 1993 & Duplicate publication in French of similar study population of Lazorthes 1992 \\
\hline Leon 2011 & Wrong study design; non-randomised study. \\
\hline Li 2012 & Wrong study design. This is a systematic review and meta-analysis. \\
\hline Mazaki 2013b & Wrong study design. This is a systematic review and meta-analysis. \\
\hline Mehrabi Bahar 2015 & $\begin{array}{l}\text { Incomplete data. Study focused on clean surgical techniques including hernia repair, but data ex- } \\
\text { traction for this subgroup of participants was not possible. }\end{array}$ \\
\hline Pessaux 2006 & $\begin{array}{l}\text { Prognostic study based on } 3 \text { randomised studies that compared antibiotics prophylaxis versus an- } \\
\text { other antibiotics prophylaxis regime. }\end{array}$ \\
\hline Sanabria 2007 & Wrong study design. This is a systematic review and meta-analysis. \\
\hline Sanchez-Manuel 2003 & Wrong study design. This is a systematic review and meta-analysis. \\
\hline Sanchez-Manuel 2012 & Wrong study design. This is a systematic review and meta-analysis. \\
\hline Taylor 1996 & Wrong study design; non-randomised study. \\
\hline Yin 2012 & Wrong study design. This is a systematic review and meta-analysis. \\
\hline
\end{tabular}

Characteristics of studies awaiting classification [ordered by study ID]

Barkhordary 2012

\begin{tabular}{ll}
\hline Methods & Double-blind RCT \\
\hline Participants & elective hernioplasty with polypropylene mesh ( $\mathrm{n}=100$ adult male participants) \\
\hline Interventions & $1 \mathrm{~g}$ cefazolin (IV) versus sterile saline (placebo) \\
\hline Outcomes & surgical site infections, follow-up at 1 week, 6 to 8 weeks and 12 weeks postoperatively \\
\hline Notes & IRCT2012090210719N1; registered while recruiting \\
\hline
\end{tabular}


DATA AND ANALYSES

Comparison 1. Wound infections herniorrhaphy

\begin{tabular}{lllll}
\hline Outcome or subgroup title & $\begin{array}{l}\text { No. of } \\
\text { studies }\end{array}$ & $\begin{array}{l}\text { No. of } \\
\text { partici- } \\
\text { pants }\end{array}$ & Statistical method & Effect size \\
\hline 1.1 All wound infections (SSSI+DSSI) & 5 & 1865 & Risk Ratio (M-H, Random, 95\% Cl) & $0.86[0.56,1.33]$ \\
\hline 1.1 .1 Low infection risk environment & 4 & 1302 & Risk Ratio (M-H, Random, 95\% Cl) & $0.63[0.28,1.41]$ \\
\hline 1.1 .2 High infection risk environment & 1 & 563 & Risk Ratio (M-H, Random, 95\% Cl) & $0.99[0.58,1.68]$ \\
\hline
\end{tabular}

Analysis 1.1. Comparison 1: Wound infections herniorrhaphy, Outcome 1: All wound infections (SSSI+DSSI)

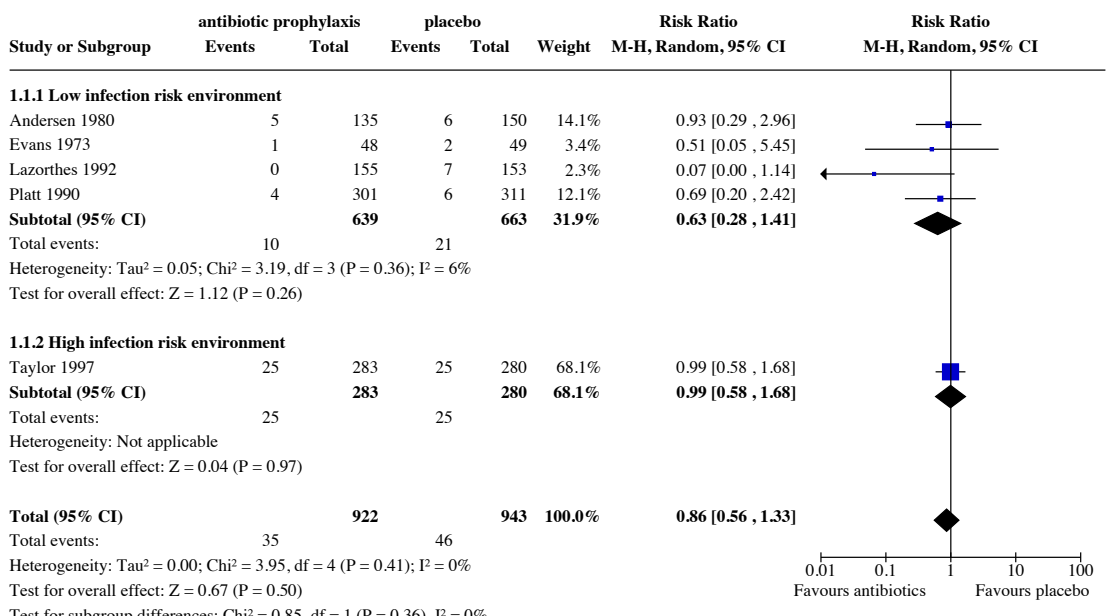

\section{Comparison 2. Wound infections hernioplasty}

\begin{tabular}{|c|c|c|c|c|}
\hline Outcome or subgroup title & $\begin{array}{l}\text { No. of } \\
\text { studies }\end{array}$ & $\begin{array}{l}\text { No. of } \\
\text { partici- } \\
\text { pants }\end{array}$ & Statistical method & Effect size \\
\hline 2.1 All wound infections (SSSI+DSSI) & 22 & 6443 & Risk Ratio (M-H, Random, 95\% Cl) & $0.61[0.48,0.78]$ \\
\hline 2.1.1 low infection risk environment & 9 & 3100 & Risk Ratio (M-H, Random, 95\% Cl) & $0.71[0.44,1.14]$ \\
\hline 2.1.2 high infection risk environment & 13 & 3343 & Risk Ratio (M-H, Random, 95\% Cl) & $0.58[0.43,0.77]$ \\
\hline $\begin{array}{l}\text { 2.2 Superficial Surgical Site Infections } \\
\text { (SSSI) }\end{array}$ & 21 & 6263 & Risk Ratio (M-H, Random, 95\% Cl) & $0.60[0.46,0.78]$ \\
\hline
\end{tabular}




\begin{tabular}{llllll}
\hline Outcome or subgroup title & $\begin{array}{l}\text { No. of } \\
\text { studies }\end{array}$ & $\begin{array}{l}\text { No. of } \\
\text { partici- } \\
\text { pants }\end{array}$ & Statistical method & Effect size \\
\hline 2.2 .1 low infection risk environment & 9 & 3100 & Risk Ratio (M-H, Random, 95\% Cl) & $0.71[0.44,1.17]$ \\
\hline 2.2 .2 high infection risk environment & 12 & 3163 & Risk Ratio (M-H, Random, 95\% Cl) & $0.56[0.41,0.77]$ \\
\hline 2.3 Deep Surgical Site Infections (DSSI) & 12 & 4185 & Risk Ratio (M-H, Random, 95\% Cl) & $0.65[0.26,1.65]$ \\
\hline 2.3 .1 low infection risk environment & 3 & 1488 & Risk Ratio (M-H, Random, 95\% Cl) & $0.67[0.11,4.13]$ \\
\hline 2.3.2 high infection risk environment & 9 & 2697 & Risk Ratio (M-H, Random, 95\% Cl) & $0.64[0.22,1.89]$ \\
\hline
\end{tabular}


Analysis 2.1. Comparison 2: Wound infections hernioplasty, Outcome 1: All wound infections (SSSI+DSSI)

Prophylaxis Events
Control

Total
Events Total
Risk Ratio

Weight M-H, Random, 95\% CI
Risk Ratio

M-H, Random, 95\% CI
2.1.1 low infection risk environment

Aufenacker 2004

Bidhur 2013

Goyal 2011

Jain 2008

Kochhar 2014

Morales 2000

Oteiza 2004

Perez 2005

Tzovaras 2007

Subtotal $(95 \%$ CI)

Total events:

Heterogeneity: $\mathrm{Tau}^{2}=0.00 ; \mathrm{Chi}^{2}=2.12, \mathrm{df}=8(\mathrm{P}=0.98) ; \mathrm{I}^{2}=0 \%$

Test for overall effect: $\mathrm{Z}=1.41(\mathrm{P}=0.16)$

\subsection{2 high infection risk environment}

Al-Fatah 2011

Celdran 2004

Ergul 2011

Ijaz 2010

Mazaki 2013

Othman 2011

Rahmani 2012

Razack 2015

Shankar 2010

Thakur 2010

Ullah 2013

Wang 2013

Yerdel 2001

Subtotal (95\% CI)

Total events:

Heterogeneity: $\mathrm{Tau}^{2}=0.00 ; \mathrm{Chi}^{2}=11.84, \mathrm{df}=12(\mathrm{P}=0.46) ; \mathrm{I}^{2}=0 \%$

Test for overall effect: $Z=3.73(P=0.0002)$

\section{Total $(95 \%$ CI $)$}

3399

$3044100.0 \%$

Total events:

109

166

Heterogeneity: $\mathrm{Tau}^{2}=0.00 ; \mathrm{Chi}^{2}=14.37, \mathrm{df}=21(\mathrm{P}=0.85) ; \mathrm{I}^{2}=0 \%$

Test for overall effect: $Z=3.92(\mathrm{P}<0.0001)$

Test for subgroup differences: $\mathrm{Chi}^{2}=0.52, \mathrm{df}=1(\mathrm{P}=0.47), \mathrm{I}^{2}=0 \%$
$0.89[0.35,2.29]$

$0.33[0.01,7.87]$

$0.33[0.04,3.15]$

$1.00[0.06,15.62]$

$0.80[0.22,2.90]$

$0.80[0.23,2.82]$

$2.98[0.12,72.35]$

$0.57[0.17,1.92]$

$0.55[0.19,1.62]$

$0.71[0.44,1.14]$
$0.60[0.15,2.44]$

$0.11[0.01,1.97]$

$0.71[0.23,2.18]$

$0.40[0.08,1.97]$

$0.15[0.04,0.66]$

$0.64[0.19,2.13]$

$0.44[0.14,1.41]$

$0.80[0.30,2.11]$

$0.66[0.33,1.35]$

$0.67[0.17,2.73]$

$0.40[0.16,0.98]$

$0.82[0.47,1.41]$

$0.08[0.01,0.62]$

$0.58[0.43,0.77]$
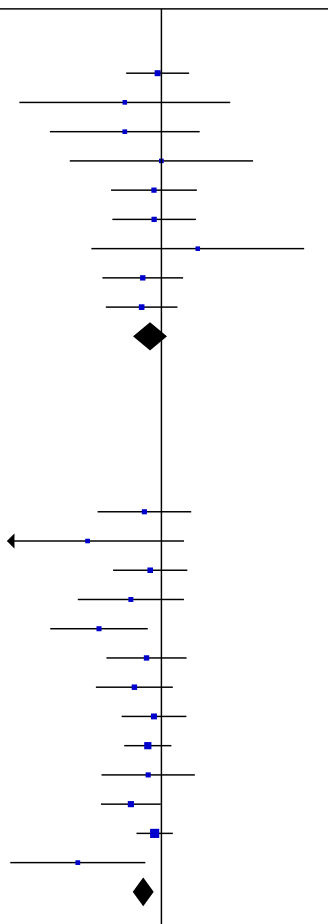

$0.61[0.48,0.78]$

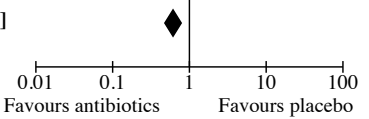


Analysis 2.2. Comparison 2: Wound infections hernioplasty, Outcome 2: Superficial Surgical Site Infections (SSSI)

\begin{tabular}{|c|c|c|c|c|c|}
\hline \multirow[b]{2}{*}{ Study or Subgroup } & \multicolumn{2}{|c|}{ Antibiotic prophylaxis } & \multicolumn{2}{|c|}{ Control } & \multirow[b]{2}{*}{ Weight } \\
\hline & Events & Total & Events & Total & \\
\hline \multicolumn{6}{|c|}{ 2.2.1 low infection risk environment } \\
\hline Aufenacker 2004 & 7 & 503 & 7 & 505 & $6.4 \%$ \\
\hline Bidhur 2013 & 0 & 30 & 1 & 30 & $0.7 \%$ \\
\hline Goyal 2011 & 1 & 100 & 3 & 100 & $1.4 \%$ \\
\hline Jain 2008 & 1 & 60 & 1 & 60 & $0.9 \%$ \\
\hline Kochhar 2014 & 4 & 106 & 5 & 106 & $4.2 \%$ \\
\hline Morales 2000 & 4 & 233 & 6 & 281 & $4.4 \%$ \\
\hline Oteiza 2004 & 1 & 124 & 0 & 123 & $0.7 \%$ \\
\hline Perez 2005 & 3 & 180 & 6 & 180 & $3.7 \%$ \\
\hline Tzovaras 2007 & 5 & 190 & 9 & 189 & $6.0 \%$ \\
\hline Subtotal (95\% CI) & & 1526 & & 1574 & $28.4 \%$ \\
\hline Total events: & 26 & & 38 & & \\
\hline
\end{tabular}

Heterogeneity: $\mathrm{Tau}^{2}=0.00 ; \mathrm{Chi}^{2}=2.45, \mathrm{df}=8(\mathrm{P}=0.96) ; \mathrm{I}^{2}=0 \%$

Test for overall effect: $\mathrm{Z}=1.33(\mathrm{P}=0.18)$

2.2.2 high infection risk environment

$\begin{array}{lrrrrr}\text { Al-Fatah 2011 } & 3 & 100 & 5 & 100 & 3.5 \% \\ \text { Celdran 2004 } & 0 & 50 & 4 & 49 & 0.8 \% \\ \text { Ergul 2011 } & 5 & 100 & 7 & 100 & 5.6 \% \\ \text { Ijaz 2010 } & 2 & 50 & 5 & 50 & 2.7 \% \\ \text { Mazaki 2013 } & 2 & 100 & 13 & 100 & 3.2 \% \\ \text { Othman 2011 } & 3 & 50 & 5 & 48 & 3.7 \% \\ \text { Rahmani 2012 } & 4 & 141 & 8 & 141 & 5.0 \% \\ \text { Shankar 2010 } & 11 & 172 & 16 & 162 & 12.8 \% \\ \text { Thakur 2010 } & 3 & 29 & 3 & 26 & 3.0 \% \\ \text { Ullah 2013 } & 6 & 83 & 15 & 83 & 8.6 \% \\ \text { Wang 2013 } & 29 & 768 & 19 & 392 & 21.7 \% \\ \text { Yerdel 2001 } & 0 & 136 & 9 & 133 & 0.9 \% \\ \text { Subtotal (95\% CI) } & & \mathbf{1 7 7 9} & & \mathbf{1 3 8 4} & \mathbf{7 1 . 6 \%} \\ \text { Total events: } & 68 & & 109 & & \end{array}$

$0.60[0.15,2.44]$

$0.11[0.01,1.97]$

$0.71[0.23,2.18]$

$0.40[0.08,1.97]$

$0.15[0.04,0.66]$

$0.58[0.15,2.28]$

$0.50[0.15,1.62]$

$0.65[0.31,1.35]$

$0.90[0.20,4.06]$

$0.40[0.16,0.98]$

$0.78[0.44,1.37]$

$0.05[0.00,0.88]$

$0.56[0.41,0.77]$

Total events:

109

Heterogeneity: $\mathrm{Tau}^{2}=0.00 ; \mathrm{Chi}^{2}=10.18, \mathrm{df}=11(\mathrm{P}=0.51) ; \mathrm{I}^{2}=0 \%$

Test for overall effect: $\mathrm{Z}=3.64(\mathrm{P}=0.0003)$

Total $(95 \%$ CI $)$

Total events: 94 147

Heterogeneity: $\mathrm{Tau}^{2}=0.00 ; \mathrm{Chi}^{2}=13.17, \mathrm{df}=20(\mathrm{P}=0.87) ; \mathrm{I}^{2}=0 \%$

Test for overall effect: $\mathrm{Z}=3.79(\mathrm{P}=0.0002)$

Test for subgroup differences: $\mathrm{Chi}^{2}=0.66, \mathrm{df}=1(\mathrm{P}=0.42), \mathrm{I}^{2}=0 \%$
Risk Ratio

M-H, Random, 95\% CI

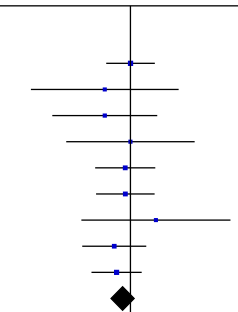

$0.60[0.46,0.78]$

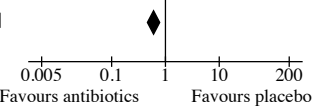


Analysis 2.3. Comparison 2: Wound infections hernioplasty, Outcome 3: Deep Surgical Site Infections (DSSI)

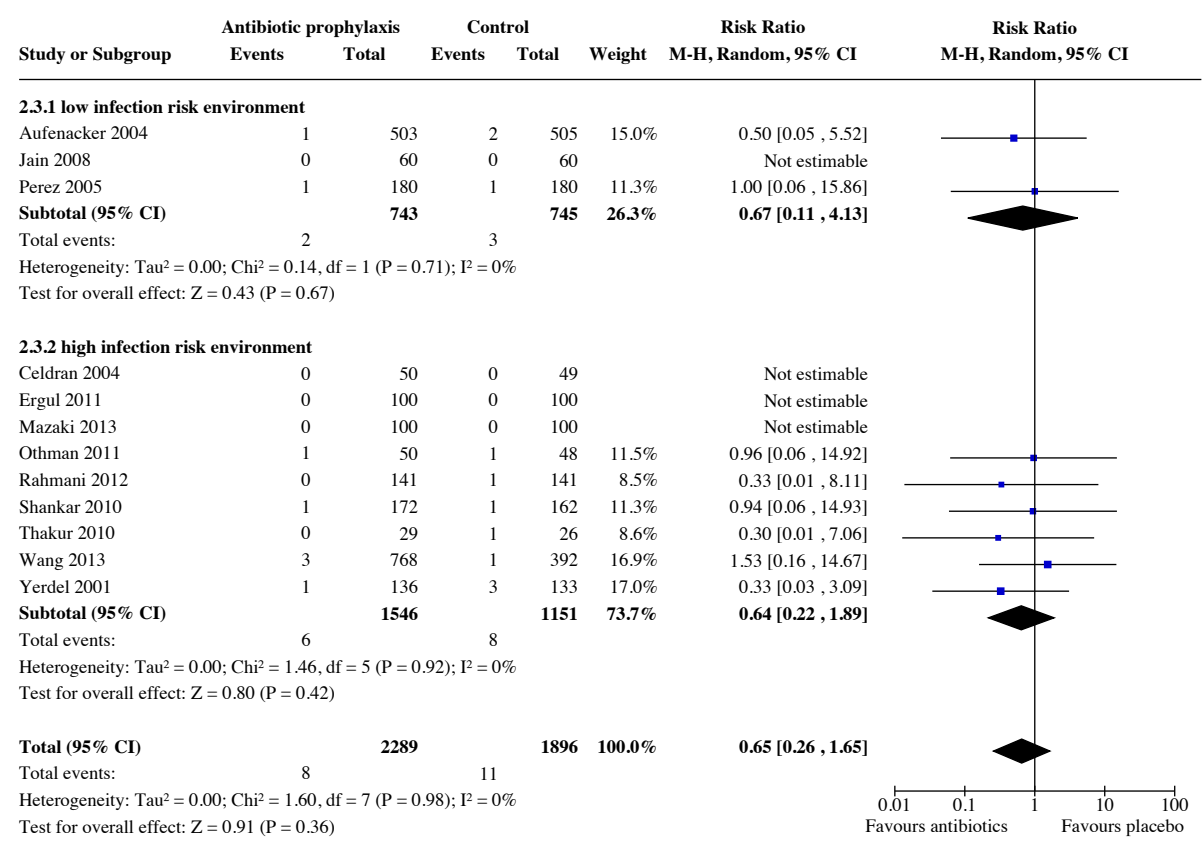


ADDITIONAL TABLES

Table 1. Overview of treatment characteristics of the included herniorrhaphy studies

\begin{tabular}{|c|c|c|c|c|c|c|c|}
\hline & Antibiotic & Dose (g) & Administration route & $\begin{array}{l}\text { Follow-up pe- } \\
\text { riod }\end{array}$ & $\begin{array}{l}\text { Operative time (min- } \\
\text { utes); (mean } \pm \text { SD or me- } \\
\text { dian (range)) }\end{array}$ & $\begin{array}{l}\text { Infection risk environ- } \\
\text { ment } \\
\text { (Infection percentage } \\
\text { in control group) }\end{array}$ & Remark \\
\hline $\begin{array}{l}\text { Andersen } \\
1980\end{array}$ & AMP & 1 & in fascia & $\begin{array}{l}1 \mathrm{~m}, 3 \mathrm{~m}, 6 \mathrm{~m} \\
12 \mathrm{~m}\end{array}$ & not reported & Low $(4.0 \%)$ & $\begin{array}{l}\text { Several surgery } \\
\text { types performed }\end{array}$ \\
\hline Evans 1973 & CLR & $1(3 \times)$ & $\begin{array}{l}\text { intravenous }(1 \mathrm{x}) / \text { intra- } \\
\text { muscular }(2 \mathrm{x})\end{array}$ & $1 \mathrm{~m}$ & not reported & Low $(4.1 \%)$ & $\begin{array}{l}\text { Several surgery } \\
\text { types performed }\end{array}$ \\
\hline $\begin{array}{l}\text { Lazorthes } \\
1992\end{array}$ & CAM & 0.75 & $\begin{array}{l}\text { subcutaneous (added to } \\
\text { local anaesthesia) }\end{array}$ & $1 \mathrm{~m}$ & not reported & Low $(4.6 \%)$ & \\
\hline Platt 1990 & CON & 1 & intravenous & $1 \mathrm{w}, 4$ to $6 \mathrm{w}$ & PG: $75 \pm 32$ / CG: $75 \pm 30$ & Low $(1.9 \%)$ & $\begin{array}{l}\text { Several surgery } \\
\text { types performed }\end{array}$ \\
\hline Taylor 1997 & AMC & 1.2 & intravenous & 4 to $6 \mathrm{w}$ & not reported & High (8.9\%) & \\
\hline
\end{tabular}

$P G=$ prophylactic group, $C G=$ control group

AMC = Amoxicillin-clavoulanic acid, AMP = ampicillin, CAM = cefamandole, CON = cefonicid, CLR = cephaloridine (Abbreviations according to EUCAST System for Antimicrobial Abbreviations)

$w=$ week, $m=$ month, $y=$ year

Infection risk environment $(\mathrm{L}=$ low; $\leq 5 \%$ infections in control group for all wound infections (SSSI $+\mathrm{DSSI}), \mathrm{H}=$ high; $>5 \%$ infections in control group for all wound infections $(\mathrm{SSSI}+\mathrm{DSSI}))$. 
Table 2. Overview of treatment characteristics of the included hernioplasty studies

\begin{tabular}{|c|c|c|c|c|c|c|}
\hline & $\begin{array}{l}\text { An- } \\
\text { tibiot- } \\
\text { ic }\end{array}$ & $\begin{array}{l}\text { Dose } \\
\text { (g) }\end{array}$ & $\begin{array}{l}\text { Administra- } \\
\text { tion route }\end{array}$ & $\begin{array}{l}\text { Follow-up } \\
\text { period }\end{array}$ & $\begin{array}{l}\text { Operative time (minutes); (mean } \pm \text { SD or medi- } \\
\text { an (range)) }\end{array}$ & $\begin{array}{l}\text { Infection risk } \\
\text { environment } \\
\text { (Infection per- } \\
\text { centage in con- } \\
\text { trol group) }\end{array}$ \\
\hline $\begin{array}{l}\text { Al-Fatah } \\
2011\end{array}$ & AMC & 1.2 & intravenous & $1 \mathrm{w} / 1 \mathrm{~m}$ & PG: 45 (20 to 90$) ; C G: 45$ (20 to 80 ) & High $(5.0 \%)$ \\
\hline $\begin{array}{l}\text { Aufenacker } \\
2004\end{array}$ & CUR & 1.5 & intravenous & $1 \mathrm{w}, 2 \mathrm{w}, 3 \mathrm{~m}$ & PG: 40 (IQR 30 to 50); CG 40 (IQR 28 to 51 ) & Low $(1.8 \%)$ \\
\hline $\begin{array}{l}\text { Bidhur } \\
2013\end{array}$ & CUR & 1.5 & intravenous & $1 \mathrm{w}, 1 \mathrm{~m}$ & $\begin{array}{l}51.3 \pm 9.8 \text { (range } 40 \text { to } 75 \text { min) (overall popula- } \\
\text { tion) } / \text { Time }<50 \text { min: } P G: n=19(63 \%) ; C G: n=15 \\
(50 \%)\end{array}$ & Low $(3.3 \%)$ \\
\hline $\begin{array}{l}\text { Celdran } \\
2004\end{array}$ & $\mathrm{CZO}$ & 1 & not reported & $\begin{array}{l}1 \mathrm{w}, 1 \mathrm{~m}, 3 \\
\mathrm{~m}, 6 \mathrm{~m}, 1 \mathrm{y} \\
2 \mathrm{y}\end{array}$ & $P G: 65 \pm 23 ; C G: 64 \pm 14$ & High $(8.2 \%)$ \\
\hline Ergul 2011 & $\mathrm{CZO}$ & 1 & intravenous & 1 to $6 \mathrm{~d}, 1 \mathrm{~m}$ & PG: 60 (35 to 160 ); CG: 60 (40 to 135 ) & High $(7.0 \%)$ \\
\hline Goyal 2011 & AMC & 1.2 & intravenous & $1 \mathrm{w}$ & not reported & Low $(3.0 \%)$ \\
\hline ljaz 2010 & $\mathrm{CZO}$ & 1 & intravenous & $1 \mathrm{w}, 2 \mathrm{w}, 1 \mathrm{~m}$ & not reported & High $(10.0 \%)$ \\
\hline Jain 2008 & AMC & 1.2 & intravenous & $\begin{array}{l}1 \mathrm{w}, 2 \mathrm{w}, 1 \mathrm{~m} \\
1 \mathrm{y}\end{array}$ & PG: $56.33 \pm 11.67 ; C G: 60.33 \pm 6.81$ & Low $(1.7 \%)$ \\
\hline $\begin{array}{l}\text { Kochhar } \\
2014\end{array}$ & AMC & 1.2 & intravenous & $1 \mathrm{w}, 1 \mathrm{~m}$ & not reported & Low $(4.7 \%)$ \\
\hline $\begin{array}{l}\text { Mazaki } \\
2013\end{array}$ & $\mathrm{CZO}$ & 1 & intravenous & $1 \mathrm{w}, 1 \mathrm{~m}, 3 \mathrm{~m}$ & PG: $66.3 \pm 25.4 ;$ CG: $65.2 \pm 27.1$ & High (13.0\%) \\
\hline $\begin{array}{l}\text { Morales } \\
2000\end{array}$ & $\begin{array}{l}\text { CZO } \\
\text { (or } \\
\text { ERY) }\end{array}$ & $\begin{array}{l}2 \text { (or } \\
1)\end{array}$ & intravenous & $1 \mathrm{w}, 1 \mathrm{~m}, 1 \mathrm{y}$ & not reported & $\operatorname{Low}(2.1 \%)$ \\
\hline Oteiza 2004 & AMC & 2 & intravenous & $1 \mathrm{w}, 1 \mathrm{~m}$ & PG and CG: Mean 40 min & $\operatorname{Low}(0 \%)$ \\
\hline $\begin{array}{l}\text { Othman } \\
2011\end{array}$ & AMC & 1.2 & intravenous & $1 \mathrm{w}, 1 \mathrm{~m}$ & PG $38.8 \pm 10.8 ;$ CG $40.9 \pm 11.1$ & High $(12,5 \%)$ \\
\hline Perez 2005 & $\mathrm{CZO}$ & 1 & intravenous & $1 \mathrm{w}, 2 \mathrm{w}, 1 \mathrm{~m}$ & PG 52.18 $\pm 16.4 ;$ CG $54.07 \pm 15.3$ & Low $(3.9 \%)$ \\
\hline $\begin{array}{l}\text { Rahmani } \\
2012\end{array}$ & CLT & 1 & intravenous & $12 \mathrm{w}$ & not reported & High $(6.4 \%)$ \\
\hline $\begin{array}{l}\text { Razack } \\
2015\end{array}$ & $\mathrm{CZO}$ & 1 & intravenous & $1 \mathrm{w}, 1 \mathrm{~m}$ & PG: $53.54 \pm 15.82 ;$ CG $52.60 \pm 15.28$ & High $(9.3 \%)$ \\
\hline $\begin{array}{l}\text { Shankar } \\
2010\end{array}$ & $\mathrm{CZO}$ & 1 & intravenous & $1 \mathrm{w}, 1 \mathrm{~m}$ & PG $53.54 \pm 15.82 ;$ CG $52.60 \pm 15.28$ & High $(10.5 \%)$ \\
\hline
\end{tabular}


Table 2. Overview of treatment characteristics of the included hernioplasty studies (Continued)

\begin{tabular}{|c|c|c|c|c|c|c|}
\hline $\begin{array}{l}\text { Thakur } \\
2010\end{array}$ & $\mathrm{CZO}$ & 1.5 & intravenous & $1 \mathrm{w}, 1 \mathrm{~m}$ & $\begin{array}{l}\text { PG: } 79.3 \%(n=23) \text { had the total duration of } \\
\text { surgery }>1 \text { hour; CG: } 92.3 \%(n=24) \text { had the to- } \\
\text { tal duration of surgery }>1 \text { hour. }\end{array}$ & High (15.4\%) \\
\hline $\begin{array}{l}\text { Tzovaras } \\
2007\end{array}$ & AML & 1.2 & intravenous & $1 \mathrm{w}, 1 \mathrm{~m}$ & PG: 45 (20 to 90$) ; C G: 45$ (20 to 80 ) & Low $(4.8 \%)$ \\
\hline Ullah 2013 & AMC & 1 & not reported & $2 \mathrm{~d}, 2 \mathrm{w}$ & not reported & High $(18.1 \%)$ \\
\hline Wang 2013 & $\begin{array}{l}\text { CZO } \\
\text { or LEV }\end{array}$ & 1 or & intravenous & $\begin{array}{l}1 \mathrm{w}, 2 \mathrm{w}, 3 \mathrm{w}, \\
1 \mathrm{~m}\end{array}$ & not reported & High (5.1\%) \\
\hline Yerdel 2001 & AMS & 1.5 & intravenous & $\begin{array}{l}1 \mathrm{w}, 4 \text { to } 6 \mathrm{w}, \\
6 \mathrm{~m}, 1 \mathrm{y}\end{array}$ & PG: $64.18 \pm 22.8 ; C G: 62.78 \pm 19.3$ & High $(9.0 \%)$ \\
\hline
\end{tabular}

$P G=$ prophylactic group, $C G=$ control group

$A M C=$ Amoxicillin-clavoulanic acid, $A M L=$ ampicillin-clavulanic acid, AMS = ampicillin-sulbactam, $C L T=$ cephalotin, $C U R=$ cefuroxime, $C Z O$ = cefazolin, ERY = erythromycin, LEV = Levofloxacin (Abbreviations according to EUCAST System for Antimicrobial Abbreviations) $\mathrm{w}=$ week, $\mathrm{m}=$ month, $\mathrm{y}=$ year

Infection risk environment $(\mathrm{L}=$ low; $\leq 5 \%$ infections in control group for all wound infections (SSSI $+\mathrm{DSSI}), \mathrm{H}=$ high; > 5\% infections in control group for all wound infections (SSSI + DSSI)).

Table 3. Overview of participant characteristics of the included herniorrhaphy studies

\begin{tabular}{|c|c|c|c|c|c|c|}
\hline & $\%$ male & Age (years) in mean \pm SD or median (range) & $\begin{array}{l}\text { Partici- } \\
\text { pants } \\
\text { age< } \\
18 \mathrm{y} \\
\text { includ- } \\
\text { ed }\end{array}$ & $\begin{array}{l}\text { Inguinal } \\
\text { (I) or } \\
\text { femoral } \\
\text { (F) } \\
\text { hernia }\end{array}$ & ASA class & $\begin{array}{l}\text { BMI mean } \pm \text { SD or } \\
\text { median (range) }\end{array}$ \\
\hline $\begin{array}{l}\text { Ander- } \\
\text { sen } 1980\end{array}$ & $\begin{array}{l}\text { PG: } 72 \\
\text { CG: } 73\end{array}$ & $\begin{array}{l}\text { PG: } 54 \text { to } 56 \text { years (range } 21 \text { to } 82 \text { ); } \\
\text { CG: } 55 \text { years (range } 20 \text { to } 82 \text { ) }\end{array}$ & No & $\mathrm{I}+\mathrm{F}$ & $\begin{array}{l}\text { Not report- } \\
\text { ed }\end{array}$ & Not reported \\
\hline $\begin{array}{l}\text { Evans } \\
1973\end{array}$ & $\begin{array}{l}\text { not report- } \\
\text { ed }\end{array}$ & not reported & $\begin{array}{l}\text { not re- } \\
\text { ported }\end{array}$ & $\mathrm{I}+\mathrm{F}$ & $\begin{array}{l}\text { Not report- } \\
\text { ed }\end{array}$ & Not reported \\
\hline $\begin{array}{l}\text { La- } \\
\text { zorthes } \\
1992\end{array}$ & $\begin{array}{l}\text { PG: } 90 ; \text { CG: } \\
88\end{array}$ & PG: 62 (11 to 90$) /$ CG: 70 (16 to 92 ) & Yes & $\mathrm{I}+\mathrm{F}$ & $\begin{array}{l}\text { Not report- } \\
\text { ed }\end{array}$ & Not reported \\
\hline $\begin{array}{l}\text { Platt } \\
1990\end{array}$ & $\begin{array}{l}\text { PG: 91; CG: } \\
89\end{array}$ & PG: $51.0 \pm 17.0 /$ CG: $49.8 \pm 17.6$ & No & $\mathrm{I}+\mathrm{F}$ & $\begin{array}{l}\text { Not report- } \\
\text { ed }\end{array}$ & $\begin{array}{l}P G 24.6 \pm 3.2 ; C G \\
24.7 \pm 3.2\end{array}$ \\
\hline $\begin{array}{l}\text { Taylor } \\
1997\end{array}$ & $\begin{array}{l}\text { PG: 95; CG: } \\
95\end{array}$ & PG: $56.7 \pm 17.4$ / CG: $56.6 \pm 16.5$ & No & $\mathrm{I}+\mathrm{F}$ & $\begin{array}{l}\text { Not report- } \\
\text { ed }\end{array}$ & Not reported \\
\hline
\end{tabular}

$P G=$ prophylactic group,$C G=$ control group 
Table 4. Overview of participant characteristics of the included hernioplasty studies

\begin{tabular}{|c|c|c|c|c|c|c|}
\hline & $\%$ male & Age (years) in mean $\pm \mathrm{SD}$ or median (range) & $\begin{array}{l}\text { Par- } \\
\text { tici- } \\
\text { pants } \\
\text { age< } \\
18 y \\
\text { in- } \\
\text { clud- } \\
\text { ed }\end{array}$ & $\begin{array}{l}\text { In- } \\
\text { guinal } \\
\text { (I) or } \\
\text { femoral } \\
\text { (F) } \\
\text { hernia }\end{array}$ & $\begin{array}{l}\text { ASA } \\
\text { class }\end{array}$ & $\begin{array}{l}\text { BMI mean } \pm \text { SD or } \\
\text { median (range) }\end{array}$ \\
\hline $\begin{array}{l}\text { Al-Fatah } \\
2011\end{array}$ & PG: 94; CG: 96 & PG: 63 (17 to 87 ); CG: 63 (15 to 90 ) & Yes & 1 & I; II; III & $\begin{array}{l}\text { PG: } 26 \text { (18 to } 34) ; C G: \\
26 \text { ( } 20 \text { to } 33 \text { ) }\end{array}$ \\
\hline $\begin{array}{l}\text { Aufenack- } \\
\text { er } 2004\end{array}$ & PG: 96; CG: 97 & PG: $58.3 \pm 12.9$; CG: $58.2 \pm 13.2$ & No & 1 & $\begin{array}{l}\text { Not re- } \\
\text { ported }\end{array}$ & Not reported \\
\hline $\begin{array}{l}\text { Bidhur } \\
2013\end{array}$ & PG: 100; CG: 97 & $\begin{array}{l}38.5 \pm 17.7 \text { (range: } 19 \text { to } 90 \text { years) } \\
\text { (overall study population) }\end{array}$ & No & 1 & $\begin{array}{l}\text { Not re- } \\
\text { ported }\end{array}$ & Not reported \\
\hline $\begin{array}{l}\text { Celdran } \\
2004\end{array}$ & PG: 94; CG: 86 & PG: $58 \pm 13 ; C G: 58 \pm 17$ & No & I & ।; II & $\begin{array}{l}\text { PG: } 26.1 \pm 5 / \text { CG: } 26.2 \\
\pm 5\end{array}$ \\
\hline Ergul 2011 & PG: 95; CG: 89 & PG: $48 \pm 17 ; C G: 50 \pm 15$ & No & I & I; II; III; IV & Not reported \\
\hline $\begin{array}{l}\text { Goyal } \\
2011\end{array}$ & NR & range 11 to 90 (overall study population) & Yes & 1 & $\begin{array}{l}\text { Not re- } \\
\text { ported }\end{array}$ & Not reported \\
\hline ljaz 2010 & 99 (overall) & PG: 44.06; CG: 44.84 & No & 1 & $\begin{array}{l}\text { Not re- } \\
\text { ported }\end{array}$ & Not reported \\
\hline Jain 2008 & PG: $100 ;$ CG:100 & PG: $41.28 \pm 11.49 ;$ CG: $40.2 \pm 9.84$ & No & I & $1 / 11$ & Not reported \\
\hline $\begin{array}{l}\text { Kochhar } \\
2014\end{array}$ & 96 (overall) & PG: $37.42 \pm 9.9 ;$ CG: $37.42 \pm 11.5$ & Yes & 1 & $\begin{array}{l}\text { Not re- } \\
\text { ported }\end{array}$ & Not reported \\
\hline $\begin{array}{l}\text { Mazaki } \\
2013\end{array}$ & PG: 89/ CG: 94 & PG: 69 (57 to 76 ); CG: 72 (60 to 77 ) & No & I & I; II; III & $\begin{array}{l}P G: 23.0 \pm 2.8 P G ; C G: \\
22.7 \pm 3.0\end{array}$ \\
\hline $\begin{array}{l}\text { Morales } \\
2000\end{array}$ & 89 (overall) & 54.2 (17 to 87 ) (overall study population) & No & $1+F$ & I; II; III & Not reported \\
\hline $\begin{array}{l}\text { Oteiza } \\
2004\end{array}$ & PG: 89; CG: 82 & PG: 58 (22 to 91$) /$ CG: 56.2 (17 to 88 ) & Yes & $\mathrm{I}+\mathrm{F}$ & I; II; III & Not reported \\
\hline $\begin{array}{l}\text { Othman } \\
2011\end{array}$ & PG: 96; CG: 100 & PG: $43.4 \pm 19.8 /$ CG: $44.5 \pm 20.5$ & No & I & $\begin{array}{l}\text { Not re- } \\
\text { ported }\end{array}$ & Not reported \\
\hline $\begin{array}{l}\text { Perez } \\
2005\end{array}$ & PG: 98; CG: 98 & PG: $61.37 \pm 13.2 /$ CG: $60.8 \pm 14.5$ & No & I & ।; II & Not reported \\
\hline $\begin{array}{l}\text { Rahmani } \\
2012\end{array}$ & PG: 45; CG: 41 & 25 to 84 (overall population) & No & 1 & $\begin{array}{l}\text { not re- } \\
\text { ported }\end{array}$ & Not reported \\
\hline $\begin{array}{l}\text { Razack } \\
2015\end{array}$ & PG: 99; CG: 100 & PG: $42.44 \pm 15.61$ / CG: $45.56 \pm 15.43$ & Yes & 1 & ।; II & Not reported \\
\hline
\end{tabular}


Table 4. Overview of participant characteristics of the included hernioplasty studies (Continued)

\begin{tabular}{|c|c|c|c|c|c|c|}
\hline $\begin{array}{l}\text { Shankar } \\
2010\end{array}$ & PG: 99; CG: 98 & PG: $44.44 \pm 15.59$ / CG: $45.56 \pm 16.43$ & Yes & 1 & I; II & Not reported \\
\hline $\begin{array}{l}\text { Thakur } \\
2010\end{array}$ & PG: 100; CG: 100 & Not reported & No & 1 & $\begin{array}{l}\text { Not re- } \\
\text { ported }\end{array}$ & Not reported \\
\hline $\begin{array}{l}\text { Tzovaras } \\
2007\end{array}$ & PG: 94; CG: 94 & PG: 63 (17 to 87$)$ / CG: 63 (15 to 90) & Yes & 1 & I; II; III & $\begin{array}{l}\text { PG: } 26 \text { (18 to } 34) \text {; CG: } \\
26 \text { ( } 20 \text { to } 33 \text { ) }\end{array}$ \\
\hline Ullah 2013 & PG: 100; CG: 100 & PG: $54.33 \pm 11.77$ / CG: $52.58 \pm 11.80$ & No & I & $\begin{array}{l}\text { Not re- } \\
\text { ported }\end{array}$ & Not reported \\
\hline $\begin{array}{l}\text { Wang } \\
2013\end{array}$ & $\begin{array}{l}\text { PG group 1: 90; } \\
\text { PG group 2: 91; } \\
\text { CG: } 90\end{array}$ & $\begin{array}{l}\text { PG group } 1: 53 \pm 16.9 ; \text { PG group } 2: 54.6 \pm \\
16.2 / \\
\text { CG: } 56 \pm 17.3\end{array}$ & Yes & I & $\mathrm{I}, \geq \mathrm{I}$ & 18 to 32 (overall) \\
\hline $\begin{array}{l}\text { Yerdel } \\
2001\end{array}$ & PG: 90; CG: 95 & PG: $55.57 \pm 15.1$; CG: $55.78 \pm 13.8$ & No & 1 & I; II & $\begin{array}{l}P G: 24.95 \pm 2.6 ; C G: \\
25.02 \pm 3.0\end{array}$ \\
\hline
\end{tabular}

$P G=$ prophylactic group, $C G=$ control group 


\title{
Summarizing discussion, conclusions and future perspectives
}

\begin{abstract}
An inguinal hernia repair is the most frequently performed surgical procedure worldwide. In the past decades research has led to an impressive increase in the quality of inguinal hernia repair. Although the search for further technical improvement remains on-going, attention is shifting towards improving the efficiency of inguinal hernia care. However, what does efficiency embody and how can it be ensured by health care organisations?
\end{abstract}

The Institute of Medicine (IOM) defines efficiency as "avoiding waste, including waste of equipment, supplies, ideas, and energy". Waste regarding the organization of healthcare is a multidimensional problem that can occur in a variety of areas (e.g. unnecessary services, inefficient distribution of care, failures of preventive medicine or excess administrative costs). In order to avoid waste and increase efficiency, first each process in the chain of care has to be mapped out. Once the clearly inefficient steps are identified, efforts should be made to eliminate or adjust these steps in order to reduce potential harm, excess costs or input of time. Although this approach is ostensibly simple it remains difficult to identify the inefficient steps. After all, how do we measure efficiency?

The analysis and measurement of efficiency in healthcare is a complex undertaking, not in the least due to the various objectives and multiple inputs and outputs. It is often necessary to look at multiple levels and take the numerous stakeholders into account. ${ }^{2,3}$ These stakeholders include patients, doctors, the hospital management, health insurance companies and politicians. Despite its importance, there is no clear definition or measure of efficiency. Hence, with regard to specific treatments, effectiveness is generally inferred from money-related measures such as hospital costs, length of stay, the number of outpatients visits and work absenteeism. ${ }^{4}$ Societies, governments and hospitals would benefit from a universal measure for healthcare efficiency, if only to identify the inefficient pathways.

Inefficient use of healthcare is raising concerns for a number of reasons. Firstly, patients that do not receive the best possible care may be deprived of potential health gain or even harmed by redundant medical interventions. Secondly, an inefficient organization of healthcare and thus excess costs may deny funding and resources to other medical or paramedical authorities. Moreover, healthcare efficiency could possibly result in the reallocation of healthcare expenditure to other sections of society (eg. education, nutrition, or the environment). ${ }^{5}$ And lastly, inefficient healthcare might eventually reduce the willingness of society to address the costs. 
Concerning the question of how health care systems can further ensure efficiency in inguinal hernia surgery, it would be wrong to pretend there is one answer. 'Efficiency' is a dynamic concept that will continue to evolve. However, the work presented in this thesis is a modest step towards (evaluating) efficiency in the current practice of inguinal hernia repair.

\section{Simplification of care}

Efficiency can be improved by simplifying care and avoiding unnecessary hospital visits. One way of achieving this is by streamlining and speeding up the surgical treatment pathway. Combining the traditionally separate preoperative evaluation, diagnostics and subsequent operation into one single visit for elective surgery of straightforward surgical diagnosis is an effective adjustment. Single visit surgery has received increased attention over the last decades, demonstrating promising results in several low complex surgical specialties (e.g. ophthalmic operation, hand surgery and treatment for basal cell carcinoma). ${ }^{6-8}$ Feasibility and safety of day-case surgery for laparoscopic cholecystectomy has already been described in 1993. ${ }^{9}$ Likewise, for hernia surgery, the single-visit pathway has proved to be efficient and safe, with high attendance rates and a high level of patient satisfaction.10,11 Despite the strong intuitive appeal, studies on the impact of single visit hernia surgery on reducing total health care costs have never been published. In Chapter 2 the outcomes of a cost-comparison between the single-visit and the regular TEP pathway are presented from both a hospital- and societal point of view. The results demonstrate that the single-visit endoscopic inguinal hernia repair outprices the regular TEP inguinal hernia repair, rendering it the preferred alternative. In an employed healthy population, single-visit TEP inguinal hernia repair results in a $20 \%$ decrease in costs, of which the majority is determined by a reduction in societal costs. The hospital cost reduction was relatively small and mainly achieved by the difference in pre-operative screening (POS) of a regular (in hospital POS) and a single-visit (telephone/electronic POS) procedure. However, the societal costs of a single-visit procedure dropped by one-fourth compared to the regular care. This was predominantly a result of fewer lost workdays and hospital visits, with the associated reduction of travel expenses. Since employers and employees will benefit most from adjusting in-hospital patient routings, it is worthwhile for hospitals to re-evaluate all potentially ingrained in-hospital routings. Considering implementation of the single-visit routing for other commonly performed elective surgical procedures (e.g. cholecystectomy, colorectal procedures or varicose veins) and consequently reduce societal costs, might further drive down healthcare expenditures.

\section{Prevention of medical overuse}

Medical overuse is defined as "a health care service that is provided under circumstances in which its potential for harm exceeds the possible benefit". ${ }^{2}$ Since quantifying benefit and harm often proves difficult, the term "overuse" is often referred to as any service that is unnecessary in any way and for any reason. ${ }^{13}$ In case of inguinal hernias, this applies particularly to medical imaging. 
An apparent inguinal hernia, with evident clinical features, does not require further imaging tests. Nevertheless, chapter 3 clearly points out that ultrasound (US) diagnostics in patients with clinically apparent inguinal hernias is redundantly requested in primary care. Approximately a quarter of newly referred patients with suspicion of an inguinal hernia to the surgical outpatient department underwent ultrasound in advance. Since nearly $80 \%$ of these patients showed an evident inguinal hernia on physical examination (PE), the majority of groin ultrasound could have been avoided.

Obviously, it cannot be expected that the PE by general practitioners (GP) is of the same level as that by surgeons working in a high-volume hernia centre. However, requesting groin US for confirmation of a strong clinical suspicion is not indicated. Furthermore, requesting groin US out of fear of missing the diagnosis of an inguinal hernia that will become incarcerated is unnecessary since the risk is as low as under $2.5 \%$ annually. ${ }^{14}$ The training of GPs in performing $\mathrm{PE}$, and raising awareness of redundantly requested groin US and the low risk of incarceration, might reduce requested US, lower costs and increase efficiency. Radiology departments often enable GPs to refer patients for US directly without prior referral to secondary care. In case of a high suspicion of an inguinal hernia it might be more efficient to directly refer to the surgical outpatient clinic.

The overuse of ultrasound is also frequently seen with recurrent inguinal hernias. Although the majority of recurrent hernias present with clinical features that are similar to those of primary inguinal hernias, groin US is frequently requested by both GPs and surgeons. Chapter 6 describes that almost two-third of patients re-operated for suspicion of a recurrent hernia after a primary TEP underwent US in advance, of which most were requested by the surgeon. Since a positive PE performed by a surgeon matched the intra-operative findings in $97 \%$ of cases, medical imaging does not necessarily have to be incorporated in the diagnostic workup of a recurrent inguinal hernia. Only in the work-up of a dubious recurrence with complaints ultrasound may be of value. Again, directly referring patients to the surgical outpatient clinic, instead of performing US in the primary care setting, might be more efficient.

Another example of overuse is the excessive use of antibiotics. Though it would be hard to undermine its importance in treating bacterial infections, preventing disease from spreading and reducing complications, the crisis of antibiotic resistance is worsening.$^{15}$ Regarding hernia repair, laparoscopic techniques are sterile surgical procedures with low rates of postoperative wound infections. Hence, antibiotic prophylaxis is not recommended. ${ }^{16}$ Whether to use antibiotic prophylaxis in open hernia repair remains a matter of discussion. Chapter 8 describes a systematic review of all available evidence from RCTs comparing antibiotic prophylaxis versus a placebo or no prophylaxis for open inguinal and/or femoral hernia repair. The results demonstrate that administration of antibiotic prophylaxis for elective open inguinal or femoral hernia repair cannot be universally recommended. Only in a high infection risk environment, antibiotic prophylaxis might reduce superficial wound infections. However, in a low infection risk environment, antibiotic prophylaxis has little or no effect on the reduction of superficial wound infections. Regardless of the environment, the prevention of deep wound infections by antibiotic prophylaxis could neither be confirmed nor refuted in comparison with a placebo (or no treatment). Hence, in the majority of European countries, administration of antibiotic prophylaxis in open inguinal hernia repair can be considered overtreatment. 
A frequently used term relating to overuse is "overdiagnosing", which is defined as "identification of abnormalities that are indolent, non-progressive, or regressive and that will not cause symptoms or shorten an individual his life if left untreated". ${ }^{7}$ Regarding inguinal hernias, overdiagnosing is most likely to happen in case of ambiguous groin complaints. The need for imaging is quite understandable in scenarios where groin complaints are not accompanied by evident clinical features. Furthermore, US undoubtedly has a place in the work-up of ambiguous groin complaints. However, it is questionable whether all inguinal hernias diagnosed on ultrasound are truly causing the complaints. This especially applies to subclinical inguinal hernias diagnosed solely on US. Chapter 4 describes the results of a cross-sectional study assessing the prevalence of an unknown inguinal hernia among healthy, working-age men without groin complaints. In 16\% of men between 40 and 67 years old without groin complaints or a medical history of an inguinal hernia or groin surgery, an inguinal hernia was found on groin US. In half of these men PE confirmed an inguinal hernia. Taking into account the widespread use of groin US, the high prevalence poses a considerable risk of coincidentally detected inguinal hernias that may wrongly be considered as the cause of groin pain and may prompt superfluous surgical interventions. A scenario in which the potential harm exceeds the benefits. Therefore, in case of groin complaints without evident clinical features, when requesting groin US both surgeons and GPS should be aware of the risk of overdiagnosing. It is important to realise the broad differential diagnosis of groin pain and first rule out other causes.

\section{Reduction of adverse events}

As a results-driven organisation of healthcare is becoming increasingly important in the pursuit of high-value medical practice, another way to achieve efficiency is through reducing adverse events. ${ }^{18}$ Regarding inguinal hernia repair, chronic postoperative inguinal pain (CPIP) and recurrences can be considered the most commonly reported adverse event. ${ }^{16}$

Although recurrence rates have fallen below 5.0\% with the introduction of mesh repair, recurrences after TEP inguinal hernia repair remain a clinical problem. Further preventing recurrences would increase efficiency and lower the costs. Therefore, identifying and addressing the underlying causes of a recurrent hernia is of interest. Given the generally low recurrence rates, this poses a challenge. A high volume of patients is required to perform research into recurrences, especially with the aim of identifying risk factors for the development of recurrences. However, since inguinal hernia repair is one of the most commonly performed surgical procedures, small improvements can lead to significant health care enhancements. In order to understand the aetiology of recurrences after TEP-repair, an 11-year analysis of all re-operated groins for recurrence-like symptoms in the high-volume Dutch Hernia Centre was performed, as presented in Chapter 5. Since all surgeons had extensive experience, elimination of the learning curve-related recurrences was justifiable. Three-fourth of all recurrences involved a direct hernia after a primary direct hernia repair. In nearly one-fifth the recurrent hernia appeared to be a lipoma clinically mimicking a 'true' recurrent hernia. These lipomas might have been formed after the operation. However, there is a distinct possibility that these lipomas were present and overseen during the initial repair. Furthermore, this 
analysis demonstrates that in patients in whom a primary large defect was reported, hernias recurred as their initial type, especially when the mesh was not fixated. Moreover, patients undergoing bilateral TEP hernia repair were more prone to develop a recurrence. Hence, in the light of the efficiency of inguinal hernia repair, mesh fixation should be considered in large direct and bilateral hernia defects. It is important to thoroughly inspect the complete inguinal floor and inguinal canal in order to adequately remove lipomas. The advice is to treat alleged recurrent inguinal hernias with an anterior approach. Using this approach, 'true' inguinal hernia recurrences are treated according the international guidelines ${ }^{16}$ and isolated lipomas can be addressed without unnecessary mesh placement.

Ever since inguinal hernia repair with placement of a mesh was introduced, postoperative attention shifted towards chronic postoperative inguinal pain (CPIP). To lower the incidence of postoperative pain risk factors need to be eliminated. Many of these risk factor have already been addressed. With the introduction of minimally invasive surgery the inguinal nerves were avoided by placing the mesh in the pre-peritoneal space. Additionally, it became known that completing the surgical learning curve and a proper selection of the weight of the mesh reduces postoperative pain.19,20 Another commonly known risk factor of CPIP is "young age". ${ }^{16}$ Where the most recent international inguinal hernia guidelines are based on outdated research and predominantly on data of Lichtenstein repairs, Chapter 7 clearly points out that in a centre of expertise young adults (18-30 years) do not experience more CPIP after TEP hernia repair compared to older adults ( $\geq 31$ years). Patients over 18 years of age that undergo an inguinal hernia repair without placement of a mesh are more prone to develop a recurrence than patients that undergo mesh repair. Regarding efficiency, postoperative pain rates are not increased in this age group and therefore a non-mesh repair does not outweigh the risk of developing a recurrence considering the amount of visits to the GP and/or outpatient clinic, additional imaging, absence from work and re-operations. We recommend performing TEP inguinal hernia repair with placement of a mesh in patients of 18-30 years of age.

\section{Conclusions}

The following conclusions may be drawn from the studies presented in this thesis:

- Single-visit endoscopic TEP inguinal hernia repair is cost-effective compared to regular TEP procedures from both a hospital and a societal perspective, of which the reduction in societal costs is the most contributory. (Chapter 2)

- Ultrasound diagnostics in patients with clinically apparent inguinal hernias is redundantly requested in primary care (Chapter 3 )

- The probability of wrongly attributing groin complaints to an incidental inguinal hernia, diagnosed on ultrasound, is considerable. (Chapter 4)

- Reducing recurrence rates can be achieved by mesh fixation in bilateral, large and direct defects and by thoroughly searching for, and subsequently removing, 
preperitoneal lipomas. (Chapter 5)

- Ultrasound does not need to be incorporated in the diagnostic workup of an apparent recurrent inguinal hernia. (Chapter 6)

- Young adults (18-30 years) do not experience more CPIP after TEP hernia repair compared to older adults ( $\geq 31$ years). (Chapter 7 )

- In the majority of European countries, administration of antibiotic prophylaxis in open inguinal hernia repair can be considered overtreatment (Chapter 8)

\section{Future perspectives:}

Despite the increased quality of inguinal hernia surgery over the past few decades, a demand for improving efficiency remains. Although we have taken some initial steps towards improving the efficiency of inguinal hernia repair, possibilities will always remain to further improve current and future medical efficiency. Considering the high-volume population of inguinal hernia patients efficient improvement, no matter how small, can yield considerable savings of time and costs.

The Dutch healthcare system is organized to keep patients in primary care whenever possible and minimize referrals to a specialist. This way targeted and efficient hospital-care is achieved with reduction of healthcare expenditure. In this type of healthcare system, often referred to as a 'gatekeeper system', the responsibility to decide whether a patient needs hospital care, a diagnostic test or can be treated within a primary care setting lies with the GPs. ${ }^{21}$ Since access to secondary care is reliant on referrals, an adequate PE and diagnosis is important. Although the Dutch College of General Practitioners (NHG) has produced over 100 evidence-based guidelines covering almost $80 \%$ of conditions presented in primary care, currently no guideline with instructions for diagnosis and subsequent treatment of inguinal hernias exists. Since an inguinal hernia is one of the most common reasons a primary care patient may need referral for surgical repair, the absence of such a guideline is a shortcoming in the working relationship between GPs and surgeons. Establishing such a guideline would improve the collaboration of GPs and surgical specialists. Future studies on the current topic are therefore suggested in order to determine whether a primary care inguinal hernia guideline together with proper education of the PE, would reduce referrals to secondary care.

Since groin pain can have a wide variety of possible causes, we do not know whether a clinically occult hernia, diagnosed upon additional imaging, causes the pain. Hence, it is unknown whether patients with occult inguinal hernias benefit from surgical repair. In line with the adagio "do not harm", it would thus be very interesting to know whether a watchful waiting policy with physiotherapy is a suitable alternative. Currently, a randomized multicentre study is being carried out that assesses the influence of a watchful waiting approach compared to surgical intervention by TEP repair for patients with symptomatic clinically occult inguinal hernias. ${ }^{22}$ Findings of this "EFFECT-trial" will provide insight into the effect on pain, quality of 
life and cost-effectiveness up to one year after treatment.

Since simplifying care and avoiding unnecessary hospital visits has a substantial effect on both hospital and societal costs, it will continue to be of great interest for hospitals, governments and societies. For inguinal hernia repair, the first steps have been taken with the single visit pathway. It would be beneficial to further promote this pathway. In the Dutch Hernia Centre alone, based on the matching process of the cost-analysis, at least double the patients could have undergone single-visit surgery. In order to increase the number of single visit patients, it is essential to increase awareness among both patients, referring GPs and insurance companies. Furthermore, it would be appealing to extrapolate the single visit pathway to other frequently performed surgical procedures. Future studies may even determine the feasibility of replacing the physical POS by an electronic POS for all types of operations in all relatively healthy patients (ASA I/II). By doing so a further minimization of hospital and societal costs would be expected.

In the context of increasing efficiency by avoiding waste of time and energy, follow-up after inguinal hernia repair in the Dutch Hernia Centre has experienced a major transformation over the past ten years. Where previous follow-up consisted of a standardised hospital visit including physical examination, current follow-up is performed by means of telephone consultation at 1 day and 6 weeks after surgery. Although this represents progress in the field of medical efficiency, it probably remains a waste of energy. It is possible that follow-up could only be performed upon indication. Furthermore, it could be expected that patients who are well-informed about the uncomplicated postoperative course, will consult the hospital themselves in case of abnormal complaints. However, if we stop standardized follow-up, we lose our ability to register data. Besides assessing potential postoperative complaints, followup is also performed to assess quality objectives for internal quality control and for (inter) national benchmarking. Furthermore, follow-up is of value in research settings. Future research must focus on finding a method of follow-up that is simple and cost-effective, with an efficient gathering of data for quality control, benchmarking and research, and that assures a minimal burden for both patients and surgeons. It would be interesting to see, whether digital followup or data registration through a smartphone application could be a feasible and efficient alternative for the current telephonic follow-up.

\section{REFERENCES}

1. Institute of Medicine. Crossing the Quality Chasm: A New Health System for the 21st Century. Washington, DC: National Academy Press; 2001.

2. Expert Group on Health System Performance Assessment (HSPA) TOOLS AND METHODOLOGIES TO ASSESS THE EFFICIENCY OF HEALTH CARE SERVICES IN EUROPE An overview of current approaches and opportunities for improvement. Publications Office of the European Union, 2019 `

3. Brownlee S, Chalkidou K, Doust J et al. (2017) Evidence for overuse of medical services around the world. Lancet. 2017 July 08; 390(10090): 156-168. 
4. Cylus J, Papanicolas I, Smith PC. Health system efficiency: How to make measurement matter for policy and management. London: World Health Organization - European Observatory on Health Systems and Policies; 2016.

5. European Observatory on Health Systems and Policies (2016) Health system efficiency How to make measurement matter for policy and management Health Policy Series, No. 46 ISBN13: 9789289050418

6. Kashani S, Muhtaseb M, Robinson F (2005) Analysis of 'onestop' referral system for ophthalmic minor operations by GPs. Br J Gen Pract 55(510):53-54

7. van der Geer S, Frunt M, Romero HL, Dellaert NP, JansenVullers MH, Demeyere TB, Neumann HA, Krekels GA (2012) One-stop-shop treatment for basal cell carcinoma, part of a new disease management strategy. J Eur Acad Dermatol Venereol 26(9):1154-1157

8. Bismil M, Bismil Q, Harding D, Harris P, Lamyman E, Sansby L (2012) Transition to total one-stop wideawake hand surgery service-audit: a retrospective review. J R Soc Med Short Rep 3:23

9. Stephenson BM, Callander C, Sage M, Vellacott KD (1993) Feasibility of 'day case' laparoscopic cholecystectomy. Ann R Coll Surg Engl 75(4):249-251

10. Jutte EH, Cense HA, Dur AH, Hunfeld MA, Cramer B, Breederveld RS (2010) A pilot study for one-stop endoscopic total extraperitoneal inguinal hernia repair. Surg Endosc 24(11): 2730-2734

11. Voorbrood CE, Burgmans JP, Clevers GJ, Davids PH, Verleisdonk EJ, Schouten N, van Dalen T (2015) One-stop endoscopic hernia surgery: efficient and satisfactory. Hernia 19(3):395-400

12. Chassin MR, Galvin RW. The urgent need to improve health care quality. Institute of Medicine National Roundtable on Health Care Quality. JAMA. 1998 Sep 16;280(11):1000-1005.

13. Brownlee S, Chalkidou K, Doust J et al. (2017) Evidence for overuse of medical services around the world. Lancet. 2017 July 08; 390(10090): 156-168.

14. Chung L, Norrie J, O'Dwyer PJ (2011) Long-term follow-up of patients with a painless inguinal hernia from a randomized clinical trial. Br J Surg 98(4):596-599.

15. Ventola CL (2015) The antibiotic resistance crisis. Part 1: causes and threats. Pharmcol Ther 40:277-283

16. The Herniasurge Group (2018) International guidelines for groin hernia management. Hernia 22:1-165.

17. Moynihan R, Henry D, Moons KG. Using evidence to combat overdiagnosis and overtreatment: evaluating treatments, tests, and disease definitions in the time of too much. PLoS Med 2014;11:e1001655

18. Ministerie van Volksgezondheid, Welzijn en Sport (2018) Outcome-based Healthcare 2018-2022.

19. Schouten N, Simmermacher RK, van Dalen T et al (2013) Is there an end of the "learning curve" of endoscopic totally extraperitoneal (TEP) hernia repair? Surg Endosc 27(3):789-794 
20. Burgmans JPJ, Voorbrood EH, Simmermacher RKJ, Schouten N, Smakman N, Clevers GJ, et al. Longterm results of a randomized double-blinded prospective trial of lightweight (Ultrapro) versus a heavyweight mesh (Prolene) in laparoscopic total extraperitoneal inguinal hernia repair (TULP-trial) Ann Surg. 2016;263:862-866.

21. Whitaker P (2016) GPs are much more than gatekeepers BMJ. 2016 May 18;353:i2751. doi: 10.1136/bmj. i2751.

22. MM Roos, EJMM Verleisdonk, FBM Sanders, AW Hoes, RK Stellato, GWJ Frederix, GJ Clevers, RKJ Simmermacher, JPJ Burgmans, EFFECT study group (2018) Effectiveness of endoscopic totally extraperitoneal (TEP) hernia 29 correction for clinically occult inguinal hernia (EFFECT): study protocol for a randomized controlled trial. Trials 2018;19:322 


\title{
Liesbreukchirurgie
}

\section{ORDE IN VERSCHEIDENHEID}

\author{
Coen V. van Hessen \\ Ine P.J. Burgmans
}

Egbert Jan M.M. Verleisdonk

Diakonessenhuis, afd. Heelkunde, Utrecht

Coen V. van Hessen, medisch student en onderzoeker

Dr. J.P.J. Burgman, chirurg

Dr. E.J.M.M. Verleisdonk, chirurg 


\title{
Liesbreukchirurgie: orde in verscheidenheid
}

\author{
Coen V. van Hessen, J.P.J. (Ine) Burgmans en Egbert Jan M.M. Verleisdonk
}

\begin{abstract}
Met circa 30.000 ingrepen per jaar is de correctie van een liesbreuk een van de meest uitgevoerde operaties in Nederland. Je zou dus denken dat inmiddels wereldwijd dezelfde techniek wordt gebruikt. Toch zijn er verschillende procedures, afhankelijk van de leeftijd en voorgeschiedenis van de patiënt, het type liesbreuk en de expertise van de chirurg.
\end{abstract}

Toen eind 19e eeuw de pathofysiologie van een liesbreuk duidelijk werd, legde Bassini de basis voor de moderne liesbreukchirurgie: het verstevigen van de achterwand van het lieskanaal na het reponeren van de breukzak met inhoud.

Hoewel de huidige operatieve behandeling nog steeds volgens dit principe werkt, bestaan hiervoor inmiddels veel verschillende technieken. Sommige zijn genoemd naar de 'uitvinder' van een dergelijke operatie (Shouldice, Lichtenstein en Stoppa) en andere worden aangegeven met een afkorting (TEP, TAPP, TIPP en TREPP).

In dit overzichtsartikel beschrijven wij de chirurgische technieken die nu het gangbaarst zijn in Nederland en scheppen wij orde in het woud aan eigennamen en afkortingen.

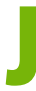
aarlijks worden in Nederland circa 30.000 liesbreuken chirurgisch gecorrigeerd, wat deze ingreep een van de vaakst uitgevoerde operaties maakt. ${ }^{1} \mathrm{Bij}$ een zo vaak uitgevoerde operatie zou je denken dat inmiddels wereldwijd dezelfde techniek wordt gebruikt. Niets is echter minder waar.

Pas eind 19e eeuw werd de pathofysiologie van een liesbreuk duidelijk. In die tijd legde de Italiaanse chirurg Edoardo Bassini de basis voor de moderne liesbreukchirurgie: het verstevigen van de achterwand van het lieskanaal na het reponeren van de breukzak met inhoud. ${ }^{2}$ Het primair sluiten van het buikwanddefect resulteerde echter in een hoog percentage recidieven en in pijn. Door het gebruik van een kunststof matje werd een spanningsloze sluiting en versteviging van het lieskanaal mogelijk en daalde het aantal recidieven naar minder dan $5 \%$. Chronische pijn, die met name wordt veroorzaakt door beschadiging van de sensibele zenuwen in de lies, bleef echter een groot probleem.

In de jaren 90 van de vorige eeuw werden laparoscopische alternatieven ontwikkeld, waarbij het matje in de preperitoneale ruimte wordt geplaatst om zenuwschade te beperken. ${ }^{3}$ De leercurve van deze technieken bleek echter lang en de kosten waren aanvankelijk hoog. ${ }^{4}$ Open technieken waarbij de mat preperitoneaal wordt geplaatst, worden de laatste jaren verder onderzocht.

Hoewel de huidige operatieve behandeling nog steeds als doel heeft de achterwand te verstevigen, bestaan hiervoor inmiddels veel verschillende technieken. Sommige zijn genoemd naar de 'uitvinder' van een dergelijke ope- 


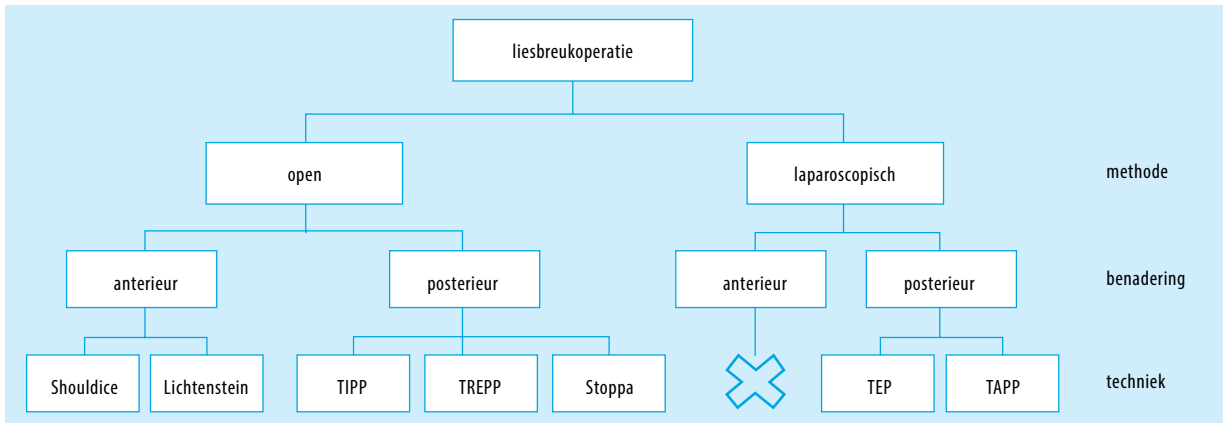

FIGUUR 1 Stroomschema van de indeling van chirurgische technieken voor een liesbreukoperatie.

$\mathrm{TIPP}=$ transinguinaal preperitoneaal; TREPP $=$ transrectusschede-preperitoneaal; TEP $=$ totaal extraperitoneaal; TAPP $=$ transabdominaal preperitoneaal.

ratie (Shouldice, Lichtenstein en Stoppa) en andere worden aangegeven met een afkorting (TEP, TAPP, TIPP en TREPP).

In dit overzichtsartikel beschrijven wij de chirurgische technieken die nu het gangbaarst zijn in Nederland en scheppen wij orde in het woud aan eigennamen en afkortingen. Ook bespreken we kort de voor- en nadelen van de verschillende technieken.

\section{DE TECHNIEK}

De essentie van een liesbreukcorrectie is het benaderen van het buikwanddefect en het reponeren van de buikinhoud, waarna resectie of repositie van de breukzak plaatsvindt. Hierna dient de 'slappe' achterwand te worden hersteld of verstevigd.

De verschillende technieken zijn in te delen in een open en een laparoscopische methode, die op hun beurt weer kunnen worden ingedeeld op basis van de benadering van het buikwanddefect (figuur 1). Bij een anterieure of voorste benadering wordt het defect aan de voorzijde van de buikwand verholpen met hechtingen (Shouldicetechniek) of een matje (Lichtenstein-techniek). Bij een posterieure benadering wordt de liesbreuk opgeheven met een matje aan de binnenzijde van de buikwand in de preperitoneale ruimte (TIPP-, TREPP-, Stoppa-, TEP- en TAPP-techniek).

\section{OPEN ANTERIEURE BENADERING}

\section{SHOULDICE-TECHNIEK}

Bij de liesbreukcorrectie volgens Shouldice wordt een incisie gemaakt in de lies (figuur 2), waarbij de breuk wordt gereponeerd en het defect in de buikwand wordt ongedaan gemaakt door meerdere lagen van de buikwand aan elkaar te hechten (figuur 3a). ${ }^{5}$ Er wordt geen matje geplaatst.

De laatste jaren werd deze techniek bijna niet meer gebruikt, maar door onrust in de media over het gebruik van kunststof matjes en mogelijke complicaties vragen

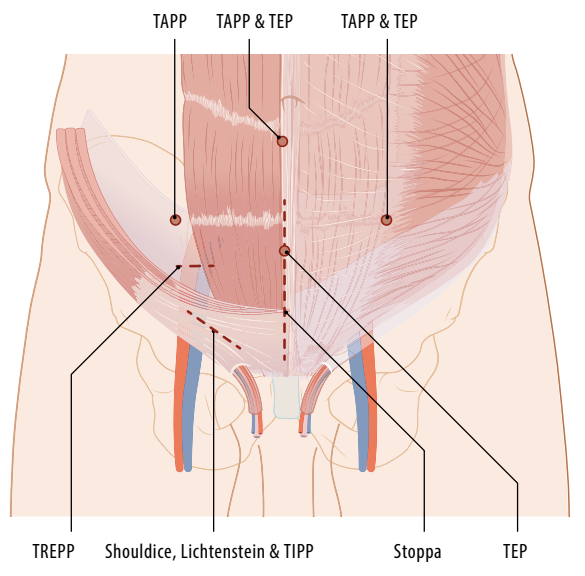

FIGUUR 2 Plaats van de buikwandincisie of -incisies bij chirurgische technieken voor een liesbreukoperatie.

TIPP $=$ transinguinaal preperitoneaal; TREPP $=$ transrectusschede-

preperitoneaal; TEP = totaal extraperitoneaal; TAPP = transabdominaal preperitoneaal. 

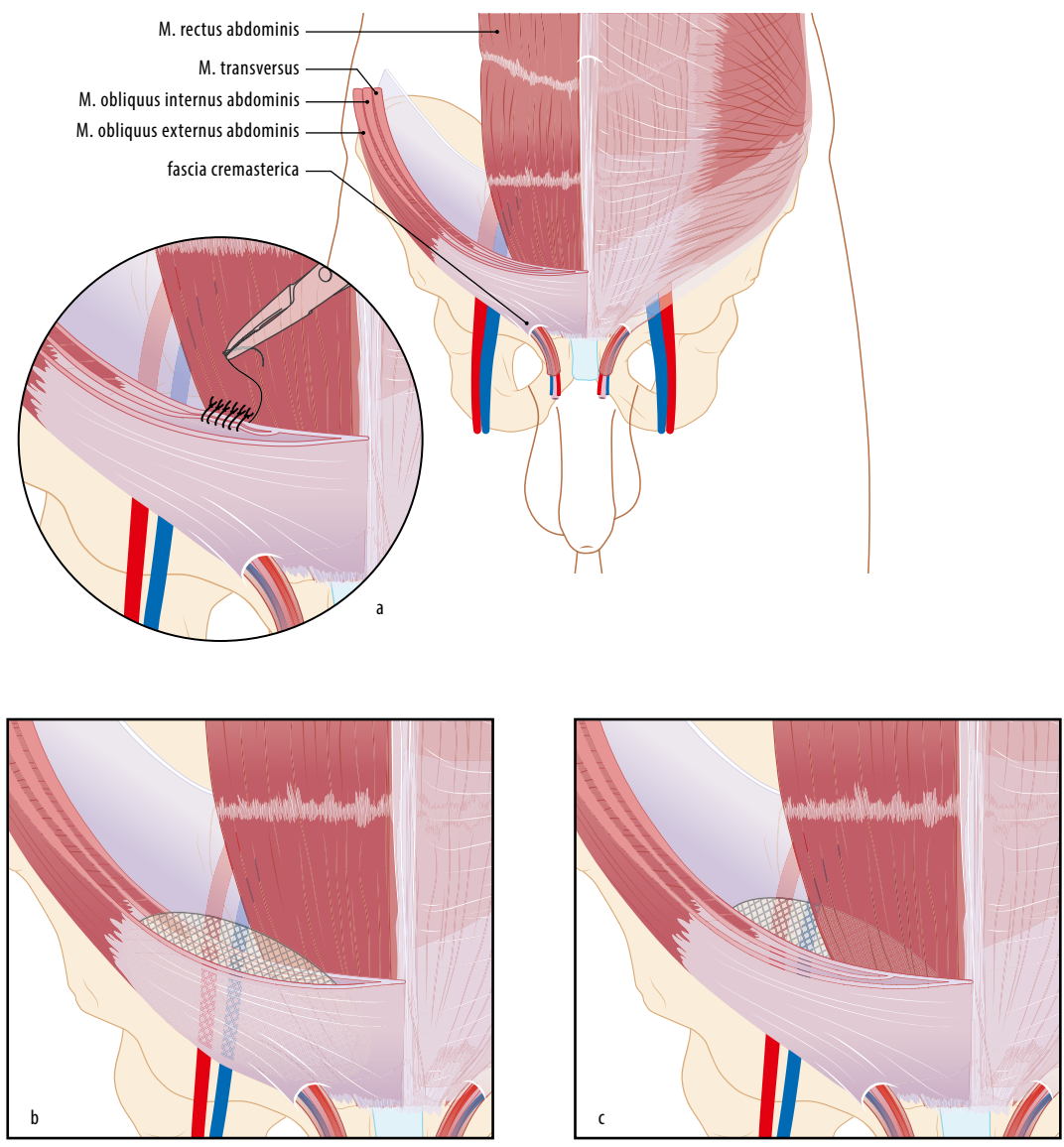

FIGUUR 3 (a) Doorlopende hechtingen bij de Shouldice-techniek. (b) Plaatsing van een matje bij de Lichtenstein-techniek. (c) Plaatsing van een preperitoneaal matje.

sommige patiënten nu om liesbreukcorrectie zonder mat. Het risico op een recidief is hoog bij deze techniek.

\section{LICHTENSTEIN-TECHNIEK}

De liesbreukcorrectie volgens Lichtenstein is nog steeds de meest gebruikte open techniek. ${ }^{6}$ Ook bij deze procedure wordt de incisie in de lies gemaakt (zie figuur 2). De breuk wordt gecorrigeerd door de plaatsing van een matje, dat wordt ingehecht tussen twee spierlagen van de buikwand: de M. obliquus internus abdominis en het peesblad van de M. obliquus externus abdominis (figuur $3 \mathrm{~b}$ ).
Aangezien tussen deze spierlagen ook de sensibele zenuwen van de liesregio liggen, is het percentage patiënten met chronische pijn na deze operatie relatief hoog.

\section{OPEN POSTERIEURE BENADERING}

\section{TRANSINGUINAAL PREPERITONEAAL}

De transinguinale preperitoneale (TIPP) liesbreukcorrectie is een variatie op de open liesbreukcorrectie volgens Lichtenstein en werd in 2006 voor het eerst beschreven. ${ }^{7} \mathrm{Bij}$ deze procedure is de incisie en de benadering 
TABEL Voor- en nadelen van chirurgische technieken voor een liesbreukoperatie*

\begin{tabular}{|c|c|c|c|c|c|c|c|}
\hline kenmerk & Shouldice & Lichtenstein & TIPP† & TREPP† & Stoppa† & TEP & TAPP \\
\hline \multicolumn{8}{|l|}{ voordeel } \\
\hline lokale verdoving & + & + & + & - & - & - & - \\
\hline peroperatief overzichtł & - & - & + & + & + & ++ & ++ \\
\hline vlug postoperatief herstel & - & - & + & + & -- & ++ & ++ \\
\hline snelle leercurve & $+/-$ & + & - & - & - & -- & - \\
\hline \multicolumn{8}{|l|}{ nadeel } \\
\hline recidiefrisico & ++ & - & - & - & - & - & - \\
\hline postoperatieve pijn & ++ & ++ & - & - & - & - & - \\
\hline wondinfectie & + & + & $+/-$ & $+/-$ & + & - & - \\
\hline
\end{tabular}

aanvankelijk hetzelfde als bij de Lichtenstein-techniek (zie figuur 2), maar passeert de chirurg alle lagen van de buikwand in het liesgebied om het matje in de preperitoneale ruimte te plaatsen (figuur 3c).

Gezien de preperitoneale positie van de mat is het percentage patiënten dat postoperatieve pijn heeft relatief laag. Een nadeel van deze techniek is dat er in de liesregio zowel anterieur als posterieur littekenweefsel ontstaat, wat het kiezen van een techniek bij het ontstaan van een recidief moeilijk maakt. De uitkomsten van deze techniek zijn nog onvoldoende onderzocht.

\section{TRANSRECTUSSCHEDE-PREPERITONEAAL}

De transrectusschede-preperitoneale (TREPP) procedure is de recentst ontwikkelde open liesbreukcorrectie in Nederland en is geïntroduceerd vanuit het ziekenhuis St Jansdal in Harderwijk. ${ }^{8}$ Ook bij deze techniek wordt het matje preperitoneaal geplaatst (zie figuur 3c). De incisie wordt hierbij echter niet in de lies geplaatst, maar enkele centimeters onder de navel, ter hoogte van de laterale rectusschede (zie figuur 2). Hierbij blijft de liesregio anatomisch intact en liggen de sensibele zenuwen van de liesregio niet in het operatiegebied.

Ook deze techniek is in Nederland vrij nieuw. De resultaten zijn te beperkt om aanbevelingen te kunnen doen.

\section{STOPPA-TECHNIEK}

De liesbreukcorrectie volgens Stoppa wordt sinds haar intreden in 1973 nog steeds toegepast, ${ }^{9}$ maar is een grote operatie die met name geïndiceerd is bij forse (bilaterale) scrotale breuken, om een grote toegang te verkrijgen tot de preperitoneale ruimte. Via een verticale incisie in de onderbuik wordt de preperitoneale ruimte benaderd (zie figuur 2), waar het matje wordt geplaatst (zie figuur $3 \mathrm{c}$ ). Bij de Stoppa-techniek opereert men niet in het lieskanaal.

Het herstel na de operatie is relatief lang.

\section{LAPAROSCOPISCHE POSTERIEURE BENADERING}

\section{TOTAAL EXTRAPERITONEAAL}

De totale extraperitoneale (TEP) liesbreukcorrectie is een operatietechniek die steeds vaker wordt uitgevoerd vanwege het snelle herstel en het lage percentage patiënten dat nadien pijn heeft. ${ }^{10-12}$ De TEP-procedure wordt gezien als de laparoscopische versie van de Stoppa-techniek. De benadering is via 3 kleine incisies (zie figuur 2). Tijdens de operatie blijft de chirurg buiten het peritoneum en wordt het matje preperitoneaal geplaatst (zie figuur 3c).

Een nadeel is dat de TEP-techniek een technisch ingewikkelde ingreep is met een lange leercurve. Ruime expertise is daarom nodig.

\section{TRANSABDOMINAAL PREPERITONEAAL}

Bij de transabdominale preperitoneale (TAPP) liesbreukcorrectie wordt de breuk eveneens via 3 kleine incisies benaderd (zie figuur 2) ${ }^{13,14}$ Hierbij opereert men echter intraperitoneaal. Om het matje in de preperitoneale ruimte te kunnen plaatsen (zie figuur $3 \mathrm{c}$ ), wordt het peritoneum daarom eerst geopend om daarna weer te worden gesloten met bijvoorbeeld hechtingen of nietjes ('staples').

Net als bij de TEPP-is het herstel na de TAPP-procedure 
snel en het risico op chronische pijn laag, maar kent de techniek een relatief lange leercurve.

\section{TOEPASSING}

In de tabel zijn de voor- en nadelen van elke besproken techniek samengevat. Wat de beste techniek is voor de behandeling van een liesbreuk is afhankelijk van de leeftijd van de patiënt, comorbiditeit, eventuele eerdere operaties, het type breuk, de wens van de patiënt en de chirurgische expertise. De 'World guidelines for groin hernia management' is opgesteld door een internationale groep chirurgen, de HerniaSurge Group, en biedt houvast bij het maken van een keuze voor een bepaalde techniek; deze richtlijn zal in het eerste kwartaal van 2018 worden gepubliceerd. De situatie in Nederland is echter in een aantal opzichten niet vergelijkbaar met die in andere landen en continenten en daarom wordt momenteel een richtlijn geschreven die toepasbaar is voor liesbreukchirurgie in Nederland.

\section{KINDEREN}

Bij kinderen tot 18 jaar met een liesbreuk wordt afgezien van de plaatsing van een matje. De oorzaak van de liesbreuk bij kinderen ligt namelijk niet in een zwakte van de achterwand, maar in het onvolledig sluiten van de processus vaginalis, dit is de embryologische uitstulping van het buikvlies waarlangs bij jongens de testis afdaalt naar het scrotum. De behandeling bestaat uit resectie van de breukzak.

\section{OUDEREN MET COMORBIDITEIT}

Bij oudere patiënten met veel comorbiditeit kan volledige narcose gevaarlijk zijn of is spinale anesthesie bezwaarlijk. Soms wordt dan gekozen voor lokale verdoving. De Lichtenstein-techniek is veilig en geschikt om onder lokale verdoving uit te voeren en kan bij deze patiëntengroep de voorkeur hebben.

\section{VROUWEN EN DE FEMORALE BREUK}

Een femorale breuk (ook wel 'dijbeenbreuk' of 'damesliesbreuk' genoemd) is een breuk waarbij de buikinhoud uitstulpt in het dijbeenkanaal (canalis femoralis). De femorale breuk is lastig te diagnosticeren en wordt makkelijk gemist bij een anterieure benadering volgens Lichtenstein.

De femorale breuk komt 4 keer vaker voor bij vrouwen dan bij mannen. Bij vrouwen met een liesbreuk wordt daarom aangeraden om standaard een laparoscopische
LEERPUNTEN

- De correctie van een liesbreuk is een van de meest uitgevoerde operaties in Nederland.

- Er bestaan veel verschillende chirurgische technieken voor een liesbreukcorrectie, maar het principe is hetzelfde: versteviging van de achterwand na het reponeren van de breukzak met inhoud.

- Wat de beste techniek is voor de behandeling van een liesbreuk is sterk afhankelijk van de leeftijd en comorbiditeit van de patiënt, eventuele eerdere operaties, het type breuk, de wens van de patiënt, het land en de chirurgische expertise.

- Begin 2018 zal een internationale groep chirurgen, de HerniaSurge Group, de 'World guidelines for groin hernia management' uitbrengen; een Nederlandse richtlijn voor liesbreukchirurgie wordt momenteel geschreven.

liesbreukbehandeling te verrichten, om een eventuele femorale breuk niet te missen.

\section{MANNEN}

Bij gezonde mannen met een eerste enkelzijdige liesbreuk wordt een laparoscopische TEP- of TAPP-procedure aangeraden vanwege het lage percentage patiënten met postoperatieve pijn en het vlotte herstel. De resultaten zijn echter sterk afhankelijk van de expertise van de chirurg. Als de betrokken chirurg onvoldoende laparoscopische ervaring heeft, heeft de Lichtenstein-techniek de voorkeur.

\section{RECIDIEF}

Het risico op een recidief van een liesbreuk varieert in Nederland van 1-11\% en is afhankelijk van het type breuk, de techniek en de chirurgische expertise. De beste techniek voor het opheffen van een recidief is afhankelijk van de techniek die bij de eerste operatie werd uitgevoerd: een recidief na een anterieure benadering vraagt om een posterieure benadering en omgekeerd, vanwege littekenvorming die bij de eerdere operatie is ontstaan. 


\section{LITERATUUR}

1 StatLine. Centraal Bureau voor Statistiek. http://statline.cbs.nl/Statweb, geraadpleegd op 1 mei 2017.

2 Bassini E. Ueber die Behandlung des Leistenbruches. Langenbecks Arch Klin Chir. 1890;40:429-76.

3 Read RC. Herniology: past, present, and future. Hernia. 2009;13:577-80.

4 Schouten N, Simmermacher RK, van Dalen T, et al. Is there an end of the learning curve of endoscopic totally extraperitoneal (TEP) hernia repair? Surg Endosc. 2013;27:789-94.

5 Shouldice EE. Surgical treatment of hernia. Ont Med Rev. 1945;2:43.

6 Lichtenstein IL, Shore JM. Simplified repair of femoral and recurrent inguinal hernias by a plug technic. Am J Surg. 1974;128:439-44.

7 Pélissier EP. Pelissier EP Inguinal hernia: preperitoneal placement of a memory-ring patch by anterior approach. Preliminary experience.

Hernia. 2006;10:248-52.

8 Prins MW, Voropai DA. C.J.H.M van Laarhoven, W.L. Akkersdijk. De transrectusschede-preperitoneale procedure. Een veilige, effectieve en goedkope chirurgische benadering voor een liesbreuk? Ned Tijdschr Geneeskd. 2013;156:A6048.
9 Stoppa R, et al. Procedé original de plastie des hernies de l'aine: l'interposition sans fixation d'une prothèse en tulle de Dacron par voie médiane sous-péritonéale. Rev Med Picardie. 1972;1:46-8.

1o McKernan J. Laparoscopic repair of inguinal hernias using a totally extraperitoneal prosthetic approach. Sur Endosc. 1993;7:26-8.

11 Stoker DL, Spiegelhalter DJ, Singh R, Wellwood JM. Laparoscopic versus open inguinal hernia repair: randomised prospective trial. Lancet. 1994;343:1243-5

12 Burgmans JP, Voorbrood CE, Simmermacher RK, et al. Long-term results of a randomized double-blinded prospective trial of a lightweight (ultrapro) versus a heavyweight mesh (prolene) in laparoscopic total extraperitoneal inguinal hernia repair (TULP-trial). Ann Surg. 2016;263:862-6.

13 Hawasli A. Laparoscopic inguinal herniorrhaphy: classification and 1 year experience. J Laparoend Surg. 1992;2:137-43.

14 Corbitt JD Jr. Transabdominal preperitoneal herniorrhaphy. Surg Laparosc Endosc. 1993;3:328-32. 


\section{Nederlandse Samenvatting}

Een liesbreuk is een veel voorkomende aandoening en de liesbreukoperatie is de meest uitgevoerde chirurgische ingreep wereldwijd. Jaarlijks worden in Nederland circa 30.000 liesbreuken geopereerd. Ongeveer een derde van de mannen ontwikkeld in zijn leven een liesbreuk.

De diagnose liesbreuk wordt in de meeste gevallen gesteld door middel van anamnese en lichamelijk onderzoek. Aanvullend onderzoek is zelden nodig. Beeldvorming is alleen van toegevoegde waarde indien er sprake is van klinische twijfel. In dit geval heeft echografie de voorkeur.

Niet alleen de diagnose is vaak eenvoudig, ook de essentie van een liesbreukcorrectie is simpel. Er is sprake van een te wijde opening van het lieskanaal of een 'slappe' achterwand. Een liesbreuk hersteld nooit spontaan en moet operatief hersteld worden door de 'slappe achterwand' te verstevigen. Dit is alleen nodig bij liesbreuken die klachten geven. Het is verantwoord om bij asymptomatische of minimaal symptomatische liesbreuken een afwachtend beleid te voeren, aangezien de kans op inklemming erg klein is.

In de afgelopen decennia is de kwaliteit van de liesbreukoperatie enorm verbeterd. De kans op een recidief is met het standaard gebruik van een kunststof matje gedaald tot minder dan $5 \%$ en ook het percentage postoperatieve pijn is afgenomen, mede door de introductie van laparoscopie. Hoewel er een grote verscheidenheid is aan chirurgische technieken, patiënten liesbreuk-gerelateerde karakteristieken en lokale of nationale voorzieningen, worden de laparoscopische technieken (TEP en TAPP) wereldwijd steeds vaker toegepast. Mits er voldoende expertise voorhanden is, resulteren deze operaties in minder chronische pijn en een sneller herstel.

De technische verbetering van de liesbreukoperatie lijkt een plafond te hebben bereikt en de percentages postoperatieve pijn en recidieven zijn acceptabel laag. We zien de laatste jaren een toenemende trend naar het verbeteren van doelmatigheid in de liesbreukzorg, waarbij de focus steeds meer verschuift naar zorg op maat en kosteneffectiviteit. Gezien de stijgende gezondheidszorgkosten staat doelmatigheid van de zorg al een aantal jaren hoog op de politieke agenda. Verscheidene studies schatten in dat meer dan $20 \%$ van de wereldwijde uitgaven aan gezondheidszorg kan worden gezien als verspilling. Hierbij gaat het om medische handelingen die ineffectief zijn of duurder zijn dan de (even effectieve) alternatieven. Artsen worden dan ook niet alleen verantwoordelijk gehouden voor het verbeteren van de kwaliteit van de zorg, maar zijn ook verplicht om de efficiëntie van de zorg te verhogen en verspilling tegen te gaan. Dit kan onder andere worden bereikt door zorg te vereenvoudigen, de administratie te verminderen, fouten en complicaties te reduceren en overbodige of kwalitatief ondermaatse handelingen te vermijden (zoals onnodige medische beeldvorming of operaties bij patiënten 
Voor het verbeteren van de doelmatigheid van liesbreukzorg, moeten eerst alle stappen binnen het zorgpad in kaart worden gebracht. Dat begint bij de afspraak met de huisarts, waarbij achtereenvolgens de chirurgische intake, de preoperatieve screening, de operatie en de postoperatieve zorg moeten worden geëvalueerd. Alle stappen dienen kritisch bekeken te worden. Om de efficiëntie te verhogen zal het zorgpad moeten worden versneld, ziekenhuisbezoeken worden geminimaliseerd en gestreefd worden naar een vlot herstel. Bovendien zal het voorkomen van overmatig gebruik van medische beeldvorming of antibiotica, het kritisch beoordelen van het nut van een operatie en het verder verminderen van complicaties (met de bijbehorende kosten) allemaal een belangrijke rol spelen bij doelmatige liesbreukzorg.

De onderzoeksresultaten van dit proefschrift zijn verkregen in een groot expertisecentrum voor liesbreukchirurgie waarbij de TEP (Totale Extraperitoneale Plastiek) de eerste behandelkeuze is. In dit centrum worden jaarlijks meer dan 1200 operaties uitgevoerd door 5 gespecialiseerde liesbreukchirurgen. De afdelingen chirurgie, radiologie en anesthesie werken nauw samen voor optimale zorg van liesbreuken.

Een belangrijke stap in doelmatige liesbreukzorg is het versnellen en het vereenvoudigen van het zorgpad van de patiënt met klachten van een liesbreuk. De meeste liesbreuk patiënten zijn gezonde, werkende mannen. Een goed voorbeeld van het vereenvoudigen van het zorgpad is de 'single-visit' TEP liesbreukcorrectie., Deze methode, waarbij de preoperatieve beoordeling en de operatie van een patiënt op één dag plaatsvinden, blijkt al langer een geschikte behandeling met een hoog opkomstpercentage (99\%), een hoog percentage patiënten dat daadwerkelijk dezelfde dag geopereerd wordt (96\%) en een hoge patiënttevredenheid (gemiddeld score van 9 op een schaal van 0 tot 10). Intuïtief is een 'single-visit' TEP liesbreukcorrectie ook kostenbesparend, maar dit is tot op heden niet goed geanalyseerd. In Hoofdstuk 2 wordt een kostenanalyse beschreven die de 'single-visit' en de reguliere TEP liesbreukcorrectie vergelijkt vanuit het perspectief van zowel ziekenhuiskosten als van maatschappelijke kosten. De resultaten laten zien dat de 'single-visit' procedure voordeliger blijkt dan de reguliere TEP procedure. Bij een werkende gezonde populatie leidt de 'single-visit' procedure tot een kostenreductie van $20 \%$, waarvan het merendeel wordt bepaald door een vermindering van de maatschappelijke kosten. De kostenreductie in het ziekenhuis is relatief klein en werd voornamelijk bepaald door het verschil in preoperatieve screening (POS) van een reguliere procedure (in het ziekenhuis) en de 'single-visit' procedure (telefonische/elektronisch POS). De maatschappelijke kosten van de 'single-visit' procedure bleken echter met een kwart gedaald te zijn ten opzichte van de reguliere procedure. Deze reductie kon worden verklaard door het afgenomen aantal ziekenhuisbezoeken, gepaard gaande met minder reiskosten en een lager aantal gemiste werkdagen. Aangezien zowel werkgevers als werknemers het meeste baat zullen hebben bij het aanpassen van het zorgpad van patiënten met een liesbreuk ín het ziekenhuis, is het voor ziekenhuizen zeker de moeite waard om diverse zorgpaden in het ziekenhuis opnieuw te evalueren. Het implementeren van een 'single-visit' zorgpad voor andere veel voorkomende electieve chirurgische procedures (bijv. cholecystectomie, colorectale procedures en/of spataderen) en de daarbij komende maatschappelijke kostenverlaging, zou kunnen zorgen voor een daling van de gezondheidszorgkosten. 
Een tweede belangrijke pijler voor doelmatige zorg, is het vermijden van overbodige of kwalitatief ondermaatse medische handelingen. Binnen de liesbreukzorg dient hierbij met name gefocust te worden op onnodige medische beeldvorming. Het is bekend dat de overgrote meerderheid van liesbreuken een primair klinische diagnose is op basis van medische voorgeschiedenis en lichamelijk onderzoek. In de praktijk wordt echter veelvuldig op aanvraag van de huisarts een echografie van de lies verricht voordat patiënten verwezen worden naar de chirurgische polikliniek. Het is de vraag of deze aanvullende diagnostiek altijd nodig is en of deze doorslaggevend is voor het wel of niet verwijzen naar de tweede lijn. Het verminderen van onnodige diagnostiek in de eerste lijn (en de daarmee gepaard gaande kosten en tijdverspilling) is een belangrijke stap in het verhogen van de efficiëntie. Hoofdstuk 3 bevat een kritische evaluatie van het aantal aangevraagde diagnostische echografieën voorafgaand aan de verwijzing naar de chirurg. De resultaten van deze studie laten zien dat huisartsen bij ongeveer een kwart van de patiënten een echografie aanvragen voordat patiënten worden verwezen. Bij ongeveer $80 \%$ van deze patiënten werd bij lichamelijk onderzoek door een chirurg een duidelijke liesbreuk gevonden en had de echografie waarschijnlijk achterwege kunnen blijven. Het is niet vreemd dat huisartsen sneller geneigd zijn een echografie aan te vragen in het geval van liesklachten. Er kan niet verwacht worden dat het lichamelijk onderzoek van een huisarts -die slechts enkele keren per jaar een patiënt met een liesbreuk ziet- even goed is als dat van een ervaren chirurg die wekelijks liesbreuken diagnosticeert. Echter, het aanvragen van echografie om een verdenking op een liesbreuk te bevestigen is overbodig. Tevens is het aanvragen van echografieën uit angst de diagnose te missen of uit angst voor een beklemde liesbreuk onnodig, zeker gezien de lage kans op beklemming $(<3 \%$ per jaar). Adequate training van huisartsen in het uitvoeren van het lichamelijk onderzoek en bewustwording van de overtollig aangevraagde echografieën kan een stap betekenen in de zoektocht naar doelmatigheid. Met het oog op efficiëntie valt daarbij te betwisten of het aanvragen van een echografie van de lies hoort bij de eerste- of tweedelijns zorg.

Omdat een echografie van de lies vaak wordt aangevraagd bij klinische twijfel (bijvoorbeeld bij aspecifieke pijn in de lies zonder zichtbare zwelling), komt het regelmatig voor dat een liesbreuk wordt vastgesteld op een echografie zonder dat er klinische kenmerken aanwezig zijn van een liesbreuk. In deze gevallen is het de vraag of een dergelijke liesbreuk op de echografie daadwerkelijk de oorzaak is van de klachten. Het gevaar van al deze gemaakte echografieën is dan ook overdiagnostiek, wat kan leiden tot onnodige operaties van liesbreuken die geen klinische consequenties hebben (of zullen ontwikkelen indien er niet behandeld wordt). Alle studies die tot nu toe zijn gepubliceerd over echografie van de lies betreffen evidente liesbreuken of symptomatische klinisch occulte liesbreuken. Onderzoek naar de prevalentie van liesbreuken als toevalsbevinding op echografie is echter nooit gepubliceerd. In Hoofdstuk 4 hebben we de prevalentie van een subklinische liesbreuk bepaald bij gezonde mannen in de werkende leeftijd zonder liesklachten, om inzicht te krijgen in de kans dat liesklachten ten onrechte worden toegeschreven aan een liesbreuk. De resultaten van dit onderzoek laten zien dat bij $16 \%$ van deze mannen er een liesbreuk geconstateerd wordt bij echografie van de lies. Deze hoge prevalentie en het grootschalige gebruik van echografieën, geeft dus een aanzienlijk risico op het toevallig diagnosticeren van een liesbreuk die ten onrechte wordt beschouwd als de oorzaak van liespijn. In dit scenario is de potentiële schade groter dan de voordelen van een operatie. In het geval van liesklachten zonder duidelijke 
klinische kenmerken van een liesbreuk, moeten zowel huisartsen als chirurgen zich bewust zijn van deze prevalentie en dus van het risico op overdiagnostiek. Het is belangrijk de brede differentiaal diagnose van liespijn te kennen en eerst andere oorzaken uit te sluiten.

Hoewel de recidiefpercentages laag zijn sinds de introductie van het matje, blijven recidieven na een TEP liesbreukoperatie een klinisch probleem. De kosten van recidieven zijn vaak hoog door pijnstillers, meerdere bezoeken aan de huisarts en/of polikliniek, aanvullende beeldvorming, verzuim en her-operaties. Het begrijpen van de etiologie van een recidief en het identificeren van de mogelijke oorzaken kan helpen bij het voorkomen van recidieven, het verhogen van de efficiëntie en de reductie van kosten. Gezien de doorgaans lage recidiefpercentages vormt dit een uitdaging. $\mathrm{Er}$ is een groot aantal patiënten nodig om gedegen onderzoek naar recidieven te doen. Echter, gezien een liesbreukoperatie een van de meest uitgevoerde chirurgische ingrepen is, kunnen zelfs kleine verbeteringen leiden tot aanzienlijke vooruitgang van de gezondheidszorg. Om meer inzicht te verkrijgen in de oorzaak van het ontwikkelen van een recidief in een hypothetisch optimale situatie, werd een 11-jaars analyse verricht van alle patiënten die een her-operaties ondergingen vanwege een verdenking op een recidief na een eerdere liesbreukcorrectie middels een TEP uitgevoerd in een expertisecentrum voor TEP. De resultaten van deze studie staan beschreven in Hoofdstuk 5. Aangezien alle chirurgen uitgebreide ervaring hadden, mocht worden aangenomen dat geen van de recidieven gerelateerd waren aan een onvoltooide leercurve. Driekwart van alle recidieven betrof een directe (mediale) liesbreuk na een primaire directe liesbreuk. Bij bijna een vijfde bleek het recidief te gaan om een lipoom dat een 'echt' recidief nabootste. Deze lipomen zijn mogelijk ontstaan na de primaire operatie. Er is echter ook een reële kans dat deze lipomen al aanwezig waren, maar werden gemist gedurende de primaire operatie. De resultaten van deze studie geven tevens aan dat bij patiënten bij wie een primair groot defect werd gezien, de liesbreuken recidiveerde als hun initiële type, met name wanneer het matje niet was gefixeerd. Daarbij komt dat patiënten die een bilaterale TEP procedure ondergingen meer kans hadden op een recidief. Daarom adviseren wij, in het licht van doelmatigheid, de mat te fixeren bij grote directe en bilaterale liesbreuken. Tevens is het belangrijk om de volledige achterwand en het lieskanaal grondig te inspecteren om lipomen adequaat te verwijderen. Het advies is een vermeend recidief te behandelen met een anterieure benadering. Met behulp van deze benadering worden 'echte' recidieven behandeld volgens de internationale richtlijnen (met een anterieure correctie met matje) en kunnen geïsoleerde lipomen worden behandeld zonder onnodige mat-plaatsing.

In het merendeel van de gevallen zijn de klinische kenmerken van een recidief liesbreuk gelijk aan die van een primaire liesbreuk. Het diagnosticeren van een recidief liesbreuk na een open liesbreukoperatie kan echter lastig zijn. In de internationale richtlijnen wordt lichamelijk onderzoek dan ook in combinatie met echografie van de lies aanbevolen als meest geschikt om de diagnose te stellen. Het is echter onbekend of dit ook geldt voor recidieven na een laparoscopische liesbreukoperatie. Toch zien we dat zowel huisartsen als chirurgen veelvuldig een echografie aanvragen alvorens een patiënt geopereerd wordt aan een recidief. Hoofdstuk 6 beschrijft dat bijna twee derde van de patiënten die een her-operatie ondergingen op verdenking van een recidief liesbreuk, eerst een echografie van de lies hadden ondergaan, waarvan het merendeel door de chirurg werd aangevraagd. Aangezien een positief lichamelijk 
onderzoek van een chirurg in 97\% van de gevallen overeenkwam met de peroperatieve bevindingen, was het echografisch onderzoek in retrospectie niet nodig geweest. In het algemeen geldt dat aanvullende beeldvorming niet noodzakelijkerwijs geïncorporeerd hoeft te worden bij het diagnosticeren van een recidief liesbreuk. Alleen in het geval van klinische twijfel kan echografie van toegevoegde waarde zijn. Ook bij recidief liesbreuken geldt dat het mogelijk efficiënter zou kunnen zijn om patiënten rechtstreeks door te verwijzen naar de chirurgische polikliniek in plaats van echografie aan te vragen vanuit de eerstelijns zorg.

Naast recidieven blijft ook postoperatieve pijn of chronische postoperatieve pijn (CPIP) een belangrijke complicatie van een liesbreuk operatie. Hoewel het percentage relatief laag is, zou een verdere daling van dit percentage een positieve weerslag hebben op de efficiëntie van liesbreukzorg door een vermindering van het aantal poliklinische bezoeken, pijnstillers, ziekteverzuim en her-operaties. Om de incidentie van postoperatieve pijn te verminderen, moeten de risicofactoren worden weggenomen. Veel van deze risicofactoren zijn reeds geïdentificeerd en aanpassingen van technieken geïmplementeerd. Met de introductie van minimaal invasieve chirurgie werd het mogelijk het matje in de pre-peritoneale ruimte te plaatsen waarbij de kans op beschadiging van de lies-zenuwen geminimaliseerd werd. Ook het voltooien van de chirurgische leercurve en de juiste keuze van het gewicht van de mat zijn belangrijk bij het verminderen van de kans op postoperatieve pijn. Een andere risicofactor van CPIP is het hebben van een "jonge leeftijd". Om postoperatieve pijn te verminderen, suggereren verschillende auteurs dat een open breukzakresectie zonder matplaatsing of plastiek de voorkeur heeft bij jongeren. Een duidelijke definitie van 'jonge leeftijd' bestaat er echter niet. Bovendien is de risicofactor voornamelijk gebaseerd op onderzoeken naar open liesbreukcorrecties. In Hoofdstuk 7 hebben we uitgezocht of jongvolwassenen (18-30 jaar) meer kans hebben op het ontwikkelen van CPIP na een TEP liesbreukcorrectie in vergelijking met volwassenen ouder dan 30 jaar. De resultaten van dit onderzoek laten zien dat (in een expertisecentrum) jongvolwassenen na een TEP liesbreukcorrectie niet meer chronische pijnklachten ontwikkelen dan oudere volwassenen. Omdat we weten dat volwassen patiënten een hogere kans hebben op een recidief liesbreuk na een operatie zonder matplaatsing, raden wij aan om ook bij de jongere patiënt tussen 18 en 30 jaar een matje te plaatsen met een TEP. Met het oog op doelmatigheid zal dit het risico op recidieven verkleinen en daarmee resulteren in minder bezoeken aan de huisarts en/of polikliniek, aanvullende beeldvorming, gemiste werkdagen en her-operaties.

Hoewel een laparoscopische techniek, mits in ervaren handen, de voorkeur heeft in het merendeel van de liesbreuken, worden open liesbreukcorrecties (met name de Lichtenstein techniek) nog steeds op grote schaal uitgevoerd. Een laparoscopische operatie wordt beschouwd als een steriele chirurgische techniek met een lage kans op postoperatieve wondinfecties, waarbij er geen meerwaarde is voor het gebruik van antibiotica profylaxe. Er blijft echter discussie over de vraag of antibiotische profylaxe gebruikt moet worden in open liesbreuk chirurgie. Het niet toedienen van antibiotische profylaxe bij een gecontamineerde chirurgische procedure kan leiden tot wondinfecties en zorgt derhalve voor meer gezondheidsrisico en extra kosten. Het onnodig toedienen van antibiotische profylaxe bij steriele chirurgische procedures kan echter worden beschouwd als overbehandeling. Hoofdstuk 8 beschrijft een systematisch literatuuronderzoek naar alle beschikbare data uit gerandomiseerde trials waarin antibiotische 
profylaxe wordt vergeleken met een placebo (of geen profylaxe) bij open liesbreukoperaties. De resultaten tonen aan dat toediening van antibioticaprofylaxe voor electieve open liesbreukoperaties niet universeel moet worden aanbevolen. Alleen in een omgeving met een hoog infectierisico kan antibiotische profylaxe oppervlakkige wondinfecties verminderen. In gebieden met een laag infectierisico heeft antibioticaprofylaxe echter weinig of geen effect op de vermindering van deze oppervlakkige wondinfecties. Ongeacht de omgeving en het infectierisico kon de preventie van diepe wondinfecties door antibiotische profylaxe niet worden bevestigd of ontkracht in vergelijking met een placebo (of geen behandeling). Daarom mag in de meeste Europese landen toediening van antibioticaprofylaxe bij open liesbreukcorrecties in het algemeen als overbehandeling worden beschouwd. 
List of publications

Acknowledgements

Curriculum Vitae 


\section{List of publications}

van Hessen CV, Burgmans JPJ, Verleisdonk EJMM. Inguinal hernia repair: bringing order to variety.

Ned Tijdschr Geneeskd 2018;162:D2216

van Hessen CV, Roos MM, Sanders FBM, Verleisdonk EJMM, Clevers GJ, Davids PHP, Burgmans JPJ

Recurrence after totally extraperitoneal (TEP) inguinal hernia repair; the role of physical examination and ultrasound. Hernia 2020 Feb;24(1):153-157

van Hessen CV, Roos MM, Frederix GWJ, Verleisdonk EJMM, Clevers GJ, Davids PHP, Burgmans JPJ. One-stop routing for surgical interventions; a cost-analysis of endoscopic groin repair Surg Endosc 2020 May;34(5):1968-1977

Roos MM, van Hessen CV, Verleisdonk Egbert Jan MM, Clevers Geert Jan, Davids Paul HP, Voorbrood Charlotte EH, Simmermacher Rogier KJ, Burgmans Josephina PJ. An 11-year analysis of reoperated groins after endoscopic totally extraperitoneal (TEP) inguinal hernia repair in a high volume hernia center. Hernia 2019 Aug;23(4):655-662.

Bakker WJ, van Hessen CV, Verleisdonk EJMM, Clevers GJ, Davids PHP, Schouten N, Burgmans JPJ. Is young age a risk factor for chronic postoperative inguinal pain after endoscopic totally extraperitoneal (TEP) repair? Hernia 2019 Dec;23(6):1053-1059.

Orelio CC, van Hessen CV, Sanchez-Manuel FJ, Aufenacker TJ, Scholten RJ. Antibiotic prophylaxis for prevention of postoperative wound infection in adults undergoing open elective inguinal or femoral hernia repair. Cochrane Database Syst Rev. $2020 \mathrm{Apr}$ 21;4(4):CDO03769.

Bakker WJ, van Hessen CV, Clevers GJ, Verleisdonk EJMM, Burgmans JPJ. Value and patient appreciation of follow-up after endoscopic totally extraperitoneal (TEP) inguinal hernia repair. Hernia. 2020 Oct;24(5):1033-1040.

van Hessen CV*, Roos MM*, Sanders FBM, Verleisdonk EJMM, de Vries A, Clevers GJ, Burgmans JPJ. Groin ultrasound for diagnosing inguinal hernia in primary care. Submitted

van Hessen CV, Bakker WJ Sanders FBM, Verleisdonk EJMM, Clevers GJ, Davids PHP, Avci M, Burgmans JPJ. Incidental inguinal hernia in men. Submitted

Rinkel WD, Bakker WJ, van Hessen CV, Burgmans JPJ. Non-Descendend Testicle Presnting as an Inguinal Hernia. Surgical Case Reports, ISSN 2613-5965 


\section{Acknowledgements / Dankwoord}

Geachte Prof. Dr. I.H.M. Borel Rinkes, beste Inne. Hoewel ik van tevoren wist dat onze samenwerking zich met name zou centreren rondom het einde van mijn promotietraject, was ik blij verrast dat onze wegen zich al maanden hiervoor kruisten bij het chirurgencabaret. Het enthousiasme waarmee $u$ tijdens het chirurgencabaret de rol van pianist en bandleider op $u$ nam, werkte aanstekelijk. Ditzelfde enthousiasme merkte ik in het contact voorafgaande aan mijn promotie. Door helder en direct commentaar, gecombineerd met de nodige dosis humor, lukte het om mijn proefschrift efficiënt af te ronden. Bedankt dat u mijn promotor wilde zijn.

Dr. J.P.J. Burgmans, geachte copromotor, beste Ine. Wat een feest was het om onder jouw directe begeleiding dit traject aan te gaan. Je eeuwige betrokkenheid en enthousiasme ondanks je drukke bestaan, zorgde voor een bijzonder prettige sfeer. Al ben ik er achter gekomen dat wetenschappelijk onderzoek niet mijn roeping is, heeft het aan jouw enthousiasme en kennis zeker niet gelegen. Dat een promotietraject bovenal heel gezellig kan zijn is wel gebleken tijdens onze avonturen tijdens de buitenlandse congressen. Rikken in San Francisco, lunchen onder de Eiffeltoren in Las Vegas en een middagje sightseeing in Hamburg; slechts een kleine greep uit de bijzondere momenten die in 2 jaar de revue passeerden. Wellicht was het zelfs iets te gezellig, want in het inspirerende 'la Baraque' in Zevenhuizen bleek toch wel dat ik een tandje bij moest zetten;). Bedankt voor alles, ik hoop in de toekomst contact te blijven houden. Alle belangrijke stappen in mijn muziekcarrière zal ik in ieder geval met je blijven delen, dat beloof ik!

Dr. E.J.M.M. Verleisdonk, geachte copromotor, beste Egbert Jan, ome Eppie. Wie had 15 jaar geleden, toen ik sputterend van het snot achter je aan fietste in de hoop eerder op de top van de Splugenpass te zijn, ooit kunnen bevroeden dat ik je nu officieel in mijn proefschrift zou mogen bedanken voor alles. Ik in ieder geval niet. Toch moet ik zeggen dat ik in mijn korte carrière al een hoop aan je te danken heb gehad. Ik benijd je om je energie en daadkracht en heb je in je werk niet anders ervaren dan dat ik je in privé al kende. lets dat ik heel mooi vind om te merken. Dank voor alles.

Geachte leden van de beoordelingscommissie, bestaande uit prof. dr. F.G. Huisman, prof. dr. F. Berrevoet, prof. dr. R.M. Pijnappel, Dr. R.K.J. Simmermacher en prof. dr. R. van Hillegersberg. Bedankt voor het beoordelen van mijn proefschrift en het plaatsnemen in de commissie.

Chirurgen dr. G.J. Clevers, dr. P.H.P. Davids en dr. M. Avci, beste Geert Jan, Paul en Murat. Bedankt voor jullie bijdrage als coauteurs aan dit proefschrift. Zonder jullie operaties en data viel er voor mij weinig op te schrijven.

Dr. M.M. Roos, beste Marleen. Promoveren kun je leren, maar als je de kneepjes van het vak van jou leert, wordt het wel een heel gespreid bedje. Waar jij destijds een hoop zelf uit hebt moeten zoeken, kon ik altijd een beroep op je doen. Zelfs toen je al lang en breed je geluk als huisarts aan het najagen was, was er altijd tijd om even te sparren. Je stond niet alleen aan de wieg van mijn onderzoek, maar ook aan die van mijn muzikale carrière. Jouw voorliefde voor hiphop en R\&B heeft mijn repertoire enorm verrijkt en daar ben ik je wellicht nog wel het meest dankbaar voor! 
Drs. W.J. Bakker, beste Wouter. Na meer dan een jaar samen gezeten te hebben was het een behoorlijke domper toen jij de kliniek in ging. Als "Ine's mannen" oftewel Jut en Jul, waren we geruime tijd onafscheidelijk. Toen jij in je witte jas opeens ècht hard ging werken en ik alleen in ons onderzoekshok overbleef, was de lol er dan ook snel vanaf. Gelukkig waren er toen nog de congressen en borrels. En wat hebben we daar een paar mooie momenten meegepakt. Veel meer nog dan voor je bijdrage aan mijn boekje, wil ik je bedanken voor de gezelligheid. Ik verheug me op het feit dat we binnenkort weer collega's worden. Met een brede lach en met een goede vriend rijker kan ik dit liesbreukavontuur afsluiten. Nog even en jij zal volgen.

Drs. F.B.M. Sanders, beste Floris, ik wil je bedanken voor je betrokkenheid en waardevolle bijdrage vanuit de radiologie bij de verschillende studies, alsmede voor de kritische revisie van mijn stukken.

Alle overige auteurs wil ik bedanken voor hun bijdrage aan de hoofdstukken van dit proefschrift.

Overige chirurgen en alle arts-assistenten Heelkunde van het Diakonessenhuis, bedankt voor de goede sfeer in de kliniek tijdens mijn eerste klinische baan. Ik zal het nog gaan missen na 3 jaar bij jullie te hebben rondgelopen.

Dr. C.C. Orelio, beste Claudia. Bedankt voor je hulp en adviezen bij de verschillende projecten en bedankt voor de mogelijkheid die je me gaf om mee te doen aan het Cochrane review.

Levien en Spook, ik vind het fijn dat jullie naast me staan op 26 november. Met Covid als grootste opponent is het nog maar de vraag hoe de dag zal verlopen. Als jullie maar weten dat dat feest er ooit nog komt. Daar zorgen we met z'n drieën voor.

Lieve familie en vrienden. Dank jullie wel voor alle support, inspiratie, afleiding, grappen, geduld en bovenal dat jullie niet te veel hebben gefocust op mijn promotie :) Ik was er waarschijnlijk heel onrustig van geworden.

Lieve Sanne, toen je me na ons etentje bij Ine vroeg wat dan eigenlijk een TEP was waar we het steeds over hadden, besefte ik dat ik wellicht wat doorgeschoten was in het scheiden van werk en privé. Toch had ik jouw rol tijdens mijn promotie niet beter kunnen wensen. Het gaat om de kleine dingen. Dat je er altijd was en nog steeds altijd voor me bent, is me het allermeeste waard. Ik ben je heel erg dankbaar voor je geduld, humor, oprechte interesse, zorgzaamheid en de ruimte die je me geeft als ik die soms nodig heb. Dit hoofdstuk is nu af. Met veel enthousiasme gaan we op naar de volgende stap in ons leven. 


\section{Curriculum Vitae}

Coenraad Victor van Hessen was born on the 1st of November 1991 in Utrecht, the Netherlands. $\mathrm{He}$ is the middle one of three children of Maarten van Hessen and Erica Paul. He attended high school at the St. Bonifatiuscollege in Utrecht and graduated in 2009, after which he started his bachelor's degree in Medicine at the University of Amsterdam.

During his study he enjoined acting, singing and going on tour with the cabaret trio Malt with whom he was involved in two major cabaret contests (Groningen Studenten Cabaret Festival and Leids Cabaret Festival). In 2014 he took a year out to serve on the board of the Utrechtsch Studenten Corps.

During the final phase of his master's degree in Medicine he started as a research student at the Liesbreuk Centrum Nederland to lay the foundation for his PhD thesis. After obtaining his master's degree in 2017, he started working as a PhD candidate in the field of inguinal hernia surgery at the Diakonessenhuis Utrecht/Zeist under supervision of dr. J.P.J. Burgmans and dr. E.J.M.M. Verleisdonk, in cooperation with the University Medical Center Utrecht (Promoter Prof. Dr. I.H.M. Borel Rinkes).

Currently Coen works as a surgical resident (not in training) at the Diakonessenhuis Utrecht under supervision of $d r$. Th. Van Dalen. In his spare time he established a second career as producer and DJ, known professionally as 'Saco'. In December Coen will start as a doctor at a nursing home, to gain further clinical experience before applying for a residency in general medical practice. In the future, he hopes to combine working as a general practitioner with producing music. 



\section{Efficiency In \\ Inguinal Hernia Repair}

Coen V. van Hessen 



\section{Efficiency In Inguinal Hernia Repair}

Coen V. van Hessen 